\title{
Total Synthesis of Nuclear Factor of Activated T-Cells-68 (NFAT-68): Sequential Use of Chiral Allenylsilane and Titanium Alkoxide-Mediated Reductive Coupling Bond Construction
}

Bin Cai, Ryan W. Evans, Jie Wu, and James S. Panek*

Department of Chemistry, Metcalf Center for Science and Engineering, Boston University, 590 Commonwealth Avenue, Boston, Massachusetts 02215, United States

\section{Table of Contents}

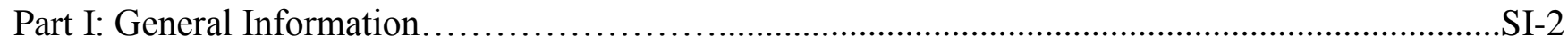

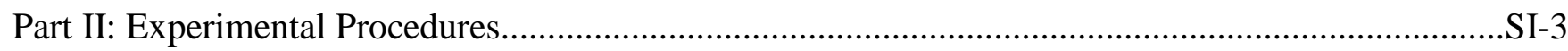

i. Preparation of Chiral Allenylsilanes................................................................................SI-3

ii. Supplementary Table S1: Asymmetric Three-Component Propargylation

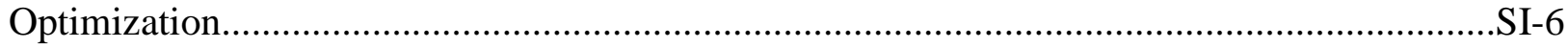

iii. Asymmetric Three-Component Propargylation...............................................................

iv. Supplementary Table S2: Titanocyclopropene Complex Generation Optimization.

v. Supplementary Table S3: Optimization for Alkyne-Alkyne Reductive Coupling between 5b and Methyl Propiolate.

vi. Alkyne-Alkyne Reductive Coupling

vii. Total Synthesis of ent-NFAT-68 and NFAT-68.

a) Total Synthesis of ent-NFAT-68.

b) Total Synthesis of NFAT-68.

viii. NFAT-68 Spectral Data Comparison.

ix. Confirmation of Relative Stereochemistry of ent-8 and Absolute Stereochemistry of 9 and Ent9.

a) Confirmation of Relative Stereochemistry of $\boldsymbol{e n t} \mathbf{- 8}$.

b) Confirmation of Absolute Stereochemistry of 9 and ent-9.

SI-25

Part III: ${ }^{1} \mathrm{H}$ - and ${ }^{13} \mathrm{C}-\mathrm{NMR}$ Spectra. 


\section{Part I: General Information:}

All reactions were carried out in oven or flame-dried glassware under argon atmosphere unless otherwise specified. Triethylamine was distilled over calcium hydride and stored over potassium hydroxide. Dichloromethane, tetrahydrofuran, and toluene were obtained from a dry solvent system (alumina) and used without further drying. Propionitrile was distilled over calcium hydride and stored over $4 \AA$ molecular sieves. Cyclopentylmagnesium chloride was purchased from Sigma-Aldrich, and was titrated by the Watson-Eastham method ${ }^{1}$ using 1,10-phenanthroline and sec-butanol. Aldehydes were freshly distilled before use and stored under nitrogen at $4{ }^{\circ} \mathrm{C}$. Lithium chloride was purchased from Alfa Aesar and vacuum dried at $120{ }^{\circ} \mathrm{C}$ for 12 hours before use. All other reagents were used as supplied. Unless otherwise noted, reactions were magnetically stirred and monitored by thin layer chromatography with Macherey Nagel Polygram $0.20 \mathrm{~mm}$ silica gel $60 \AA$ plates. Flash chromatography was performed on Sorbent Technologies 32-63 $\mu \mathrm{m} 60 \AA$ silica gel. Yields referred to chromatographically and spectroscopically pure compounds, unless otherwise noted. ${ }^{1} \mathrm{H}$ and ${ }^{13} \mathrm{C} \mathrm{NMR}$ spectra were taken in $\mathrm{CDCl}_{3}, \mathrm{C}_{6} \mathrm{D}_{6}$, and $\mathrm{CD}_{3} \mathrm{OD}$ at 400 or $500 \mathrm{MHz}$ (as indicated), respectively. Chemical shifts are reported as major diastereomer and/or regioisomer in parts per million using the solvent internal standard (chloroform, 7.24 and $77.0 \mathrm{ppm}$, benzene, 7.16 and $128.06 \mathrm{ppm}$, and methanol, 3.30 and $47.58 \mathrm{ppm}$ respectively). Data are reported as follows: chemical shift, multiplicity $(\mathrm{s}=$ singlet, $\mathrm{d}=$ doublet, $\mathrm{t}=$ triplet, $\mathrm{q}=$ quartet, $\mathrm{m}=$ multiplet, br $=$ broad), coupling constant, integration. Infrared resonance spectra were recorded on a Nexus 670 FT-IR spectrometer. Optical rotations were recorded on a Rudolph Autopol II digital polarimeter at $589 \mathrm{~nm}$ and reported as follows: $[\alpha]_{\mathrm{D}}^{20}$ (concentration in $\mathrm{g} / 100 \mathrm{~mL}$ solvent and solvent). High resolution mass-spectra were obtained on a Waters Q-TOF Mass Spectrometer at Boston University Chemical Instrumentation Center (CIC).

\footnotetext{
${ }^{1}$ Watson, S. C.; Eastham, J. F. J. Organomet. Chem. 1967, 9, 165
} 


\section{Part II: Experimental Procedures:}

\section{i. Preparation of Chiral Allenylsilanes}

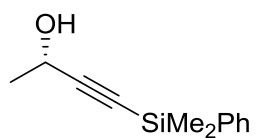

(S)-11

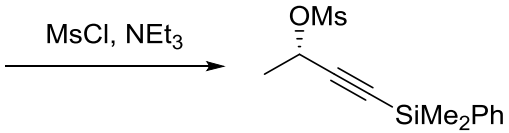

(S)-12

(S)-4-(dimethyl(phenyl)silyl)but-3-yn-2-yl methanesulfonate ((S)-12): A solution of $(S)-\mathbf{1 1}^{2}(39.6 \mathrm{~g}$, $193.7 \mathrm{mmol}, 1$ equiv) and methanesulfonyl chloride $(19.6 \mathrm{~mL}, 253.33 \mathrm{mmol}, 1.3$ equiv) in $774 \mathrm{~mL}$ dry dichloromethane was cooled to $-78{ }^{\circ} \mathrm{C}$. Triethylamine ( $34.3 \mathrm{~mL}, 220.9 \mathrm{mmol}, 1.2$ equiv) was added in dropwise. The resulting mixture was stirred for 12 hours, gradually warming to room temperature. The reaction was quenched with $400 \mathrm{~mL}$ DI water. It was then extract with dichloromethane $(3 \times 300 \mathrm{~mL})$, and the combined organic layers were dried over $\mathrm{MgSO}_{4}$, filtered, and concentrated. Purification over silica gel chromatography (11.4\% EtOAc/Hexanes) afforded product $(S)$-12 as a colorless oil (46.4g, $85 \%$ yield $) .[\alpha]_{\mathrm{D}}^{20}=-8.5\left(c 1.9, \mathrm{CH}_{2} \mathrm{Cl}_{2}\right)$;

${ }^{1} \mathbf{H}$ NMR (500 MHz, $\left.\mathrm{CDCl}_{3}\right): \delta 7.61-7.59(\mathrm{~m}, 2 \mathrm{H}), 7.44-7.38(\mathrm{~m}, 3 \mathrm{H}), 5.31$ (q, $\left.J=6.75 \mathrm{~Hz}, 1 \mathrm{H}\right), 3.04$ (s, $3 \mathrm{H}), 1.67(\mathrm{~d}, J=6.75 \mathrm{~Hz}, 3 \mathrm{H}), 0.46(\mathrm{~s}, 6 \mathrm{H})$;

${ }^{13} \mathrm{C}$ NMR $\left(125 \mathrm{MHz}, \mathrm{CDCl}_{3}\right): \delta 135.61,133.57,129.83,128.06,102.89,91.85,68.44,39.07,22.45,-$ 1.36 ;

IR $\left(\right.$ neat $\left./ \mathrm{cm}^{-1}\right) v_{\max }: 3001,2176,1429,1362,1252,1177,1117,1091,1024,974,921,890,839,815$, $783,734,700,664$;

HRMS (ESI) $m / z$ calcd for $\mathrm{C}_{13} \mathrm{H}_{18} \mathrm{O}_{3} \mathrm{SSi}[\mathrm{M}+\mathrm{Na}]^{+} 305.0644$, found 305.0652 .

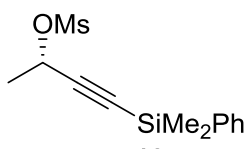

(S)-12

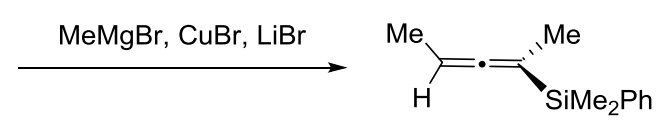

$\left(S_{a}\right)-4$

(S)-dimethyl(penta-2,3-dien-2-yl)(phenyl)silane $\left(S_{a}\right)-4$ : $\mathrm{CuBr}$ was purified before use according to the following procedure: $\mathrm{CuBr}(68.3 \mathrm{~g}, 475.8 \mathrm{mmol})$ was dissolved in $113.3 \mathrm{~mL} \mathrm{HBr}(33 \% \mathrm{w} / \mathrm{w}$ in acetic acid) and stirred until it was fully dissolved. The resulting thick mixture was pour into cold DI water $(2.32 \mathrm{~L})$. Solids formed was filtered, collected, and washed with DI water, $\mathrm{MeOH}$, and $\mathrm{Et}_{2} \mathrm{O}$ sequentially. It was then dried with $\mathrm{LiBr}$ under vacuum at $120^{\circ} \mathrm{C}$ for 12 hours. A solution of $\mathrm{CuBr}(\sim 47.8 \mathrm{~g}, 328.7$ mmol, 2 equiv) and $\mathrm{LiBr}\left(28.5 \mathrm{~g}, 328.7 \mathrm{mmol}, 2\right.$ equiv) in $580 \mathrm{~mL}$ THF was cooled to $0{ }^{\circ} \mathrm{C}$. Methylmagnesium bromide $(110.6 \mathrm{~mL}, 3 \mathrm{M}$ solution in diethyl ether, $328.7 \mathrm{mmol}, 2$ equiv) was added into above solution slowly. The resulting mixture was stirred for 30 min at $0{ }^{\circ} \mathrm{C} .(S)-12(46.4 \mathrm{~g}, 164.3 \mathrm{mmol}$, 1 equiv) in $7 \mathrm{~mL}$ THF was added in. The reaction mixture was stirred for 36 hours, gradually warming to room temperature. The reaction was quenched with $250 \mathrm{~mL}$ DI water. It was then extract with ethyl acetate $(3 \times 300 \mathrm{~mL})$, and the combined organic layers were dried over $\mathrm{MgSO}_{4}$, filtered, and concentrated. Purification over silica gel chromatography $\left(5 \% \mathrm{CH}_{2} \mathrm{Cl}_{2} / \mathrm{Hexanes}\right)$ afforded product $\left(S_{a}\right)-\mathbf{4}$ as a colorless oil $(30 \mathrm{~g}, 90 \%$ yield $)$. $[\alpha]_{\mathrm{D}}^{20}=+2.8\left(c 7.0, \mathrm{CH}_{2} \mathrm{Cl}_{2}\right)$;

\footnotetext{
${ }^{2}$ Brawn, R. A.; Panek, J. S. Org. Lett. 2007, 9, 2689
} 
${ }^{1} \mathbf{H}$ NMR $\left(500 \mathrm{MHz}, \mathrm{CDCl}_{3}\right): \delta 7.55-7.53(\mathrm{~m}, 2 \mathrm{H}), 7.37-7.35(\mathrm{~m}, 3 \mathrm{H}), 4.79-4.73(\mathrm{~m}, 1 \mathrm{H}), 1.65(\mathrm{~d}, J=$ $2.8 \mathrm{~Hz}, 3 \mathrm{H}), 1.62(\mathrm{~d}, J=6.9 \mathrm{~Hz}, 3 \mathrm{H}), 0.35(\mathrm{~s}, 6 \mathrm{H})$;

${ }^{13}$ C NMR $\left(125 \mathrm{MHz}, \mathrm{CDCl}_{3}\right): \delta 207.97,138.30,133.81,128.98,127.73,89.09,79.01,15.99,13.82,-$ $3.15,-3.25$;

IR $\left(\right.$ neat $\left./ \mathrm{cm}^{-1}\right) v_{\max }: 3088,2961,1934,1428,1363,1248,1113,1066,976,872,832,811,778,699$, 660;

LCMS (ES+) $m / z$ calcd for $\mathrm{C}_{13} \mathrm{H}_{18} \mathrm{Si}[\mathrm{M}+\mathrm{Na}]^{+} 225.11$, found 225.12 .

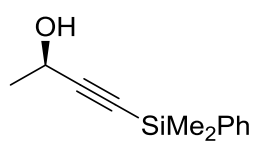

$(R)-11$

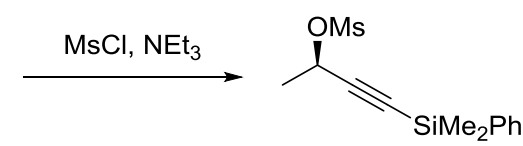

$(R)-12$

(R)-4-(dimethyl(phenyl)silyl)but-3-yn-2-yl methanesulfonate ((R)-12): Made by the same procedure as $(S)-\mathbf{1 2}$, using $(R)-\mathbf{1 1}^{3}$. Purification over silica gel chromatography (11.4\% EtOAc/Hexanes) afforded product $(R)-12$ as a colorless oil $(46.9 \mathrm{~g}, 86 \%$ yield $) .[\alpha]_{\mathrm{D}}^{20}=+8.8\left(c 2.5, \mathrm{CH}_{2} \mathrm{Cl}_{2}\right)$;

${ }^{1} \mathbf{H}$ NMR (500 MHz, $\left.\mathrm{CDCl}_{3}\right)$ : $\delta 7.61-7.59(\mathrm{~m}, 2 \mathrm{H}), 7.44-7.38(\mathrm{~m}, 3 \mathrm{H}), 5.31$ (q, J=6.75 Hz, 1H), 3.04 (s, $3 \mathrm{H}), 1.67(\mathrm{~d}, J=6.75 \mathrm{~Hz}, 3 \mathrm{H}), 0.46(\mathrm{~s}, 6 \mathrm{H})$;

${ }^{13} \mathbf{C}$ NMR $\left(125 \mathrm{MHz}, \mathrm{CDCl}_{3}\right): \delta 135.61,133.57,129.83,128.06,102.89,91.85,68.44,39.07,22.45,-$ 1.36

IR (neat/cm $\left.{ }^{-1}\right) v_{\max }: 3001,2176,1429,1362,1252,1177,1117,1091,1024,974,921,890,839,815$, 783, 734, 700, 664;

HRMS (ESI) $m / z$ calcd for $\mathrm{C}_{13} \mathrm{H}_{18} \mathrm{O}_{3} \mathrm{SSi}[\mathrm{M}+\mathrm{Na}]^{+}$305.0644, found 305.0649 .

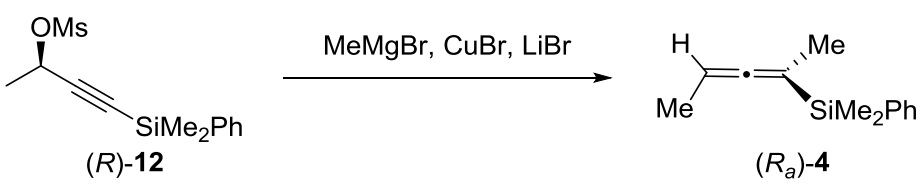

(R)-dimethyl(penta-2,3-dien-2-yl)(phenyl)silane $\left(\boldsymbol{R}_{\boldsymbol{a}}\right)$-4: Made by the same procedure as $\left(S_{a}\right)-\mathbf{4}$, using (R)-12. Purification over silica gel chromatography $\left(5 \% \mathrm{CH}_{2} \mathrm{Cl}_{2} /\right.$ Hexanes $)$ afforded product $\left(R_{a}\right)-4$ as a colorless oil $(46.9 \mathrm{~g}, 86 \%$ yield $) .[\alpha]_{\mathrm{D}}^{20}=-2.8\left(c 7.0, \mathrm{CH}_{2} \mathrm{Cl}_{2}\right)$;

${ }^{1}$ H NMR $\left(500 \mathrm{MHz}, \mathrm{CDCl}_{3}\right): \delta 7.55-7.53(\mathrm{~m}, 2 \mathrm{H}), 7.37-7.35(\mathrm{~m}, 3 \mathrm{H}), 4.79-4.73(\mathrm{~m}, 1 \mathrm{H}), 1.65(\mathrm{~d}, J=$ $2.8 \mathrm{~Hz}, 3 \mathrm{H}), 1.62(\mathrm{~d}, J=6.9 \mathrm{~Hz}, 3 \mathrm{H}), 0.35(\mathrm{~s}, 6 \mathrm{H})$;

${ }^{13}$ C NMR (125 MHz, $\left.\mathrm{CDCl}_{3}\right): \delta 207.97,138.30,133.81,128.98,127.73,89.09,79.01,15.99,13.82,-$ $3.15,-3.25$;

${ }^{3}$ Brawn, R. A.; Panek, J. S. Org. Lett. 2007, 9, 2689 
IR $\left(\right.$ neat $\left./ \mathrm{cm}^{-1}\right) v_{\max }: 3088,2961,1934,1428,1363,1248,1113,1066,976,872,832,811,778,699$, 660 ;

LCMS (ES+) $m / z$ calcd for $\mathrm{C}_{13} \mathrm{H}_{18} \mathrm{Si}[\mathrm{M}+\mathrm{Na}]^{+} 225.11$, found 225.12 .

Ee analysis of $(S)-11$ and $(\boldsymbol{R})-11:(S)-\mathbf{1 1}$, and $(R)-11$ were run on chiral HPLC using a ChiralCel OD-H column, isocratic $1 \%$ ispropanol/hexanes at a flow rate of $1 \mathrm{~mL} / \mathrm{min}$. $(S)-\mathbf{1 1}$ was found to have $96 \%$ ee (however, we believe within system error, the ee would be $>99 \%$ ), and $(R)-\mathbf{1 1}$ was found to have $>99 \%$ ee. Both ee was identical to the reported value. ${ }^{4}$

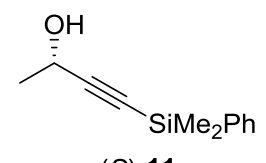

$(S)-11$
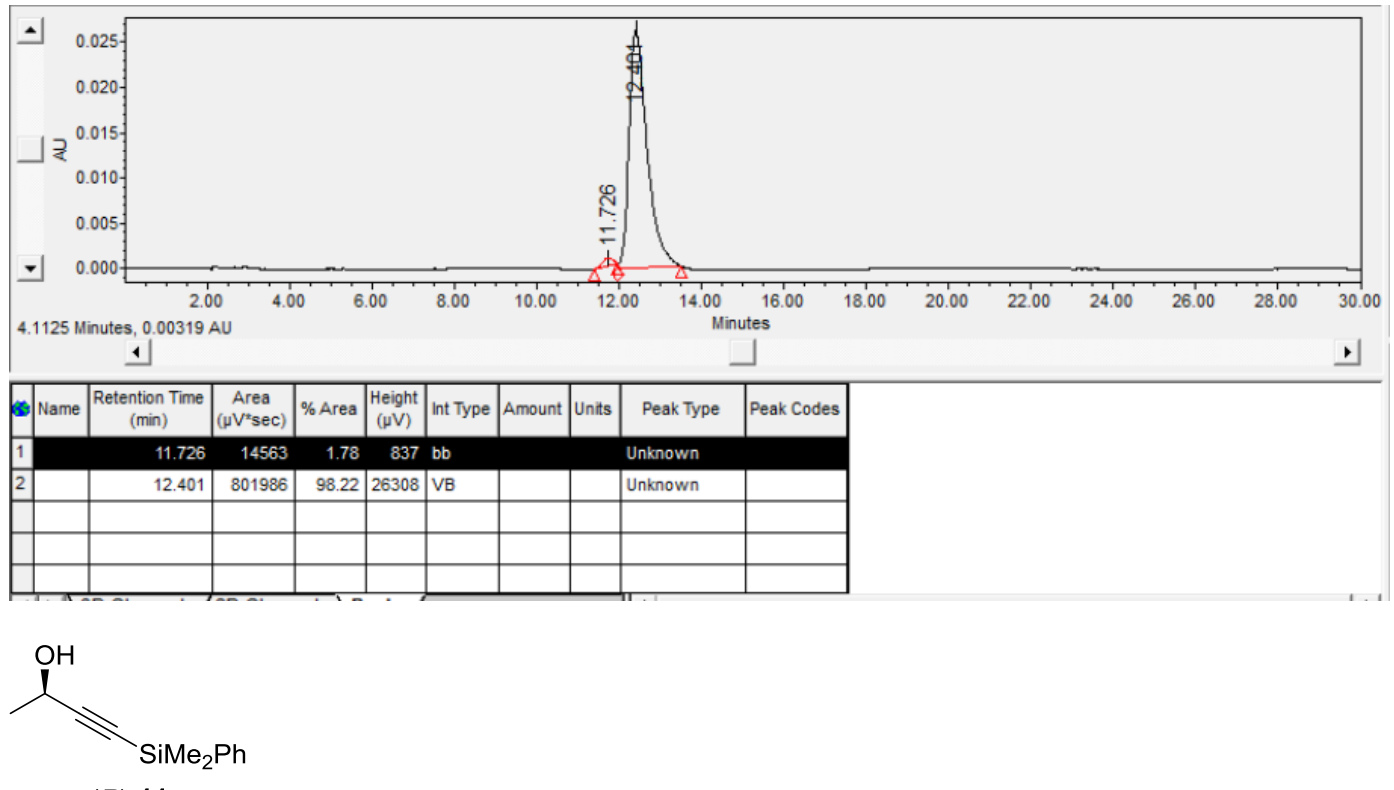

$(R)-11$

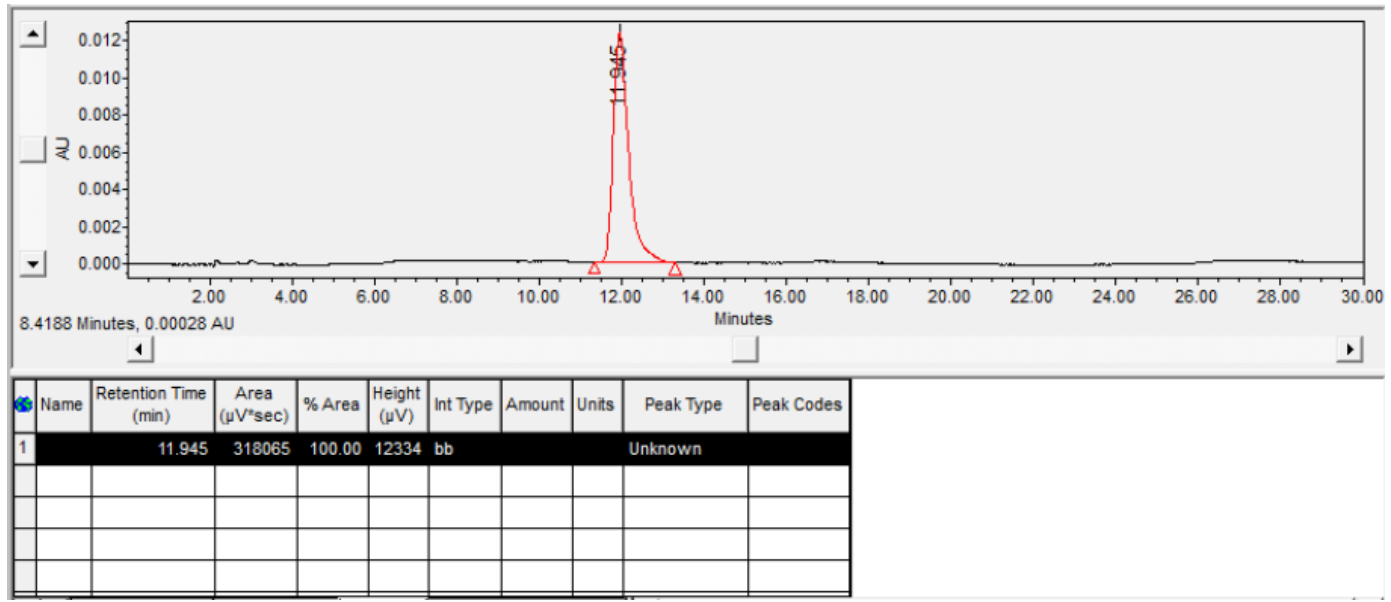

${ }^{4}$ Brawn, R. A.; Panek, J. S. Org. Lett. 2007, 9, 2689 


\section{ii. Supplementary Table S1: Asymmetric Three-Component Propargylation Optimization}

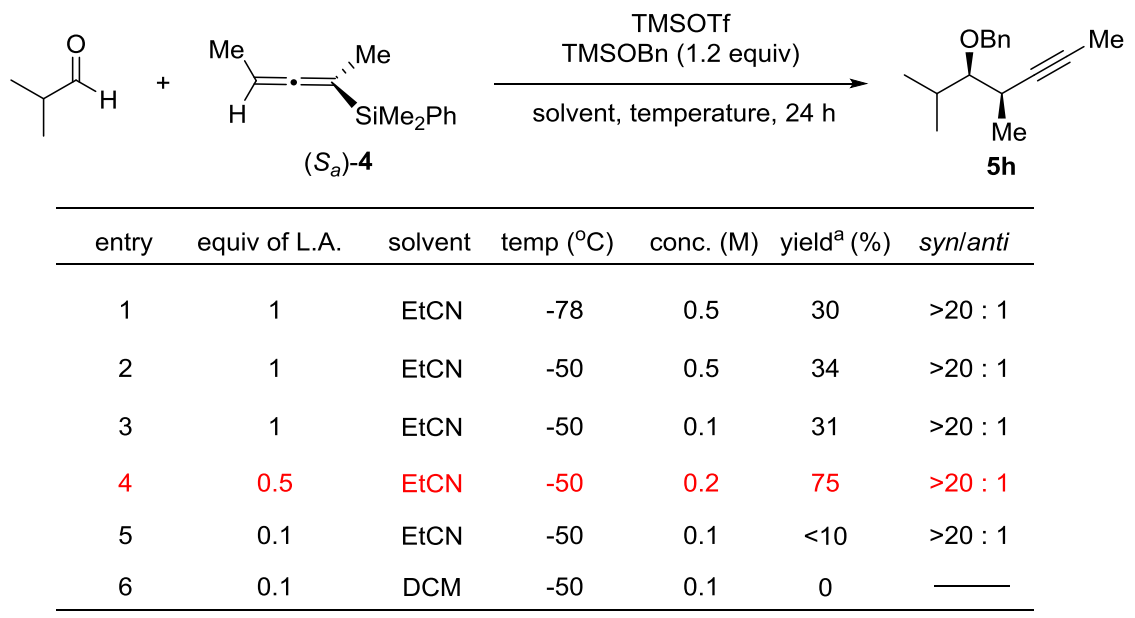

${ }^{a}$ Isolated yield after silica gel chromatography. ${ }^{b}$ Dr based on analysis of crude ${ }^{1} \mathrm{H}$ NMR.

\section{iii. Asymmetric Three-Component Propargylation}

General procedure for asymmetric three-component propargylation between $\left(S_{a}\right) \mathbf{4}, \quad($ benzyloxy)trimethylsilane, and aldehydes: A solution of allenylsilane $(S)-4$ (417 mg, 2.06mmol, 1 equiv), (benzyloxy)trimethylsilane $(0.49 \mathrm{~mL}, 2.47 \mathrm{mmol}, 1.2$ equiv), and aldehyde $(2.47 \mathrm{mmol}, 1.2$ equiv) in 10 $\mathrm{mL}$ propionitrile $(\mathrm{EtCN})$ was cooled to $-78{ }^{\circ} \mathrm{C}$. Trimethylsilyl trifluoromethanesulfonate (TMSOTf) ( $0.19 \mathrm{~mL}, 1.03 \mathrm{mmol}, 0.5$ equiv) was added to above mixture dropwise. The resulting mixture was placed into $-50{ }^{\circ} \mathrm{C}$ chiller and stirred for 24 hours. The reaction was quenched by adding $10 \mathrm{~mL}$ DI water. It was then extract with ethyl acetate $(3 \times 10 \mathrm{~mL})$, and the combined organic layers were dried over $\mathrm{MgSO}_{4}$, filtered, and concentrated. Purification over silica gel chromatography $\left(\mathrm{CH}_{2} \mathrm{Cl}_{2} / \mathrm{Hexanes}\right)$ afforded product $\mathbf{5}$ as an oil.

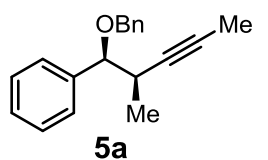

((1S,2R)-1-(benzyloxy)-2-methylpent-3-yn-1-yl)benzene (5a): Prepared by the general procedure using benzaldehyde, alkyne 5a (407 mg, 75\% yield, dr: 3.5:1) was obtained as a pale yellow oil. $[\alpha]_{\mathrm{D}}^{20}=-2.9\left(c 1.9, \mathrm{CH}_{2} \mathrm{Cl}_{2}\right)$;

${ }^{1}$ H NMR (500 MHz, $\left.\mathrm{CDCl}_{3}\right): \delta 7.30-7.17(\mathrm{~m}, 10 \mathrm{H}), 4.43(\mathrm{ABq}, J=12.0 \mathrm{~Hz}, 1 \mathrm{H}), 4.18(\mathrm{ABq}, J=12.0$ $\mathrm{Hz}, 1 \mathrm{H}), 4.09$ (d, $J=7.2 \mathrm{~Hz}, 1 \mathrm{H}), 2.73-2.67(\mathrm{~m}, 1 \mathrm{H}), 1.60(\mathrm{~d}, J=2.4 \mathrm{~Hz}, 3 \mathrm{H}), 1.13(\mathrm{~d}, J=6.9 \mathrm{~Hz}, 3 \mathrm{H})$;

${ }^{13}$ C NMR (125 MHz, $\left.\mathrm{CDCl}_{3}\right): \delta 136.67,134.93,124.78,124.43,124.29,124.24,124.27,123.98,80.67$, 77.24, 74.32, 67.13, 30.28, 14.33, 0.00;

IR $\left(\right.$ neat $\left./ \mathrm{cm}^{-1}\right) v_{\max }: 3086,3064,3030,2970,2933,2929,2888,1496,1454,1071,1028,735,699$;

LCMS (ES+) $m / z$ calcd for $\mathrm{C}_{19} \mathrm{H}_{20} \mathrm{O}[\mathrm{M}+\mathrm{Na}]^{+} 287.14$, found 287.13 . 


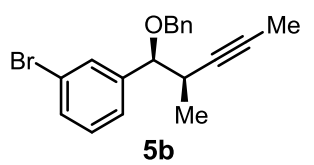

1-((1S,2R)-1-(benzyloxy)-2-methylpent-3-yn-1-yl)-3-bromobenzene

(5b):

Prepared by the general procedure using 3-bromobenzaldehyde, alkyne $\mathbf{5 b}(598 \mathrm{mg}, 85 \%$ yield, dr: 3.3:1) was obtained as a pale yellow oil. $[\alpha]_{\mathrm{D}}^{20}=-1.1\left(c 0.93, \mathrm{CH}_{2} \mathrm{Cl}_{2}\right)$;

${ }^{1}$ H NMR $\left(500 \mathrm{MHz}, \mathrm{CDCl}_{3}\right): \delta 7.47(\mathrm{~s}, 1 \mathrm{H}), 7.36(\mathrm{~d}, J=7.8 \mathrm{~Hz}, 1 \mathrm{H}), 7.27-7.13(\mathrm{~m}, 9 \mathrm{H}), 4.44(\mathrm{ABq}, J=$ $12.0 \mathrm{~Hz}, 1 \mathrm{H}), 4.18(\mathrm{ABq}, J=12.0 \mathrm{~Hz}, 1 \mathrm{H}), 4.03(\mathrm{~d}, J=7.3 \mathrm{~Hz}, 1 \mathrm{H}), 2.68-2.63(\mathrm{~m}, 1 \mathrm{H}), 1.62(\mathrm{~d}, J=2.4$ $\mathrm{Hz}, 3 \mathrm{H}), 1.12(\mathrm{~d}, J=6.9 \mathrm{~Hz}, 3 \mathrm{H})$;

${ }^{13}$ C NMR $\left(125 \mathrm{MHz}, \mathrm{CDCl}_{3}\right): \delta 139.24,134.57,127.41,127.39,126.06,124.91,124.37,124.21$, $122.89,118.73,80.15,76.80,67.46,30.34,14.44,0.00$;

IR $\left(\right.$ neat $\left./ \mathrm{cm}^{-1}\right) v_{\max }: 3088,3065,3034,2975,2917,2875,1595,1570,1497,1475,1454,1429,1203$, 1070, 784, 734, 696;

LCMS(ES+) $m / z$ calcd for $\mathrm{C}_{19} \mathrm{H}_{19} \mathrm{BrO}[\mathrm{M}+\mathrm{Na}]^{+} 365.05$, found 365.13 .

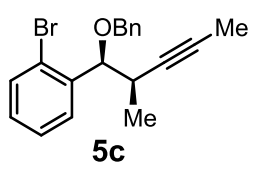

1-((1S,2R)-1-(benzyloxy)-2-methylpent-3-yn-1-yl)-2-bromobenzene (5c): Prepared by the general procedure using 3-bromobenzaldehyde, alkyne 5c (570 $\mathrm{mg}, 81 \%$ yield, dr: 4.0:1) was obtained as a pale yellow oil. $[\alpha]_{\mathrm{D}}^{20}=-4.9\left(c 1, \mathrm{CH}_{2} \mathrm{Cl}_{2}\right)$;

${ }^{1} \mathbf{H}$ NMR $\left(500 \mathrm{MHz}, \mathrm{CDCl}_{3}\right): \delta 7.57(\mathrm{dd}, J=7.8,1.7 \mathrm{~Hz}, 1 \mathrm{H}), 7.54(\mathrm{dd}, J=8.0,1.2 \mathrm{~Hz}, 1 \mathrm{H}), 7.37-7.28$ $(\mathrm{m}, 6 \mathrm{H}), 7.16(\mathrm{ddd}, J=8.0,7.3,1.8 \mathrm{~Hz}, 1 \mathrm{H}), 4.80(\mathrm{~d}, J=6.4 \mathrm{~Hz}, 1 \mathrm{H}), 4.47(\mathrm{ABq}, J=11.9 \mathrm{~Hz}, 1 \mathrm{H})$, $4.31(\mathrm{ABq}, J=11.9 \mathrm{~Hz}, 1 \mathrm{H}), 2.86-2.79(\mathrm{~m}, 1 \mathrm{H}), 1.72(\mathrm{~d}, J=2.4 \mathrm{~Hz}, 3 \mathrm{H}), 1.20(\mathrm{~d}, J=6.9 \mathrm{~Hz}, 3 \mathrm{H})$;

${ }^{13}$ C NMR $\left(125 \mathrm{MHz}, \mathrm{CDCl}_{3}\right): \delta 139.61,138.16,132.48,129.05,128.92,128.25,127.88,127.56$, $127.39,124.43,81.89,80.41,77.39,71.11,32.71,16.83,3.60$;

IR $\left(\right.$ neat $\left./ \mathrm{cm}^{-1}\right) v_{\max }: 3088,3065,3034,2975,2917,2875,1595,1570,1497,1475,1454,1429,1203$, 1070, 784, 734, 696;

LCMS(ES+) $m / z$ calcd for $\mathrm{C}_{19} \mathrm{H}_{19} \mathrm{BrO}[\mathrm{M}+\mathrm{Na}]^{+} 365.05$, found 365.15 .

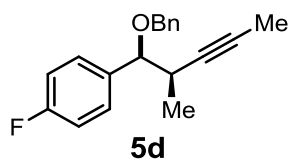

1-((1S,2R)-1-(benzyloxy)-2-methylpent-3-yn-1-yl)-4-fluorobenzene (5d): Prepared by the general procedure using 2,5-dimethoxybenzaldehyde, alkyne $\mathbf{5 d}$ (301 $\mathrm{mg}, 52 \%$ yield, 4.0:1) was obtained as a pale yellow oil. $[\alpha]_{\mathrm{D}}^{20}=-3.8\left(c 3.2, \mathrm{CH}_{2} \mathrm{Cl}_{2}\right)$;

${ }^{1} \mathbf{H}$ NMR $\left(500 \mathrm{MHz}, \mathrm{CDCl}_{3}\right)$ : $87.38-7.27(\mathrm{~m}, 7 \mathrm{H}), 7.07-7.04(\mathrm{~m}, 2 \mathrm{H}), 4.50(\mathrm{ABq}, J=12.0 \mathrm{~Hz}, 1 \mathrm{H}), 4.25$ $(\mathrm{ABq}, J=12.0 \mathrm{~Hz}, 1 \mathrm{H}), 4.14(\mathrm{~d}, J=7.4 \mathrm{~Hz}, 1 \mathrm{H}), 2.79-2.72(\mathrm{~m}, 1 \mathrm{H}), 1.69(\mathrm{dd}, J=2.4,0.6 \mathrm{~Hz}, 3 \mathrm{H}), 1.22$ (dd, $J=6.9,0.9 \mathrm{~Hz}, 3 \mathrm{H})$;

${ }^{13}$ C NMR (125 MHz, $\left.\mathrm{CDCl}_{3}\right): \delta 163.37,161.42,138.16,135.87,129.29,129.23,128.31,127.78$, $127.56,114.87,114.70,83.51,80.42,78.11,70.60,33.83,17.96,3.44$;

IR $\left(\right.$ neat $\left./ \mathrm{cm}^{-1}\right) v_{\max }: 3034,2972,2880,1606,1454,1223,1157,1074,835,737,698$; 
HRMS (ESI) $m / z$ calcd for $\mathrm{C}_{19} \mathrm{H}_{19} \mathrm{FO}[\mathrm{M}+\mathrm{H}]^{+} 283.1498$, found 283.1499 .

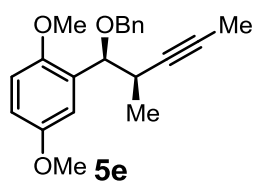

2-((1S,2R)-1-(benzyloxy)-2-methylpent-3-yn-1-yl)-1,4-dimethoxybenzene

(5e):

Prepared by the general procedure using 2,5-dimethoxybenzaldehyde, alkyne 5e $(521 \mathrm{mg}, 78 \%$ yield, dr: 3.0:1) was obtained as a pale yellow oil. $[\alpha]_{\mathrm{D}}^{20}=-0.2\left(c 0.9, \mathrm{CH}_{2} \mathrm{Cl}_{2}\right)$;

${ }^{1} \mathbf{H}$ NMR $\left(500 \mathrm{MHz}, \mathrm{CDCl}_{3}\right): 87.29-7.17(\mathrm{~m}, 5 \mathrm{H}), 7.03(\mathrm{~d}, J=1.7 \mathrm{~Hz}, 1 \mathrm{H}), 6.74-6.70(\mathrm{~m}, 2 \mathrm{H}), 4.75(\mathrm{~d}$, $J=5.6 \mathrm{~Hz}, 1 \mathrm{H}), 4.45(\mathrm{ABq}, J=12.1 \mathrm{~Hz}, 1 \mathrm{H}), 4.26(\mathrm{ABq}, J=12.1 \mathrm{~Hz}, 1 \mathrm{H}), 3.70(\mathrm{~s}, 3 \mathrm{H}), 3.68(\mathrm{~s}, 3 \mathrm{H})$, $1.67(\mathrm{~d}, J=2.4 \mathrm{~Hz}, 3 \mathrm{H}), 1.05(\mathrm{~d}, J=7.0 \mathrm{~Hz}, 3 \mathrm{H})$;

${ }^{13}$ C NMR (125 MHz, $\left.\mathrm{CDCl}_{3}\right)$ : $\delta 149.97,148.24,135.12,125.94,124.52,124.12,123.69,109.89$, 109.66, 107.83, 77.82, 73.46, 72.96, 67.38, 52.36, 52.01, 28.53, 12.91, 0.00;

IR $\left(\right.$ neat $\left./ \mathrm{cm}^{-1}\right) v_{\max }: 2937,2833,1497,1454,1276,1215,1048,1028,810,735,699$;

HRMS (ESI) $m / z$ calcd for $\mathrm{C}_{21} \mathrm{H}_{24} \mathrm{O}_{3}[\mathrm{M}+\mathrm{Na}]^{+} 347.1623$, found 347.1627 .

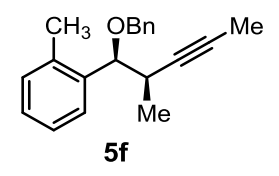

by the general procedure using o-tolualdehyde and 1 equivelant TMSOTf, alkyne $\mathbf{5 f}(286 \mathrm{mg}, 51 \%$ yield, dr: $>20: 1)$ was obtained as a colorless oil. $[\alpha]_{\mathrm{D}}^{20}=-7.2\left(c 2.5, \mathrm{CH}_{2} \mathrm{Cl}_{2}\right)$;

${ }^{1} \mathbf{H}$ NMR $\left(400 \mathrm{MHz}_{\mathrm{CDCl}}\right): \delta 7.52(\mathrm{~d}, J=7.6 \mathrm{~Hz}, 1 \mathrm{H}), 7.40-7.16(\mathrm{~m}, 8 \mathrm{H}), 4.54(\mathrm{~d}, J=7.6 \mathrm{~Hz}, 1 \mathrm{H})$, $4.49(\mathrm{ABq}, J=12.0 \mathrm{~Hz}, 1 \mathrm{H}), 4.23(\mathrm{ABq}, J=12.0 \mathrm{~Hz}, 1 \mathrm{H}), 2.82-2.74(\mathrm{~m}, 1 \mathrm{H}), 2.34(\mathrm{~s}, 3 \mathrm{H}), 1.68(\mathrm{~d}, J=$ $2.4 \mathrm{~Hz}, 3 \mathrm{H}), 1.31(\mathrm{~d}, J=6.8 \mathrm{~Hz}, 3 \mathrm{H})$;

${ }^{13} \mathbf{C}$ NMR $\left(100 \mathrm{MHz}, \mathrm{CDCl}_{3}\right): \delta 135.36,135.06,133.09,126.55,124.78,124.31,123.99,123.79$, $123.41,122.47,77.39,76.43,73.34,66.96,29.87,16.01,14.28,0.00$;

IR $\left(\right.$ neat $\left./ \mathrm{cm}^{-1}\right) v_{\max }: 3066,3029,2968,2930,1495,1454,1110,1064,757,726,697$;

$\mathbf{L C M S}(\mathrm{ES}+) m / z$ calcd for $\mathrm{C}_{20} \mathrm{H}_{22} \mathrm{O}[\mathrm{M}+\mathrm{H}]^{+} 279.17$, found 279.05 .

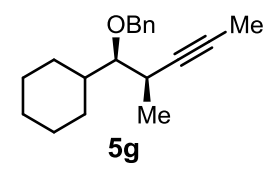

by the general procedure using cyclohexanecarbaldehyde, alkyne $\mathbf{5 g}$ (479 $\mathrm{mg}, 86 \%$ yield, dr: >20:1) was obtained as a colorless oil. $[\alpha]_{\mathrm{D}}^{20}=-0.5\left(c 1.6, \mathrm{CH}_{2} \mathrm{Cl}_{2}\right)$;

${ }^{1} \mathbf{H}$ NMR $\left(500 \mathrm{MHz}, \mathrm{CDCl}_{3}\right): \delta 7.31-7.17(\mathrm{~m}, 5 \mathrm{H}), 4.66(\mathrm{ABq}, J=11.0 \mathrm{~Hz}, 1 \mathrm{H}), 4.51(\mathrm{ABq}, J=11.0$ $\mathrm{Hz}, 1 \mathrm{H}), 3.06(\mathrm{dd}, J=11.6,6 \mathrm{~Hz}, 1 \mathrm{H}), 2.62-2.55(\mathrm{~m}, 1 \mathrm{H}), 1.81(\mathrm{~d}, J=12.7 \mathrm{~Hz}, 1 \mathrm{H}), 1.71(\mathrm{~d}, J=2.4 \mathrm{~Hz}$, $3 \mathrm{H}), 1.67-1.52(\mathrm{~m}, 5 \mathrm{H}), 1.12(\mathrm{~d}, J=6.9 \mathrm{~Hz}, 3 \mathrm{H}), 1.21-1.00(\mathrm{~m}, 5 \mathrm{H})$; 
${ }^{13}$ C NMR $\left(125 \mathrm{MHz}, \mathrm{CDCl}_{3}\right): \delta 135.45,124.66,124.14,123.80,83.74,78.93,73.03,71.64,37.44$, 27.02, 24.96, 24.28, 22.98, 22.95, 22.72, 13.31, 0.00;

IR $\left(\right.$ neat $\left./ \mathrm{cm}^{-1}\right) v_{\max }: 2929,2852,1497,1452,1110,1091,1057,732,696$;

HRMS (ESI) $m / z$ calcd for $\mathrm{C}_{19} \mathrm{H}_{26} \mathrm{O}[\mathrm{M}+\mathrm{H}]^{+} 271.2062$, found 271.2053.

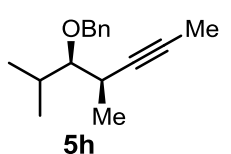

(((3R,4R)-2,4-dimethylhept-5-yn-3-yl)oxy)methyl)benzene (5h): Prepared by the general procedure using isobutyraldehyde, alkyne $\mathbf{5 h}$ (356 $\mathrm{mg}$, 75\% yield, dr: >20:1) was obtained as a colorless oil. $[\alpha]_{\mathrm{D}}^{20}=-2.8\left(c 1.2, \mathrm{CH}_{2} \mathrm{Cl}_{2}\right)$;

${ }^{1} \mathbf{H}$ NMR (400 MHz, $\left.\mathrm{CDCl}_{3}\right): 87.40-7.27(\mathrm{~m}, 5 \mathrm{H}), 4.76(\mathrm{ABq}, J=11.1 \mathrm{~Hz}, 1 \mathrm{H}), 4.63(\mathrm{ABq}, J=11.1$ $\mathrm{Hz}, 1 \mathrm{H}), 3.17-3.14(\mathrm{~m}, 1 \mathrm{H}), 2.68-2.60(\mathrm{~m}, 1 \mathrm{H}), 2.15-2.06(\mathrm{~m}, 1 \mathrm{H}), 1.81-1.80(\mathrm{~m}, 3 \mathrm{H}), 1.25-1.23(\mathrm{~m}$, $3 \mathrm{H}), 1.01-0.98(\mathrm{~m}, 6 \mathrm{H})$;

${ }^{13}$ C NMR (100 MHz, $\left.\mathrm{CDCl}_{3}\right): \delta 135.39,124.69,124.14,123.85,84.35,78.67,73.19,71.71,27.48$, 25.77, 16.84, 13.73, 13.56, 0.00;

IR $\left(\right.$ neat $\left./ \mathrm{cm}^{-1}\right) v_{\max }: 2966,2874,1454,1135,1094,1068,969,733,696$;

HRMS (ESI) $m / z$ calcd for $\mathrm{C}_{16} \mathrm{H}_{22} \mathrm{O}[\mathrm{M}+\mathrm{H}]^{+} 231.1749$, found 231.1745.

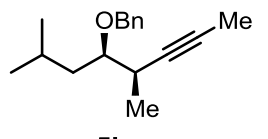

$5 \mathbf{i}$

((((4R,5R)-2,5-dimethyloct-6-yn-4-yl)oxy)methyl)benzene (5i): Prepared by the general procedure using isovaleraldehyde, alkyne $\mathbf{5 i}(332 \mathrm{mg}, 66 \%$ yield, dr: 3.0:1) was obtained as a colorless oil. $[\alpha]_{\mathrm{D}}^{20}=-3.1\left(c 0.9, \mathrm{CH}_{2} \mathrm{Cl}_{2}\right)$;

${ }^{1} \mathbf{H}$ NMR $\left(500 \mathrm{MHz}, \mathrm{CDCl}_{3}\right): \delta 7.38-7.28(\mathrm{~m}, 5 \mathrm{H}), 4.70(\mathrm{ABq}, J=11.4 \mathrm{~Hz}, 1 \mathrm{H}), 4.52(\mathrm{ABq}, J=11.4$ $\mathrm{Hz}, 1 \mathrm{H}), 3.38(\mathrm{ddd}, J=8.6,5.0,3.2 \mathrm{~Hz}, 1 \mathrm{H}), 2.77-2.70(\mathrm{~m}, 1 \mathrm{H}), 1.88-1.76(\mathrm{~m}, 1 \mathrm{H}), 1.81(\mathrm{~d}, J=2.4 \mathrm{~Hz}$, $3 \mathrm{H}), 1.66$ (ddd, $J=13.9,9.3,4.4 \mathrm{~Hz}, 1 \mathrm{H}), 1.31(\mathrm{ddd}, J=14.2,9.5,3.2 \mathrm{~Hz}, 1 \mathrm{H}), 1.17(\mathrm{~d}, J=7.0 \mathrm{~Hz}$, $3 \mathrm{H})$;

${ }^{13} \mathbf{C}$ NMR $\left(125 \mathrm{MHz}, \mathrm{CDCl}_{3}\right): \delta 138.75,128.28,127.97,127.88,127.49,81.32,80.23,76.99,71.87$, $41.09,30.16,24.43,23.84,21.91,17.31,3.64$;

IR $\left(\right.$ neat $\left./ \mathrm{cm}^{-1}\right) v_{\max }: 2956,2933,2920,2869,1497,1454,1368,1349,1098,734,697$;

HRMS (ESI) $m / z$ calcd for $\mathrm{C}_{17} \mathrm{H}_{24} \mathrm{O}[\mathrm{M}+\mathrm{H}]^{+} 245.1905$, found 245.1900.

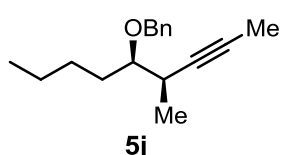

((((4R,5R)-4-methylnon-2-yn-5-yl)oxy)methyl)benzene $(\mathbf{5 j})$ : Prepared by the general procedure using pentanal, alkyne $\mathbf{5 j}$ (327 $\mathrm{mg}$, 65\% yield, dr: 2.0:1) was obtained as a colorless oil. $[\alpha]_{\mathrm{D}}^{20}=-1.9\left(c 1, \mathrm{CH}_{2} \mathrm{Cl}_{2}\right)$; 
${ }^{1} \mathbf{H}$ NMR $\left(400 \mathrm{MHz}, \mathrm{CDCl}_{3}\right): 87.38-7.26(\mathrm{~m}, 5 \mathrm{H}), 4.63(\mathrm{ABq}, J=11.5 \mathrm{~Hz}, 1 \mathrm{H}), 4.55(\mathrm{ABq}, J=11.5$ $\mathrm{Hz}, 1 \mathrm{H}), 3.31-3.26(\mathrm{~m}, 1 \mathrm{H}), 2.72-2.64(\mathrm{~m}, 1 \mathrm{H}), 1.81(\mathrm{~d}, J=2.4 \mathrm{~Hz}, 3 \mathrm{H}), 1.72-1.62(\mathrm{~m}, 2 \mathrm{H}), 1.37-1.27$ $(\mathrm{m}, 4 \mathrm{H}), 1.19(\mathrm{~d}, J=7.0 \mathrm{~Hz}, 2 \mathrm{H}), 0.91(\mathrm{t}, J=7.1 \mathrm{~Hz}, 3 \mathrm{H})$;

${ }^{13} \mathrm{C}$ NMR $\left(100 \mathrm{MHz}, \mathrm{CDCl}_{3}\right): \delta 135.14,124.66,124.29,123.86,78.66,77.76,73.28,68.39,27.71$, 26.52, 23.86, 19.23, 13.96, 10.48, 0.00;

IR $\left(\right.$ neat $\left./ \mathrm{cm}^{-1}\right) v_{\max }: 2957,2933,2858,1497,1454,1375,1347,1302,1205,1096,734,697$;

HRMS (ESI) $\mathrm{m} / z$ calcd for $\mathrm{C}_{17} \mathrm{H}_{24} \mathrm{O}[\mathrm{M}+\mathrm{H}]^{+} 245.1905$, found 245.1911 .

iv. Supplementary Table S2: Titanocyclopropene Complex Generation Optimization

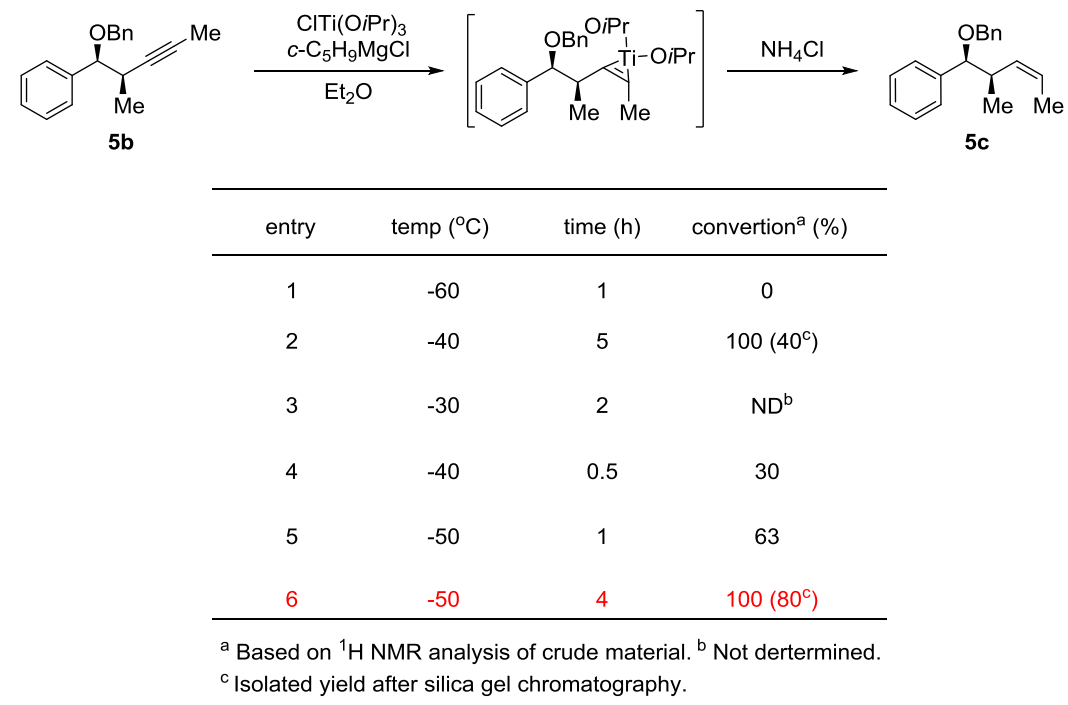

The efficient generation of titanocyclopropene complex is crucial to the success of alkyne-alkyne reductive coupling. Reaction condition evaluation (Table S2) identified that metallacyclopropene was effectively generated with $100 \%$ conversion and an $80 \%$ isolated yield, when the reaction was conducted at $50{ }^{\circ} \mathrm{C}$ for 4 hours. Upon hydrolytic quench, $(Z)$-alkene was cleanly formed.

v. Supplementary Table S3: Optimization for Alkyne-Alkyne Reductive Coupling between 5b and Methyl Propiolate 


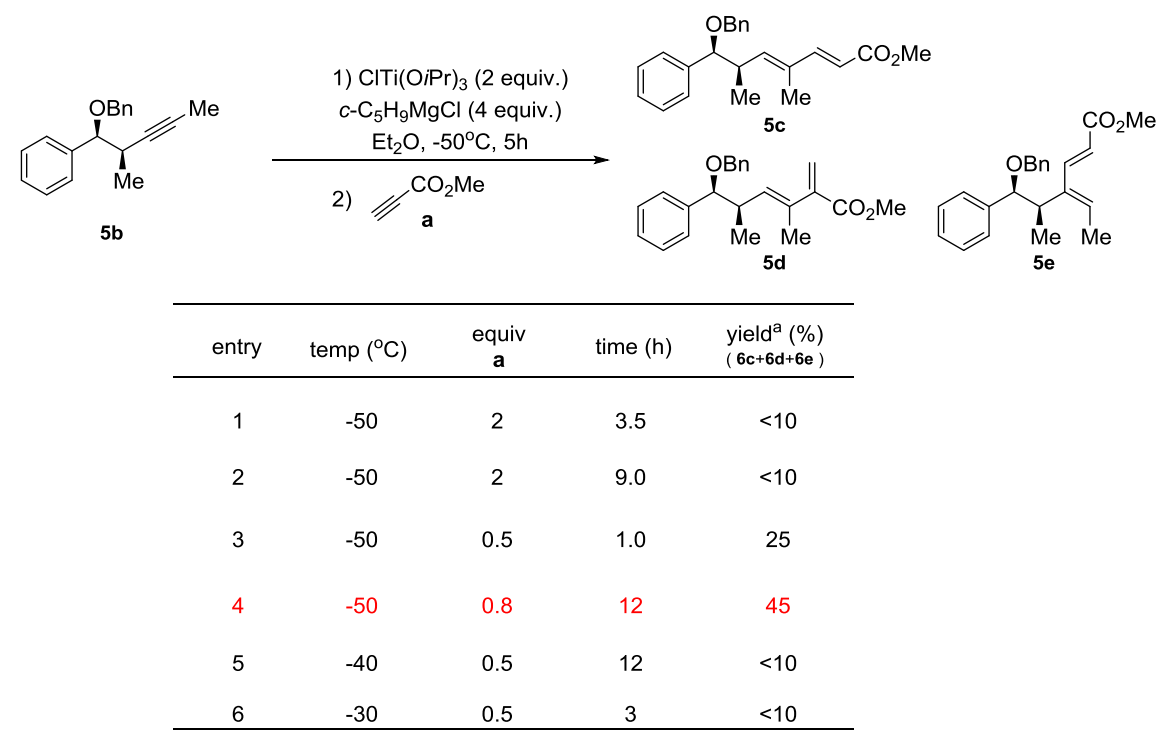

a) Yields refer to combined yields of regioisomers after silica gel chromatography

The initial trial employed Micalizio's condition ${ }^{5}$ for non-directed alkyne-alkyne reductive coupling (Table S3, entry 6), which resulted in significant decomposition. A screen of temperature, stoichiometry of methyl propiolate, and reaction time identified $-50{ }^{\circ} \mathrm{C}, 0.8$ equivalent of methyl propiolate, and 12 hour reaction time as optimal condition.

\section{vi. Alkyne-Alkyne Reductive Coupling}

General procedure for alkyne-alkyne reductive coupling between internal alkyne 5 and acetylenic esters: To a solution of internal alkyne $5\left(0.14 \mathrm{mmol}, 1\right.$ equiv) in $\mathrm{Et}_{2} \mathrm{O}(2.4 \mathrm{~mL})$ at $-78{ }^{\circ} \mathrm{C}$ was added sequentially $\mathrm{ClTi}(\mathrm{OiPr})_{3}\left(1.0 \mathrm{M}\right.$ in hexanes, $0.28 \mathrm{~mL}, 0.28 \mathrm{mmol}, 2$ equiv) and $c-\mathrm{C}_{5} \mathrm{H}_{9} \mathrm{MgCl}(2.0 \mathrm{M}$ in $\mathrm{Et}_{2} \mathrm{O}, 0.28 \mathrm{~mL}, 0.56 \mathrm{mmol}, 4$ equiv) dropwise. The resulting yellow solution turned brown while warming slowly to $-50{ }^{\circ} \mathrm{C}$ over $45 \mathrm{~min}$. The reaction mixture was placed in $-50{ }^{\circ} \mathrm{C}$ chiller and stirred for 4 hours, while solution turned to be black and heterogenous. It was then cooled to $-78{ }^{\circ} \mathrm{C}$, to which acetylenic ester $(0.11 \mathrm{mmol}, 0.8$ equiv) was added dropwise. The resulting mixture was again warmed to $-50{ }^{\circ} \mathrm{C}$ over $45 \mathrm{~min}$, which was subsequently placed into $-50{ }^{\circ} \mathrm{C}$ chiller and stirred for 12 hours. The reaction was quenched with saturated aqueous $\mathrm{NH}_{4} \mathrm{Cl}$ solution $(3 \mathrm{~mL})$. The mixture was warmed to r.t. before extractive isolation with diethyl ether $(3 \times 3 \mathrm{~mL})$. The combined organic layers were dried over $\mathrm{MgSO}_{4}$, filtered, and concentrated. Purification over silica gel chromatography (EtOAc/Hexanes, or $\mathrm{CH}_{2} \mathrm{Cl}_{2}$ /Hexanes) afforded coupled product 6 .<smiles>CC(=O)/C=C/C(=[W])O[Na]</smiles>

Methyl $\quad(2 E, 4 E, 6 R, 7 S)-7-(b e n z y l o x y)-4,6-d i m e t h y l-7-p h e n y l h e p t a-2,4-$ dienoate 6a: Prepared by the general procedure using internal alkyne 5a, diene 6a (17 mg, 45\%) was obtained as a pale yellow oil in a 3:1.2:1 mixture of regioisomers. $[\alpha]_{\mathrm{D}}^{20}=+2.8\left(c 0.4, \mathrm{CH}_{2} \mathrm{Cl}_{2}\right)$;

${ }^{5}$ Shimp, H. L.; Micalizio, G. C. Org. Lett. 2005, 7, 5111. 
${ }^{1} \mathbf{H}$ NMR $\left(500 \mathrm{MHz}, \mathrm{C}_{6} \mathrm{D}_{6}\right): \delta 7.41(\mathrm{~d}, J=15.7 \mathrm{~Hz}, 1 \mathrm{H}), 7.23-6.99(\mathrm{~m}, 10 \mathrm{H}), 5.73(\mathrm{~d}, J=15.6 \mathrm{~Hz}, 1 \mathrm{H})$, $5.39(\mathrm{~d}, J=9.3 \mathrm{~Hz}, 1 \mathrm{H}), 4.35(\mathrm{~d}, J=12.0 \mathrm{~Hz}, 1 \mathrm{H}), 4.02(\mathrm{~d}, J=11.9 \mathrm{~Hz}, 1 \mathrm{H}), 3.87(\mathrm{~d}, J=7.2 \mathrm{~Hz}, 1 \mathrm{H})$, $3.40(\mathrm{~s}, 3 \mathrm{H}), 2.79-2.71(\mathrm{~m}, 1 \mathrm{H}), 1.19(\mathrm{~d}, J=1.2 \mathrm{~Hz}, 3 \mathrm{H}), 1.02(\mathrm{~d}, J=6.6 \mathrm{~Hz}, 3 \mathrm{H})$;

${ }^{13}$ C NMR $\left(125 \mathrm{MHz}, \mathrm{C}_{6} \mathrm{D}_{6}\right): \delta 166.97,149.25,143.11,140.51,138.68,132.69,128.17,128.00,127.50$, $127.34,127.22,115.98,84.29,70.36,50.60,40.68,16.34,11.67$;

IR (neat/cm $\left.{ }^{-1}\right) v_{\max }: 3032,2951,2869,1720,1625,1494,1454,1313,1275,1171,1069,761,700$;

HRMS (ESI) $m / z$ calcd for $\mathrm{C}_{23} \mathrm{H}_{26} \mathrm{O}_{3}[\mathrm{M}+\mathrm{Na}]^{+} 373.1780$, found 373.1770 .

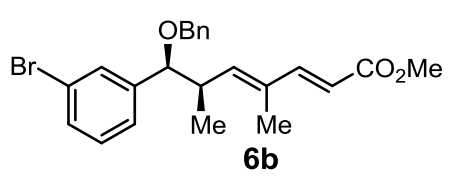

Methyl (2E,4E,6R,7S)-7-(benzyloxy)-7-(3-bromophenyl)-4,6dimethylhepta-2,4-dienoate (6b): Prepared by the general procedure using internal alkyne $\mathbf{5 b}$, diene 6b $(22 \mathrm{mg}, 46 \%)$ was obtained as a pale yellow oil in a 2.2:0:1 mixture of regioisomers. $[\alpha]_{\mathrm{D}}^{20}=+0.3(c$ $0.5, \mathrm{CH}_{2} \mathrm{Cl}_{2}$ );

${ }^{1}$ H NMR $\left(500 \mathrm{MHz}, \mathrm{C}_{6} \mathrm{D}_{6}\right): 87.40-7.37(\mathrm{~m}, 2 \mathrm{H}), 7.19-7.04(\mathrm{~m}, 8 \mathrm{H}), 5.74(\mathrm{~d}, J=15.7 \mathrm{~Hz}, 1 \mathrm{H}), 5.26(\mathrm{~d}, J$ $=10.0 \mathrm{~Hz}, 1 \mathrm{H}), 4.24(\mathrm{~d}, J=12.0 \mathrm{~Hz}, 1 \mathrm{H}), 3.91(\mathrm{~d}, J=12.0 \mathrm{~Hz}, 1 \mathrm{H}), 3.70(\mathrm{~d}, J=7.1 \mathrm{~Hz}, 1 \mathrm{H}), 3.40(\mathrm{~s}$, $3 \mathrm{H}), 2.62-2.55(\mathrm{~m}, 1 \mathrm{H}), 1.13(\mathrm{~s}, 3 \mathrm{H}), 0.93(\mathrm{~d}, J=6.6 \mathrm{~Hz}, 3 \mathrm{H})$,

${ }^{13}$ C NMR (125 MHz, $\left.\mathrm{C}_{6} \mathrm{D}_{6}\right): \delta 166.92,148.97,143.24,142.30,138.21,132.92,130.54,130.21,129.56$, $128.22,127.94,127.58,125.76,122.45,116.28,83.42,70.58,50.62,40.47,16.07,11.64$;

IR (neat/cm $\left.{ }^{-1}\right) v_{\max }: 3065,3028,2949,2927,2871,1719,1625,1571,1454,1433,1311,1270,1192$, $1171,1070,846,785,736,699$;

HRMS (ESI) $m / z$ calcd for $\mathrm{C}_{23} \mathrm{H}_{25} \mathrm{BrO}_{3}[\mathrm{M}+\mathrm{H}]^{+} 429.1065$, found 429.1076.

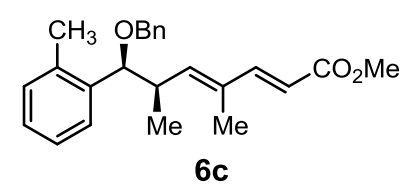

Methyl (2E,4E,6R,7S)-7-(benzyloxy)-4,6-dimethyl-7-(o-tolyl)hepta-2,4dienoate (6c): Prepared by the general procedure using internal alkyne 5c, diene $\mathbf{6 c}(18 \mathrm{mg}, 46 \%)$ was obtained as a pale yellow oil in a 4.7:1.7:1 mixture of regioisomers. $[\alpha]_{\mathrm{D}}^{20}=+0.75\left(c 0.7, \mathrm{CH}_{2} \mathrm{Cl}_{2}\right)$;

${ }^{1}$ H NMR $\left(500 \mathrm{MHz}, \mathrm{C}_{6} \mathrm{D}_{6}\right): \delta 7.43(\mathrm{dd}, J=15.65,0.75 \mathrm{~Hz}, 1 \mathrm{H}), 7.41(\mathrm{dd}, J=7.6,1.3 \mathrm{~Hz}), 7.18-7.14(\mathrm{~m}$, 2H), 7.08-6.95 (m, 4H), 6.88-6.87 (m, 1H), $5.74(\mathrm{dd}, J=15.7,0.6 \mathrm{~Hz}, 1 \mathrm{H}), 4.32(\mathrm{~d}, J=11.9 \mathrm{~Hz}, 1 \mathrm{H})$, $4.20(\mathrm{~d}, J=7.0 \mathrm{~Hz}, 1 \mathrm{H}), 3.96(\mathrm{~d}, J=12.0 \mathrm{~Hz}, 1 \mathrm{H}), 3.40(\mathrm{~s}, 3 \mathrm{H}), 2.80-2.73(\mathrm{~m}, 1 \mathrm{H}), 1.96(\mathrm{~s}, 3 \mathrm{H}), 1.15$ $(\mathrm{d}, J=1.2 \mathrm{~Hz}, 3 \mathrm{H}), 1.08(\mathrm{dd}, J=6.8,1.0 \mathrm{~Hz}, 3 \mathrm{H})$;

${ }^{13}$ C NMR (125 MHz, $\left.\mathrm{C}_{6} \mathrm{D}_{6}\right): \delta 166.92,149.18,143.16,138.69,135.60,132.31,130.26,128.14,127.93$, $127.34,127.17,127.14,125.86,116.14,80.08,70.21,50.58,40.07,19.00,15.94,11.55$;

IR $\left(\right.$ neat $\left./ \mathrm{cm}^{-1}\right) v_{\max }: 2947,1718,1622,1454,1311,1170,1067$;

HRMS (ESI) $m / z$ calcd for $\mathrm{C}_{24} \mathrm{H}_{28} \mathrm{O}_{3}[\mathrm{M}+\mathrm{Na}]^{+} 387.1936$, found 387.1921 . 
<smiles>CC(=O)/C=C/C(C)=C/[C@H](Cc1ccccc1)[C@H](C)c1ccccc1Br</smiles>

2,4-dienoate (6d): Prepared by the general procedure using internal alkyne 5d, diene $6 \mathbf{d}(22 \mathrm{mg}, 46 \%)$ was obtained as a pale yellow oil in a 4.7:1.7:1 mixture of regioisomers. $[\alpha]_{\mathrm{D}}^{20}=+0.4\left(c 0.5, \mathrm{CH}_{2} \mathrm{Cl}_{2}\right)$;

${ }^{1} \mathbf{H}$ NMR $\left(500 \mathrm{MHz}, \mathrm{C}_{6} \mathrm{D}_{6}\right): \delta 7.53(\mathrm{~d}, J=16.3 \mathrm{~Hz}, 1 \mathrm{H}), 7.38(\mathrm{~d}, J=9.4 \mathrm{~Hz}, 1 \mathrm{H}), 7.24(\mathrm{dd}, J=8.0,1.2$ $\mathrm{Hz}, 1 \mathrm{H}), 7.17-7.11(\mathrm{~m}, 4 \mathrm{H}), 7.07-7.03(\mathrm{~m}, 1 \mathrm{H}), 6.89(\mathrm{td}, J=7.4,0.9 \mathrm{~Hz}, 1 \mathrm{H}), 5.84(\mathrm{~d}, J=10 \mathrm{~Hz}, 1 \mathrm{H})$, $5.82(\mathrm{~d}, J=15.6 \mathrm{~Hz}, 1 \mathrm{H}), 4.65(\mathrm{~d}, J=5.6 \mathrm{~Hz}, 1 \mathrm{H}), 4.20(\mathrm{ABq}, J=11.8 \mathrm{~Hz}, 1 \mathrm{H}), 3.95(\mathrm{ABq}, J=11.8$ $\mathrm{Hz}, 1 \mathrm{H}), 3.41(\mathrm{~s}, 3 \mathrm{H}), 2.89-2.82(\mathrm{~m}, 1 \mathrm{H}), 1.35-1.33(\mathrm{~m}, 3 \mathrm{H}), 0.98(\mathrm{~d}, J=6.7 \mathrm{~Hz}, 3 \mathrm{H})$;

${ }^{13}$ C NMR (125 MHz, $\left.\mathrm{C}_{6} \mathrm{D}_{6}\right): \delta 166.87,149.27,142.97,139.87,138.18,132.61,128.75,128.73,128.14$, $127.75,127.51,127.10,123.66,116.29,82.16,70.95,50.56,39.33,14.81,1179$;

IR $\left(\right.$ neat $\left./ \mathrm{cm}^{-1}\right) v_{\max }: 3064,3032,2949,2872,1720,1624,1436,1311,1270,1170,1027,983,849,756$, 699;

HRMS (ESI) $m / z$ calcd for $\mathrm{C}_{23} \mathrm{H}_{25} \mathrm{BrO}_{3}[\mathrm{M}+\mathrm{H}]^{+} 429.1065$, found 429.1062.

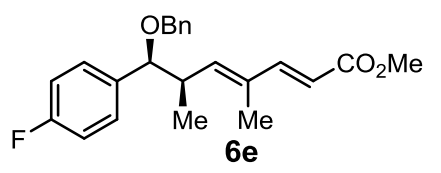

Methyl (2E,4E,6R,7S)-7-(benzyloxy)-7-(4-fluorophenyl)-4,6dimethylhepta-2,4-dienoate (6e): Prepared by the general procedure using internal alkyne 5e, diene $6 \mathbf{e}$ $(20 \mathrm{mg}, 50 \%)$ was obtained as a pale yellow oil in a 10:1:0 mixture of regioisomers. $[\alpha]_{\mathrm{D}}^{20}=+2.8(c 0.7$, $\mathrm{CH}_{2} \mathrm{Cl}_{2}$ );

${ }^{1} \mathbf{H}$ NMR $\left(500 \mathrm{MHz}, \mathrm{C}_{6} \mathrm{D}_{6}\right): \delta 7.41(\mathrm{dd}, J=15.7,0.7 \mathrm{~Hz}, 1 \mathrm{H}), 7.18-7.13(\mathrm{~m}, 4 \mathrm{H}), 7.09-7.06(\mathrm{~m}, 1 \mathrm{H})$, 6.86-6.82 (m, 2H), 6.74-6.68 (m, 2H), $5.75(\mathrm{dd}, J=15.7,0.5 \mathrm{~Hz}, 1 \mathrm{H}), 4.26(\mathrm{ABq}, J=12.0 \mathrm{~Hz}, 1 \mathrm{H})$, $3.95(\mathrm{ABq}, J=12.0 \mathrm{~Hz}, 1 \mathrm{H}), 3.75(\mathrm{~d}, J=7.4 \mathrm{~Hz}, 1 \mathrm{H}), 3.41(\mathrm{~s}, 3 \mathrm{H}), 2.69-2.61(\mathrm{~m}, 1 \mathrm{H}), 1.16(\mathrm{~d}, J=1.2$ $\mathrm{Hz}, 3 \mathrm{H}), 0.98(\mathrm{~d}, J=6.6 \mathrm{~Hz}, 3 \mathrm{H})$;

${ }^{13}$ C NMR $\left(125 \mathrm{MHz}, \mathrm{C}_{6} \mathrm{D}_{6}\right): \delta 166.95,163.22,161.27,149.05,142.58,138.45,136.17,136.14,132.81$, $128.78,128.72$, 128.21, 127.50, 116.20, 114.90, 114.73, 83.56, 70.32, 50.62, 40.62, 16.35, 11.66;

IR $\left(\right.$ neat $\left./ \mathrm{cm}^{-1}\right) v_{\max }: 3063,3032,2951,2867,1719,1622,1605,1508,1454,1434,1312,1274,1221$, 1193, 1169, 1071, 1015, 983, 833, 736, 698;

HRMS (ESI) $m / z$ calcd for $\mathrm{C}_{23} \mathrm{H}_{25} \mathrm{FO}_{3}[\mathrm{M}+\mathrm{H}]^{+} 369.1866$, found 369.1855 .

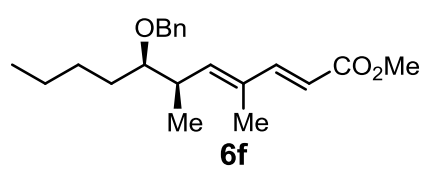

Methyl $\quad(2 E, 4 E, 6 R, 7 R)-7-($ benzyloxy)-4,6-dimethylundeca-2,4-dienoate (6f): Prepared by the general procedure using internal alkyne $\mathbf{5 f}$, diene $\mathbf{6 f}(17 \mathrm{mg}, 49 \%)$ was obtained as a colorless oil in a 10.5:1:2.5 mixture of regioisomers. $[\alpha]_{\mathrm{D}}^{20}=+1.6\left(c 0.3, \mathrm{CH}_{2} \mathrm{Cl}_{2}\right)$;

${ }^{1} \mathbf{H}$ NMR $\left(500 \mathrm{MHz}, \mathrm{C}_{6} \mathrm{D}_{6}\right): \delta 7.53(\mathrm{dd}, J=15.7,0.8 \mathrm{~Hz}, 1 \mathrm{H}), 7.28-7.24(\mathrm{~m}, 2 \mathrm{H}), 7.17-7.13(\mathrm{~m}, 2 \mathrm{H})$, 7.09-7.06 (m, 1H), $5.87(\mathrm{dd}, J=15.7,0.6 \mathrm{~Hz}, 1 \mathrm{H}), 5.50-5.48(\mathrm{~m}, 1 \mathrm{H}), 4.34(\mathrm{ABq}, J=11.6 \mathrm{~Hz}, 1 \mathrm{H})$, 
$4.26(\mathrm{ABq}, J=11.6 \mathrm{~Hz}, 1 \mathrm{H}), 3.45(\mathrm{~s}, 3 \mathrm{H}), 3.00-2.97(\mathrm{~m}, 1 \mathrm{H}), 2.63-2.55(\mathrm{~m}, 1 \mathrm{H}), 1.46(\mathrm{~s}, 3 \mathrm{H}), 0.91(\mathrm{~d}, J$ $=6.8 \mathrm{~Hz}, 3 \mathrm{H}), 0.82(\mathrm{t}, J=7.2 \mathrm{~Hz}, 3 \mathrm{H})$;

${ }^{13}$ C NMR $\left(125 \mathrm{MHz}, \mathrm{C}_{6} \mathrm{D}_{6}\right): \delta 167.02,149.48,144.33,139.14,131.98,128.14,127.40,127.26,115.95$, 82.58, 71.73, 50.63, 36.91, 31.22, 27.44, 22.87, 15.91, 13.91, 11.92;

IR $\left(\right.$ neat $\left./ \mathrm{cm}^{-1}\right) v_{\max }: 2956,2935,2873,1720,1625,1255,1435,1312,1272,1170,1069,984,848,736$, 699;

HRMS (ESI) $m / z$ calcd for $\mathrm{C}_{21} \mathrm{H}_{30} \mathrm{O}_{3}[\mathrm{M}+\mathrm{H}]^{+} 331.2273$, found 331.2271.

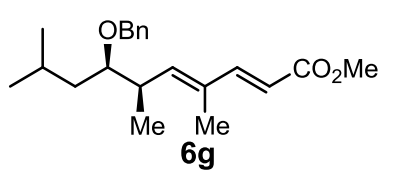
Prepared by the general procedure using internal alkyne $\mathbf{5 g}$, diene $\mathbf{6 g}$ (15 $\mathrm{mg}, 43 \%$ ) was obtained as a colorless oil in a 4.6:1:0 mixture of regioisomers. $[\alpha]_{\mathrm{D}}^{20}=+1.3\left(c 0.3, \mathrm{CH}_{2} \mathrm{Cl}_{2}\right)$;

${ }^{1} \mathbf{H}$ NMR $\left(400 \mathrm{MHz}, \mathrm{C}_{6} \mathrm{D}_{6}\right): \delta 7.15(\mathrm{~d}, J=15.7 \mathrm{~Hz}, 1 \mathrm{H}), 6.87(\mathrm{~d}, J=7.0 \mathrm{~Hz}, 2 \mathrm{H}), 6.88-6.65(\mathrm{~m}, 3 \mathrm{H})$, $5.49(\mathrm{~d}, J=15.7 \mathrm{~Hz}, 1 \mathrm{H}), 5.16(\mathrm{~d}, J=10.0 \mathrm{~Hz}, 1 \mathrm{H}), 3.90(\mathrm{~d}, J=2.0 \mathrm{~Hz}, 2 \mathrm{H}), 3.05(\mathrm{~s}, 3 \mathrm{H}), 2.77-2.72$ $(\mathrm{m}, 1 \mathrm{H}), 2.25-2.15(\mathrm{~m}, 1 \mathrm{H}), 1.35-1.25(\mathrm{~m}, 1 \mathrm{H}), 1.07(\mathrm{~d}, J=1.2 \mathrm{~Hz}, 3 \mathrm{H}), 1.02-0.99(\mathrm{~m}, 1 \mathrm{H}), 0.74-0.68$ $(\mathrm{m}, 1 \mathrm{H}), 0.48(\mathrm{~d}, J=6.8 \mathrm{~Hz}, 3 \mathrm{H}), 0.41(\mathrm{~d}, J=6.7 \mathrm{~Hz}, 3 \mathrm{H}), 0.39(\mathrm{~d}, J=6.6 \mathrm{~Hz}, 3 \mathrm{H})$;

${ }^{13}$ C NMR (100 MHz, $\left.\mathrm{C}_{6} \mathrm{D}_{6}\right): \delta 167.05,149.50,144.32,139.07,132.19,128.15,127.54,127.28,115.96$, 80.96, 71.65, 50.66, 40.96, 36.87, 24.61, 23.41, 22.05, 15.69, 11.99;

IR $\left(\right.$ neat $\left./ \mathrm{cm}^{-1}\right) v_{\max }: 2956,2870,1721,1625,1454,1435,1311,1273,1169,1094,1028,983,848,736$, 698 ;

HRMS (ESI) $m / z$ calcd for $\mathrm{C}_{21} \mathrm{H}_{30} \mathrm{O}_{3}[\mathrm{M}+\mathrm{H}]^{+} 331.2273$, found 331.2273.

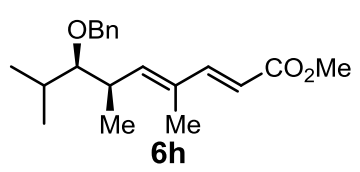

Prepared by the general procedure using internal alkyne $\mathbf{5 h}$, diene $\mathbf{6 h}(24 \mathrm{mg}, 62 \%)$ was obtained as a colorless oil in a 20:0:0 mixture of regioisomers. $[\alpha]_{\mathrm{D}}^{20}=+2.1\left(c 1.1, \mathrm{CH}_{2} \mathrm{Cl}_{2}\right)$;

${ }^{1} \mathbf{H}$ NMR $\left(500 \mathrm{MHz}, \mathrm{C}_{6} \mathrm{D}_{6}\right): \delta 7.51(\mathrm{dd}, J=15.7,0.7 \mathrm{~Hz}, 1 \mathrm{H}), 7.29(\mathrm{dd}, J=7.9,1.0 \mathrm{~Hz}, 2 \mathrm{H}), 7.18-7.15$ $(\mathrm{m}, 2 \mathrm{H}), 7.10-7.07(\mathrm{~m}, 1 \mathrm{H}), 5.88(\mathrm{~d}, J=15.3 \mathrm{~Hz}, 1 \mathrm{H}), 5.40(\mathrm{~d}, J=10.1 \mathrm{~Hz}, 2 \mathrm{H}), 4.37(\mathrm{ABq}, J=11.4$ $\mathrm{Hz}, 1 \mathrm{H}), 4.34(\mathrm{ABq}, J=11.4 \mathrm{~Hz}, 1 \mathrm{H}), 3.46(\mathrm{~s}, 3 \mathrm{H}), 2.70(\mathrm{dd}, J=7.2,4.2 \mathrm{~Hz}, 1 \mathrm{H}), 2.65-2.57(\mathrm{~m}, 1 \mathrm{H})$, $1.62-1.56(\mathrm{~m}, 1 \mathrm{H}), 1.44(\mathrm{~d}, J=1.2 \mathrm{~Hz}, 1 \mathrm{H}), 0.92(\mathrm{~d}, J=6.6 \mathrm{~Hz}, 3 \mathrm{H}), 0.85(\mathrm{~d}, J=5.9 \mathrm{~Hz}, 3 \mathrm{H}), 0.84(\mathrm{~d}, J$ $=6.0 \mathrm{~Hz}, 3 \mathrm{H})$;

${ }^{13}$ C NMR $\left(125 \mathrm{MHz}, \mathrm{C}_{6} \mathrm{D}_{6}\right): \delta 167.04,149.47,144.82,139.17,131.30,128.15,127.93,127.26,115.96$, 88.06, 75.25, 50.66, 36.73, 31.34, 20.26, 16.70, 16.13, 11.75;

IR $\left(\right.$ neat $\left./ \mathrm{cm}^{-1}\right) v_{\max }: 2965,2871,1720,1623,1497,1454,1434,1311,1270,1169,1067,1028,985$, 848, 734, 697;

HRMS (ESI) $m / z$ calcd for $\mathrm{C}_{20} \mathrm{H}_{28} \mathrm{O}_{3}[\mathrm{M}+\mathrm{H}]^{+} 317.2117$, found 317.2111. 


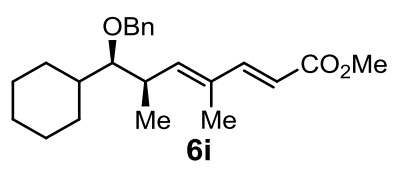

Methyl $(2 E, 4 E, 6 R, 7 R)-7-($ benzyloxy)-7-cyclohexyl-4,6-dimethylhepta-2,4dienoate (6i): Prepared by the general procedure using internal alkyne 5i, diene $\mathbf{6 i}(26 \mathrm{mg}, 67 \%)$ was obtained as a colorless oil in a 20:0:0 mixture of regioisomers. $[\alpha]_{\mathrm{D}}^{20}=+0.45\left(c 0.3, \mathrm{CH}_{2} \mathrm{Cl}_{2}\right)$;

${ }^{1} \mathbf{H}$ NMR $\left(500 \mathrm{MHz}, \mathrm{C}_{6} \mathrm{D}_{6}\right): \delta 7.52(\mathrm{dd}, J=15.7,0.6 \mathrm{~Hz}, 1 \mathrm{H}), 7.31-7.29(\mathrm{~m}, 2 \mathrm{H}), 7.18-7.15(\mathrm{~m}, 2 \mathrm{H})$, 7.10-7.07 (m, 1H), $5.89(\mathrm{~d}, J=15.6 \mathrm{~Hz}, 1 \mathrm{H}), 5.51(\mathrm{~d}, J=9.9 \mathrm{~Hz}, 1 \mathrm{H}), 4.39(\mathrm{ABq}, J=11.4 \mathrm{~Hz}, 1 \mathrm{H})$, $4.34(\mathrm{ABq}, J=11.4 \mathrm{~Hz}, 1 \mathrm{H}), 3.45(\mathrm{~s}, 3 \mathrm{H}), 2.75(\mathrm{dd}, J=6.4,4.9 \mathrm{~Hz}, 1 \mathrm{H}), 2.71-2.64(\mathrm{~m}, 1 \mathrm{H}), 1.78-1.47$ $(\mathrm{m}, 5 \mathrm{H}), 1.47(\mathrm{~d}, J=1.2 \mathrm{~Hz}, 3 \mathrm{H}), 1.40-1.33(\mathrm{~m}, 1 \mathrm{H}), 1.18-1.02(\mathrm{~m}, 5 \mathrm{H}), 0.94(\mathrm{~d}, J=6.6 \mathrm{~Hz}, 3 \mathrm{H})$;

${ }^{13}$ C NMR (125 MHz, $\left.\mathrm{C}_{6} \mathrm{D}_{6}\right)$ : $8167.00,149.44,145.21,139.20,131.30,128.16,127.23,115.98,87.72$, $75.23,50.65,41.56,35.91,30.72,27.75,26.51,26.30,15.63,11.81$;

IR $\left(\right.$ neat $\left./ \mathrm{cm}^{-1}\right) v_{\max }: 2929,2855,1743,1721,1624,1451,1312,1269,1170,1110,984,737,700$;

HRMS (ESI) $m / z$ calcd for $\mathrm{C}_{23} \mathrm{H}_{32} \mathrm{O}_{3}[\mathrm{M}+\mathrm{H}]^{+} 357.2430$, found 357.2440.

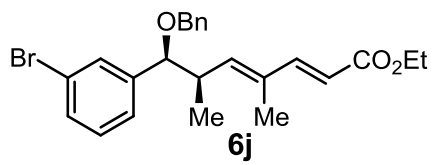

Ethyl

(2E,4E,6R,7S)-7-(benzyloxy)-7-(3-bromophenyl)-4,6dimethylhepta-2,4-dienoate (6j): Prepared by the general procedure using internal alkyne $\mathbf{5 b}$, diene $\mathbf{6 j}$ $(21 \mathrm{mg}, 44 \%)$ was obtained as a pale yellow oil in a 5:0:1 mixture of regioisomers. $[\alpha]_{\mathrm{D}}^{20}=+2.2(c 0.4$, $\mathrm{CH}_{2} \mathrm{Cl}_{2}$ );

${ }^{1}$ H NMR $\left(500 \mathrm{MHz}, \mathrm{C}_{6} \mathrm{D}_{6}\right): \delta 7.42(\mathrm{dd}, J=15.7,0.6 \mathrm{~Hz}, 1 \mathrm{H}), 7.39(\mathrm{t}, J=1.8 \mathrm{~Hz}, 1 \mathrm{H}), 7.17-7.04(\mathrm{~m}$, $6 \mathrm{H}), 6.82(\mathrm{~d}, J=7.7 \mathrm{~Hz}, 1 \mathrm{H}), 6.66(\mathrm{t}, J=7.8 \mathrm{~Hz}, 1 \mathrm{H}), 5.77(\mathrm{~d}, J=15.4 \mathrm{~Hz}, 1 \mathrm{H}), 5.26(\mathrm{~d}, J=10.0 \mathrm{~Hz}$, 1H), 4.03 (qd, $J=7.1,2.4 \mathrm{~Hz}, 2 \mathrm{H}), 3.91(\mathrm{~d}, J=12.0 \mathrm{~Hz}, 1 \mathrm{H}), 3.70(\mathrm{~d}, J=7.2 \mathrm{~Hz}, 1 \mathrm{H}), 2.63-2.56(\mathrm{~m}$, $1 \mathrm{H}), 1.14(\mathrm{~d}, J=1.2 \mathrm{~Hz}, 3 \mathrm{H}), 0.96(\mathrm{t}, J=7.1 \mathrm{~Hz}, 3 \mathrm{H}), 0.93(\mathrm{~d}, J=6.6 \mathrm{~Hz}, 3 \mathrm{H})$;

${ }^{13}$ C NMR (125 MHz, $\left.\mathrm{C}_{6} \mathrm{D}_{6}\right): \delta 166.61,148.82,143.35,142.21,138.32,133.05,130.62,130.28,129.64$, $128.31,128.03,127.67,125.88,122.54,116.87,83.53,70.66,59.76,40.58,16.20,14.09,11.77$;

IR $\left(\right.$ neat $\left./ \mathrm{cm}^{-1}\right) v_{\max }: 2978,2866,1713,1625,1455,1367,1308,1268,1176,1115,1070,982,786,698$;

HRMS (ESI) $\mathrm{m} / z$ calcd for $\mathrm{C}_{24} \mathrm{H}_{27} \mathrm{BrO}_{3}[\mathrm{M}+\mathrm{H}]^{+} 443.1222$, found 443.1213 .

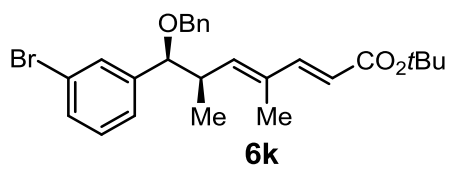

Tert-butyl $\quad(2 E, 4 E, 6 R, 7 S)-7-(b e n z y l o x y)-7-(3-b r o m o p h e n y l)-4,6-$ dimethylhepta-2,4-dienoate (6k): Prepared by the general procedure using internal alkyne $\mathbf{5 b}$, diene 6k $(28 \mathrm{mg}, 54 \%)$ was obtained as a pale yellow oil in a 20:0:0 mixture of regioisomers. $[\alpha]_{\mathrm{D}}^{20}=+1.8(c$ 1.0, $\mathrm{CH}_{2} \mathrm{Cl}_{2}$ );

${ }^{1}$ H NMR $\left(500 \mathrm{MHz}, \mathrm{C}_{6} \mathrm{D}_{6}\right): \delta 7.40-7.37(\mathrm{~m}, 2 \mathrm{H}), 7.13-7.03(\mathrm{~m}, 6 \mathrm{H}), 6.80(\mathrm{~d}, J=7.7 \mathrm{~Hz}, 1 \mathrm{H}), 6.65(\mathrm{t}, J$ $=7.8 \mathrm{~Hz}, 1 \mathrm{H}), 5.76(\mathrm{~d}, J=15.3 \mathrm{~Hz}, 1 \mathrm{H}), 5.25(\mathrm{~d}, J=10.1 \mathrm{~Hz}, 1 \mathrm{H}), 4.24(\mathrm{ABq}, J=12.0 \mathrm{~Hz}, 1 \mathrm{H}), 3.90$ 
$(\mathrm{ABq}, J=12.0 \mathrm{~Hz}, 1 \mathrm{H}), 3.69(\mathrm{~d}, J=7.2 \mathrm{~Hz}, 1 \mathrm{H}), 2.64-2.56(\mathrm{~m}, 1 \mathrm{H}), 1.40(\mathrm{~s}, 9 \mathrm{H}), 1.15(\mathrm{~d}, J=1.2 \mathrm{~Hz}$, $3 \mathrm{H}), 0.93(\mathrm{~d}, J=6.6 \mathrm{~Hz}, 1 \mathrm{H})$;

${ }^{13}$ C NMR $\left(125 \mathrm{MHz}, \mathrm{C}_{6} \mathrm{D}_{6}\right): \delta 166.03,147.90,143.30,141.56,138.26,132.97,130.51,130.14,129.53$, $128.21,127.58,125.85,122.45,118.59,83.50,79.11,70.56,40.47,27.87,16.18,11.77$;

IR $\left(\right.$ neat $\left./ \mathrm{cm}^{-1}\right) v_{\max }: 2994,1709,1624,1455,1392,1368,1314,1152,1068,983,700$;

HRMS (ESI) $m / z$ calcd for $\mathrm{C}_{26} \mathrm{H}_{31} \mathrm{BrO}_{3}[\mathrm{M}+\mathrm{Na}]^{+} 493.1354$, found 493.1367 .

\section{vii. Total Synthesis of ent-NFAT-68 and NFAT-68}

\section{a) Total Synthesis of ent-NFAT-68}

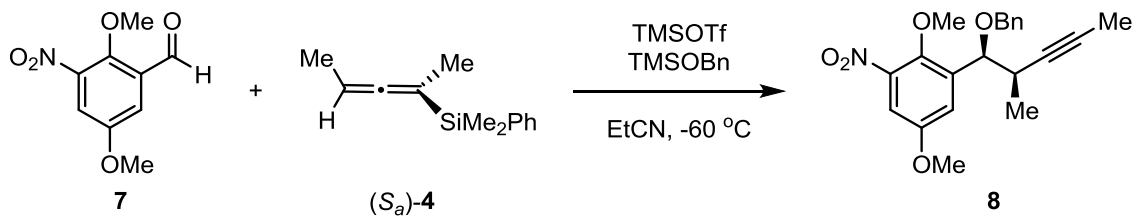

1-((1S,2R)-1-(benzyloxy)-2-methylpent-3-yn-1-yl)-2,5-dimethoxy-3-nitrobenzene (8): To a solution of aldehyde 7 ( $6 \mathrm{~g}, 28.4 \mathrm{mmol}, 1$ equiv), allenylsilane $\left(S_{a}\right)-4$ (6.2 g, $30.6 \mathrm{mmol}, 1.08$ equiv), and (benzyloxy)trimethylsilane $\left(6.4 \mathrm{~mL}, 32.7 \mathrm{mmol}, 1.1\right.$ equiv) in propionitrile $(300 \mathrm{~mL}, \mathrm{c}=0.1)$ at $-78{ }^{\circ} \mathrm{C}$ was added trimethylsilyl trifluoromethanesulfonate $(2.6 \mathrm{~mL}, 14.2 \mathrm{mmol}, 0.5$ equiv) dropwise. The resulting reaction mixture was stirred at $-60{ }^{\circ} \mathrm{C}$ for 24 hours. The reaction was quenched with $50 \mathrm{~mL}$ saturated $\mathrm{NaHCO}_{3}$ aqueous solution. It was then extract with ethyl acetate $(3 \times 300 \mathrm{~mL})$, and the combined organic layers were dried over $\mathrm{MgSO}_{4}$, filtered, and concentrated. Purification over silica gel chromatography $\left(60 \% \mathrm{CH}_{2} \mathrm{Cl}_{2} / \mathrm{Hexanes}\right)$ afforded product 8 (8 g, 76\% yield, dr: 5:1) as a yellow oil. $[\alpha]_{\mathrm{D}}^{20}=-3.9(c 2.9$, $\mathrm{CH}_{2} \mathrm{Cl}_{2}$ );

${ }^{1} \mathbf{H}$ NMR $\left(500 \mathrm{MHz}, \mathrm{CDCl}_{3}\right): \delta 7.35-7.28(\mathrm{~m}, 7 \mathrm{H}), 4.69(\mathrm{~d}, J=7.3 \mathrm{~Hz}, 1 \mathrm{H}), 4.46(\mathrm{ABq}, J=11.9 \mathrm{~Hz}$, $1 \mathrm{H}), 4.35(\mathrm{ABq}, J=11.9 \mathrm{~Hz}, 1 \mathrm{H}), 3.84(\mathrm{~s}, 3 \mathrm{H}), 3.80(\mathrm{~s}, 3 \mathrm{H}), 2.90-2.83(\mathrm{~m}, 1 \mathrm{H}), 1.68(\mathrm{~d}, J=2.4 \mathrm{~Hz}$, $3 \mathrm{H}), 1.23(\mathrm{~d}, J=6.9 \mathrm{~Hz}, 3 \mathrm{H})$;

${ }^{13} \mathbf{C}$ NMR $\left(125 \mathrm{MHz}, \mathrm{CDCl}_{3}\right): \delta 155.16,145.81,143.51,138.17,137.79,128.34,127.76,127.74$, $118.99,109.64,80.29,78.18,77.08,71.20,63.07,55.95,32.89,17.75,3.51$;

IR $\left(\right.$ neat $\left./ \mathrm{cm}^{-1}\right) v_{\max }: 2942,2918,1532,1482,1454,1429,1348,1307,1227,1051,996,737,699$;

HRMS (ESI) $m / z$ calcd for $\mathrm{C}_{21} \mathrm{H}_{23} \mathrm{NO}_{5}[\mathrm{M}+\mathrm{Na}]^{+} 392.1474$, found 392.1464.

Ee analysis of 8: Racemic propargylation product and $\mathbf{8}$ were run on chiral HPLC using a ChiralCel AD-H column, isocratic $1 \%$ ispropanol/hexanes at a flow rate of $1 \mathrm{~mL} / \mathrm{min}$. 8 was found to have $98 \%$ ee.<smiles>CC#CC(C)C(Cc1ccccc1)c1cc(OC)cc([N+](=O)[O-])c1OC</smiles> 

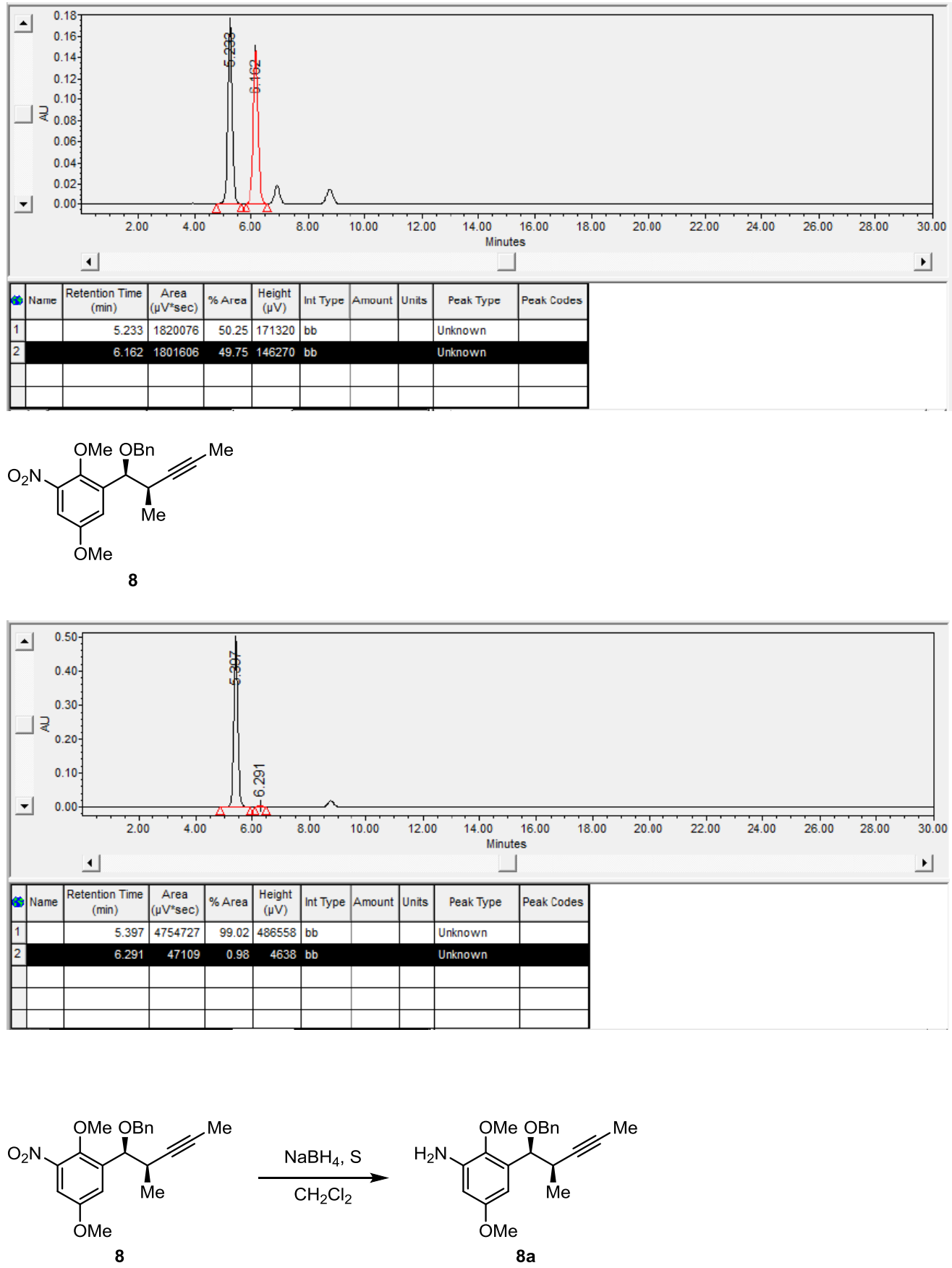

3-((1S,2R)-1-(benzyloxy)-2-methylpent-3-yn-1-yl)-2,5-dimethoxyaniline (8a): Sulfur powder (3.2 g, $100 \mathrm{mmol}, 4.6$ equiv) and sodium borohydride (1.28 g, $33.7 \mathrm{mmol}, 1.56$ equiv) were suspended in 460 $\mathrm{mL}$ THF and stirred for $30 \mathrm{~min}$, giving a yellow, homogenous solution. To the above solution was added 8 in $6 \mathrm{~mL}$ THF. The resulting mixture was heated at $70{ }^{\circ} \mathrm{C}$ for 4 hours until the complete consumption of $\mathbf{8}$ indicated by TLC. The reaction was cooled to room temperature and quenched with $160 \mathrm{~mL} 8 \%$ (w/v) $\mathrm{NaOH}$ aqueous solution. It was then extract with ethyl acetate $(3 \times 400 \mathrm{~mL})$, and the combined organic layers were dried over $\mathrm{MgSO}_{4}$, filtered, and concentrated. Purification over silica gel chromatography (24\% EtOAc/Hexanes) afforded $8 \mathbf{a}(6.29 \mathrm{~g}, 82 \%)$ as a brown oil. $[\alpha]_{\mathrm{D}}^{20}=-3.9\left(c 2.5, \mathrm{CH}_{2} \mathrm{Cl}_{2}\right)$;

${ }^{1}$ H NMR (500 MHz, $\left.\mathrm{CDCl}_{3}\right): \delta 7.41-7.24(\mathrm{~m}, 5 \mathrm{H}), 6.45(\mathrm{~d}, J=3.0 \mathrm{~Hz}, 1 \mathrm{H}), 6.28(\mathrm{~d}, J=3.0 \mathrm{~Hz}, 1 \mathrm{H})$, $4.62(\mathrm{~d}, J=7.0 \mathrm{~Hz}, 1 \mathrm{H}), 4.49(\mathrm{ABq}, J=12.0 \mathrm{~Hz}, 1 \mathrm{H}), 4.33(\mathrm{ABq}, J=12.0 \mathrm{~Hz}, 1 \mathrm{H}), 3.78(\mathrm{~s}, 2 \mathrm{H}), 3.75$ $(\mathrm{d}, J=1.7 \mathrm{~Hz}, 3 \mathrm{H}), 3.68(\mathrm{~s}, 3 \mathrm{H}), 2.92-2.86(\mathrm{~m}, 1 \mathrm{H}), 1.70(\mathrm{~d}, J=2.4 \mathrm{~Hz}, 3 \mathrm{H}), 1.24(\mathrm{~d}, J=6.9 \mathrm{~Hz}, 3 \mathrm{H})$; 
${ }^{13}$ C NMR $\left(125 \mathrm{MHz}, \mathrm{CDCl}_{3}\right): \delta 156.41,140.43,140.18,138.63,133.95,128.20,127.72,127.38$, $101.65,81.41,77.86,77.15,70.64,60.13,55.35,32.56,17.59,3.64$;

IR $\left(\right.$ neat $\left./ \mathrm{cm}^{-1}\right) v_{\max }: 3459,3365,2937,2836,1615,1495,1453,1350,1240,1197,1170,1045,1000$, 837, 738, 699;

HRMS (ESI) $m / z$ calcd for $\mathrm{C}_{21} \mathrm{H}_{25} \mathrm{NO}_{3}[\mathrm{M}+\mathrm{H}]^{+} 340.1913$, found 340.1927 .

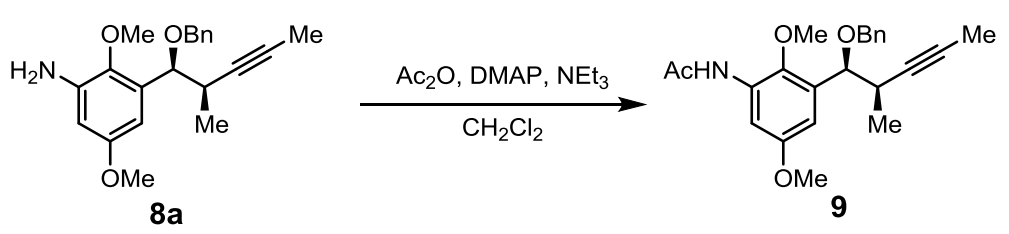

$N$-(3-((1S,2R)-1-(benzyloxy)-2-methylpent-3-yn-1-yl)-2,5-dimethoxyphenyl)acetamide (9): To a solution of $8 \mathbf{a}$ (6.29 g, $18.54 \mathrm{mmol}, 1$ equiv), acetic anhydride (4.5 mL, $37.08 \mathrm{mmol}, 2$ equiv), and DMAP (764 mg, $7.42 \mathrm{mmol}, 0.4$ equiv) in $\mathrm{CH}_{2} \mathrm{Cl}_{2}(463 \mathrm{~mL})$ at $0{ }^{\circ} \mathrm{C}$ was added triethylamine $(8.95 \mathrm{~mL}, 55.62$ mmol, 3 equiv) dropwise. The resulting mixture was stirred for 12 hours, gradually warming to room temperature. The reaction was quenched with $400 \mathrm{~mL}$ DI water. It was then extract with $\mathrm{CH}_{2} \mathrm{Cl}_{2}(3 \mathrm{x}$ $200 \mathrm{~mL}$ ), and the combined organic layers were dried over $\mathrm{MgSO}_{4}$, filtered, and concentrated. Purification over silica gel chromatography (38\% EtOAc/Hexanes) afforded 9 (6.99 g, 99\% yield) as a viscous, brown oil (became solid after stored at $4{ }^{\circ} \mathrm{C}$ for several days). $[\alpha]_{\mathrm{D}}^{20}=-16\left(c 1.6, \mathrm{CH}_{2} \mathrm{Cl}_{2}\right)$;

${ }^{1} \mathbf{H}$ NMR $\left(500 \mathrm{MHz} \mathrm{CDCl}_{3}\right): \delta 7.94(\mathrm{~d}, J=3.0 \mathrm{~Hz}, 1 \mathrm{H}), 7.64(\mathrm{~s}, 1 \mathrm{H}), 7.34-7.24(\mathrm{~m}, 5 \mathrm{H}), 6.76(\mathrm{~d}, J=3.0$ $\mathrm{Hz}, 1 \mathrm{H}), 4.55(\mathrm{~d}, J=7.4 \mathrm{~Hz}, 1 \mathrm{H}), 4.45(\mathrm{ABq}, J=12.0 \mathrm{~Hz}, 1 \mathrm{H}), 4.30(\mathrm{ABq}, J=12.0 \mathrm{~Hz}, 1 \mathrm{H}), 3.80(\mathrm{~s}$, $3 \mathrm{H}), 3.68(\mathrm{~s}, 3 \mathrm{H}), 2.94-2.88(\mathrm{~m}, 1 \mathrm{H}), 2.23(\mathrm{~s}, 3 \mathrm{H}), 1.67(\mathrm{~d}, J=2.4 \mathrm{~Hz}, 3 \mathrm{H}), 1.24(\mathrm{~d}, J=6.9 \mathrm{~Hz}, 3 \mathrm{H})$;

${ }^{13} \mathbf{C}$ NMR $\left(125 \mathrm{MHz}, \mathrm{CDCl}_{3}\right): \delta 168.25,156.24,141.35,138.25,133.53,131.63,128.38,128.26$, $127.99,127.78,127.71,127.52,108.11,105.98,80.97,77.79,77.55,70.63,61.66,55.59,32.47,24.96$, 17.81, 3.58;

IR $\left(\right.$ neat $\left./ \mathrm{cm}^{-1}\right) v_{\max }: 3303,2941,1674,1597,1525,1462,1419,1348,1244,1210,1150,1052,997$, 856, 737, 699;

HRMS (ESI) $m / z$ calcd for $\mathrm{C}_{23} \mathrm{H}_{27} \mathrm{NO}_{4}[\mathrm{M}+\mathrm{Na}]^{+} 404.1838$, found 404.1848.
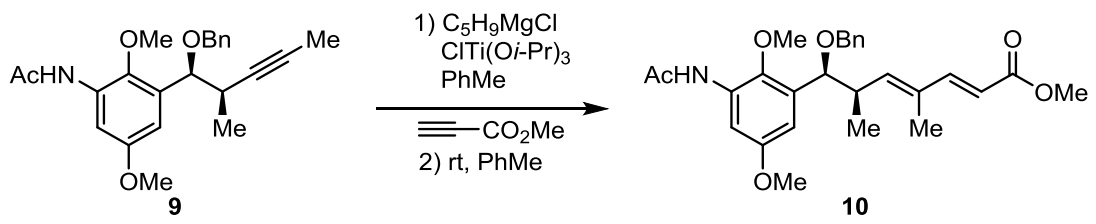

Methyl (2E,4E,6R,7S)-7-(3-acetamido-2,5-dimethoxyphenyl)-7-(benzyloxy)-4,6-dimethylhepta-2,4dienoate (10): To a solution of $9\left(100 \mathrm{mg}, 0.262 \mathrm{mmol}, 1\right.$ equiv) in toluene $(8 \mathrm{~mL})$ at $-78{ }^{\circ} \mathrm{C}$ was added sequentially $\mathrm{ClTi}(\mathrm{O} i \mathrm{Pr})_{3}\left(1.0 \mathrm{M}\right.$ in hexanes, $0.61 \mathrm{~mL}, 0.61 \mathrm{mmol}, 2.3$ equiv) and $c-\mathrm{C}_{5} \mathrm{H}_{9} \mathrm{MgCl}(2.0 \mathrm{M}$ in $\mathrm{Et}_{2} \mathrm{O}, 0.77 \mathrm{~mL}, 1.68 \mathrm{mmol}, 6.4$ equiv) dropwise. The resulting yellow solution turned dark brown while warming slowly to $-40{ }^{\circ} \mathrm{C}$ over $50 \mathrm{~min}$. The reaction mixture was placed in $-40{ }^{\circ} \mathrm{C}$ chiller and stirred for 5 hours, while solution turned to be black and heterogenous. It was then cooled to $-78{ }^{\circ} \mathrm{C}$, to which methyl propiolate ( $48 \mu \mathrm{L}, 0.55 \mathrm{mmol}, 2.1$ equiv) was added dropwise. The resulting mixture was warmed to room temperature over 2 hours and stirred for another 2 hours at room temperature. The reaction was quenched with saturated aqueous $\mathrm{NH}_{4} \mathrm{Cl}(8 \mathrm{~mL})$ solution. It was then extract with EtOAc 
( $3 \times 10 \mathrm{~mL}$ ), and the combined organic layers were dried over $\mathrm{MgSO}_{4}$, filtered, and concentrated. Purification over silica gel chromatography $\left(16 \% \mathrm{EtOAc} / \mathrm{CH}_{2} \mathrm{Cl}_{2}\right.$, then $15 \%$ acetone/Hexanes) afforded 10 $(54 \mathrm{mg}, 45 \%)$ as a colorless, viscous oil and single regioisomer. $[\alpha]_{\mathrm{D}}^{20}=-1.7\left(c 3.3, \mathrm{CH}_{2} \mathrm{Cl}_{2}\right)$;

${ }^{1} \mathbf{H}$ NMR $\left(500 \mathrm{MHz}, \mathrm{CDCl}_{3}\right): \delta 7.87(\mathrm{~d}, J=3.1 \mathrm{~Hz}, 1 \mathrm{H}), 7.57(\mathrm{~s}, 1 \mathrm{H}), 7.34-7.27(\mathrm{~m}, 5 \mathrm{H}), 6.72(\mathrm{~d}, J=3.0$ $\mathrm{Hz}, 1 \mathrm{H}), 5.79(\mathrm{~d}, J=9.9 \mathrm{~Hz}, 1 \mathrm{H}), 5.71(\mathrm{~d}, J=15.6 \mathrm{~Hz}, 1 \mathrm{H}), 4.52(\mathrm{~d}, J=11.8 \mathrm{~Hz}, 1 \mathrm{H}), 4.28(\mathrm{~d}, J=11.8$ $\mathrm{Hz}, 1 \mathrm{H}), 3.78(\mathrm{~s}, 3 \mathrm{H}), 3.73(\mathrm{~s}, 3 \mathrm{H}), 3.61(\mathrm{~s}, 3 \mathrm{H}), 2.94-2.87(\mathrm{~m}, 1 \mathrm{H}), 2.21(\mathrm{~s}, 3 \mathrm{H}), 1.57(\mathrm{~d}, J=1.2 \mathrm{~Hz}$, $3 \mathrm{H}), 1.11(\mathrm{~d}, J=6.7 \mathrm{~Hz}, 3 \mathrm{H})$;

${ }^{13} \mathbf{C}$ NMR $\left(125 \mathrm{MHz}, \mathrm{CDCl}_{3}\right): \delta 168.28,168.00,156.31,149.88,143.93,140.85,138.10,133.78$, $132.34,131.85,128.35,128.31,127.62,115.50,107.84,105.65,78.51,70.92,61.19,55.63,51.44$, 40.08, 24.92, 16.13, 12.06;

IR $\left(\right.$ neat $\left./ \mathrm{cm}^{-1}\right) v_{\max }: 3332,2975,2936,1720,1618,1525,1460,1419,1248,1172,1053$;

HRMS (ESI) $m / z$ calcd for $\mathrm{C}_{27} \mathrm{H}_{33} \mathrm{NO}_{6}[\mathrm{M}+\mathrm{Na}]^{+} 490.2206$, found 490.2213.

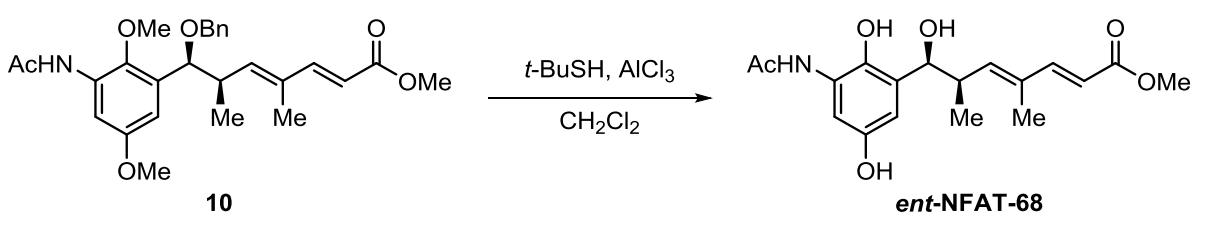

Methyl (2E,4E,6R,7S)-7-(3-acetamido-2,5-dihydroxyphenyl)-7-hydroxy-4,6-dimethylhepta-2,4dienoate (ent-NFAT-68): To a solution of aluminium chloride (114 mg, $0.86 \mathrm{mmol}, 20$ equiv) in dichloromethane $(3 \mathrm{~mL})$ at $0{ }^{\circ} \mathrm{C}$ was added 10 (20 mg, $0.043 \mathrm{mmol}, 1$ equiv) in dichloromethane (1.4 mL) and tert-butylthiol (1.65 mL, $14.55 \mathrm{mmol}, 340$ equiv) sequentially. The resulting solution was stirred at $0{ }^{\circ} \mathrm{C}$ for $30 \mathrm{~min}$. The reaction was quenched with $6 \mathrm{~mL}$ aqueous $\mathrm{NaH}_{2} \mathrm{PO}_{4}$. It was then extract with EtOAc ( $3 \times 4 \mathrm{~mL})$, and the combined organic layers were dried over $\mathrm{MgSO}_{4}$, filtered, and concentrated. Purification over silica gel chromatography $\left(60 \% \mathrm{EtOAc} / \mathrm{CH}_{2} \mathrm{Cl}_{2}\right)$ afforded ent-NFAT-68 $(6 \mathrm{mg}, 41 \%)$ as a colorless, viscous oil. $[\alpha]_{\mathrm{D}}^{20}=+11.3\left(c 0.3, \mathrm{CHCl}_{3}\right)$;

${ }^{1} \mathbf{H}$ NMR (500 MHz, CD $\left.30 D\right): \delta 7.24(\mathrm{dd}, J=15.7,0.6 \mathrm{~Hz}, 1 \mathrm{H}), 6.79(\mathrm{~d}, J=2.9 \mathrm{~Hz}, 1 \mathrm{H}), 6.57(\mathrm{~d}, J=$ $2.7 \mathrm{~Hz}, 1 \mathrm{H}), 5.86(\mathrm{~d}, J=10.0 \mathrm{~Hz}, 1 \mathrm{H}), 5.74(\mathrm{~d}, J=15.4 \mathrm{~Hz}, 1 \mathrm{H}), 4.79(\mathrm{~d}, J=6.3 \mathrm{~Hz}, 1 \mathrm{H}), 3.70(\mathrm{~s}, 3 \mathrm{H})$, $3.01(\mathrm{dq}, J=10.0,6.6 \mathrm{~Hz}, 1 \mathrm{H}), 2.16(\mathrm{~s}, 3 \mathrm{H}), 1.66(\mathrm{~d}, J=1.2 \mathrm{~Hz}, 3 \mathrm{H}), 1.06(\mathrm{~d}, J=6.6 \mathrm{~Hz}, 3 \mathrm{H})$;

${ }^{13}$ C NMR (125 MHz, CD $\left.\mathrm{OD}\right):$ 8171.08, 168.26, 150.04, 149.55, 144.85, 138.97, 132.26, 131.99, $126.39,114.71,111.11,107.92,73.55,50.49,39.42,21.78,14.42,10.92$;

IR $\left(\right.$ neat $\left./ \mathrm{cm}^{-1}\right) v_{\max }: 3346,2935,1694,1622,1543,1459,1376,1317,1198,1017,985,850,757$;

HRMS (ESI) $m / z$ calcd for $\mathrm{C}_{18} \mathrm{H}_{23} \mathrm{NO}_{6}[\mathrm{M}+\mathrm{H}]^{+} 350.1604$, found 350.1602.

\section{b) Total Synthesis of NFAT-68}

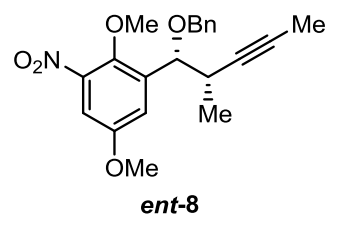

1-((1R,2S)-1-(benzyloxy)-2-methylpent-3-yn-1-yl)-2,5-dimethoxy-3-

nitrobenzene (ent-8): Prepared by the same procedure as 8 . Purification over silica gel chromatography 
$\left(50 \% \mathrm{CH}_{2} \mathrm{Cl}_{2} / \mathrm{Hexanes}\right)$ afforded product ent-8 $(8 \mathrm{~g}, 75 \%$ yield, dr: $5: 1)$ as a yellow oil. $[\alpha]_{\mathrm{D}}^{20}=+4.7(c$ $1.4, \mathrm{CH}_{2} \mathrm{Cl}_{2}$ );

${ }^{1} \mathbf{H}$ NMR $\left(500 \mathrm{MHz} \mathrm{CDCl}_{3}\right): \delta 7.36-7.26(\mathrm{~m}, 7 \mathrm{H}), 4.70(\mathrm{~d}, J=7.3 \mathrm{~Hz}, 1 \mathrm{H}), 4.47(\mathrm{ABq}, J=11.9 \mathrm{~Hz}$, $1 \mathrm{H}), 4.36(\mathrm{ABq}, J=11.9 \mathrm{~Hz}, 1 \mathrm{H}), 3.84(\mathrm{~s}, 3 \mathrm{H}), 3.81(\mathrm{~s}, 3 \mathrm{H}), 2.91-2.84(\mathrm{~m}, 1 \mathrm{H}), 1.68(\mathrm{~d}, J=2.4 \mathrm{~Hz}$, $3 \mathrm{H}), 1.24(\mathrm{~d}, J=6.9 \mathrm{~Hz}, 3 \mathrm{H})$;

${ }^{13}$ C NMR (125 MHz, $\left.\mathrm{CDCl}_{3}\right): \delta 151.64,142.29,139.97,134.62,134.25,124.83,124.25,124.22$, $115.47,106.11,76.77,74.67,73.51,67.65,59.57,52.42,29.37,14.23,0.00$;

IR $\left(\right.$ neat $\left./ \mathrm{cm}^{-1}\right) v_{\max }: 2937,2836,1533,1483,1455,1429,1351,1307,1229,1051,998,737,699$;

HRMS (ESI) $m / z$ calcd for $\mathrm{C}_{21} \mathrm{H}_{23} \mathrm{NO}_{5}[\mathrm{M}+\mathrm{Na}]^{+} 392.1474$, found 392.1467.

Ee analysis of ent-8: Racemic propargylation product and ent-8 were run on chiral HPLC using a ChiralCel AD-H column, isocratic $1 \%$ ispropanol/hexanes at a flow rate of $1 \mathrm{~mL} / \mathrm{min}$. ent-8 was found to have $96 \%$ ee.
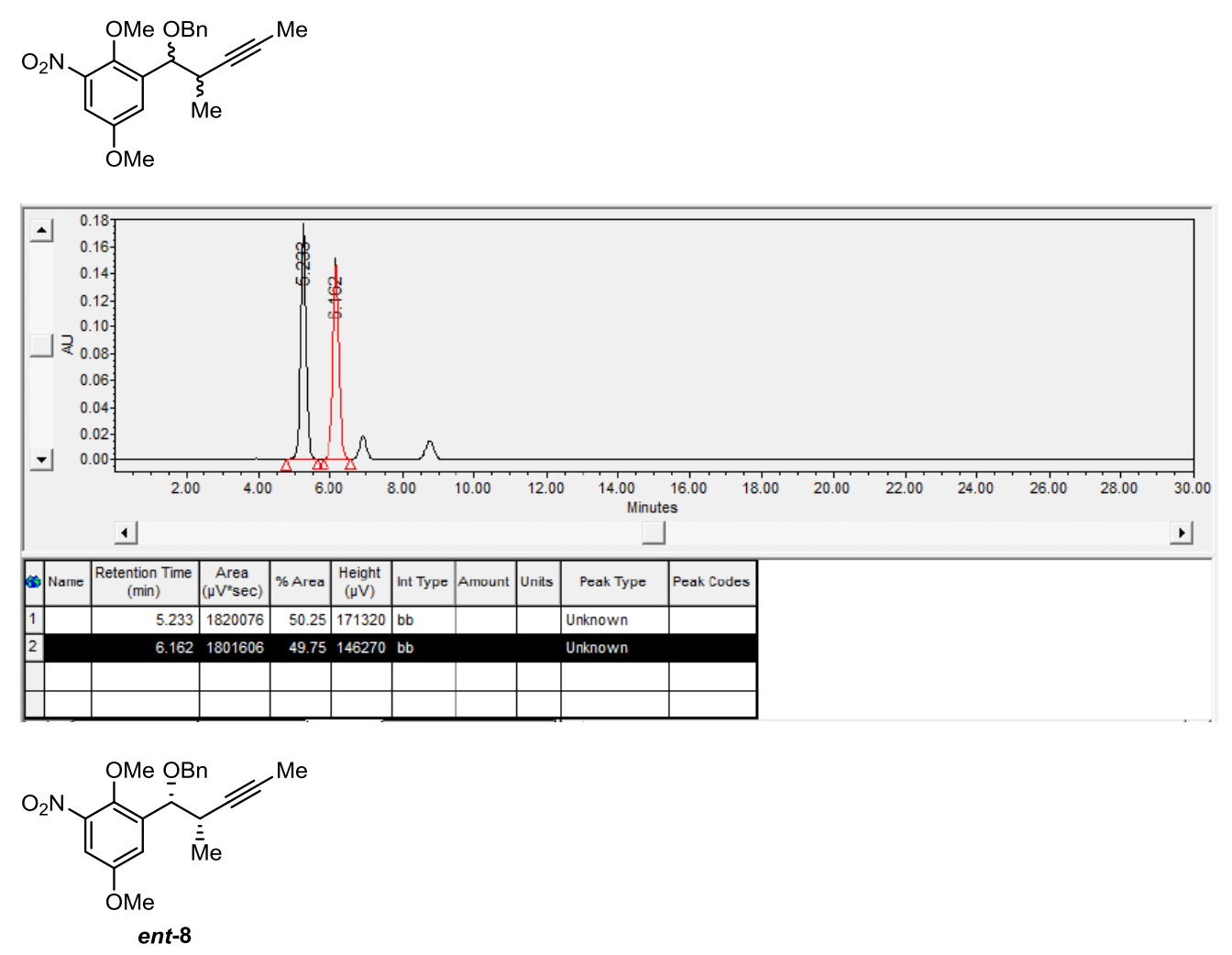

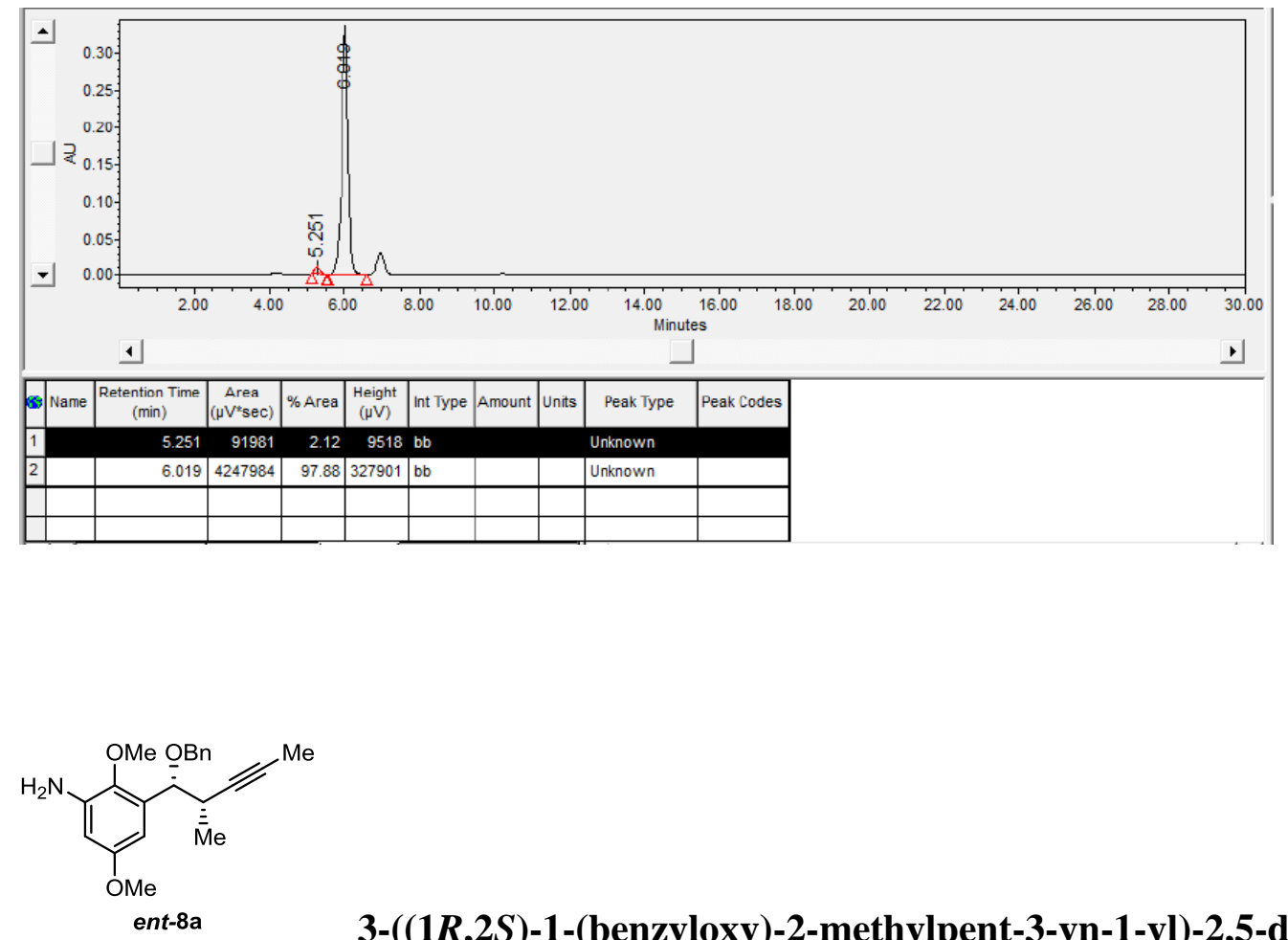

3-((1R,2S)-1-(benzyloxy)-2-methylpent-3-yn-1-yl)-2,5-dimethoxyaniline (ent-

8a): Prepared by the same procedure as 8a. Purification over silica gel chromatography (24\% EtOAc/Hexanes) afforded product ent-8a $(6.13 \mathrm{~g}, 80 \%))$ as a viscous, brown oil. $[\alpha]_{\mathrm{D}}^{20}=+3.7(c 2.7$, $\mathrm{CH}_{2} \mathrm{Cl}_{2}$ );

${ }^{1} \mathbf{H}$ NMR $\left(500 \mathrm{MHz}, \mathrm{CDCl}_{3}\right): \delta 7.35-7.24(\mathrm{~m}, 5 \mathrm{H}), 6.48(\mathrm{~d}, J=3.0 \mathrm{~Hz}, 1 \mathrm{H}), 6.32(\mathrm{~d}, J=3.0 \mathrm{~Hz}, 1 \mathrm{H})$, $4.62(\mathrm{~d}, J=7.1 \mathrm{~Hz}, 1 \mathrm{H}), 4.49(\mathrm{ABq}, J=12.0 \mathrm{~Hz}, 1 \mathrm{H}), 4.34(\mathrm{ABq}, J=12.0 \mathrm{~Hz}, 1 \mathrm{H}), 3.75(\mathrm{~s}, 3 \mathrm{H}), 3.69$ (s, 3H), 2.93-2.86 (m, 1H), $1.70(\mathrm{dd}, J=2.4,0.5 \mathrm{~Hz}, 3 \mathrm{H}), 1.24(\mathrm{~d}, J=6.9 \mathrm{~Hz}, 3 \mathrm{H})$;

${ }^{13}$ C NMR (125 MHz, $\left.\mathrm{CDCl}_{3}\right): \delta 156.40,140.67,139.45,138.60,134.07,128.19,127.71,127.38$, $102.23,102.01,81.37,77.87,77.17,70.67,60.28,55.37,32.56,17.58,3.18$;

IR $\left(\right.$ neat $\left./ \mathrm{cm}^{-1}\right) v_{\max }: 3457,3367,2938,1614,1495,1454,1349,1240,1197,1170,1045,1001,838$, 736, 698;

HRMS (ESI) $m / z$ calcd for $\mathrm{C}_{21} \mathrm{H}_{25} \mathrm{NO}_{3}[\mathrm{M}+\mathrm{H}]^{+} 340.1913$, found 340.1904.

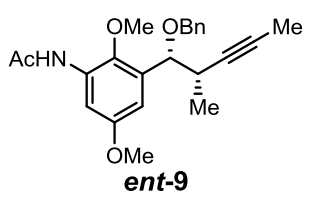

$N-(3-((1 R, 2 S)-1-(b e n z y l o x y)-2-m e t h y l p e n t-3-y n-1-y l)-2,5-$

dimethoxyphenyl)acetamide (ent-9): Prepared by the same procedure as $\mathbf{9}$. Purification over silica gel chromatography (38\% EtOAc/Hexanes) afforded product $\boldsymbol{e n t} \mathbf{- 9}$ (6.87 g, 99\% yield) as a viscous, brown oil (became solid after stored at $4{ }^{\circ} \mathrm{C}$ for several days). $[\alpha]_{\mathrm{D}}^{20}=+2.3\left(c 5.1, \mathrm{CH}_{2} \mathrm{Cl}_{2}\right)$;

${ }^{1} \mathbf{H}$ NMR $\left(500 \mathrm{MHz}, \mathrm{CDCl}_{3}\right): 7.93(\mathrm{~d}, J=2.9 \mathrm{~Hz}, 1 \mathrm{H}), 7.67(\mathrm{~s}, 1 \mathrm{H}), \delta 7.36-7.24(\mathrm{~m}, 5 \mathrm{H}), 6.76(\mathrm{~d}, J=3.0$ $\mathrm{Hz}, 1 \mathrm{H}), 4.56(\mathrm{~d}, J=7.4 \mathrm{~Hz}, 1 \mathrm{H}), 4.45(\mathrm{ABq}, J=12.0 \mathrm{~Hz}, 1 \mathrm{H}), 4.30(\mathrm{ABq}, J=12.0 \mathrm{~Hz}, 1 \mathrm{H}), 3.79(\mathrm{~s}$, $3 \mathrm{H}), 3.68(\mathrm{~s}, 3 \mathrm{H}), 2.94-2.87(\mathrm{~m}, 1 \mathrm{H}), 2.22(\mathrm{~s}, 3 \mathrm{H}), 1.66(\mathrm{~d}, J=2.4 \mathrm{~Hz}, 3 \mathrm{H}), 1.24(\mathrm{~d}, J=6.9 \mathrm{~Hz}, 3 \mathrm{H})$;

${ }^{13} \mathrm{C}$ NMR (125 MHz, $\left.\mathrm{CDCl}_{3}\right): \delta 168.27,156.24,141.38,138.26,133.54,131.63,128.24,127.70$, 127.51, 108.13, 106.04, 80.98, 77.82, 77.53, 70.65, 61.64, 55.58, 32.47, 24.92, 17.79, 3.56; 
IR $\left(\right.$ neat $\left./ \mathrm{cm}^{-1}\right) v_{\max }: 3278,2939,1675,1595,1524,1463,1419,1348,1244,1209,1150,1053,997$, 856, 737, 698;

HRMS (ESI) $m / z$ calcd for $\mathrm{C}_{23} \mathrm{H}_{27} \mathrm{NO}_{4}[\mathrm{M}+\mathrm{Na}]^{+} 404.1838$, found 404.1836.

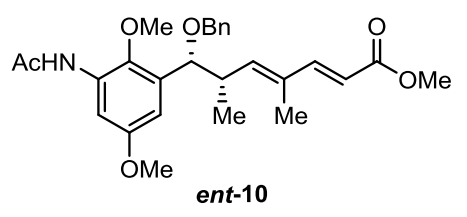

Methyl (2E,4E,6S,7R)-7-(3-acetamido-2,5-dimethoxyphenyl)-7(benzyloxy)-4,6-dimethylhepta-2,4-dienoate (ent-10): Prepared by the same procedure as 10. Purification over silica gel chromatography $\left(16 \% \mathrm{EtOAc} / \mathrm{CH}_{2} \mathrm{Cl}_{2}\right.$, then $15 \%$ acetone/Hexanes) afforded product ent-10 (53 mg, 44\% yield) as a viscous, colorless oil and single regioisomer. $[\alpha]_{\mathrm{D}}^{20}=-1.7(c 3.3$, $\mathrm{CH}_{2} \mathrm{Cl}_{2}$ );

${ }^{1} \mathbf{H}$ NMR $\left(500 \mathrm{MHz}, \mathrm{CDCl}_{3}\right): \delta 7.87(\mathrm{~d}, J=3.1 \mathrm{~Hz}, 1 \mathrm{H}), 7.56(\mathrm{~s}, 1 \mathrm{H}), 7.25-7.28(\mathrm{~m}, 5 \mathrm{H}), 7.22(\mathrm{~d}, J=$ $15.7 \mathrm{~Hz}, 1 \mathrm{H}), 5.79(\mathrm{~d}, J=9.9 \mathrm{~Hz}, 1 \mathrm{H}), 5.71(\mathrm{~d}, J=15.7 \mathrm{~Hz}, 1 \mathrm{H}), 4.52(\mathrm{ABq}, J=11.7 \mathrm{~Hz}, 1 \mathrm{H}), 4.50(\mathrm{~d}$, $J=6.7 \mathrm{~Hz}, 1 \mathrm{H}), 4.28(\mathrm{ABq}, J=11.8 \mathrm{~Hz}, 1 \mathrm{H}), 3.78(\mathrm{~s}, 3 \mathrm{H}), 3.73(\mathrm{~s}, 3 \mathrm{H}), 3.61(\mathrm{~s}, 3 \mathrm{H}), 2.94-2.87(\mathrm{~m}$, $1 \mathrm{H}), 2.22(\mathrm{~s}, 3 \mathrm{H}), 1.57(\mathrm{~d}, J=1.1 \mathrm{~Hz}, 3 \mathrm{H}), 1.11(\mathrm{~d}, J=6.7 \mathrm{~Hz}, 3 \mathrm{H})$;

${ }^{13}$ C NMR $\left(125 \mathrm{MHz}, \mathrm{CDCl}_{3}\right): \delta 168.26,168.00,156.32,149.88,143.93,140.85,138.10,133.78$, $132.34,131.84,128.35,128.31,127.62,115.50,107.85,105.65,78.51,70.92,61.20,55.63,51.45$, 40.08, 24.93, 16.14, 12.06;

IR $\left(\right.$ neat $\left./ \mathrm{cm}^{-1}\right) v_{\max }: 3332,2941,1695,1621,1526,1459,1418,1170,997,848,738,699$;

HRMS (ESI) $m / z$ calcd for $\mathrm{C}_{27} \mathrm{H}_{33} \mathrm{NO}_{6}[\mathrm{M}+\mathrm{H}]^{+} 468.2386$, found 468.2379.

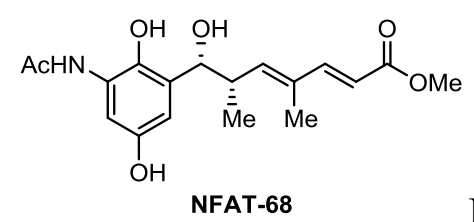

dimethylhepta-2 4-dienoate (NFAT-68): Prepared by the same procedure as ent NFAT-68. Purification over silica gel chromatography $\left(60 \% \mathrm{EtOAc} / \mathrm{CH}_{2} \mathrm{Cl}_{2}\right)$ afforded product NFAT-68 $(4 \mathrm{mg}, 41 \%$ yield $)$ as a viscous, colorless oil. $[\alpha]_{\mathrm{D}}^{20}=-11.4\left(c 0.3, \mathrm{CHCl}_{3}\right)$;

${ }^{1} \mathbf{H}$ NMR $\left(500 \mathrm{MHz}, \mathrm{CD}_{3} \mathrm{OD}\right): \delta 7.24(\mathrm{dd}, J=15.7,0.6 \mathrm{~Hz}, 1 \mathrm{H}), 6.79(\mathrm{~d}, J=2.9 \mathrm{~Hz}, 1 \mathrm{H}), 6.57(\mathrm{~d}, J=$ $2.9 \mathrm{~Hz}, 1 \mathrm{H}), 5.86(\mathrm{~d}, J=9.9 \mathrm{~Hz}, 1 \mathrm{H}), 5.74(\mathrm{~d}, J=15.4 \mathrm{~Hz}, 1 \mathrm{H}), 4.79(\mathrm{~d}, J=6.3 \mathrm{~Hz}, 1 \mathrm{H}), 3.70(\mathrm{~s}, 3 \mathrm{H})$, $3.01(\mathrm{dq}, J=10.1,6.6 \mathrm{~Hz}, 1 \mathrm{H}), 2.16(\mathrm{~s}, 3 \mathrm{H}), 1.66(\mathrm{~d}, J=1.2 \mathrm{~Hz}, 3 \mathrm{H}), 1.06(\mathrm{~d}, J=6.7 \mathrm{~Hz}, 3 \mathrm{H})$;

${ }^{13}$ C NMR (125 MHz, CD $\left.3 \mathrm{OD}\right)$ : $\delta 171.06,168.26,150.04,149.55,144.85,138.97,132.26,131.99$, $126.39,114.71,111.11,107.92,73.55,50.49,39.42,21.78,14.42,10.92$;

IR $\left(\right.$ neat $\left./ \mathrm{cm}^{-1}\right) v_{\max }: 3342,1693,1623,1547,1460,1375,1317,1202,984,852,760 ;$

HRMS (ESI) $m / z$ calcd for $\mathrm{C}_{18} \mathrm{H}_{23} \mathrm{NO}_{6}[\mathrm{M}+\mathrm{H}]^{+} 350.1604$, found 350.1604. 


\section{viii. NFAT-68 Spectral Data Comparison ${ }^{6}$}

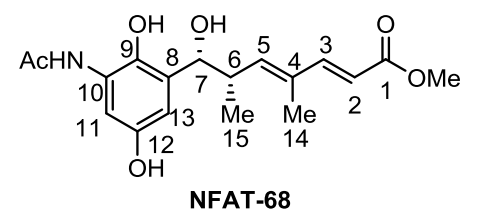

\begin{tabular}{|l|l|l|c|}
\hline \multirow{2}{*}{ Position } & \multicolumn{3}{|c|}{${ }^{1} \mathrm{H}$ NMR (mult, $J$ in Hz, CD 3 OD) } \\
\cline { 2 - 4 } & \multicolumn{1}{|c|}{$\begin{array}{c}\text { Panek } \delta \text { Cai } \\
(500 \mathrm{MHz}, \text { ref. 3.30ppm) }\end{array}$} & \multicolumn{1}{c|}{ Mcalpine } & $\Delta \delta(\mathrm{ppm})$ \\
\hline $1-\mathrm{OCH}_{3}$ & $3.70(\mathrm{~s}, 3 \mathrm{H})$ & $3.70(\mathrm{~s}, 3 \mathrm{H})$ & 0 \\
\hline 2 & $5.74(\mathrm{~d}, J=15.4 \mathrm{~Hz}, 1 \mathrm{H})$ & $5.72(\mathrm{~d}, 1 \mathrm{H}, J=15.7 \mathrm{~Hz})$ & +0.02 \\
\hline 3 & $7.24(\mathrm{dd}, J=15.7,0.6 \mathrm{~Hz}, 1 \mathrm{H})$ & $7.22(\mathrm{br} \mathrm{d}, 1 \mathrm{H}, J=15.7 \mathrm{~Hz})$ & +0.02 \\
\hline 5 & $5.86(\mathrm{~d}, J=9.9 \mathrm{~Hz}, 1 \mathrm{H})$ & $5.84(\mathrm{brd}, 1 \mathrm{H}, J=9.9 \mathrm{~Hz})$ & +0.02 \\
\hline 6 & $3.01(\mathrm{dq}, J=10.1,6.6 \mathrm{~Hz}, 1 \mathrm{H})$ & $3.01(\mathrm{mult}, 1 \mathrm{H})$ & 0 \\
\hline 7 & $4.79(\mathrm{~d}, J=6.3 \mathrm{~Hz}, 1 \mathrm{H})$ & $4.80(\mathrm{~d}, 1 \mathrm{H}, J=7.2 \mathrm{~Hz})$ & +0.01 \\
\hline $10-\mathrm{NAc}$ & $2.16(\mathrm{~s}, 3 \mathrm{H})$ & $2.15(\mathrm{~s}, 3 \mathrm{H})$ & -0.01 \\
\hline 11 & $6.79(\mathrm{~d}, J=2.9 \mathrm{~Hz}, 1 \mathrm{H})$ & $6.80(\mathrm{~d}, 1 \mathrm{H}, J=2.9 \mathrm{~Hz})$ & 0 \\
\hline 13 & $6.57(\mathrm{~d}, J=2.9 \mathrm{~Hz}, 1 \mathrm{H})$ & $6.57(\mathrm{~d}, 1 \mathrm{H}, J=2.9 \mathrm{~Hz})$ & +0.03 \\
\hline 14 & $1.66(\mathrm{~d}, J=1.2 \mathrm{~Hz}, 3 \mathrm{H})$ & $1.63(\mathrm{br}, 3 \mathrm{H})$ & +0.04 \\
\hline 15 & $1.06(\mathrm{~d}, J=6.7 \mathrm{~Hz}, 3 \mathrm{H})$ & $1.02(\mathrm{~d}, 3 \mathrm{H}, J=6.6 \mathrm{~Hz})$ & \\
\hline
\end{tabular}

\begin{tabular}{|l|c|c|c|}
\hline \multirow{2}{*}{ Position } & \multicolumn{3}{|c|}{${ }^{13} \mathrm{C} \mathrm{NMR}\left(\mathrm{CD}_{3} \mathrm{OD}\right)$} \\
\cline { 2 - 4 } & $\begin{array}{c}\text { Panek } \delta \text { Cai } \\
(125 \mathrm{MHz}, \text { ref. 47.58 ppm) }\end{array}$ & Mcalpine & $\Delta \delta(\mathrm{ppm})$ \\
\hline 1 & 168.26 & 169.7 & -1.44 \\
\hline $1-\mathrm{OCH}_{3}$ & 50.49 & 51.9 & -1.41 \\
\hline 2 & 144.85 & 146.2 & -1.35 \\
\hline 3 & 150.04 & 151.4 & -1.36 \\
\hline 4 & 132.26 & 133.7 & -1.44 \\
\hline 5 & 114.71 & 116.1 & -1.39 \\
\hline 6 & 39.42 & 40.8 & -1.38 \\
\hline 7 & 73.55 & 75.1 & -1.55 \\
\hline 8 & 131.99 & 133.2 & -1.21 \\
\hline 9 & 138.97 & 140.3 & -1.33 \\
\hline 10 & 126.39 & 127.8 & -1.41 \\
\hline $10-\mathrm{NAc}$ & 171.06 & 172.5 & -1.44 \\
\hline & 21.78 & 23.3 & -1.52 \\
\hline 11 & 107.92 & 109.3 & -1.38 \\
\hline 12 & 149.55 & 150.9 & -1.35 \\
\hline 13 & 111.11 & 112.5 & -1.39 \\
\hline 14 & 14.42 & 15.9 & -1.48 \\
\hline 15 & 10.92 & 12.4 & -1.48 \\
\hline
\end{tabular}

${ }^{6}$ Burres, N. S.; Premachandran, U.; Hoselton, S.; Cwik, D.; Hochlowski, J. E.; Ye, Q. M.; Sunga, G. N.; Karwowski, J. P.; Jackson, M.; Whittern, D. N.; Mcalpine, J. B. J. Antibiot. 1995, 48, 380. 
ix. Confirmation of Relative Stereochemistry of ent-8 and Absolute Stereochemistry of 9 and Ent-9

a) Confirmation of Relative Stereochemistry of ent-8 by Converting ent-8 to Acetonide 15 and Measuring Three-Bond Coupling Constants.
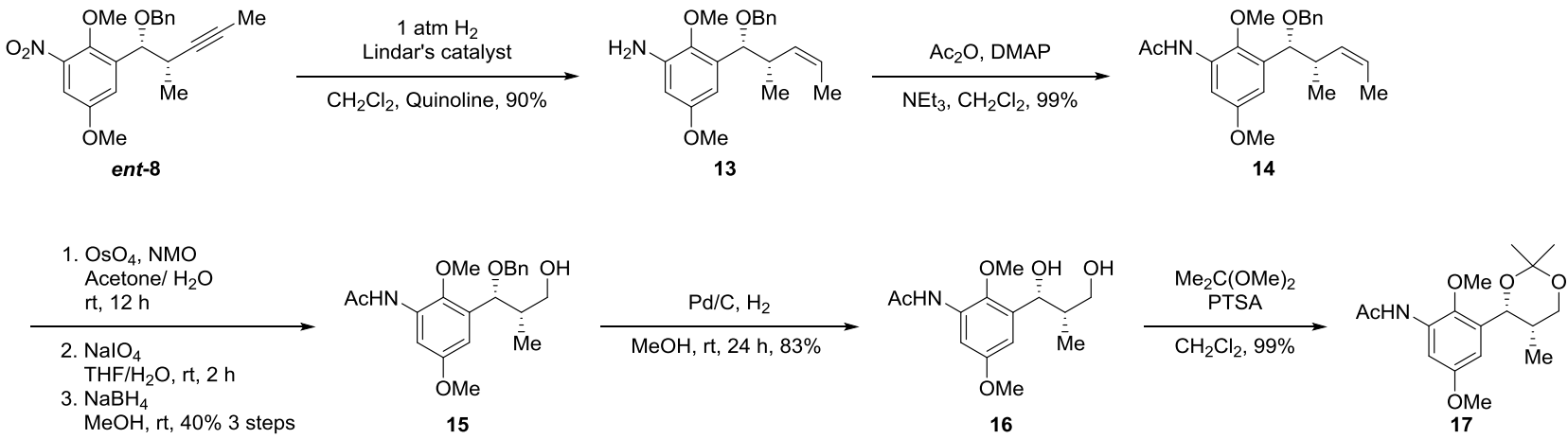

Ent-8 was reduced to Z-alkene 13 by hydrogenation using Lindar's catalyst, while the arylnitro group was simultaneously reduced to amino group. Subsequent $N$-acylation of $\mathbf{1 3}$ produced arylacetamide 14. A three-step sequence involving dihydroxylation, oxidation, and reduction obtained primary alcohol 15. Deprotection of benzyl ether of $\mathbf{1 7}$ was achieved by hydrogenation to give diol 16. Lastly, acetonide 17 was formed by acid-catalyzed ketalization. Three-bond coupling constant between $\mathrm{H}_{1}$ and $\mathrm{H}_{2}$ is $2.5 \mathrm{~Hz}$, indicating the syn relationship between $\mathrm{C} 1$ methyl group and $\mathrm{C} 2$ oxygen.
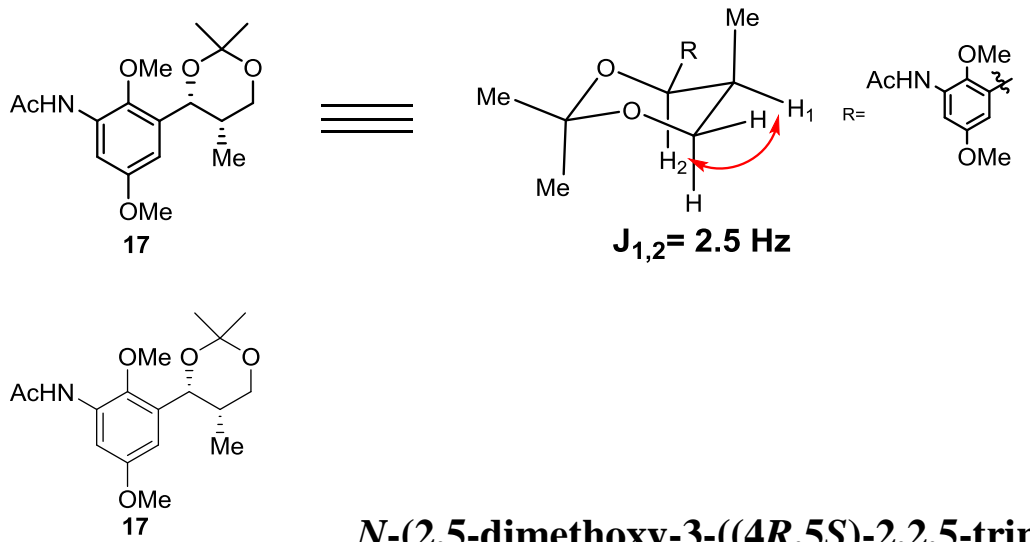

$N$-(2,5-dimethoxy-3-((4R,5S)-2,2,5-trimethyl-1,3-dioxan-4-yl)phenyl)acetamide (17): 17 was isolated as a light brown solid. $[\alpha]_{\mathrm{D}}^{20}=+2.4\left(c 1.9, \mathrm{CH}_{2} \mathrm{Cl}_{2}\right)$;

${ }^{1}$ H NMR (500 MHz, $\left.\mathrm{CDCl}_{3}\right): \delta 7.87(\mathrm{~s}, 1 \mathrm{H}), 7.55(\mathrm{~s}, 1 \mathrm{H}), 6.76(\mathrm{~s}, 1 \mathrm{H}), 5.32(\mathrm{~d}, J=2.2 \mathrm{~Hz}, 1 \mathrm{H}), 4.30$ $(\mathrm{dd}, J=11.5,2.4 \mathrm{~Hz}, 1 \mathrm{H}), 3.79(\mathrm{~s}, 3 \mathrm{H}), 3.72(\mathrm{~s}, 3 \mathrm{H}), 3.66(\mathrm{~d}, J=11.5 \mathrm{~Hz}, 1 \mathrm{H}), 2.21(\mathrm{~s}, 3 \mathrm{H}), 1.90-1.82$ $(\mathrm{m}, 1 \mathrm{H}), 1.56(\mathrm{~s}, 3 \mathrm{H}), 1.51(\mathrm{~s}, 3 \mathrm{H}), 0.83(\mathrm{~d}, J=7.0 \mathrm{~Hz}, 3 \mathrm{H})$;

${ }^{13}$ C NMR (125 MHz, $\left.\mathrm{CDCl}_{3}\right): \delta 168.133,156.08,138.89,134.17,131.52,108.82,104.61,99.10,68.77$, $66.36,61.03,55.59,32.55,29.64,18.98,11.43$;

IR $\left(\right.$ neat $\left./ \mathrm{cm}^{-1}\right) v_{\max }: 3263,3006,2945,1664,1619,1594,1541,1461,1421,1379,1358,1254,1225$, 1243, 1194, 1172, 1150, 1135, 1108, 1059, 1002, 877, 865, 845, 800, 760, 744, 707;

HRMS (ESI) $m / z$ calcd for $\mathrm{C}_{17} \mathrm{H}_{25} \mathrm{NO}_{5}[\mathrm{M}+\mathrm{Na}]^{+} 346.1630$, found 346.1615 .

\section{b) Confirmation of Absolute Stereochemistry of 9 and ent-9}




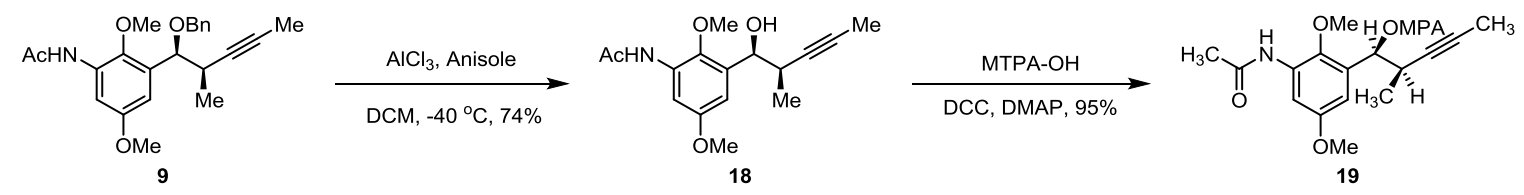

The absolute stereochemistry of 9 and ent-9 was determined by converting them to Mosher's esters ${ }^{7} 19$ and ent-19.

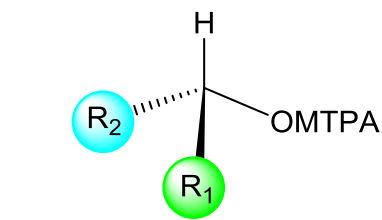

${ }^{*}$ For protons $\Delta \delta^{S R}>0$, reside within $R_{1}$; For protons $\Delta \delta \delta^{S R}<0$, reside within $R_{2}$.

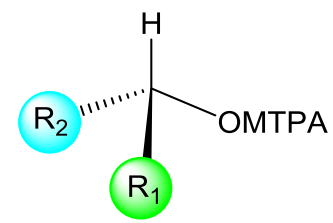

${ }^{*}$ For protons $\Delta \delta \delta^{S R}>0$, reside within $R_{1}$; For protons $\Delta \delta R^{S \text {, reside within }} \mathrm{R}_{2}$.

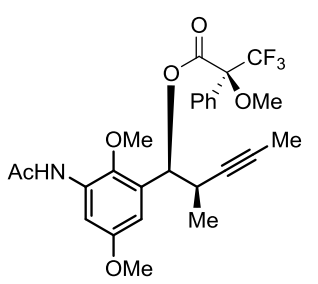

(S)-19<smiles>CC#C[C@H](C)[C@@H](C)c1cc(OC)cc(NC(C)=O)c1O[Na]</smiles>

19

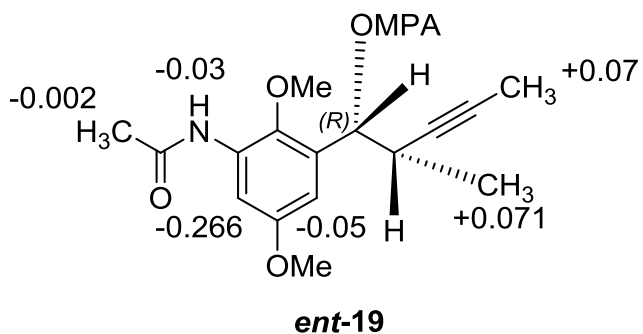

trifluoro-2-methoxy-2-phenylpropanoate $((S)-19):(S)-19$ was obtained as a colorless, viscous oil. $[\alpha]_{\mathrm{D}}^{20}=-3.8\left(c 1.5, \mathrm{CH}_{2} \mathrm{Cl}_{2}\right)$;

${ }^{1} \mathbf{H}$ NMR $\left(500 \mathrm{MHz}, \mathrm{CDCl}_{3}\right): \delta 7.96(\mathrm{~d}, J=2.8 \mathrm{~Hz}, 1 \mathrm{H}), 7.66(\mathrm{~s}, 1 \mathrm{H}), 7.44-7.26(\mathrm{~m}, 5 \mathrm{H}), 6.58(\mathrm{~d}, J=2.9$ $\mathrm{Hz}, 1 \mathrm{H}), 6.19(\mathrm{~d}, J=7.1 \mathrm{~Hz}, 1 \mathrm{H}), 3.86(\mathrm{~s}, 3 \mathrm{H}), 3.73(\mathrm{~s}, 3 \mathrm{H}), 3.47(\mathrm{~s}, 3 \mathrm{H}), 3.00-2.93(\mathrm{~m}, 1 \mathrm{H}), 2.22(\mathrm{~s}$, $3 \mathrm{H}), 1.65(\mathrm{~d}, J=2.4 \mathrm{~Hz}, 3 \mathrm{H}), 1.07(\mathrm{~d}, J=6.9 \mathrm{~Hz}, 3 \mathrm{H})$;

${ }^{13} \mathbf{C}$ NMR $\left(100 \mathrm{MHz}, \mathrm{CDCl}_{3}\right): \delta 165.02,162.50,152.75,136.98,128.64,127.40,126.47,126.09$, $125.19,124.79,124.38,124.02,121.41,118.54,104.26,103.36,81.38,81.10,75.66,75.10,71.44$, 71.18, 58.47, 58.23, 52.30, 52.04, 28.37, 28.05, 21.65, 21.54, 13.55, 13.37, 0.20, 0.00;

IR (neat $/ \mathrm{cm}^{-1}$ ) $v_{\max }: 3305,2988,2945,2844,1751,1681,1599,1529,1466,1422,1367,1248,1172$, 1123, 1054, 1019, 996, 723;

HRMS (ESI) $m / z$ calcd for $\mathrm{C}_{26} \mathrm{H}_{28} \mathrm{~F}_{3} \mathrm{NO}_{6}[\mathrm{M}+\mathrm{Na}]^{+} 530.1766$, found 530.1782 .

${ }^{7}$ Hoye, T. R.; Jeffrey, C. S.; Shao, F. Nat. Protoc. 2007, 2, 2451. 


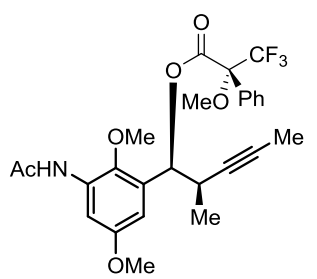

$(R)-19 \quad(1 S, 2 R)-1-(3-a c e t a m i d o-2,5-d i m e t h o x y p h e n y l)-2-m e t h y l p e n t-3-y n-1-y l ~(R)-3,3,3-$ trifluoro-2-methoxy-2-phenylpropanoate $((\boldsymbol{R})-19):(R)-19$ was obtained as a colorless, viscous oil. $[\alpha]_{\mathrm{D}}^{20}=+2.0\left(c 2.1, \mathrm{CH}_{2} \mathrm{Cl}_{2}\right)$;

${ }^{1} \mathbf{H}$ NMR $\left(500 \mathrm{MHz}, \mathrm{CDCl}_{3}\right): \delta 7.91(\mathrm{~d}, J=2.8 \mathrm{~Hz}, 1 \mathrm{H}), 7.64(\mathrm{~s}, 1 \mathrm{H}), 7.48-7.32(\mathrm{~m}, 5 \mathrm{H}), 6.31(\mathrm{~d}, J=2.9$ $\mathrm{Hz}, 1 \mathrm{H}), 6.20(\mathrm{~d}, J=5.6 \mathrm{~Hz}, 1 \mathrm{H}), 3.87(\mathrm{~s}, 3 \mathrm{H}), 3.61(\mathrm{~s}, 3 \mathrm{H}), 3.60(\mathrm{~d}, J=1.1 \mathrm{~Hz}, 3 \mathrm{H}), 2.99-2.92(\mathrm{~m}$, $1 \mathrm{H}), 2.21(\mathrm{~s}, 3 \mathrm{H}), 1.72(\mathrm{~d}, J=2.4 \mathrm{~Hz}, 3 \mathrm{H}), 1.14(\mathrm{~d}, J=7.0 \mathrm{~Hz}, 3 \mathrm{H})$;

${ }^{13}$ C NMR (125 MHz, $\left.\mathrm{CDCl}_{3}\right): \delta 168.27,165.73,155.93,139.97,132.03,131.89,130.48,129.54$, $128.29,127.68,127.32,124.46,122.17,107.12$, 106.87, 79.57, 78.35, 74.97, 61.42, 55.75, 55.34, 31.41, $24.89,16.16,3.46$

IR (neat/cm $\left.{ }^{-1}\right) v_{\max }: 3334,2943,2848,1750,1681,1602,1527,1465,1421,1366,1247,1168,1124$, 1081, 1054, 1018, 993, 723, 698;

HRMS (ESI) $m / z$, calcd for $\mathrm{C}_{26} \mathrm{H}_{28} \mathrm{~F}_{3} \mathrm{NO}_{6}[\mathrm{M}+\mathrm{Na}]^{+} 530.1766$, found 530.1747 .

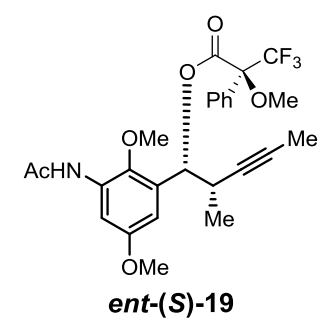

$(1 R, 2 S)-1-(3-a c e t a m i d o-2,5-d i m e t h o x y p h e n y l)-2-m e t h y l p e n t-3-y n-1-y l \quad(S)-3,3,3-$ trifluoro-2-methoxy-2-phenylpropanoate $($ ent-(S)-19): Ent-(S)-19 was obtained as a colorless, viscous oil. $[\alpha]_{\mathrm{D}}^{20}=-8.6\left(c 4.1, \mathrm{CH}_{2} \mathrm{Cl}_{2}\right)$;

${ }^{1} \mathbf{H}$ NMR $\left(500 \mathrm{MHz}, \mathrm{CDCl}_{3}\right): \delta 7.92(\mathrm{~d}, J=2.7 \mathrm{~Hz}, 1 \mathrm{H}), 7.61(\mathrm{~s}, 1 \mathrm{H}), 7.48-7.33(\mathrm{~m}, 5 \mathrm{H}), 6.31(\mathrm{~d}, J=2.7$ $\mathrm{Hz}, 1 \mathrm{H}), 6.19(\mathrm{~d}, J=5.6 \mathrm{~Hz}, 1 \mathrm{H}), 3.87(\mathrm{~s}, 3 \mathrm{H}), 3.61(\mathrm{~s}, 3 \mathrm{H}), 3.60(\mathrm{~s}, 3 \mathrm{H}), 2.99-2.92(\mathrm{~m}, 1 \mathrm{H}), 2.22(\mathrm{~s}$, $3 \mathrm{H}), 1.73(\mathrm{~d}, J=2.3 \mathrm{~Hz}, 3 \mathrm{H}), 1.15(\mathrm{~d}, J=7.0 \mathrm{~Hz}, 3 \mathrm{H})$;

${ }^{13} \mathrm{C}$ NMR (125 MHz, $\left.\mathrm{CDCl}_{3}\right): \delta 168.26,165.74,155.93,139.93,132.02,131.88,130.48,129.55$, $128.30,127.32,124.43,122.15,107.09,106.81,79.57,78.35,74.98,61.44,55.76,55.35,31.40,24.93$, $16.18,3.49$;

IR $\left(\right.$ neat $\left./ \mathrm{cm}^{-1}\right) v_{\max }: 3321,2988,2949,2844,1751,1680,1602,1527,1466,1421,1369,1247,1170$, 1124, 1054, 1019, 994, 723;

HRMS (ESI) $m / z$ calcd for $\mathrm{C}_{26} \mathrm{H}_{28} \mathrm{~F}_{3} \mathrm{NO}_{6}[\mathrm{M}+\mathrm{H}]^{+} 508.1947$, found 508.1930. 


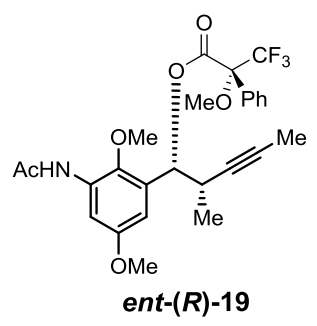

(1R,2S)-1-(3-acetamido-2,5-dimethoxyphenyl)-2-methylpent-3-yn-1-yl $(R)-3,3,3-$ trifluoro-2-methoxy-2-phenylpropanoate $($ ent-(R)-19): $\boldsymbol{E n t}$ - $(\boldsymbol{R})-19$ was obtained as a colorless, viscous oil. $[\alpha]_{\mathrm{D}}^{20}=+3.4\left(c 4.8, \mathrm{CH}_{2} \mathrm{Cl}_{2}\right)$;

${ }^{1}$ H NMR $\left(500 \mathrm{MHz}, \mathrm{CDCl}_{3}\right): \delta 7.97(\mathrm{~d}, J=2.8 \mathrm{~Hz}, 1 \mathrm{H}), 7.65(\mathrm{~s}, 1 \mathrm{H}), 7.44-7.29(\mathrm{~m}, 1 \mathrm{H}), 6.57(\mathrm{~d}, J=2.8$ $\mathrm{Hz}, 1 \mathrm{H}), 6.19(\mathrm{~d}, J=7.1 \mathrm{~Hz}, 1 \mathrm{H}), 3.87(\mathrm{~s}, 3 \mathrm{H}), 3.73(\mathrm{~s}, 3 \mathrm{H}), 3.47(\mathrm{~s}, 3 \mathrm{H}), 2.99-2.93(\mathrm{~m}, 1 \mathrm{H}), 2.22(\mathrm{~s}$, $3 \mathrm{H}), 1.65(\mathrm{~d}, J=2.3 \mathrm{~Hz}, 1 \mathrm{H}), 1.07(\mathrm{~d}, J=6.9 \mathrm{~Hz}, 3 \mathrm{H})$;

${ }^{13}$ C NMR $\left(125 \mathrm{MHz}, \mathrm{CDCl}_{3}\right): \delta 168.33,165.82,156.09,140.31,131.98,130.73,129.61,129.49$, $128.33,128.23,127.54,127.26,124.46,122.17,107.65,106.69,78.99,78.44,74.65,61.68,55.52$, $55.42,31.55,24.93,16.79,3.43$;

IR $\left(\right.$ neat $\left./ \mathrm{cm}^{-1}\right) v_{\max }: 3320,2987,2948,2845,1749,1677,1601,1526,1465,1421,1366,1246,1170$, 1122, 1081, 1054, 1017, 995, 915, 734, 698;

HRMS (ESI) $m / z$ calcd for $\mathrm{C}_{26} \mathrm{H}_{28} \mathrm{~F}_{3} \mathrm{NO}_{6}[\mathrm{M}+\mathrm{Na}]^{+} 530.1766$, found 530.1758 . 


\section{Part III: ${ }^{1} \mathrm{H}$ - and ${ }^{13} \mathrm{C}$-NMR Spectra:}

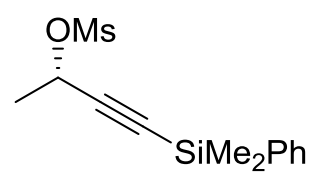

(S)-12

$\mathrm{CDCl}_{3}$

$500 \mathrm{MHz}^{1} \mathrm{H}$

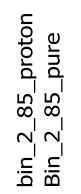

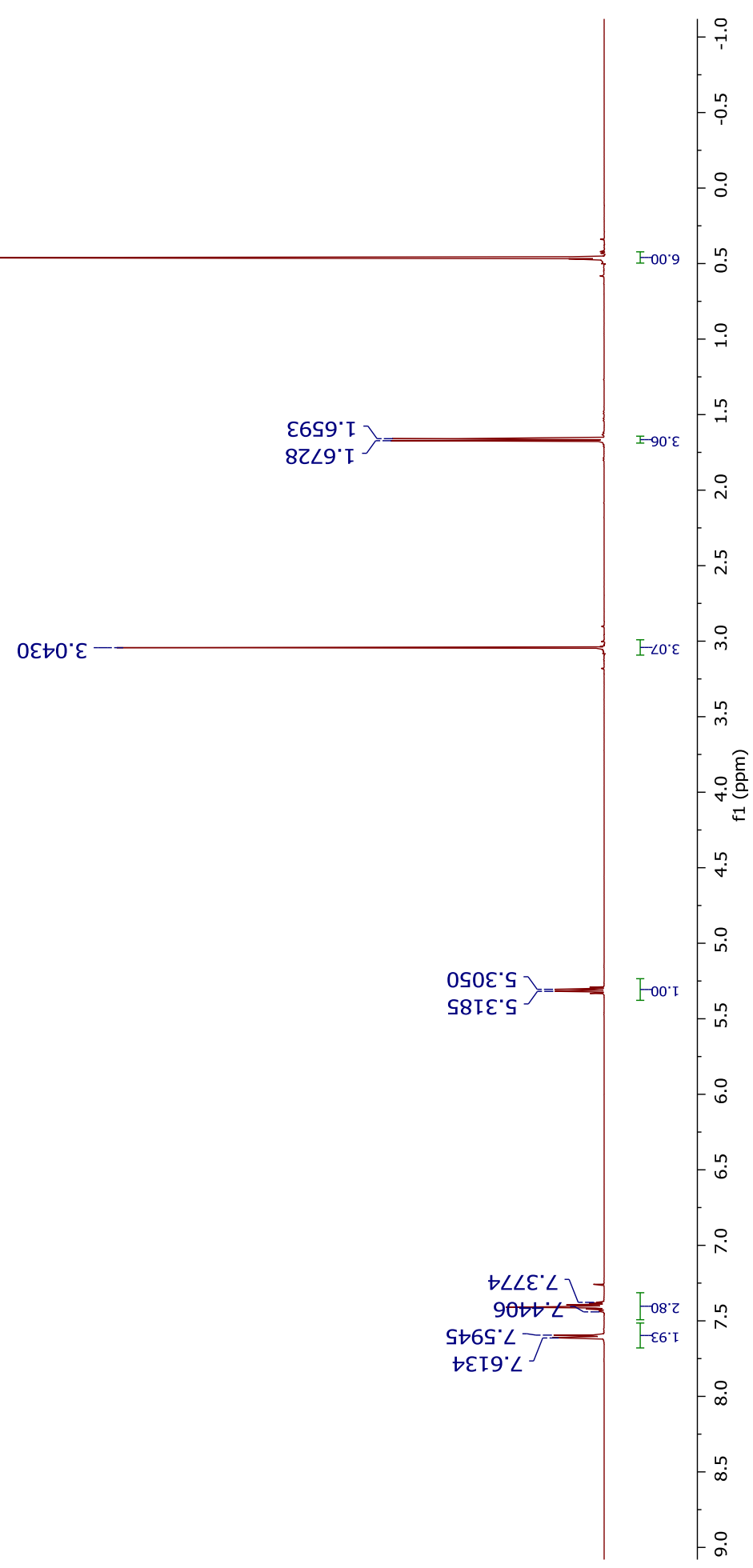




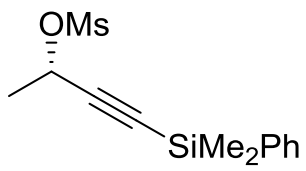

(S)-12

$\mathrm{CDCl}_{3}$ $125 \mathrm{MHz}{ }^{13} \mathrm{C}$

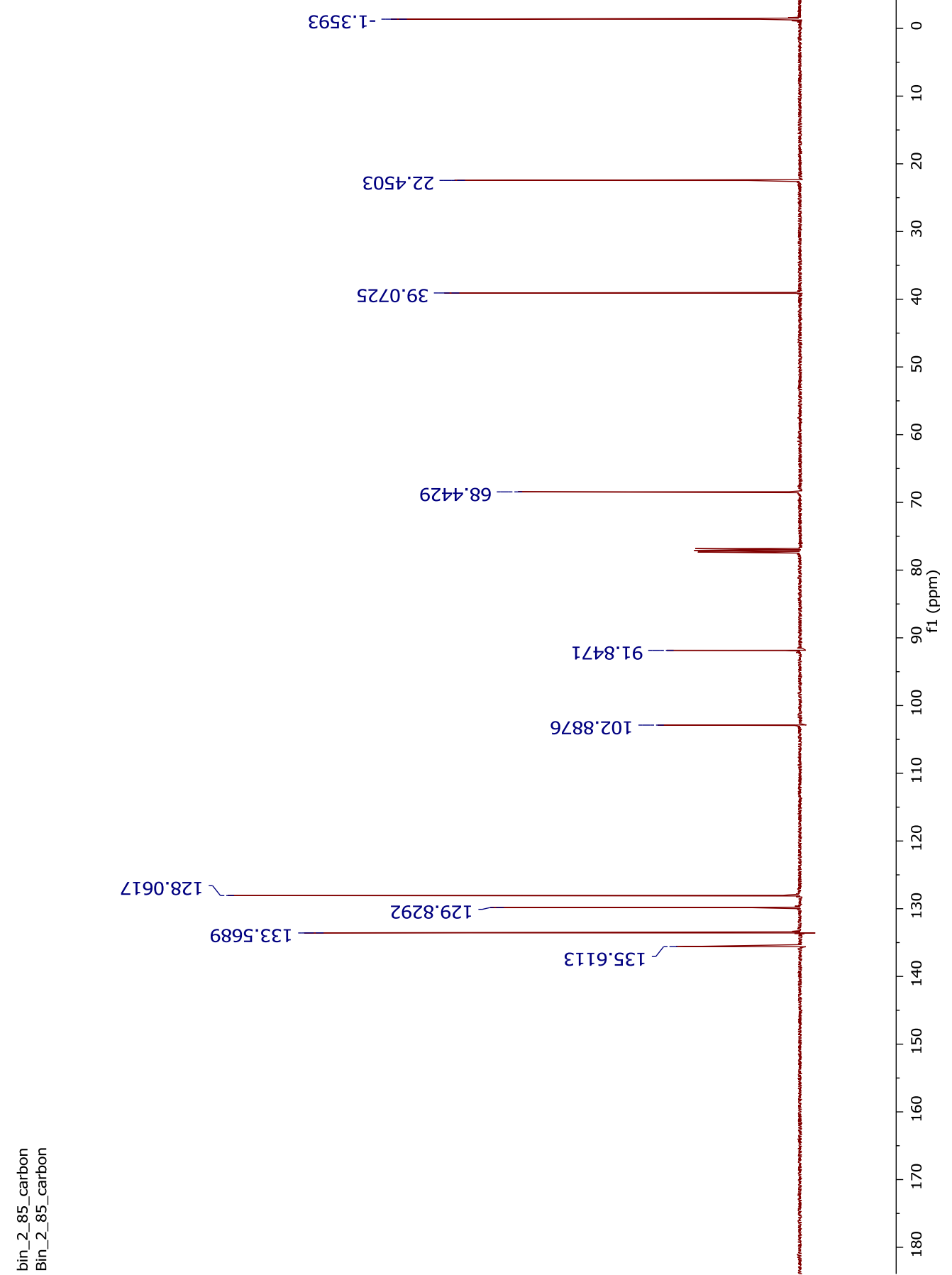




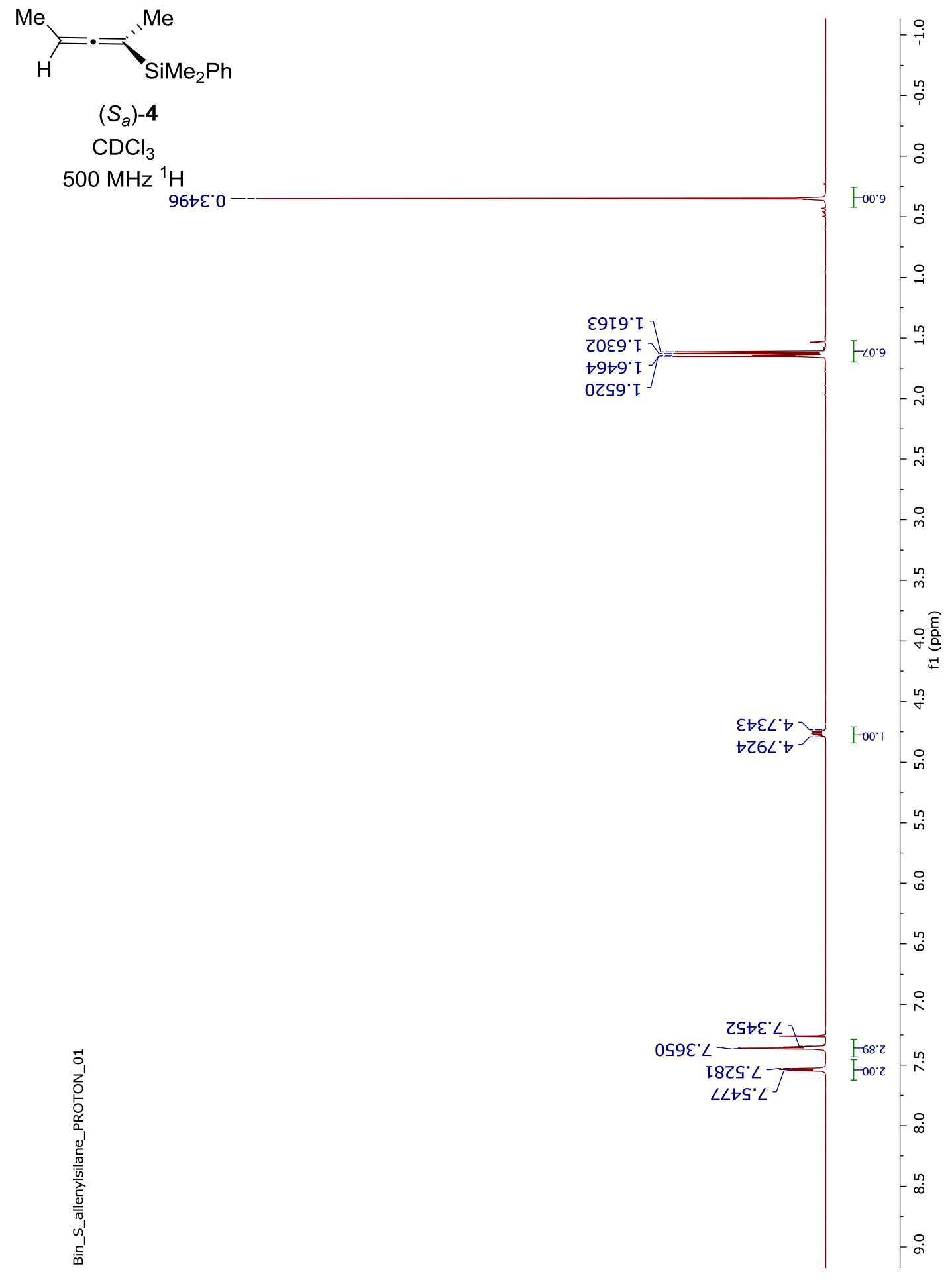



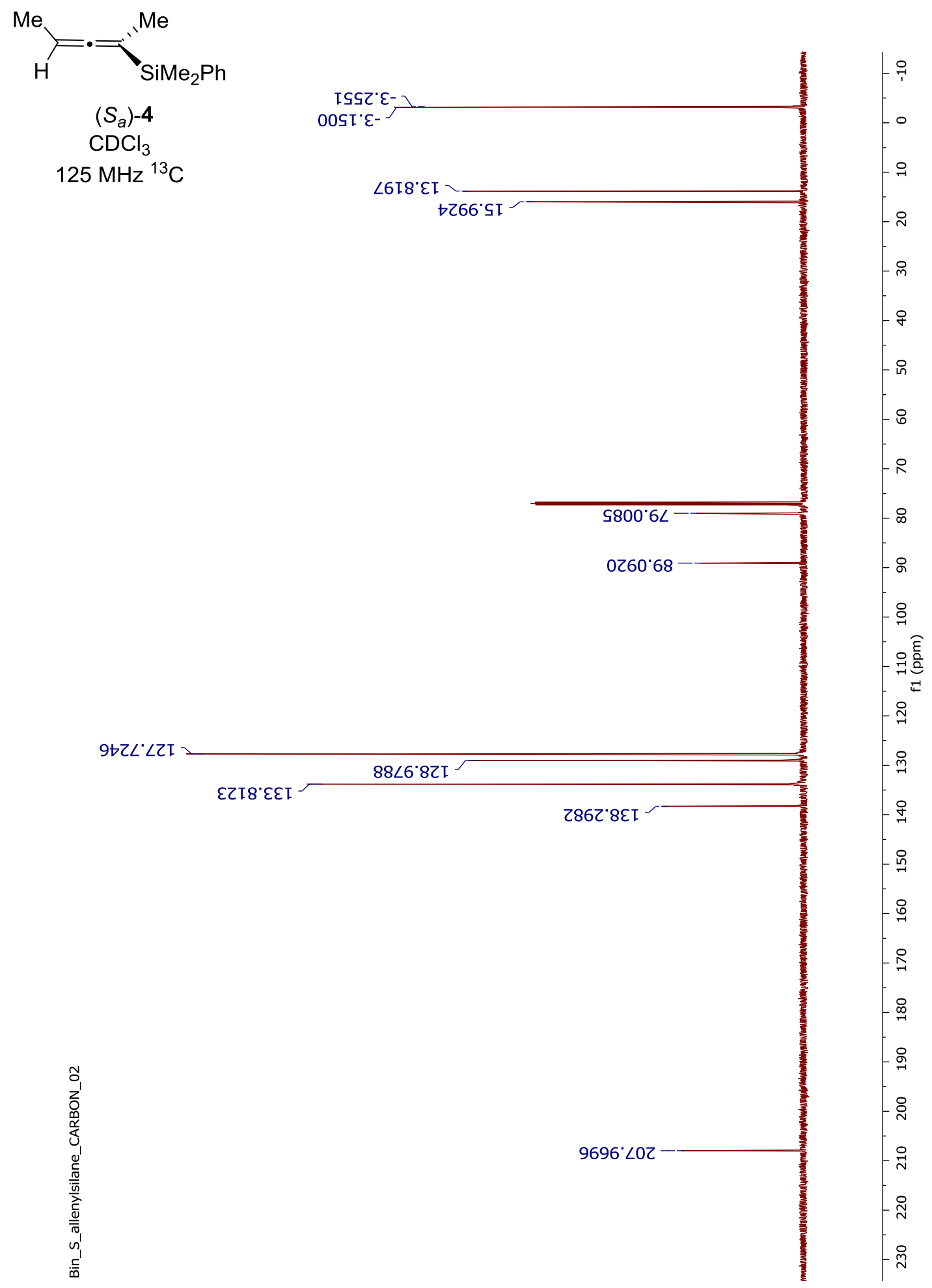


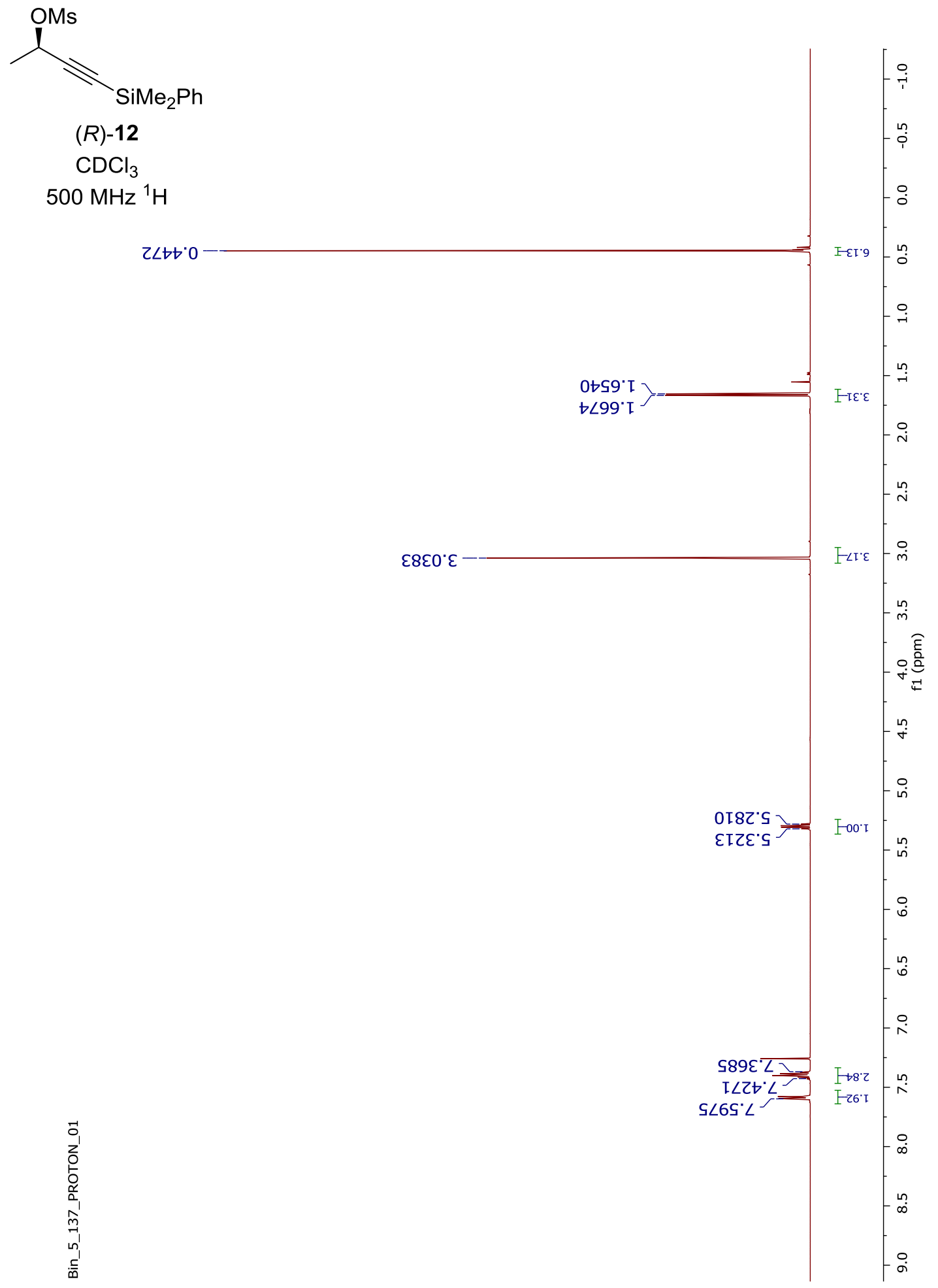



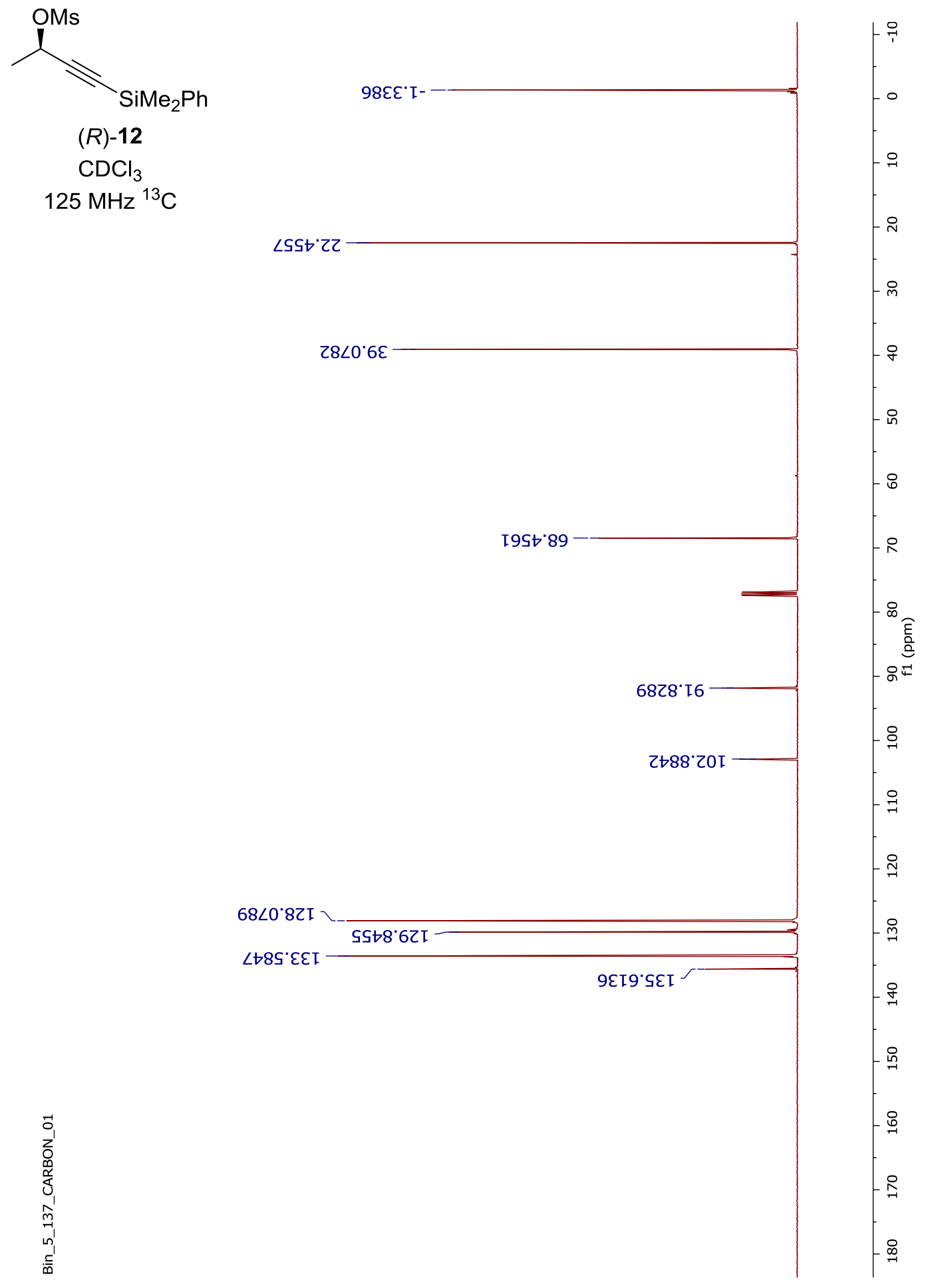


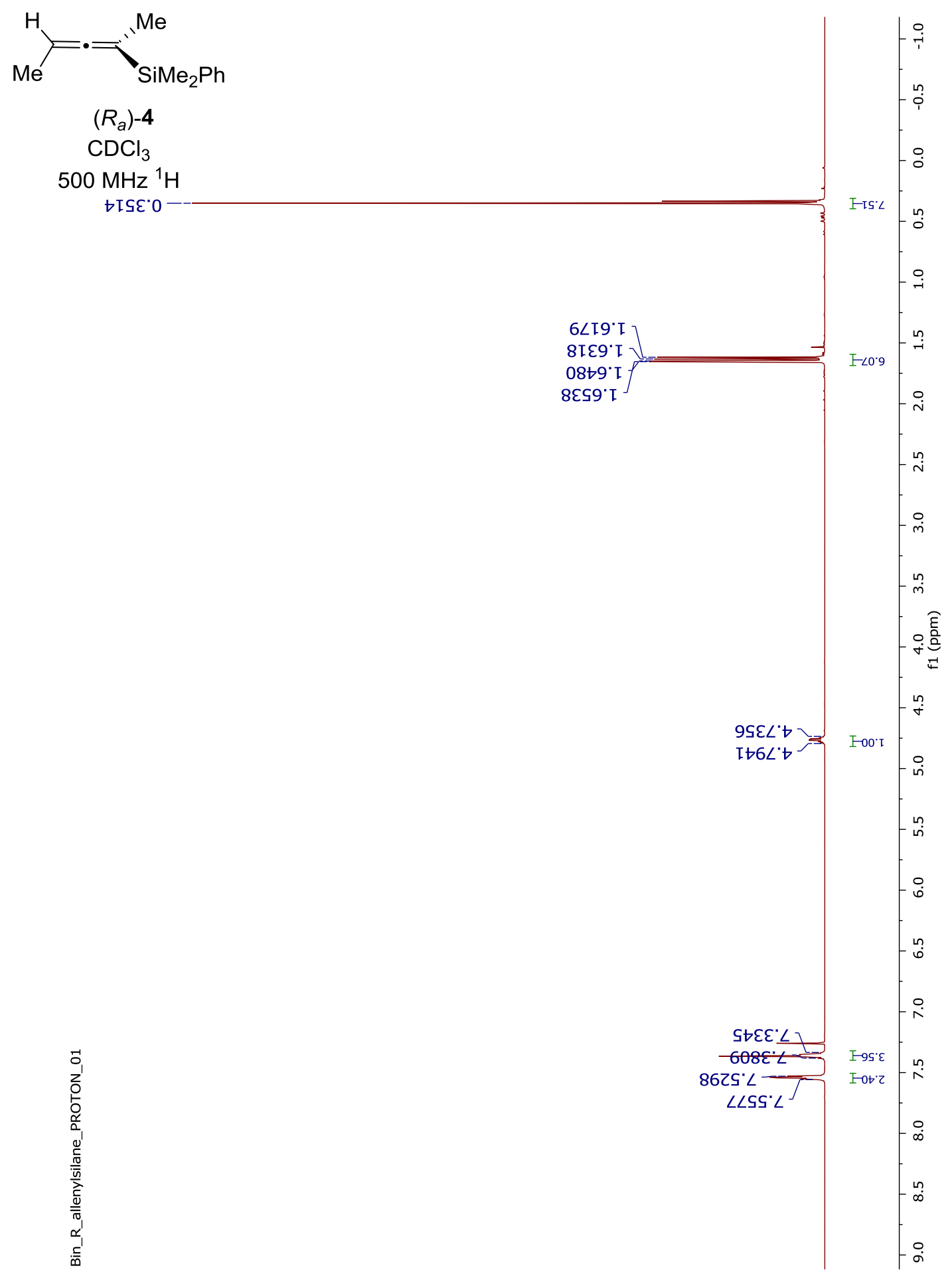


<smiles>C/C=C(\C)C(C)/C=C\c1ccccc1</smiles>

$\left(R_{a}\right)-4$
$\mathrm{CDCl}_{3}$
$500 \mathrm{MHz}^{13} \mathrm{C}$

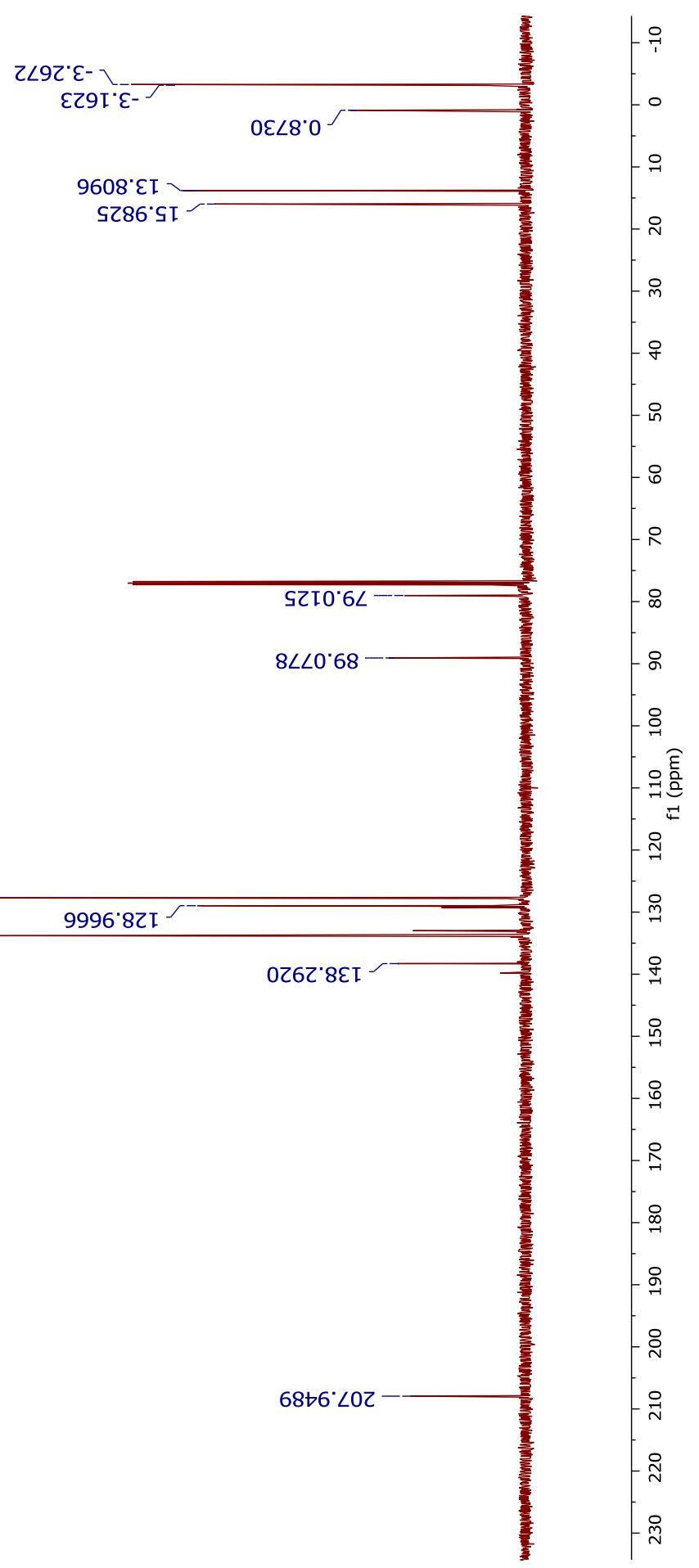

SI-35 

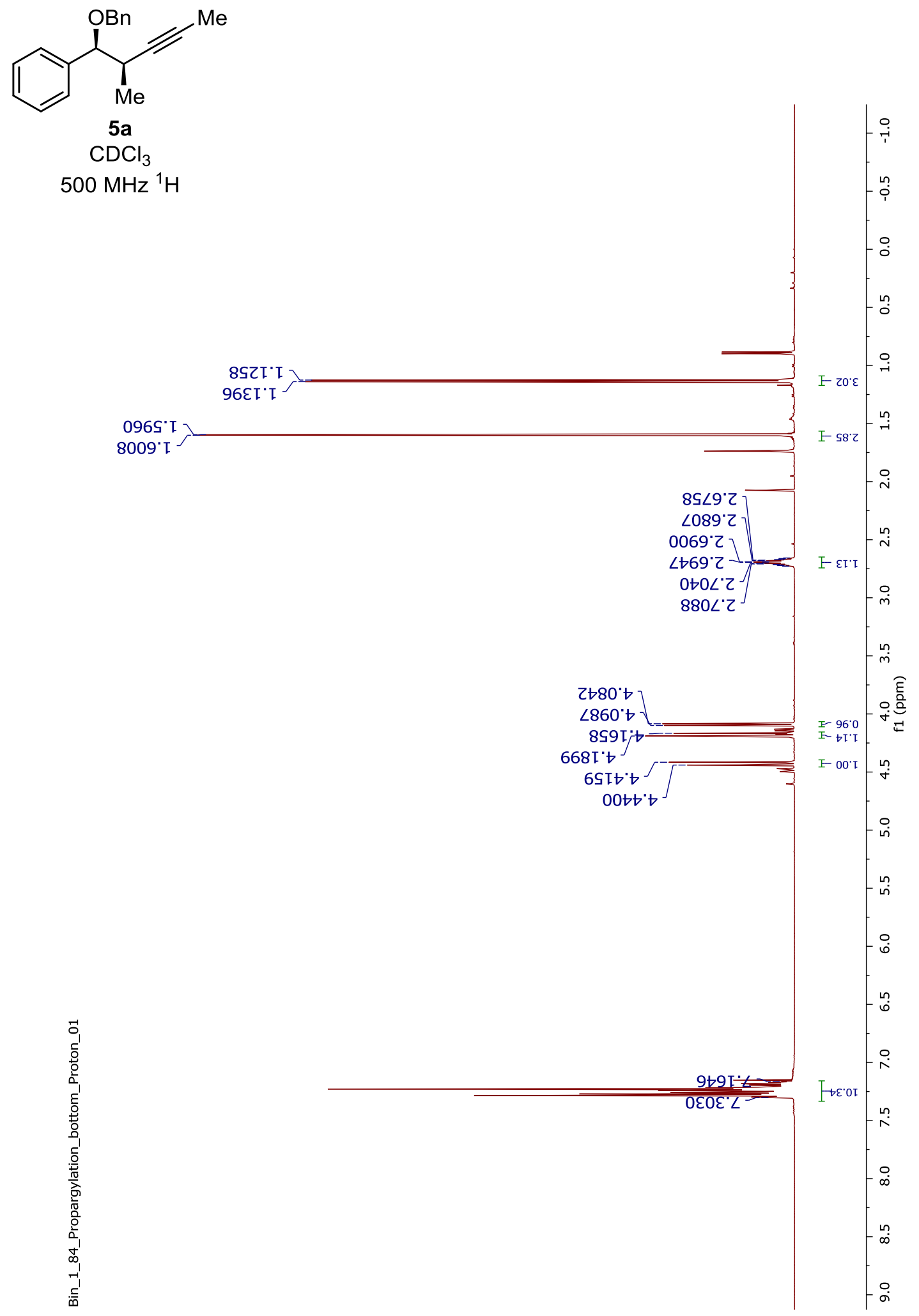


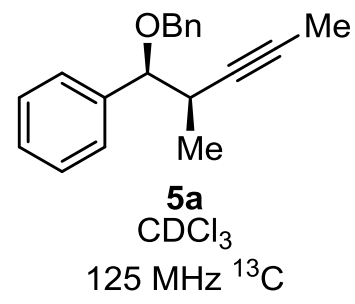

I0๑乙" $৮ Z I$ IS8Z'

โ086. $\varepsilon Z I$ $\angle 9 Z t^{\circ} \mathrm{CL}$ $9 \angle I{ }^{\circ} \triangleright Z I$ $\angle L L L{ }^{\circ}{ }^{\prime} I^{\int}$

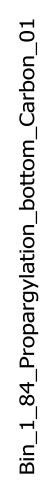

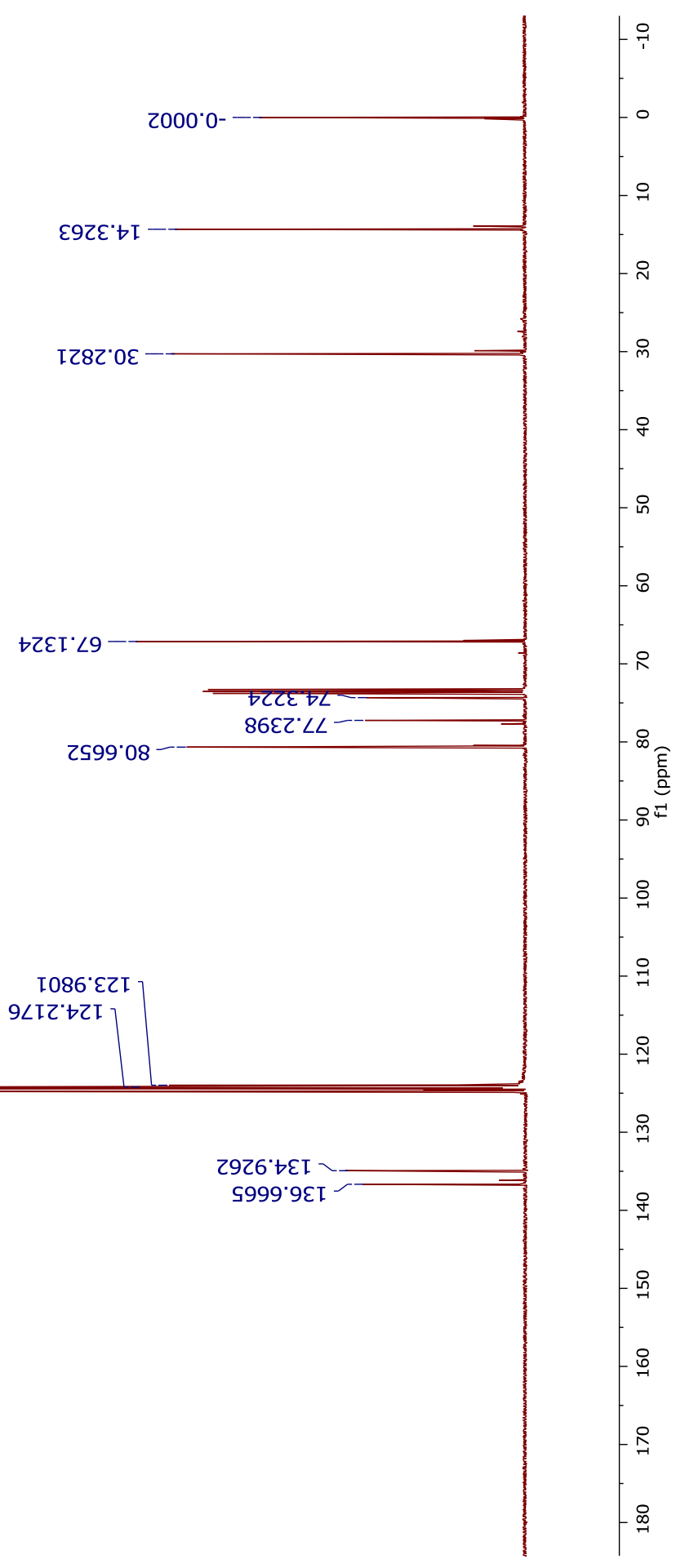




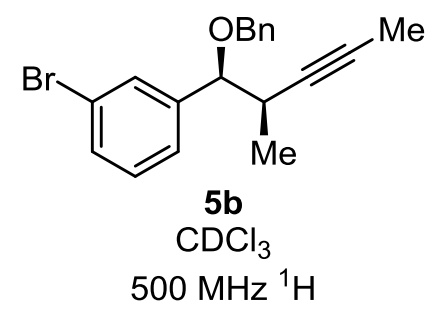

$500 \mathrm{MHz}{ }^{1} \mathrm{H}$

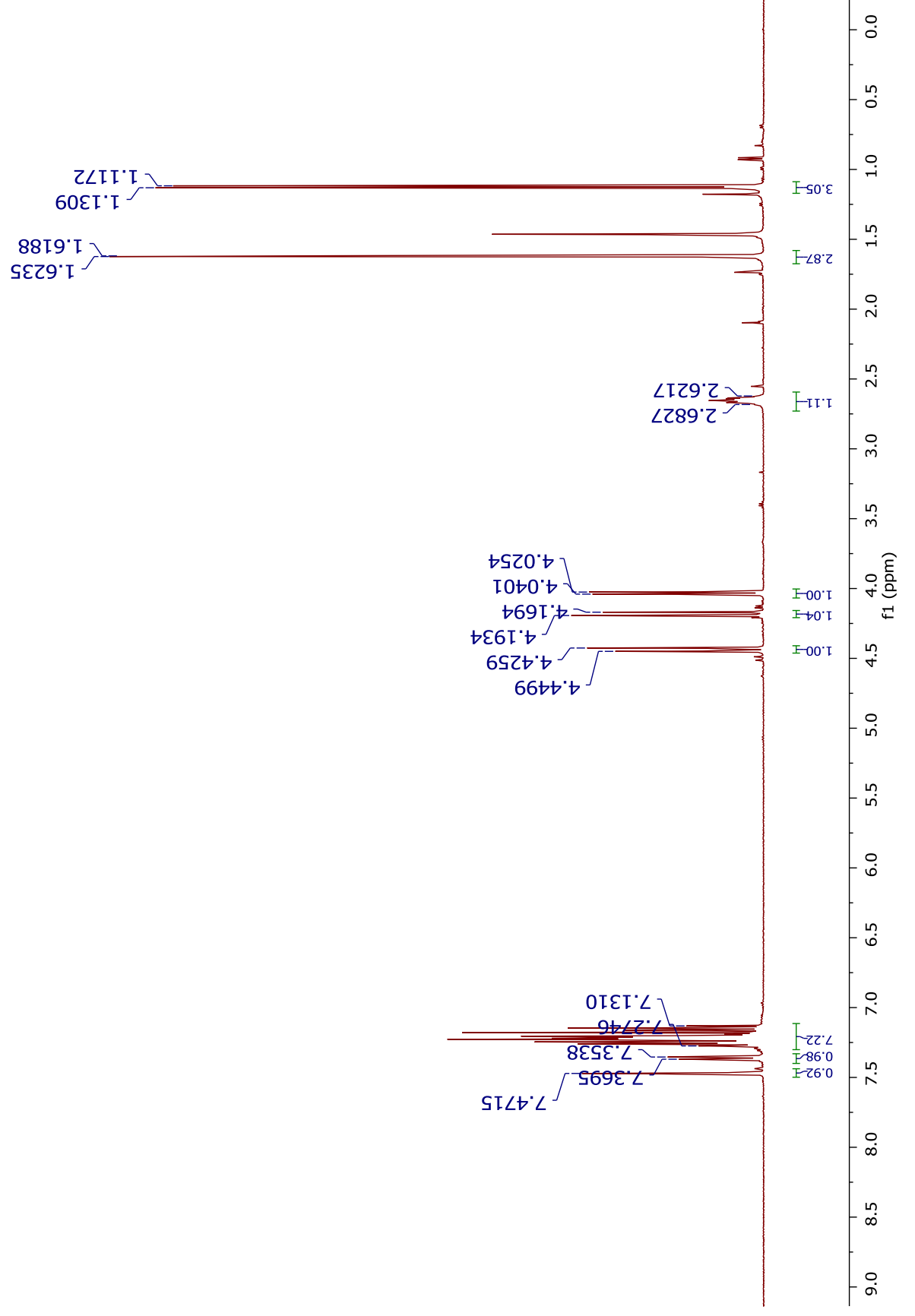




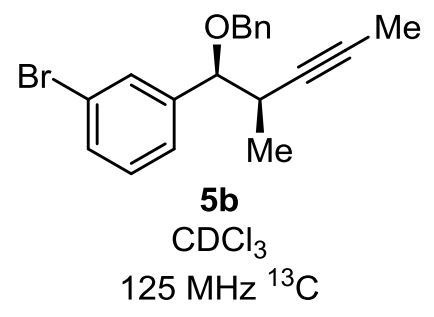

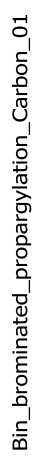

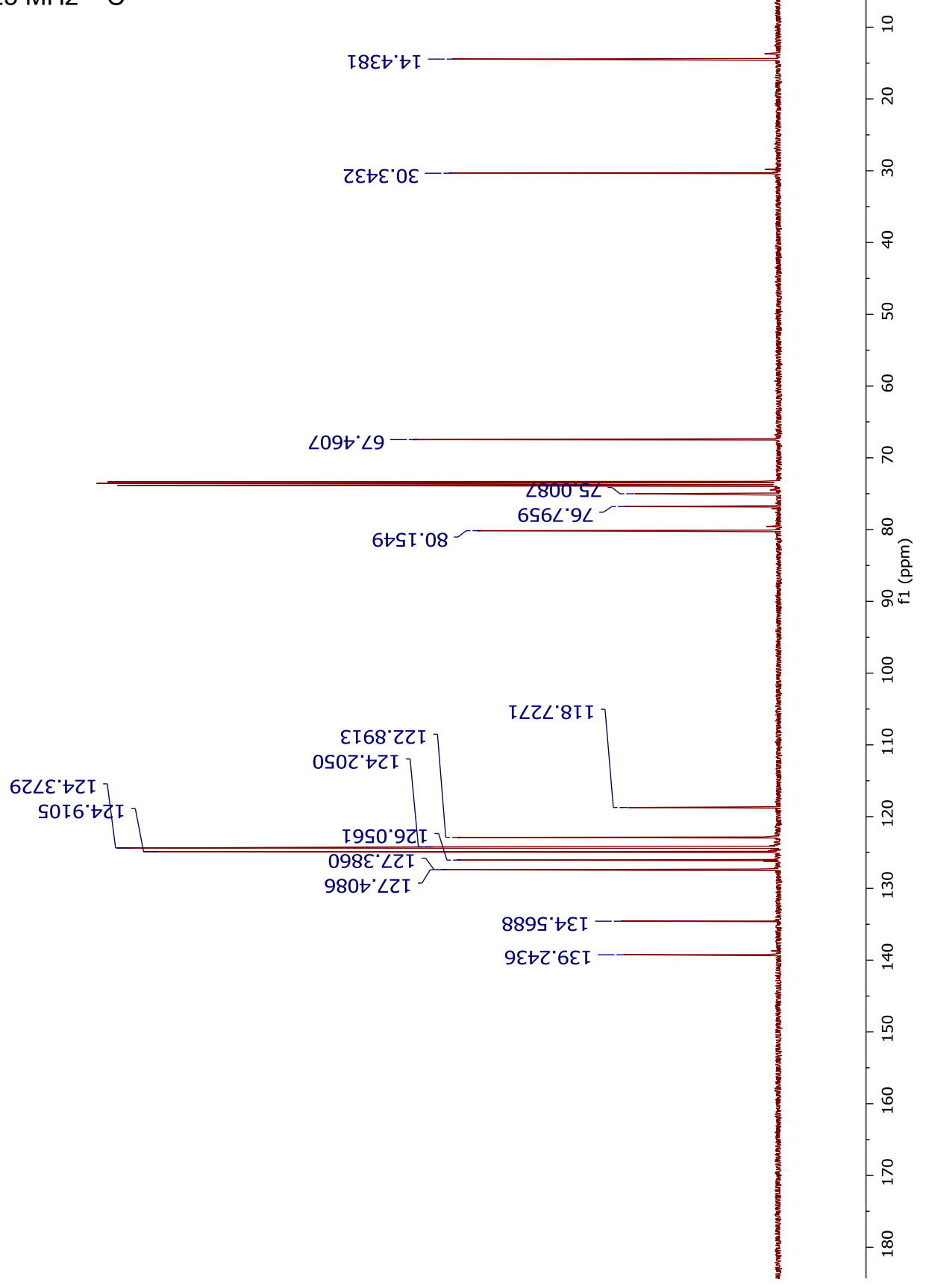




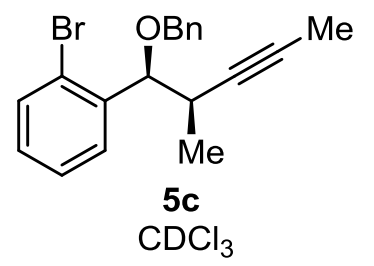

$500 \mathrm{MHz}{ }^{1} \mathrm{H}$

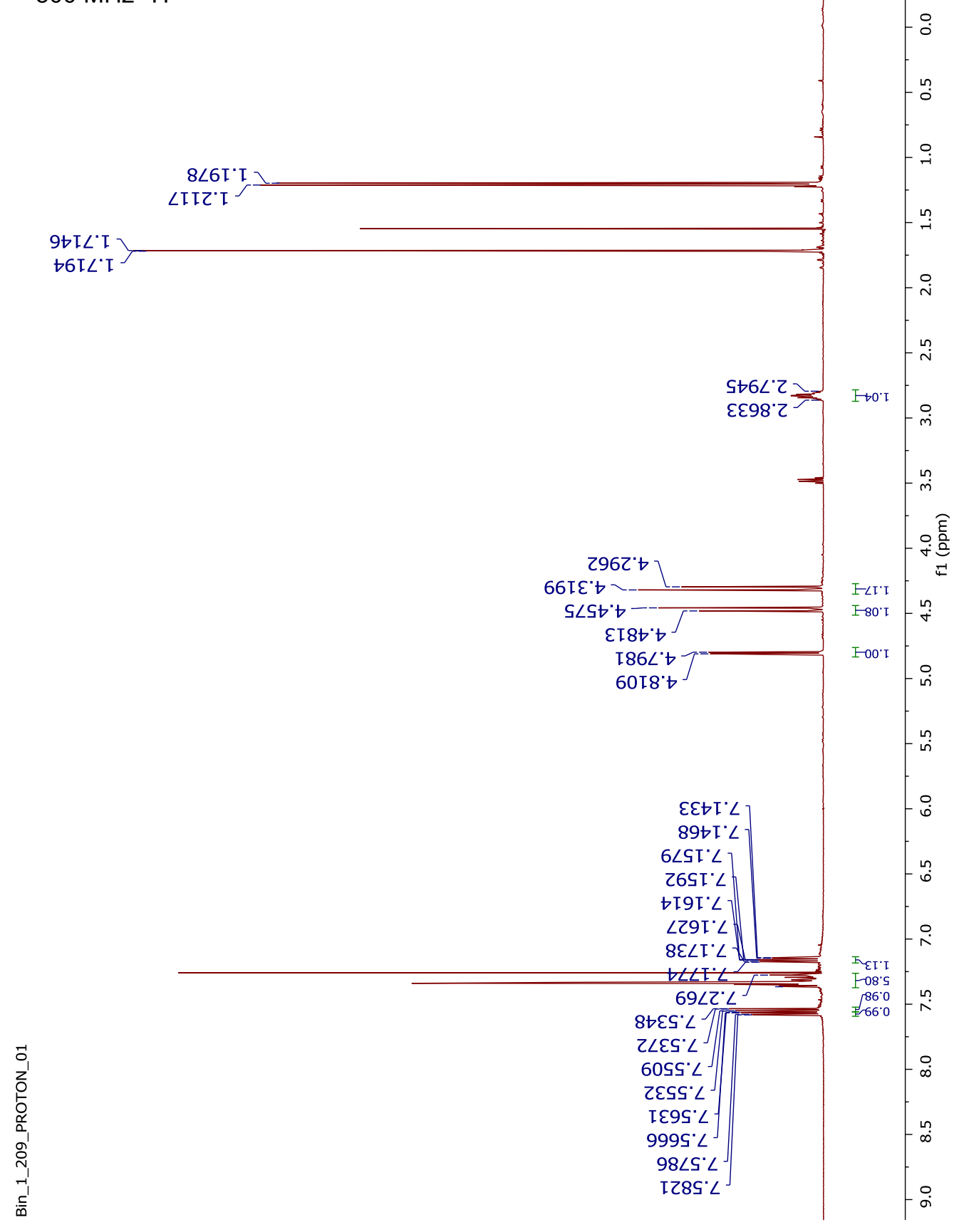



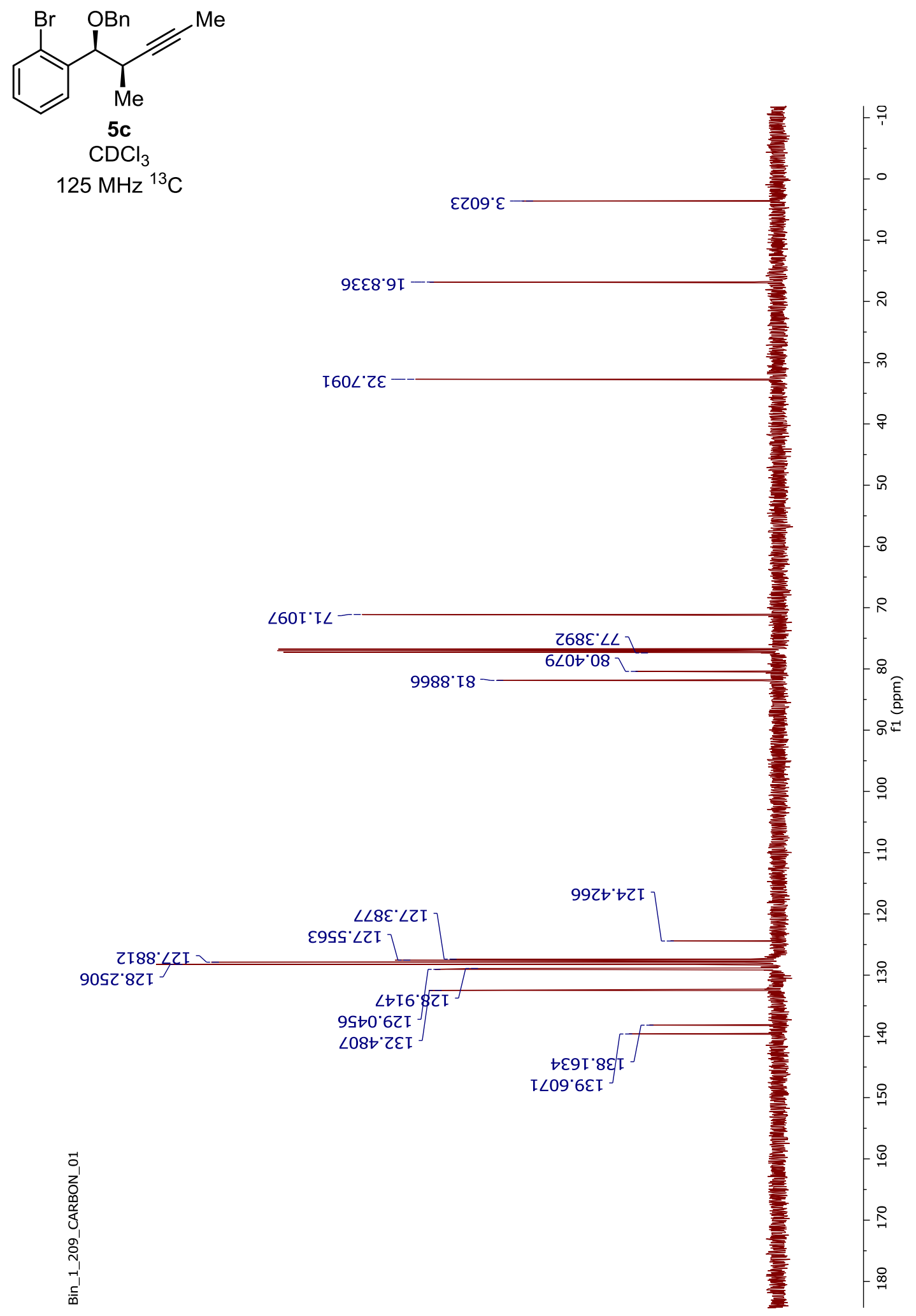

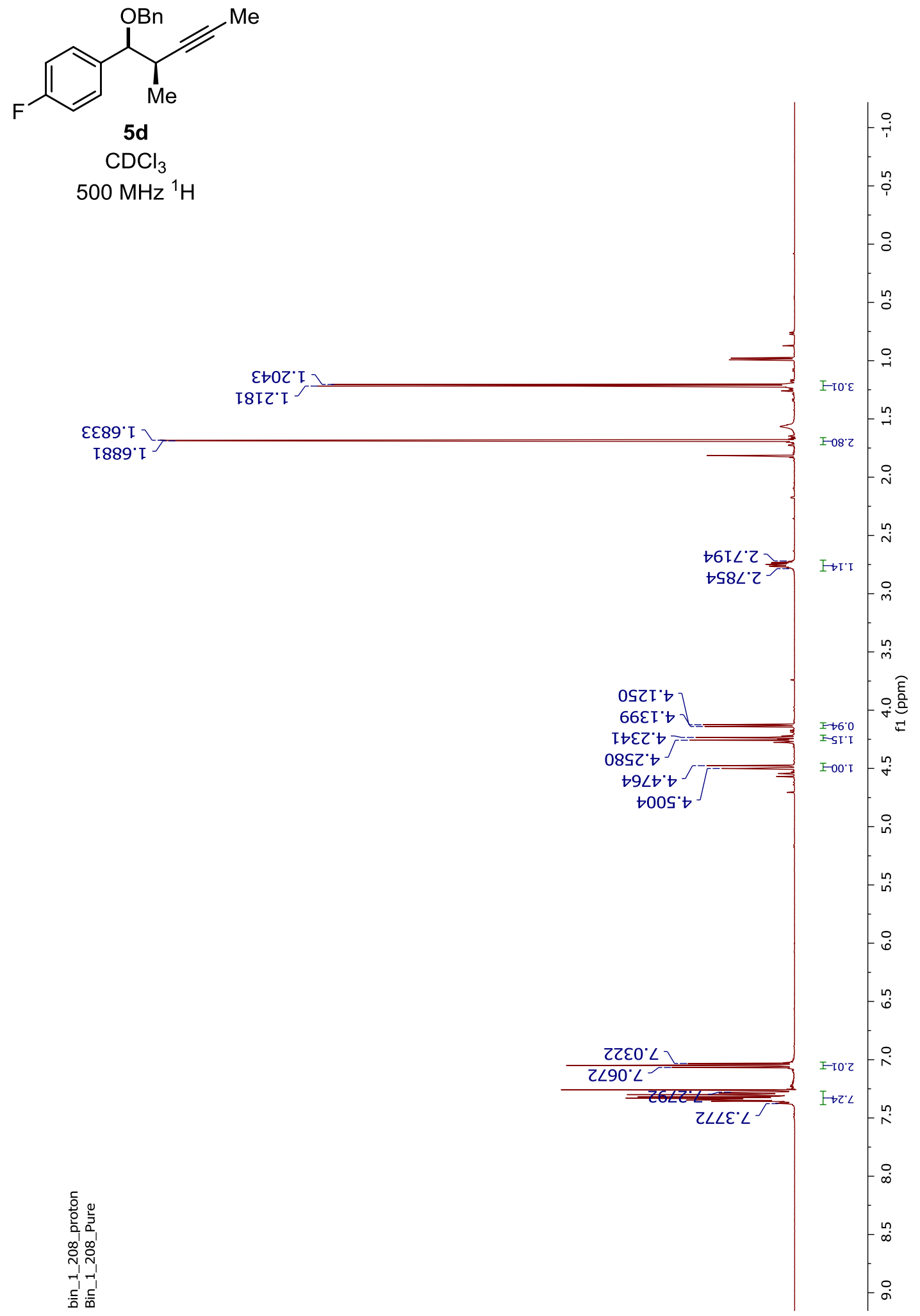

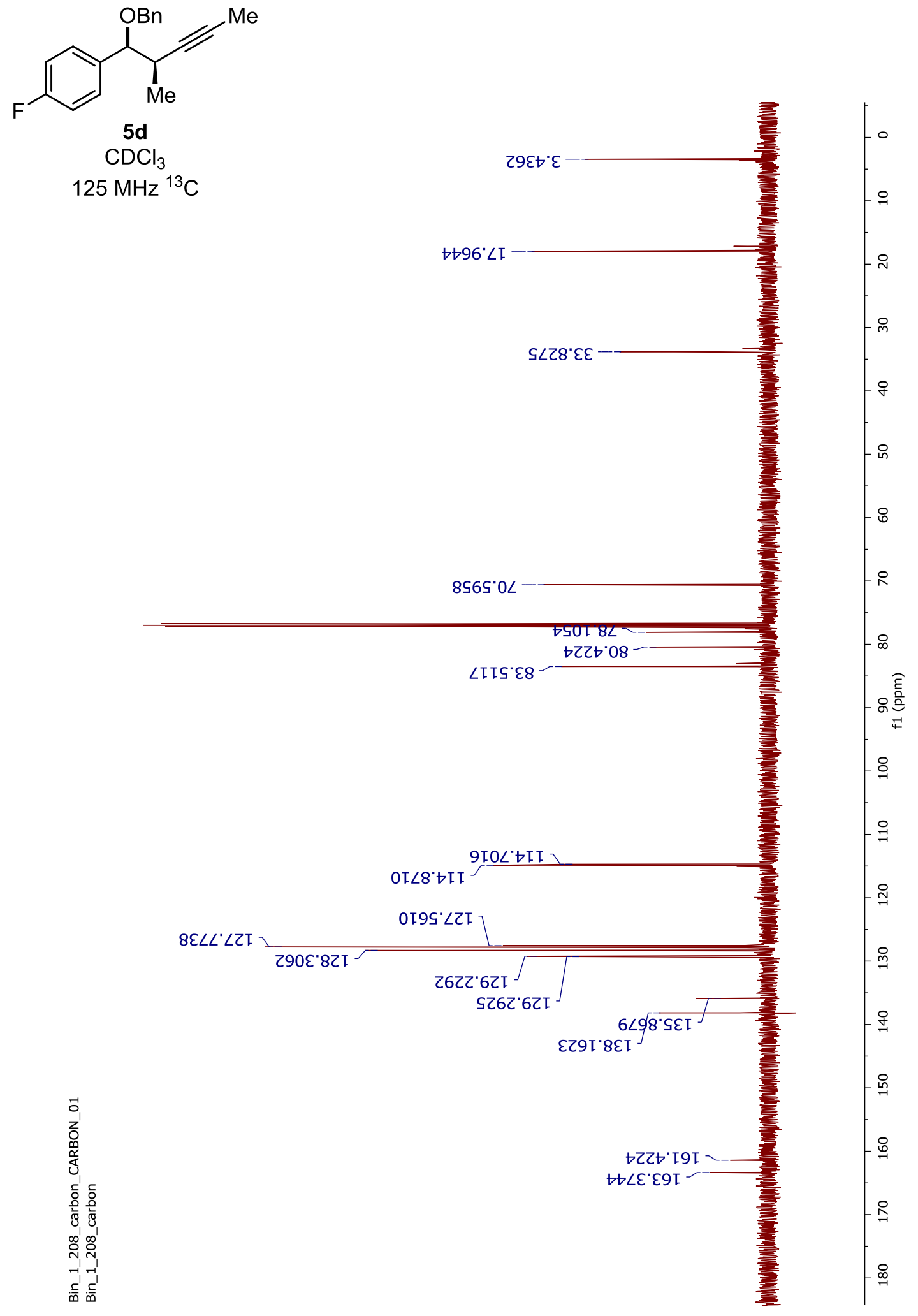


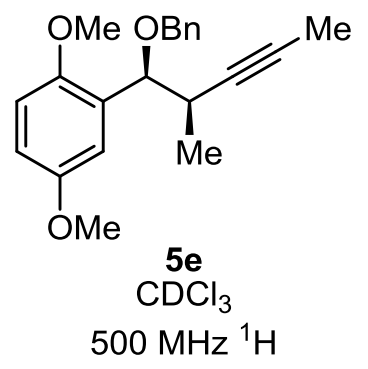

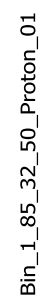

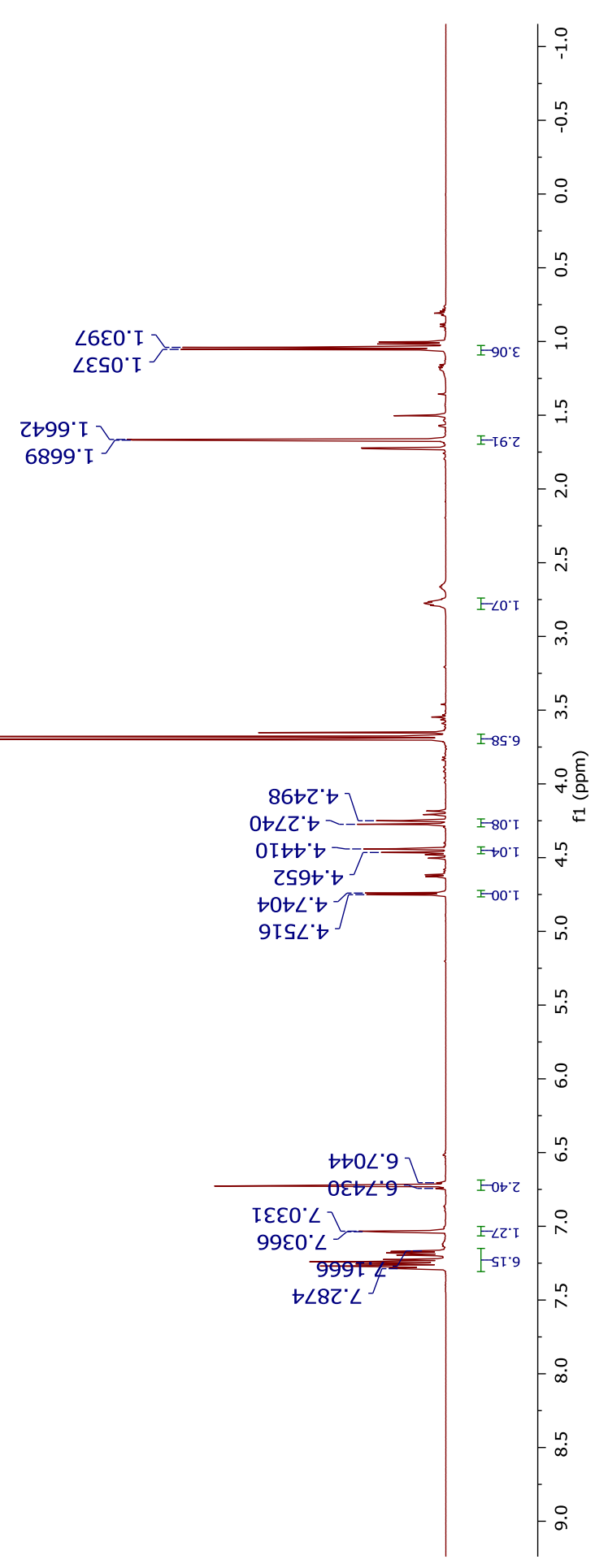




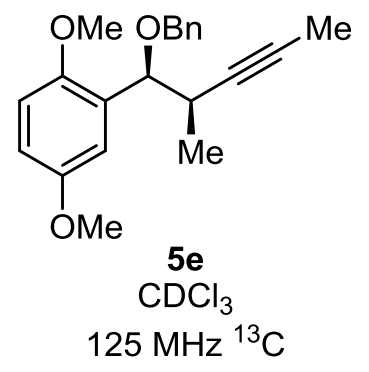

$\angle 9 I I^{\circ} \downarrow C I$ 8IटS'†ZI

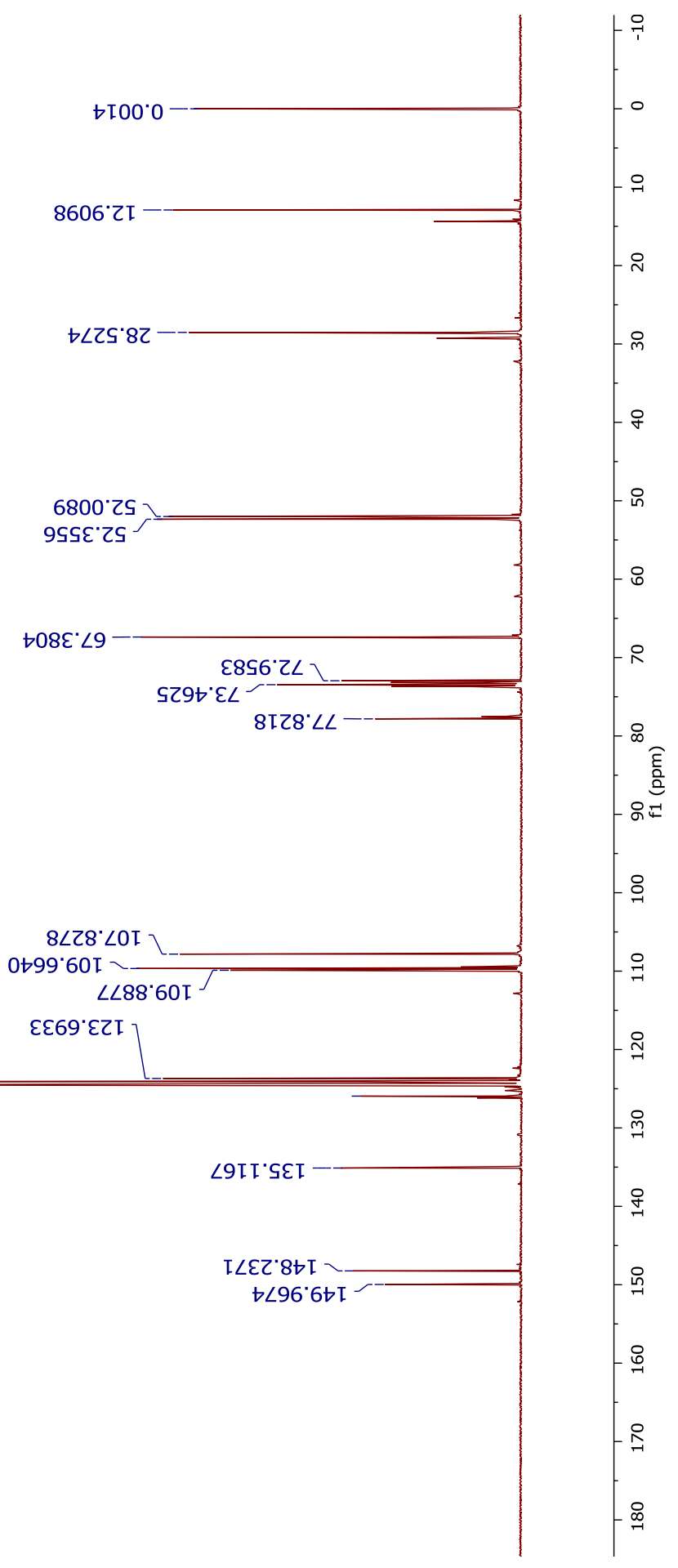




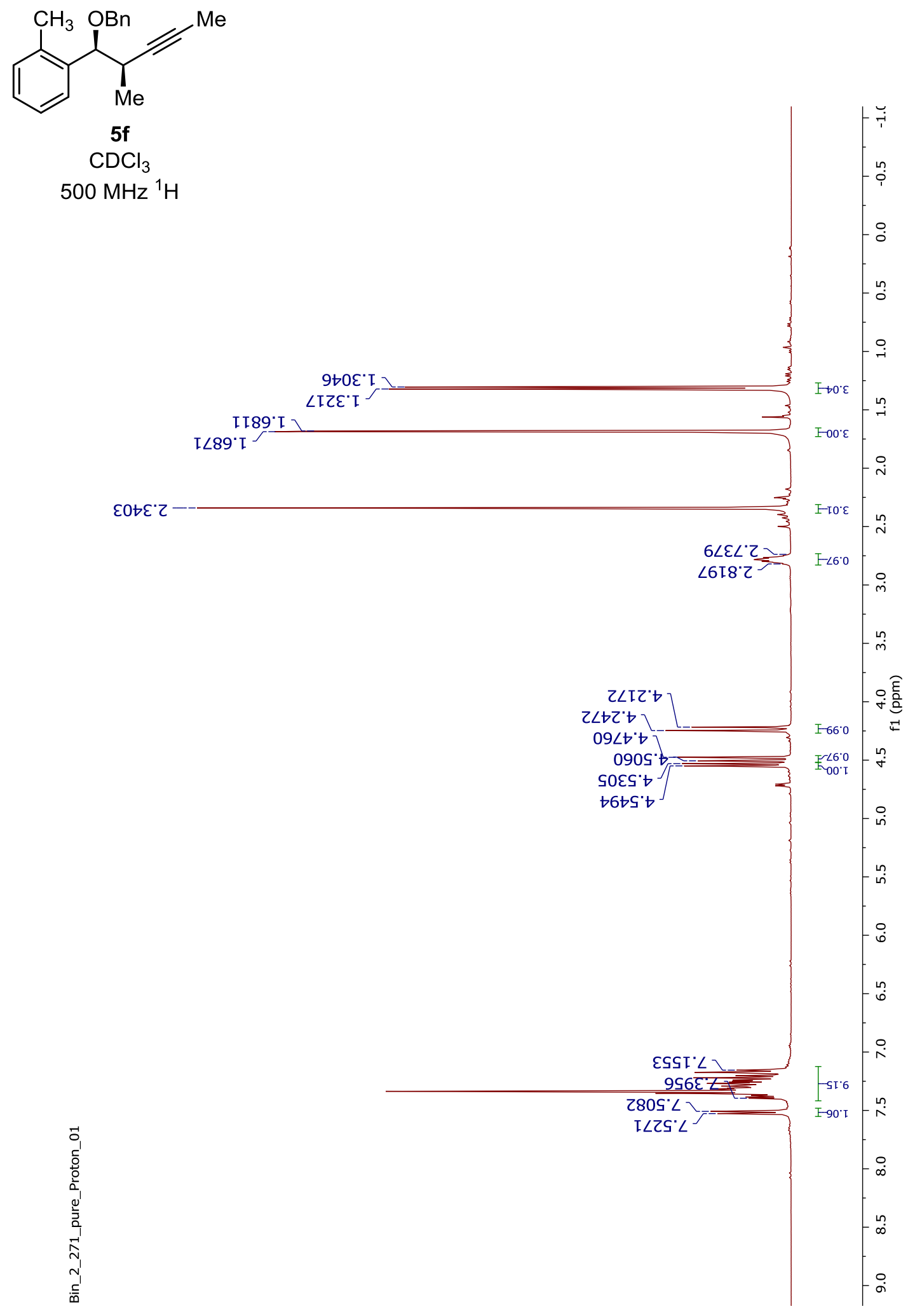



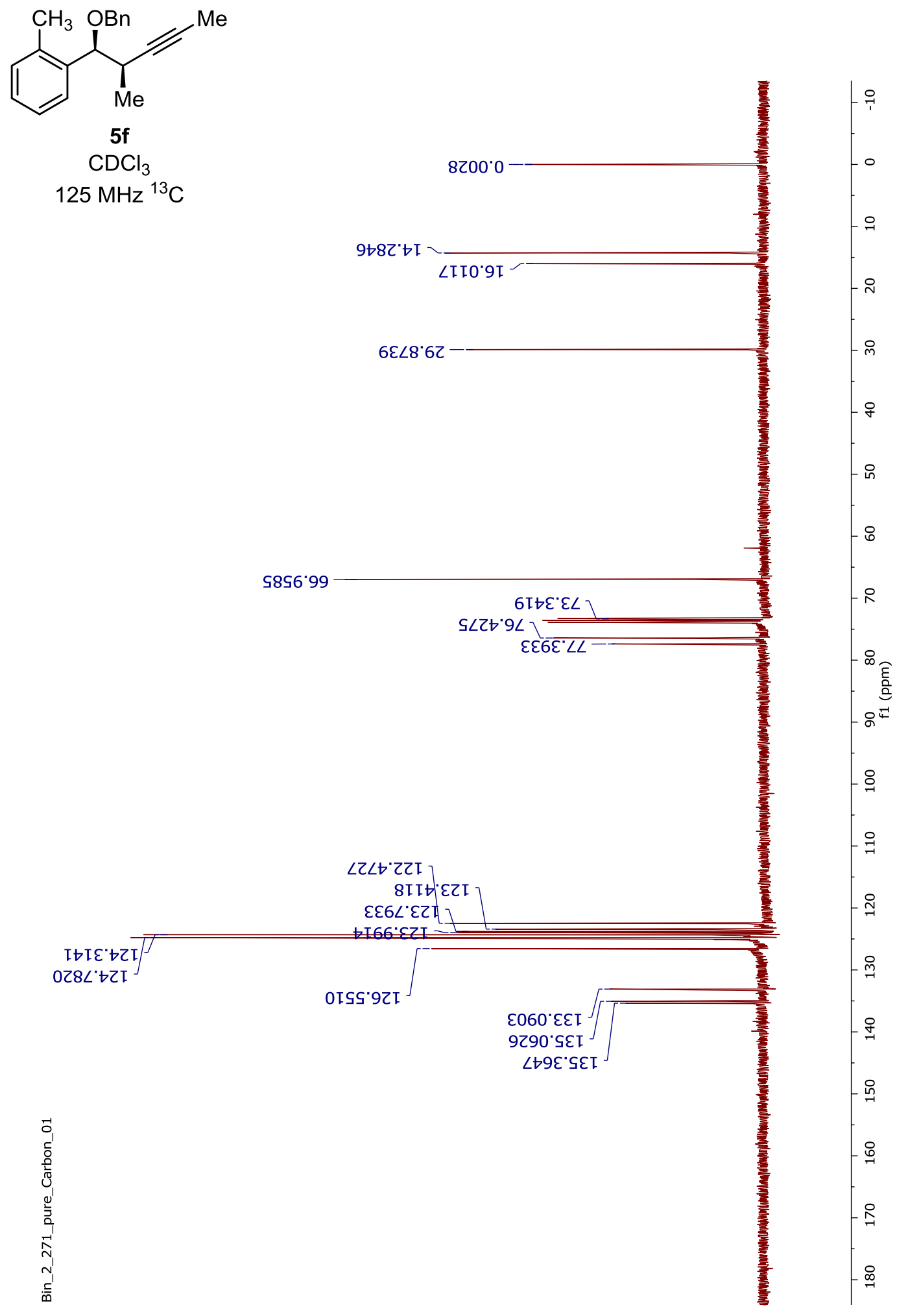


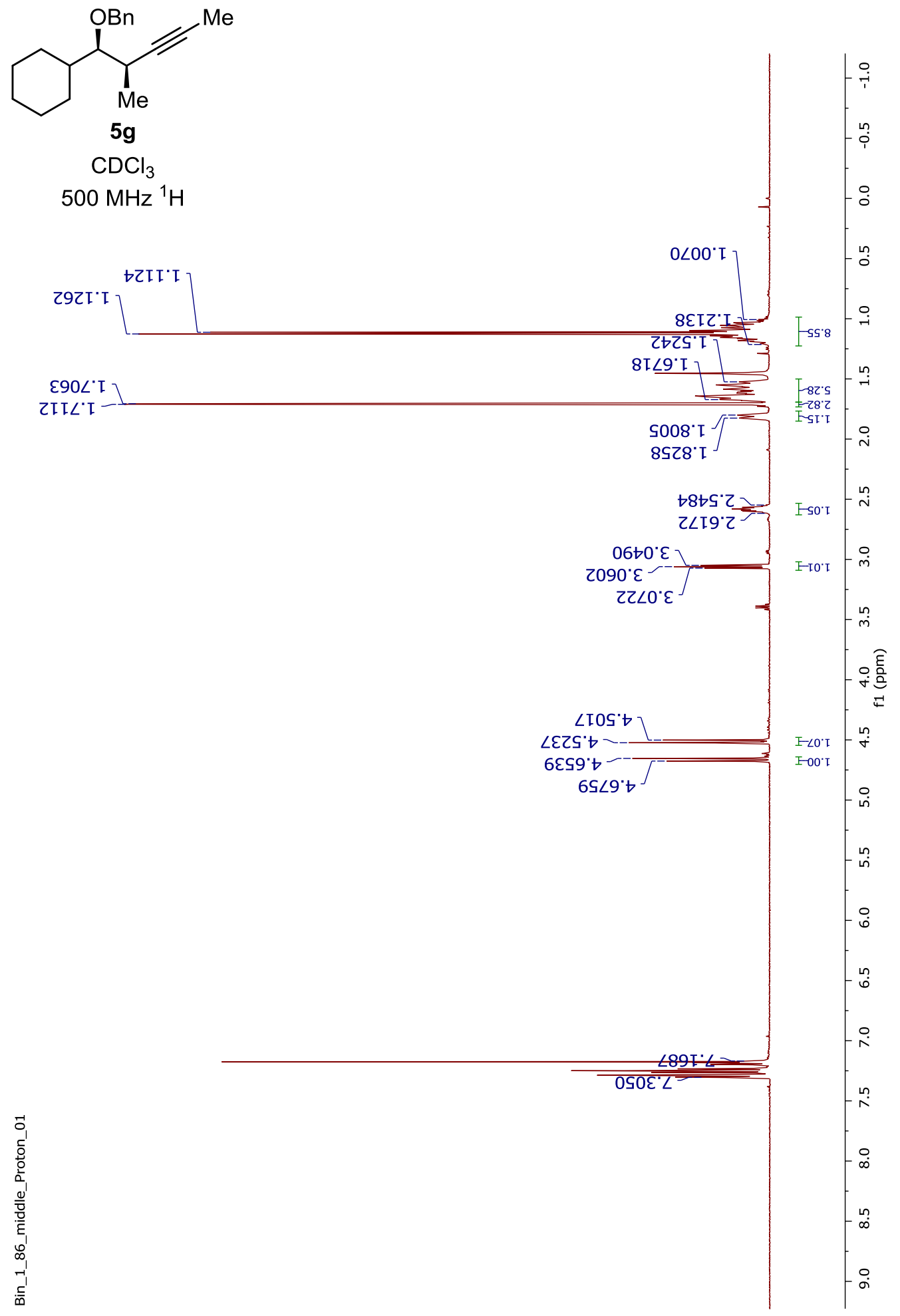



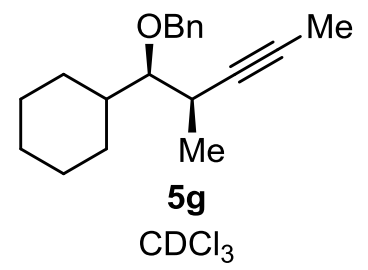

$125 \mathrm{MHz}{ }^{13} \mathrm{C}$

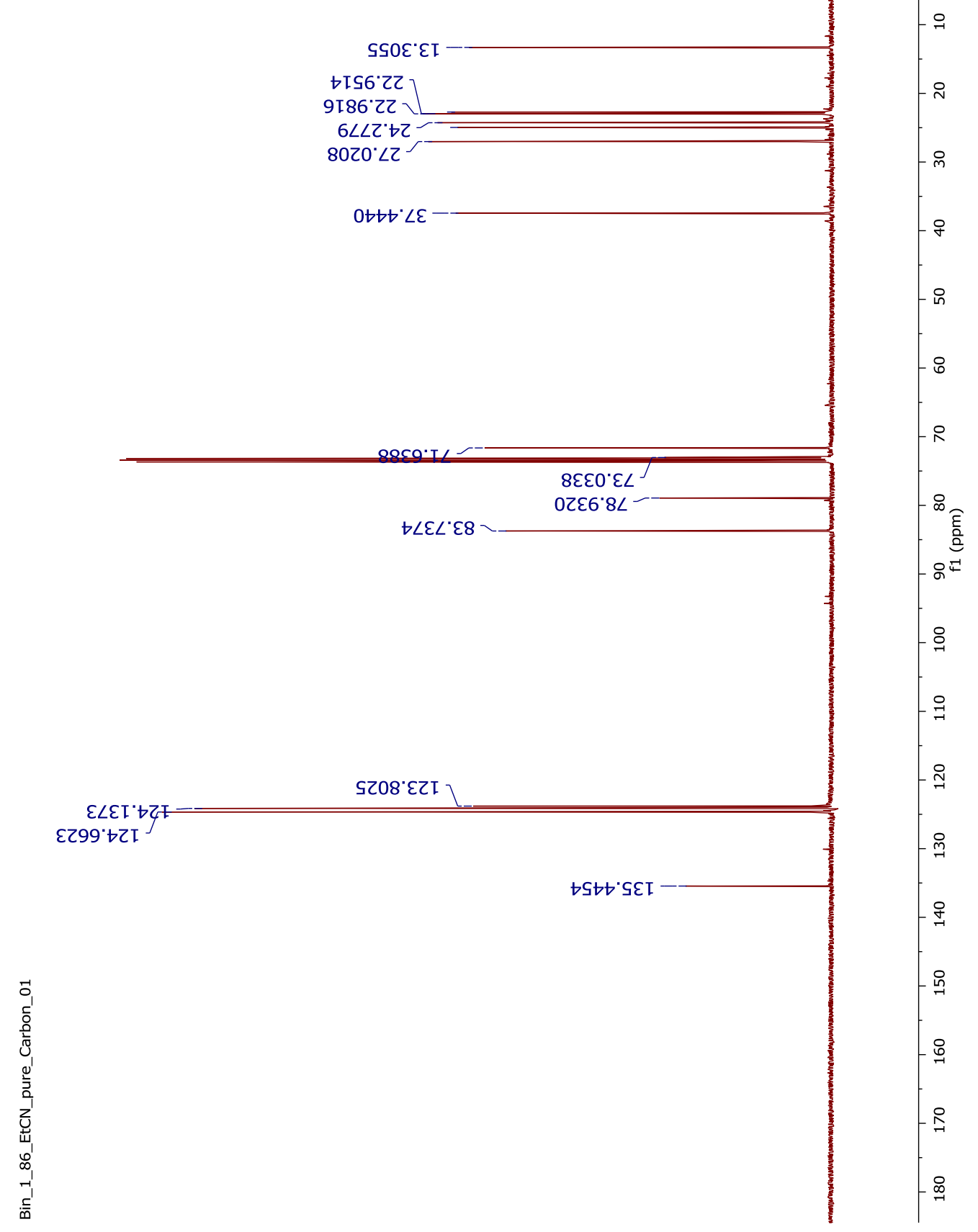



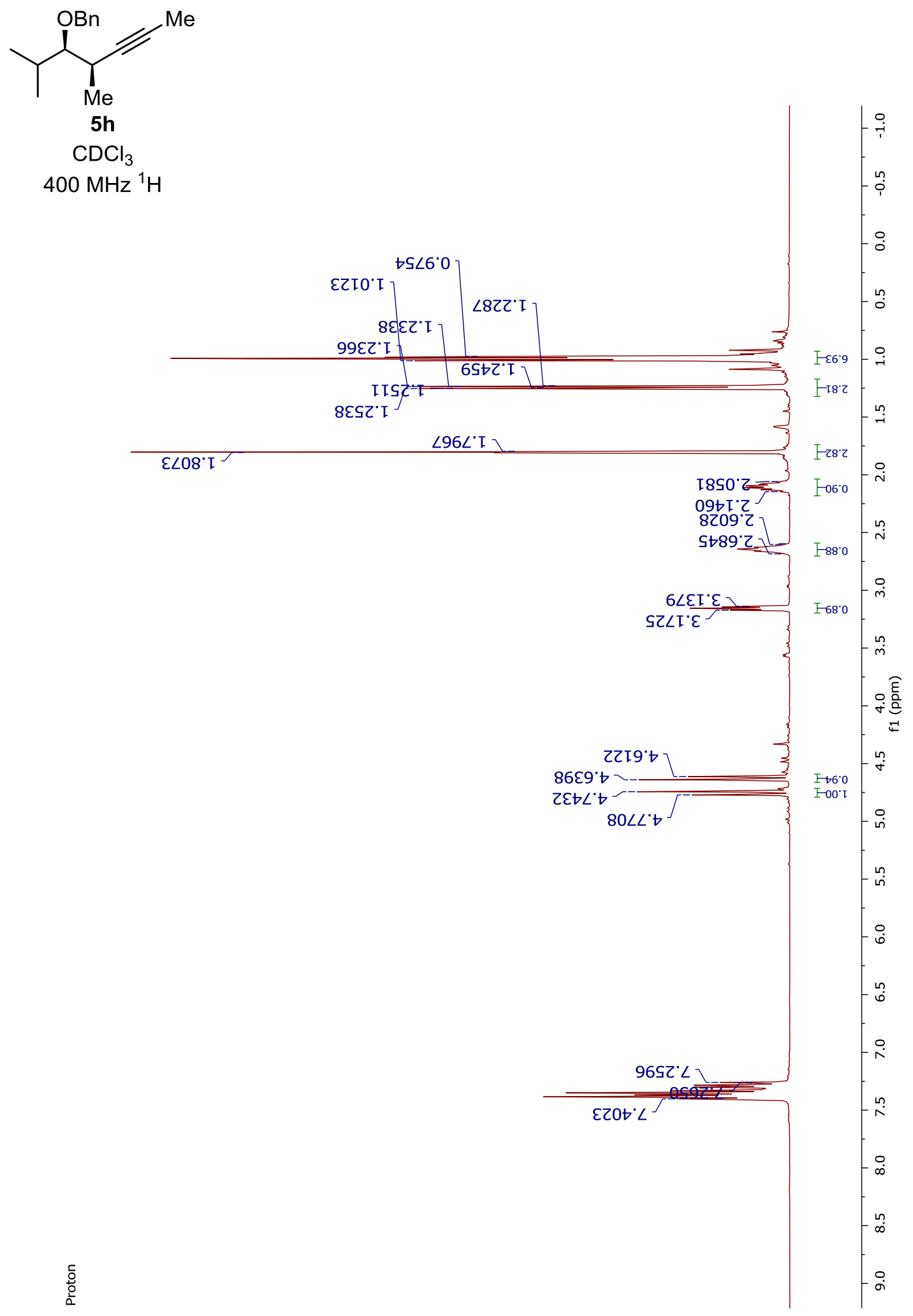

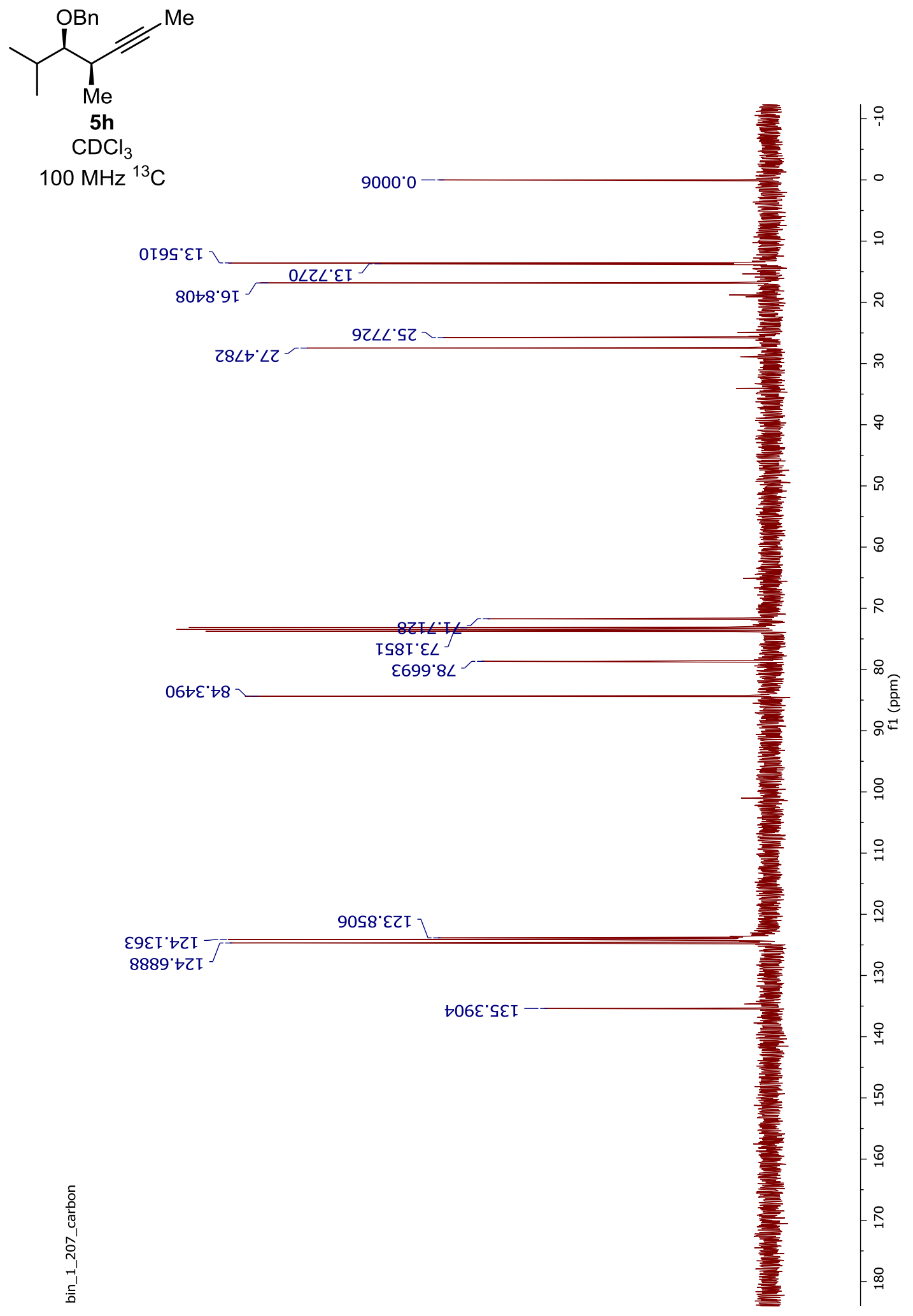
<smiles>CC#CC(C)C(CC(C)C)OCc1ccccc1</smiles>

$5 i$ $\mathrm{CDCl}_{3}$ $500 \mathrm{MHz}{ }^{1} \mathrm{H}$

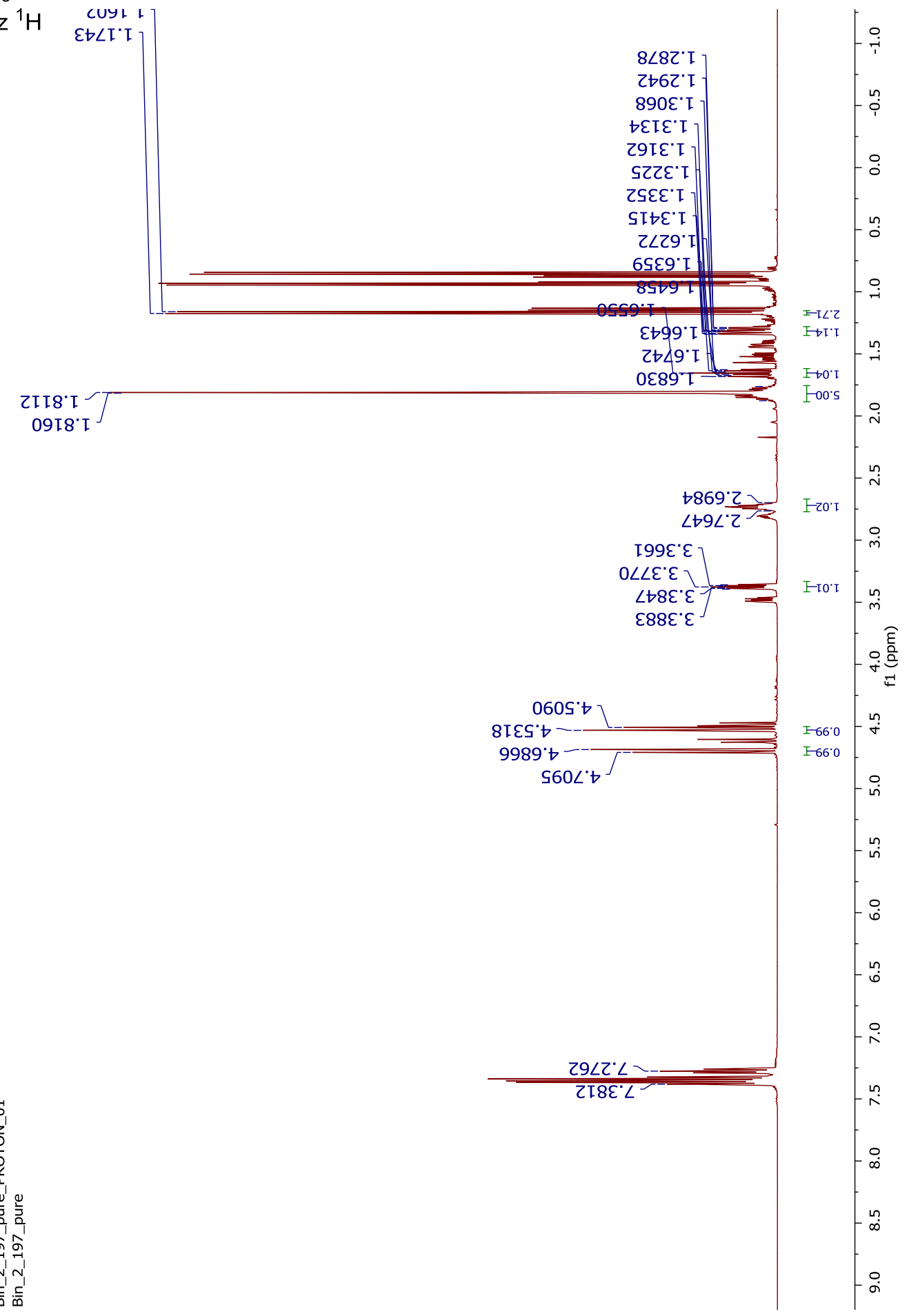




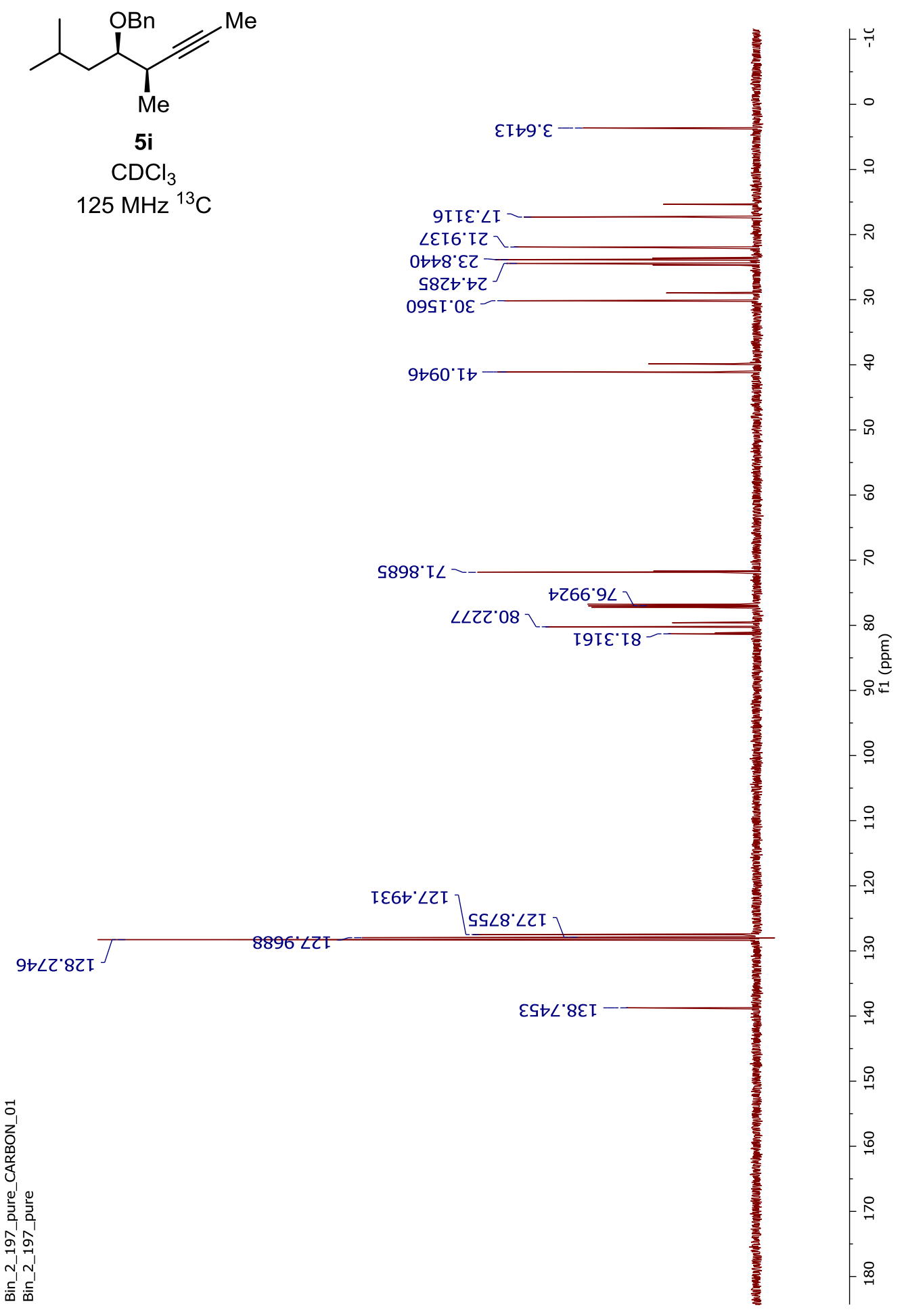




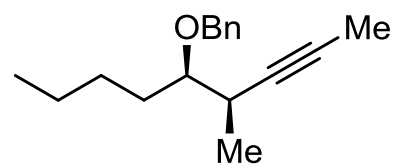

5j

$\mathrm{CDCl}_{3}$

$400 \mathrm{MHz}{ }^{1} \mathrm{H}$

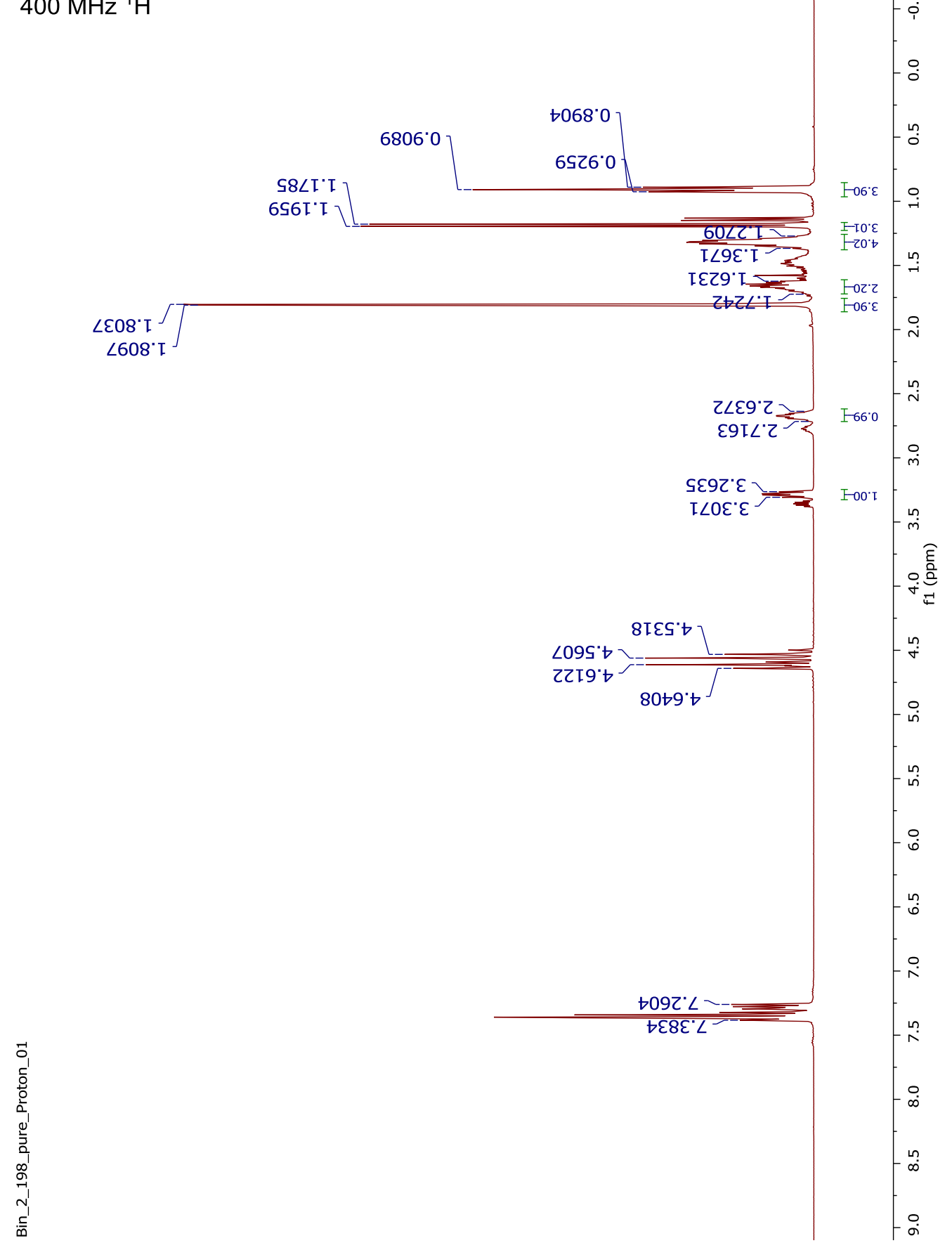




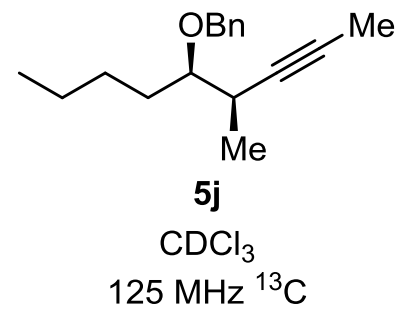

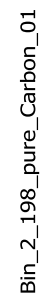

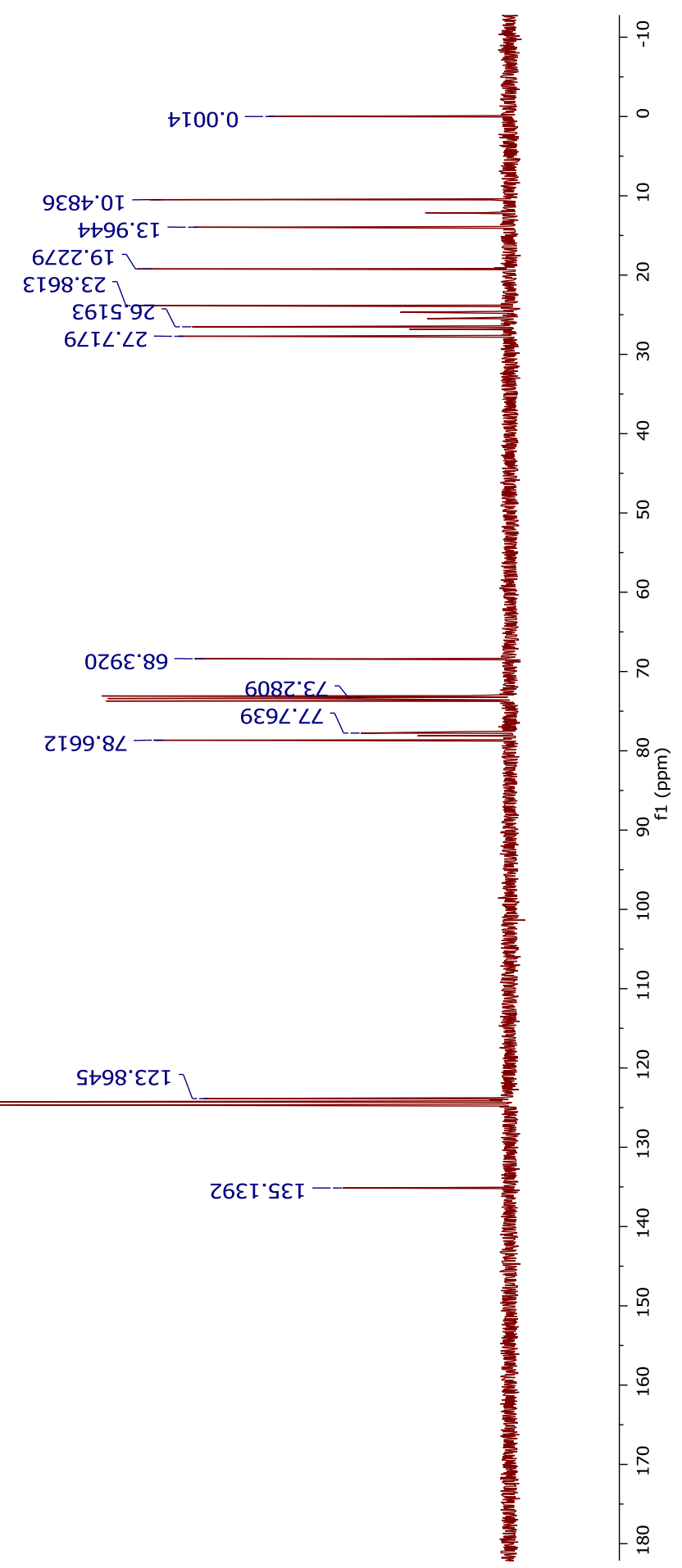




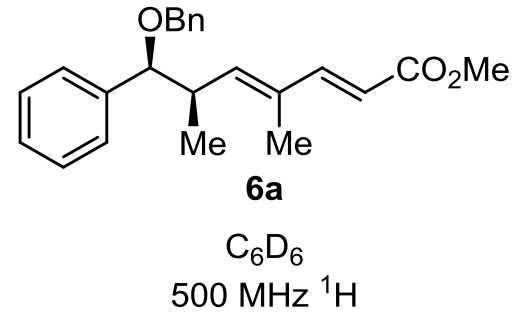

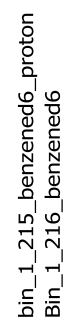

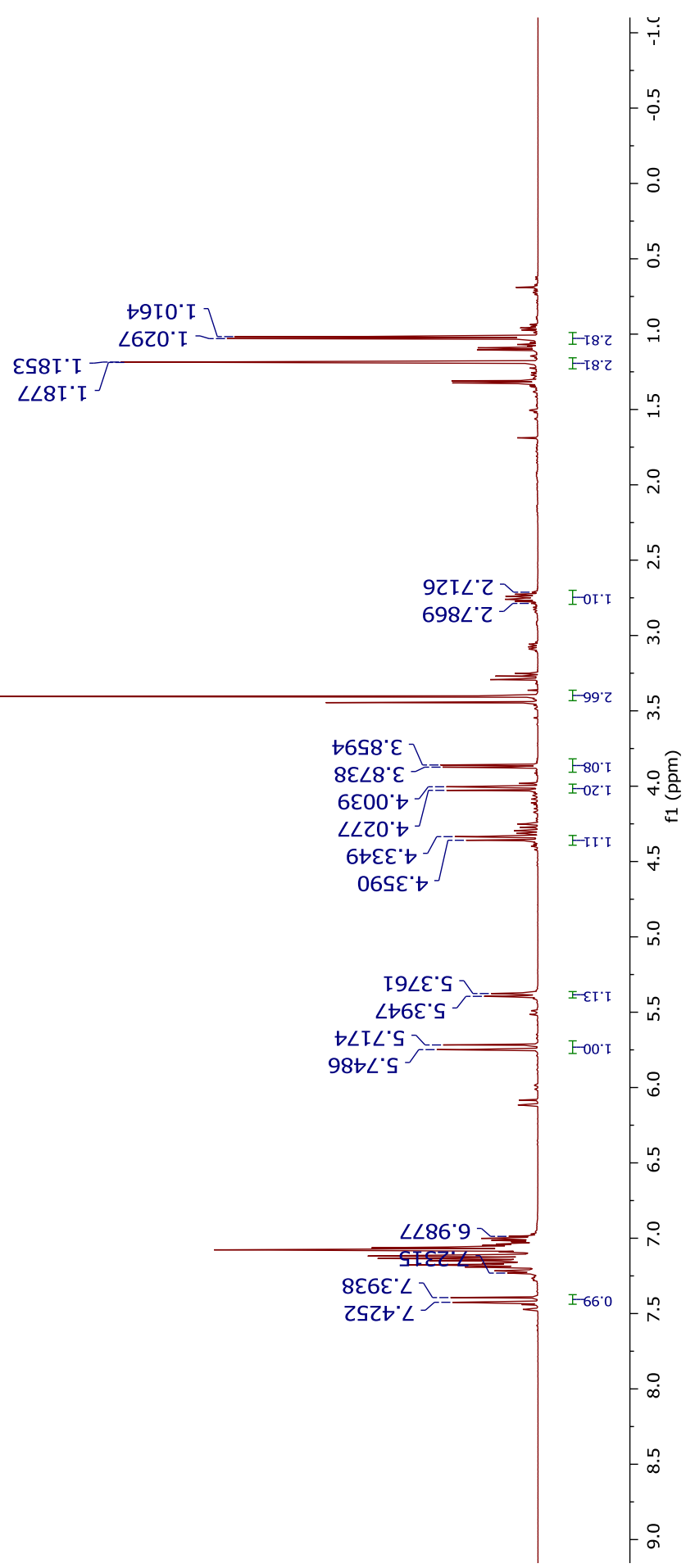




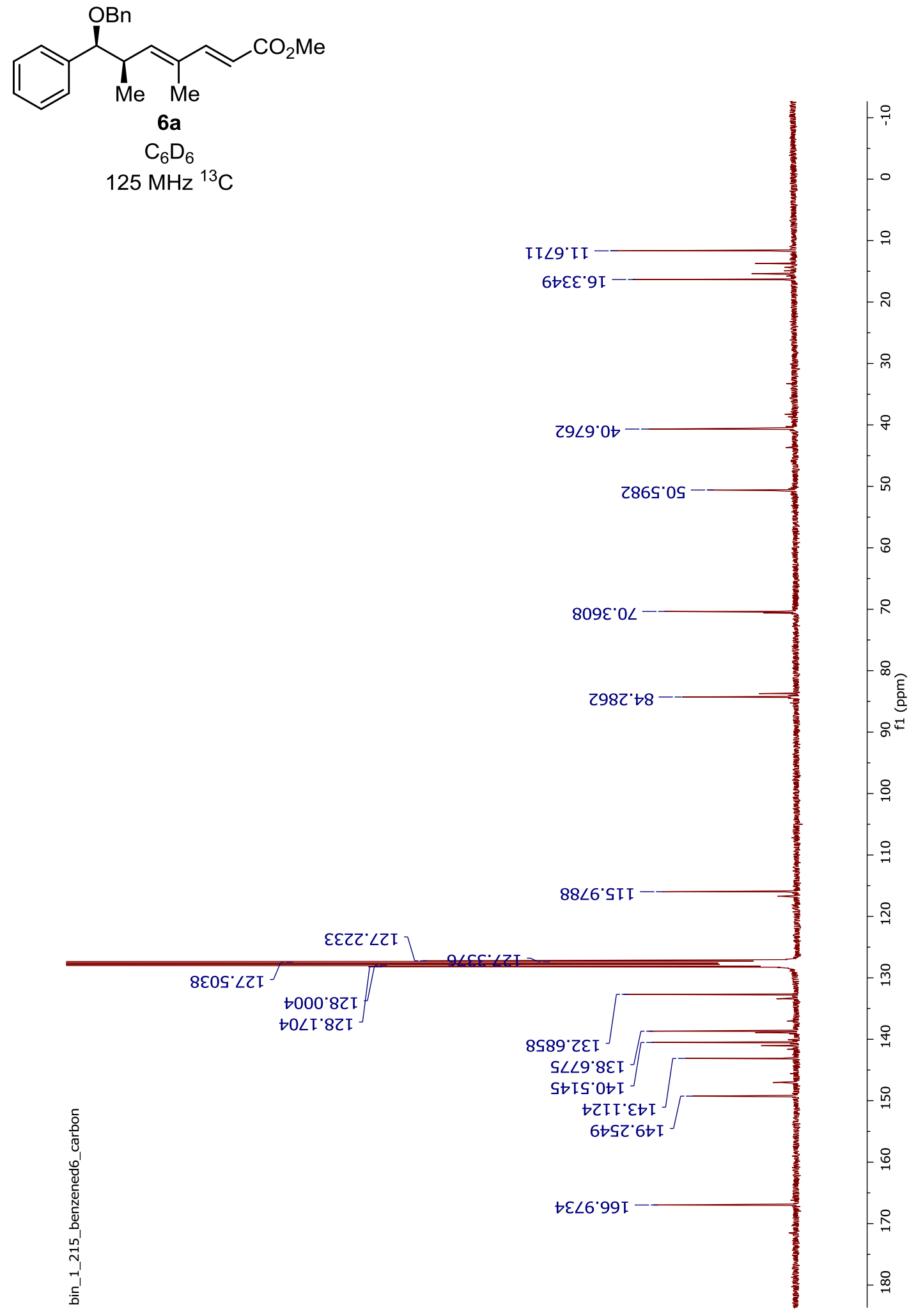



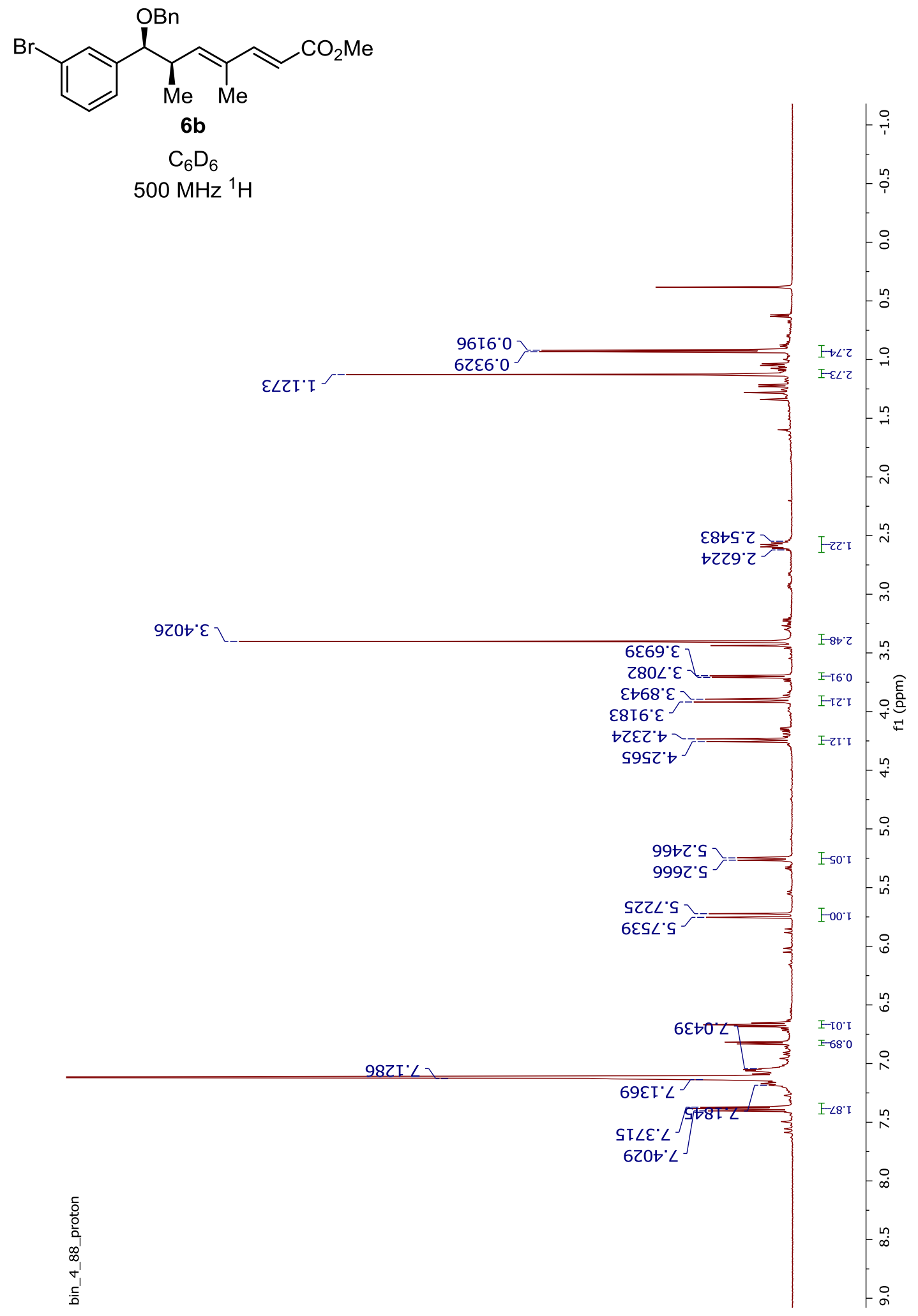

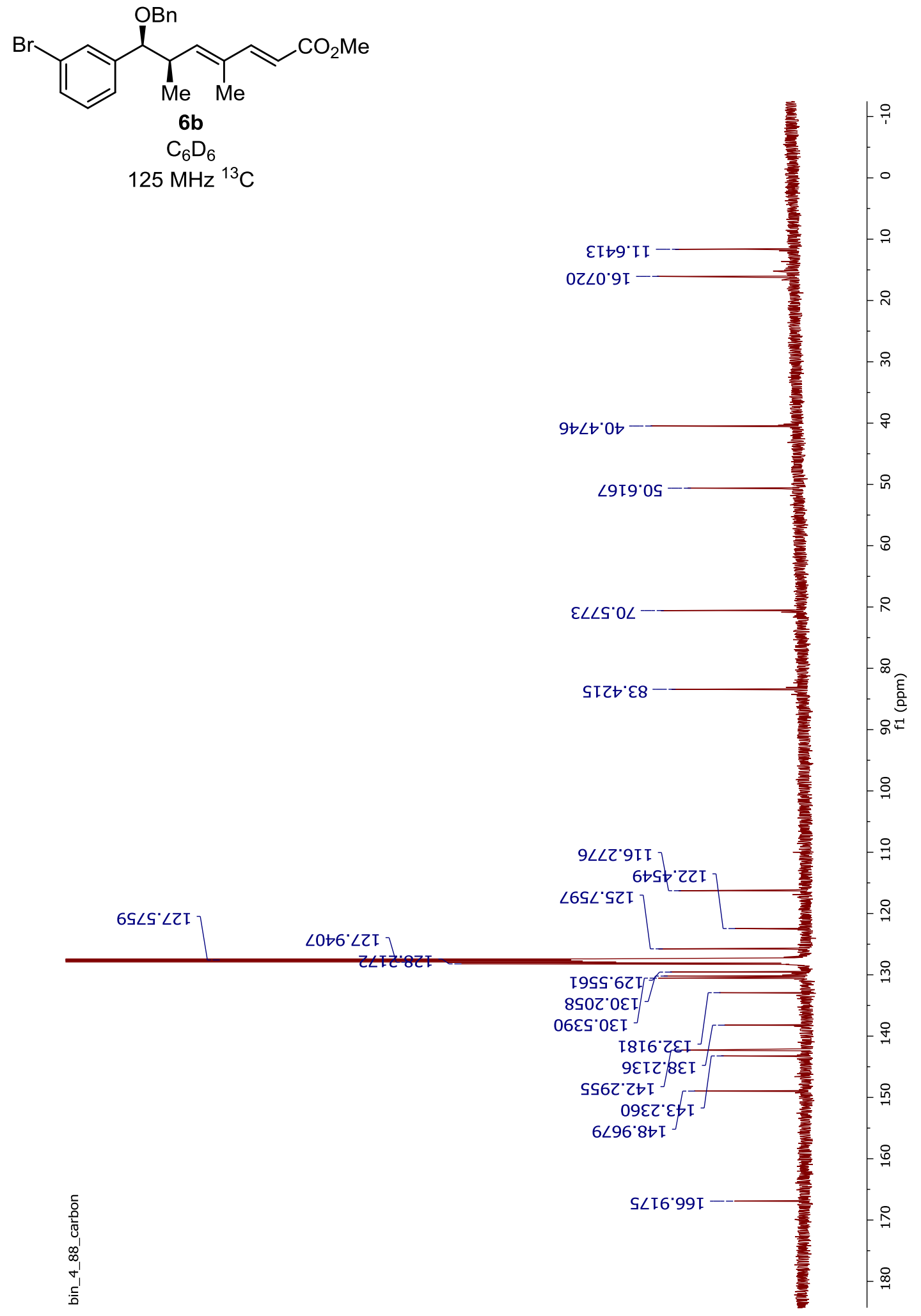


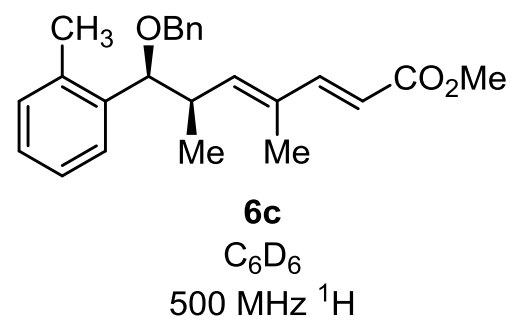

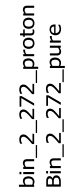

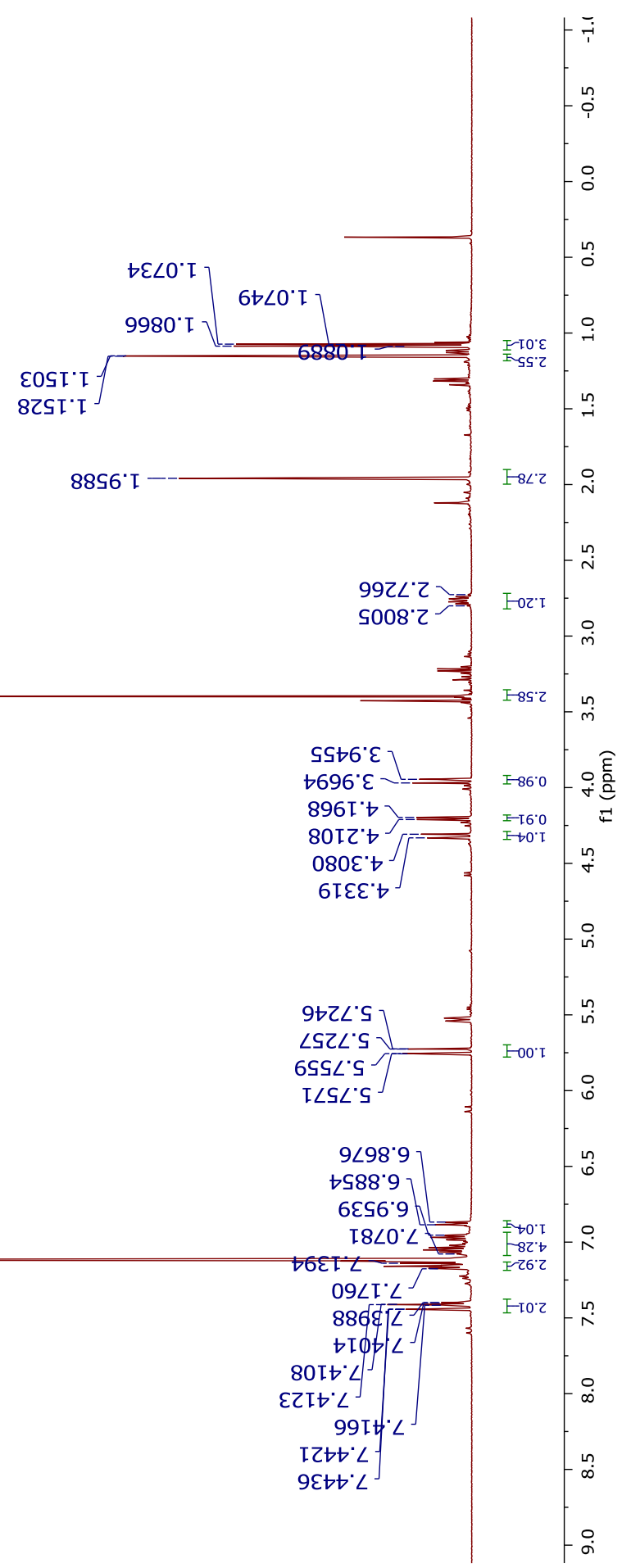




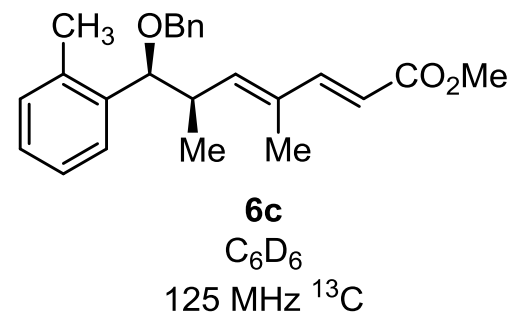

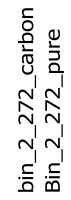

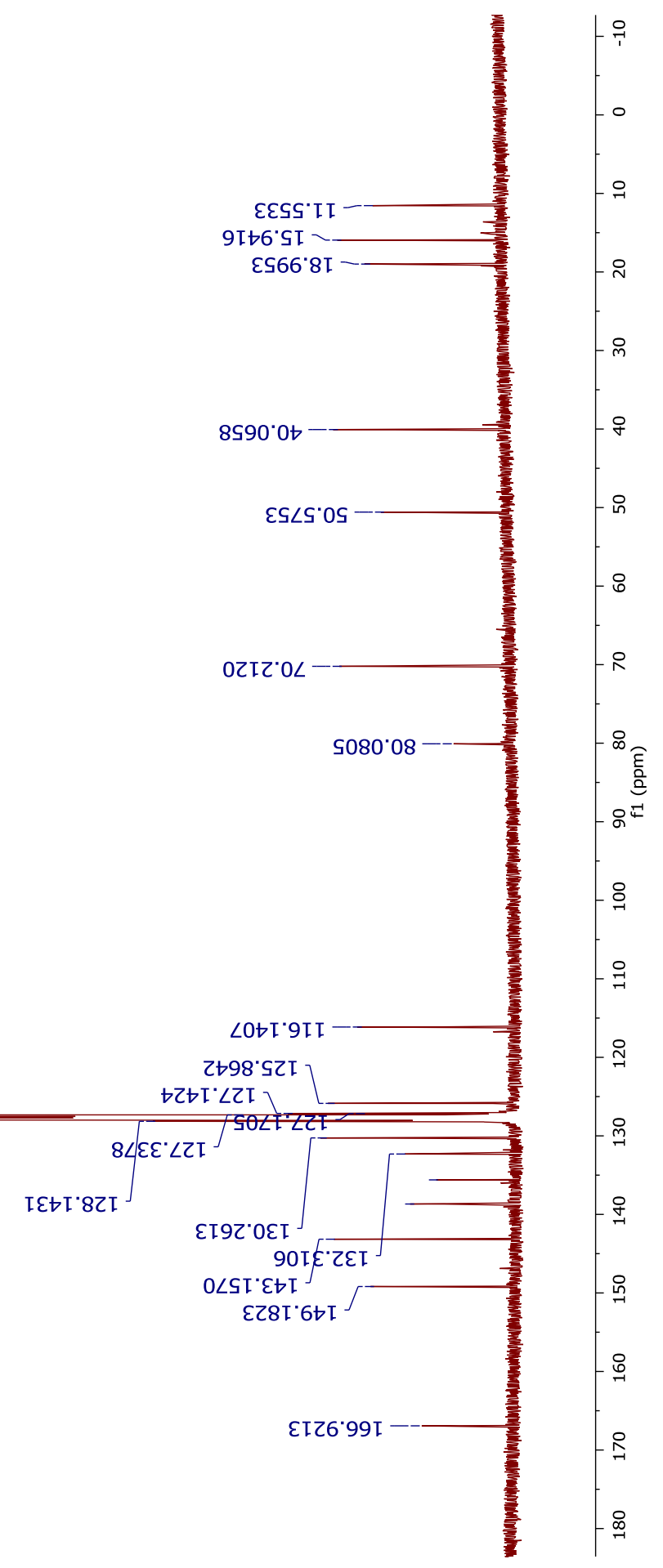




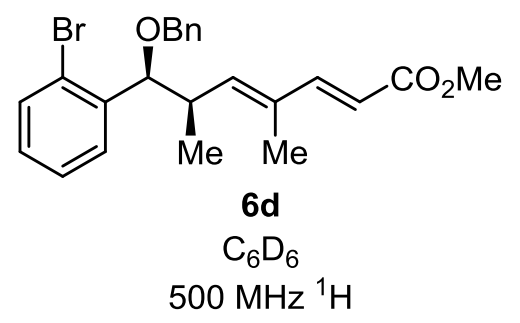

0
0
2
0
0
0
0
0
0
0
0
1
$\vdots$
$\vdots$
$\vdots$

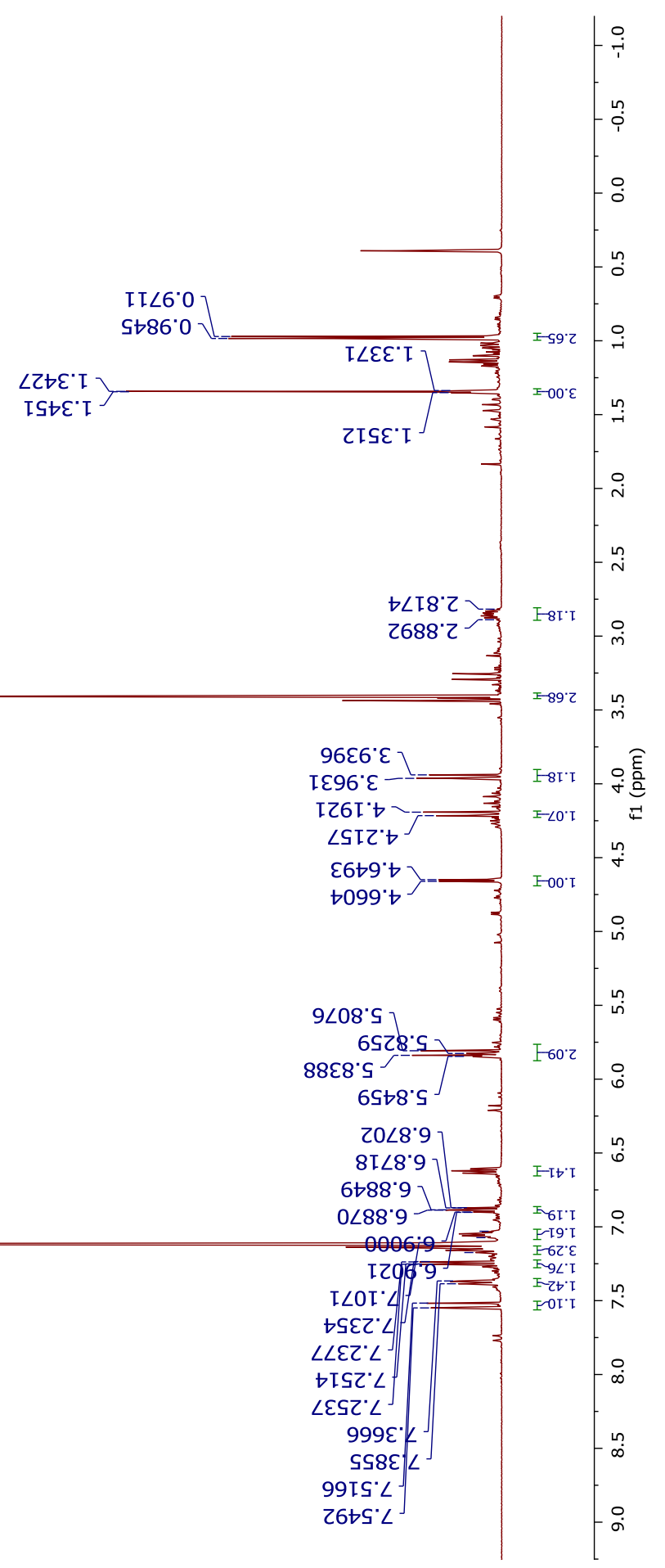




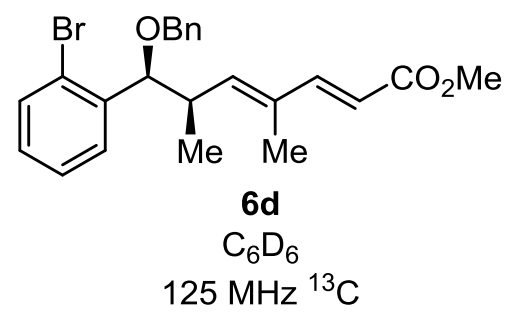

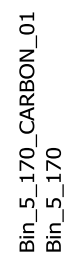

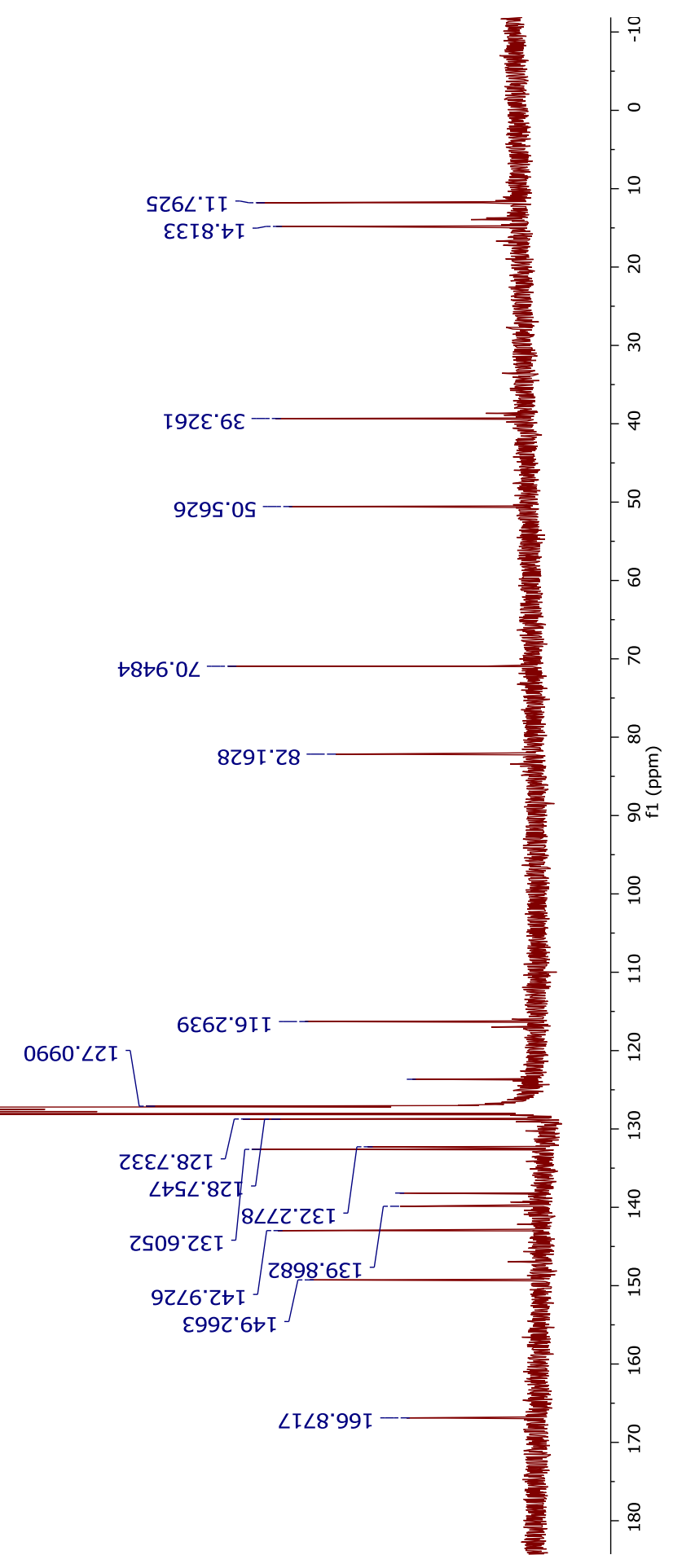


<smiles>COC(=O)C=CC(C)=CC(C)[C@H](OCc1ccccc1)c1ccc(F)cc1</smiles>

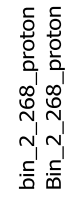

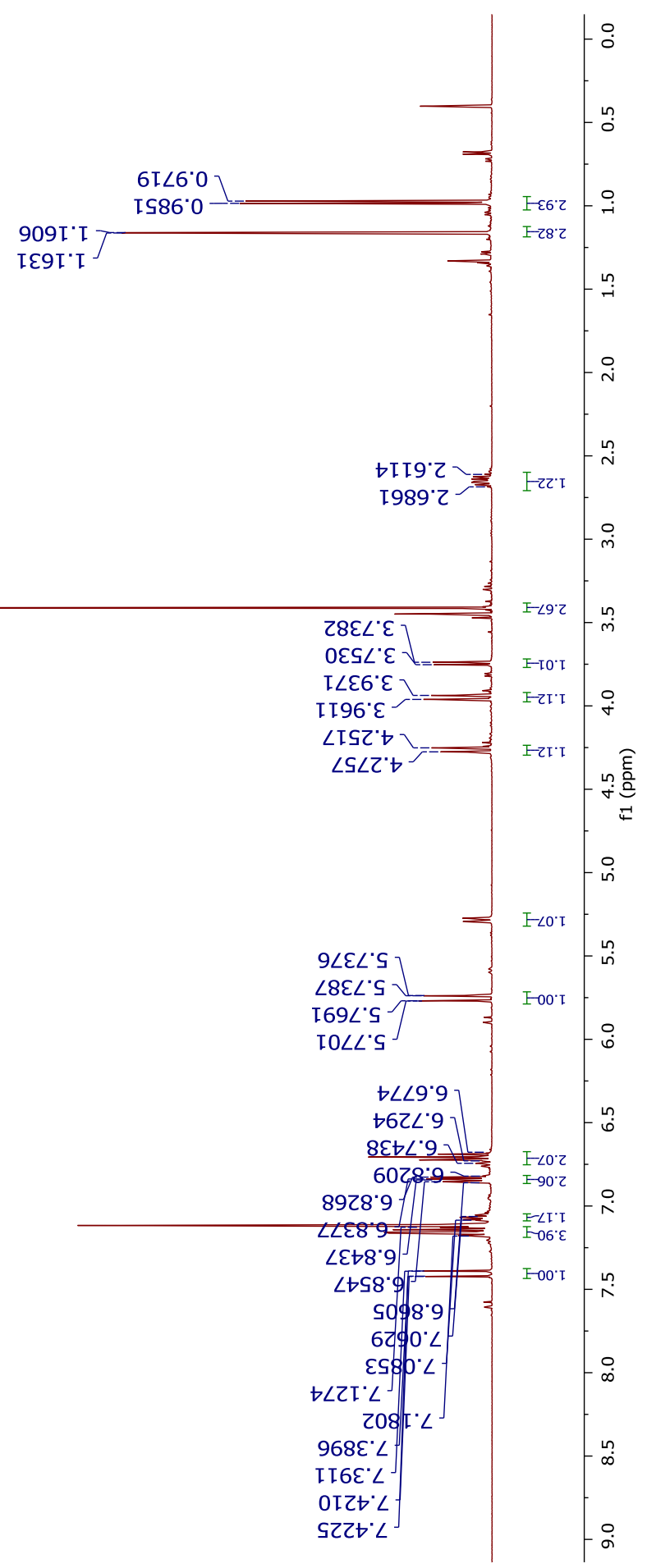


<smiles>COC(=O)C=CC(C)=CC(C)[C@H](OCc1ccccc1)c1ccc(F)cc1</smiles>

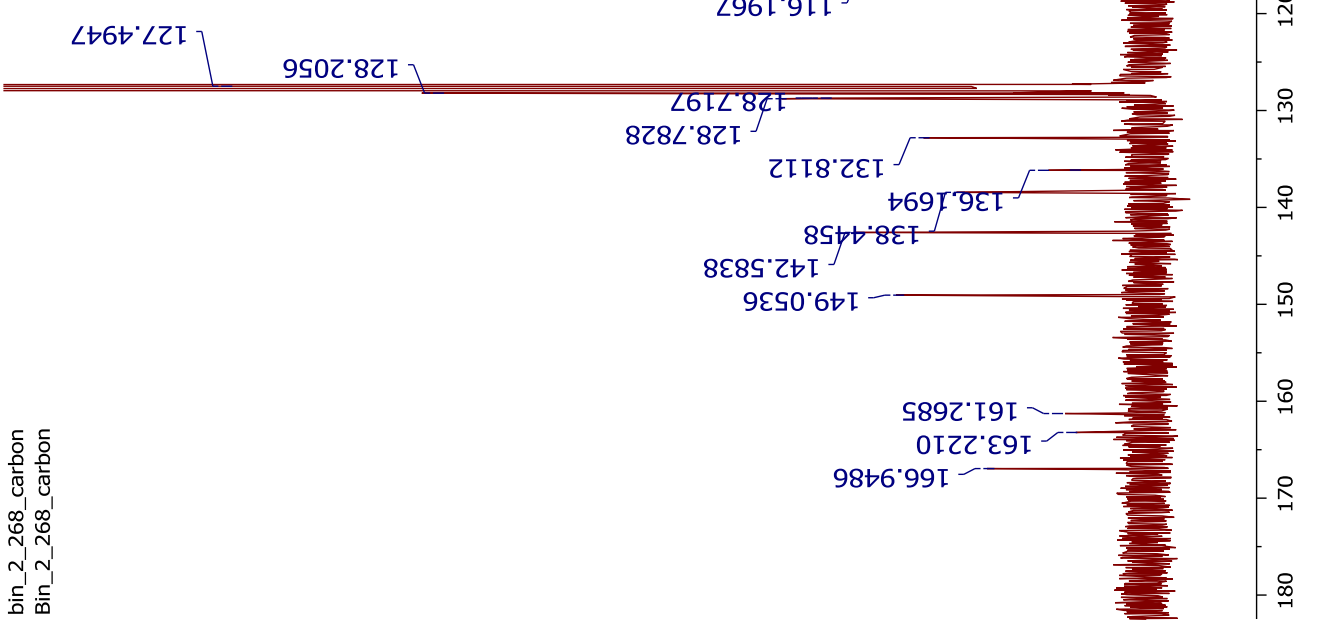



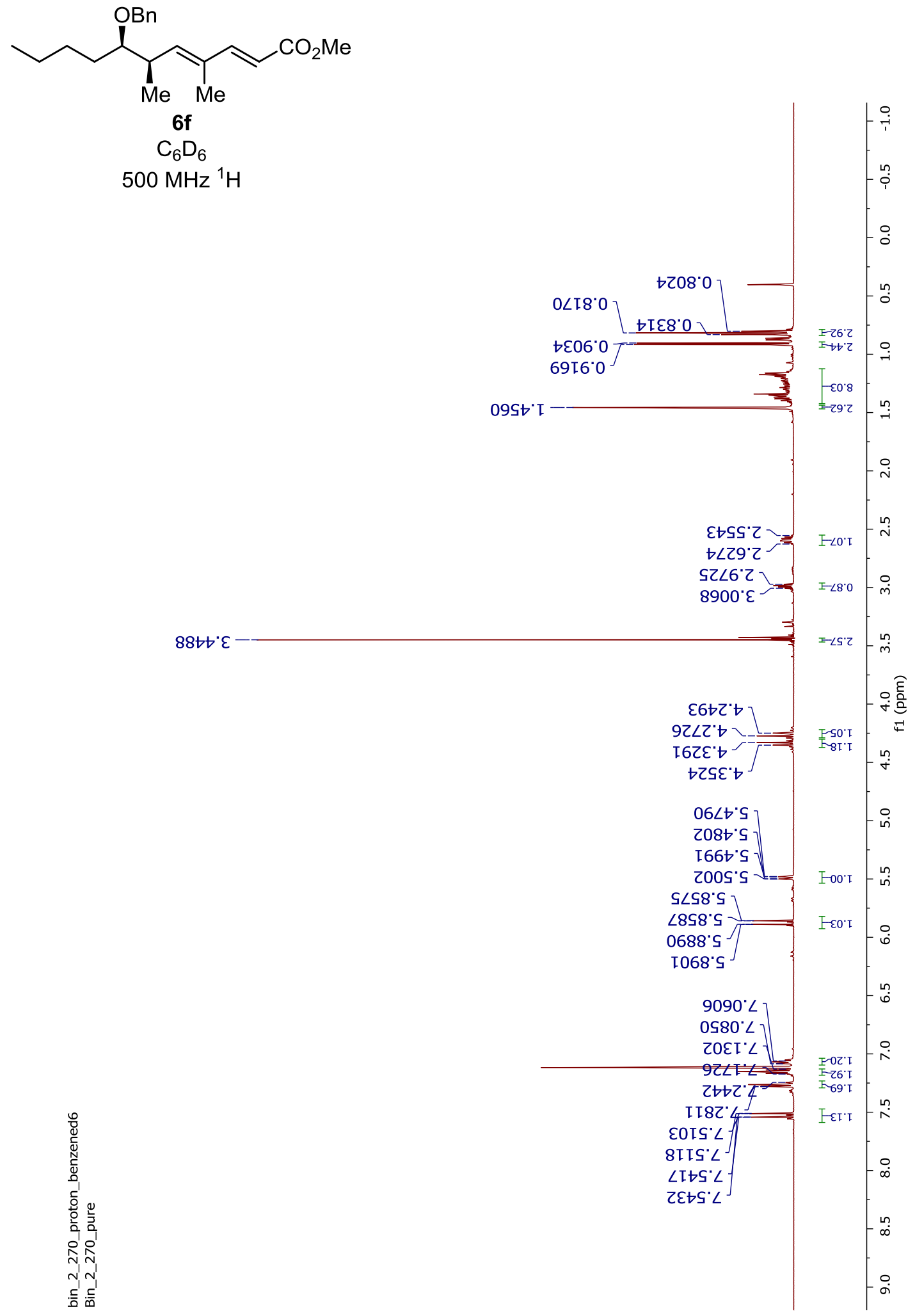


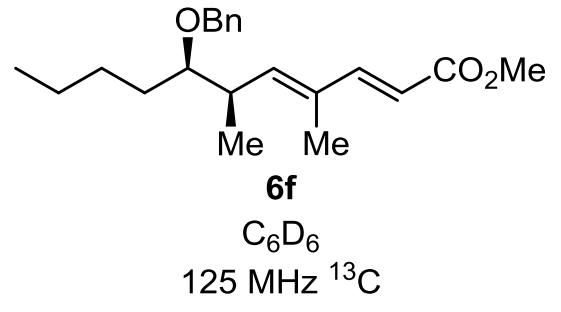

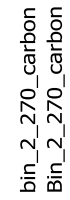

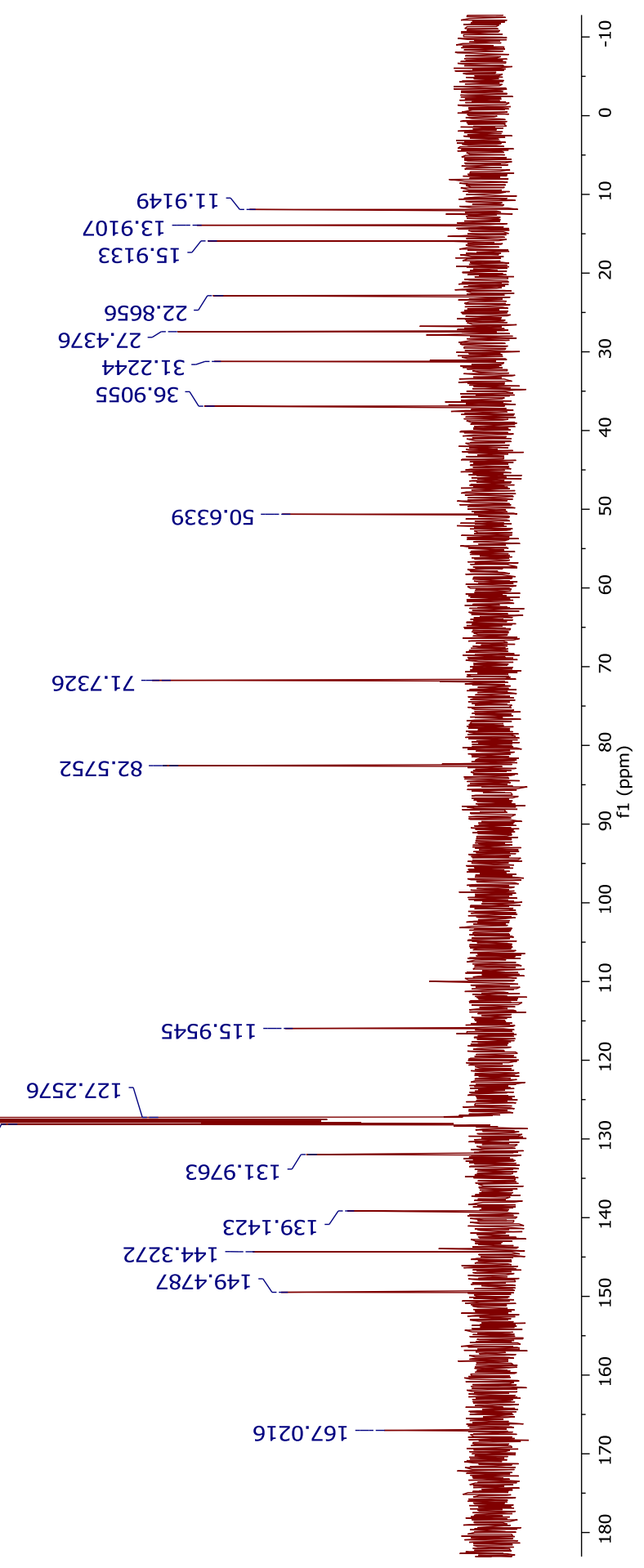



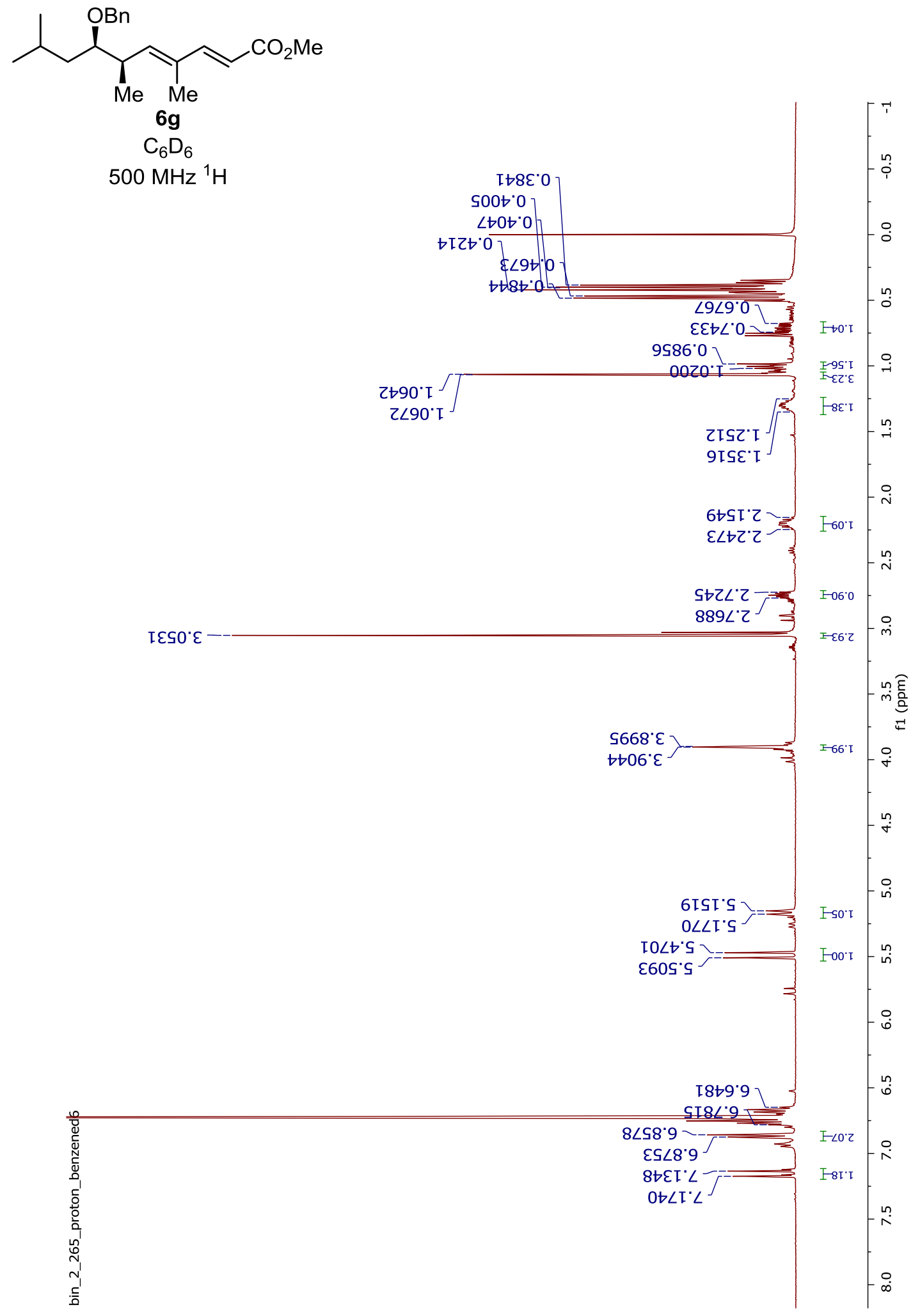


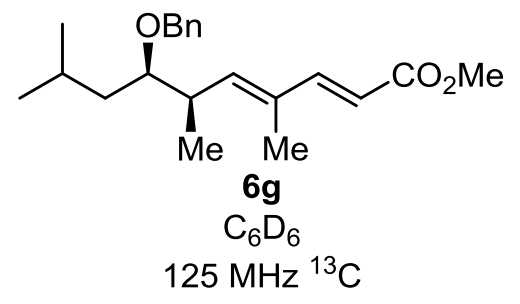

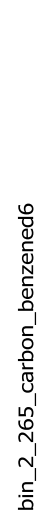

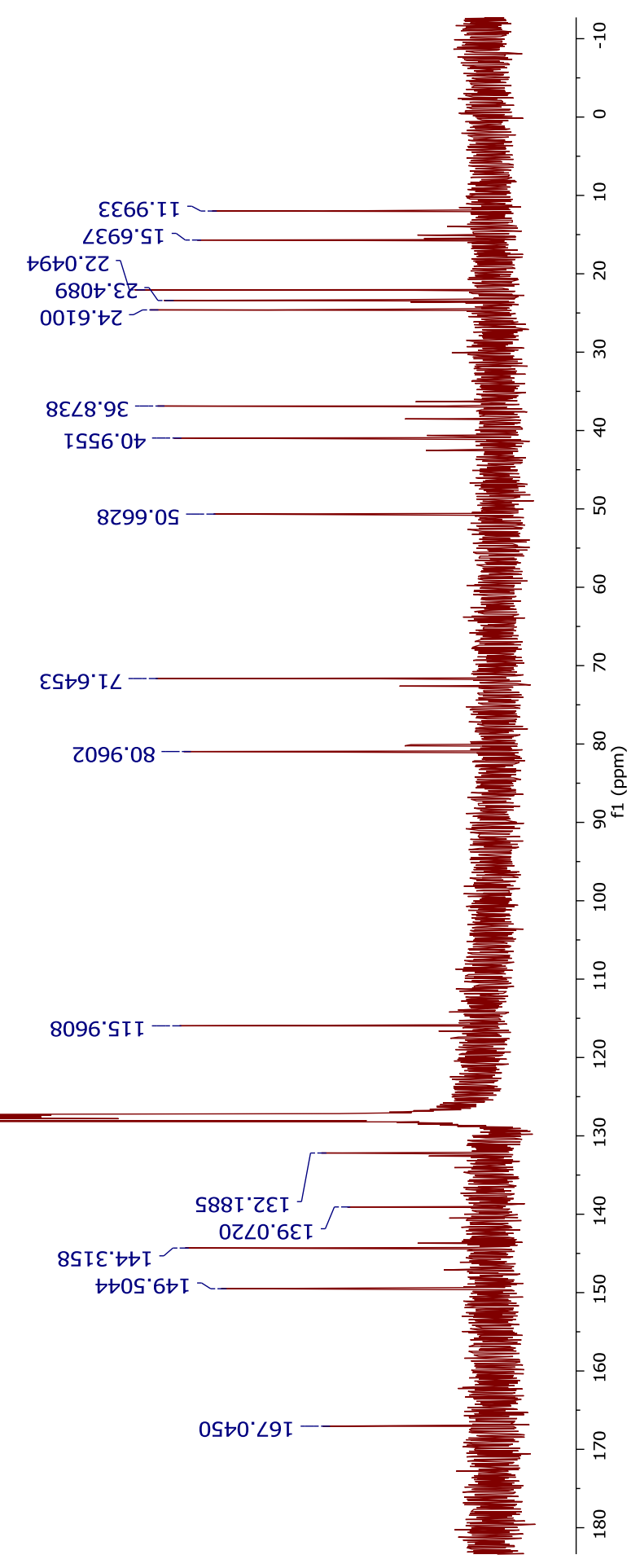




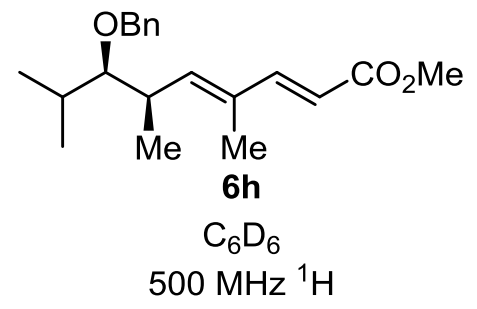

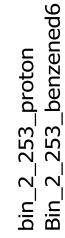

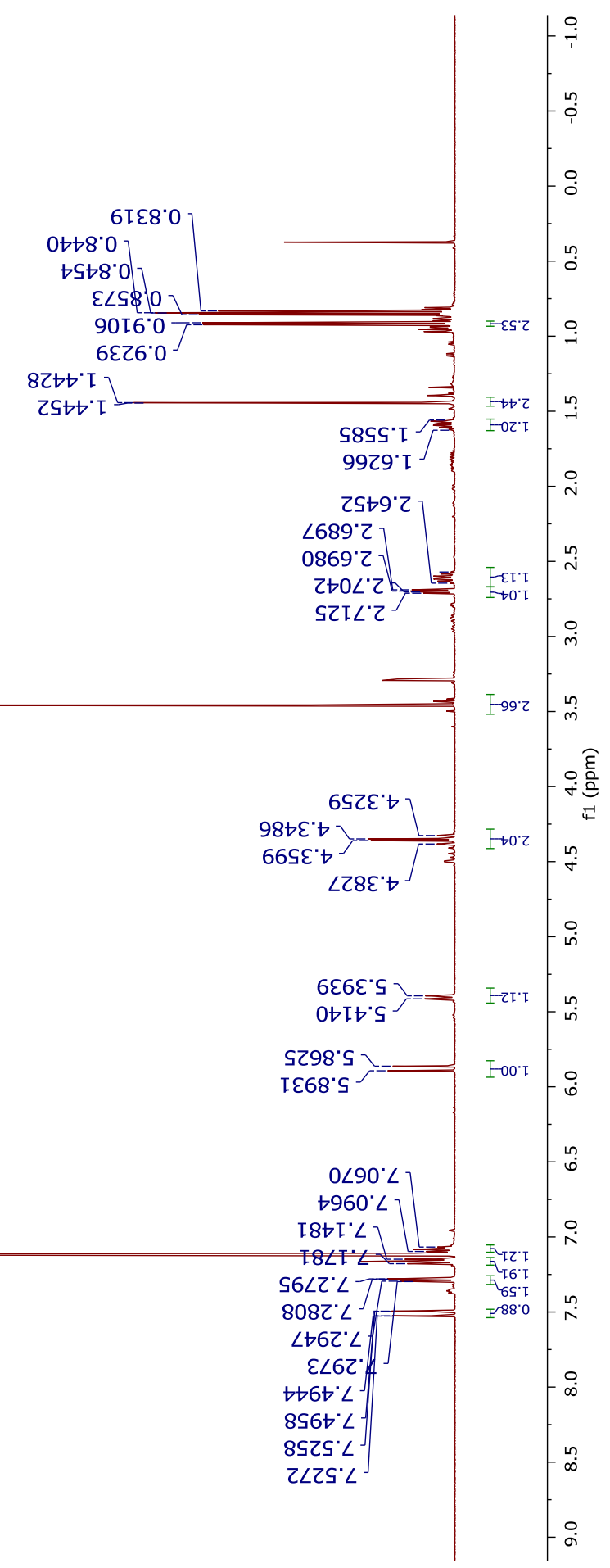




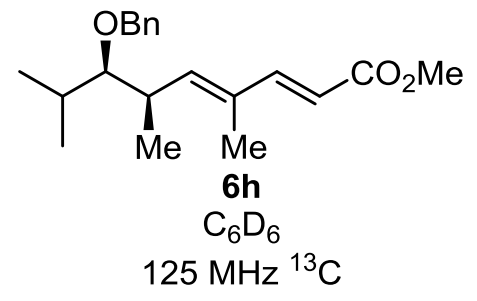

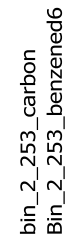

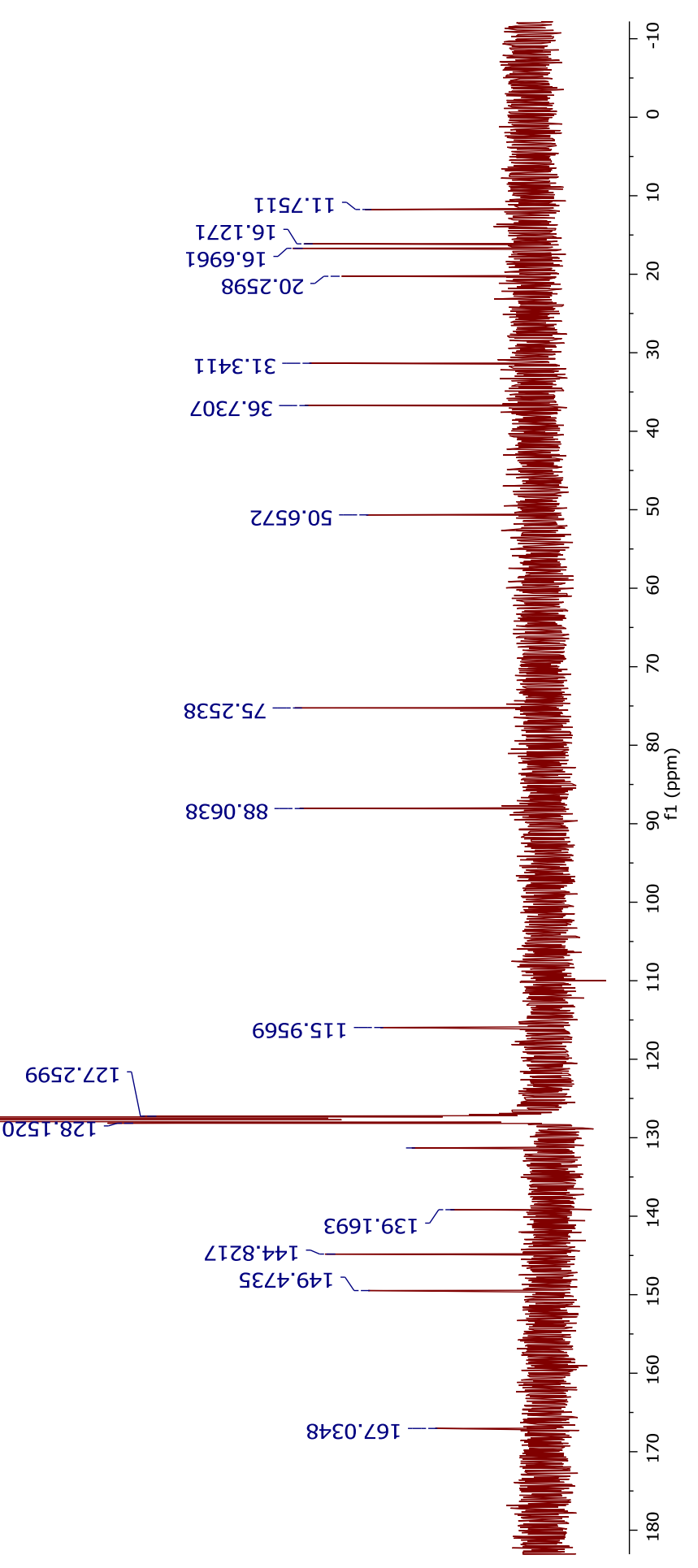




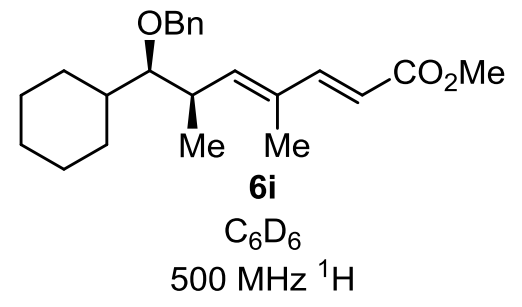

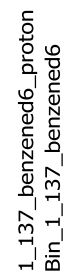

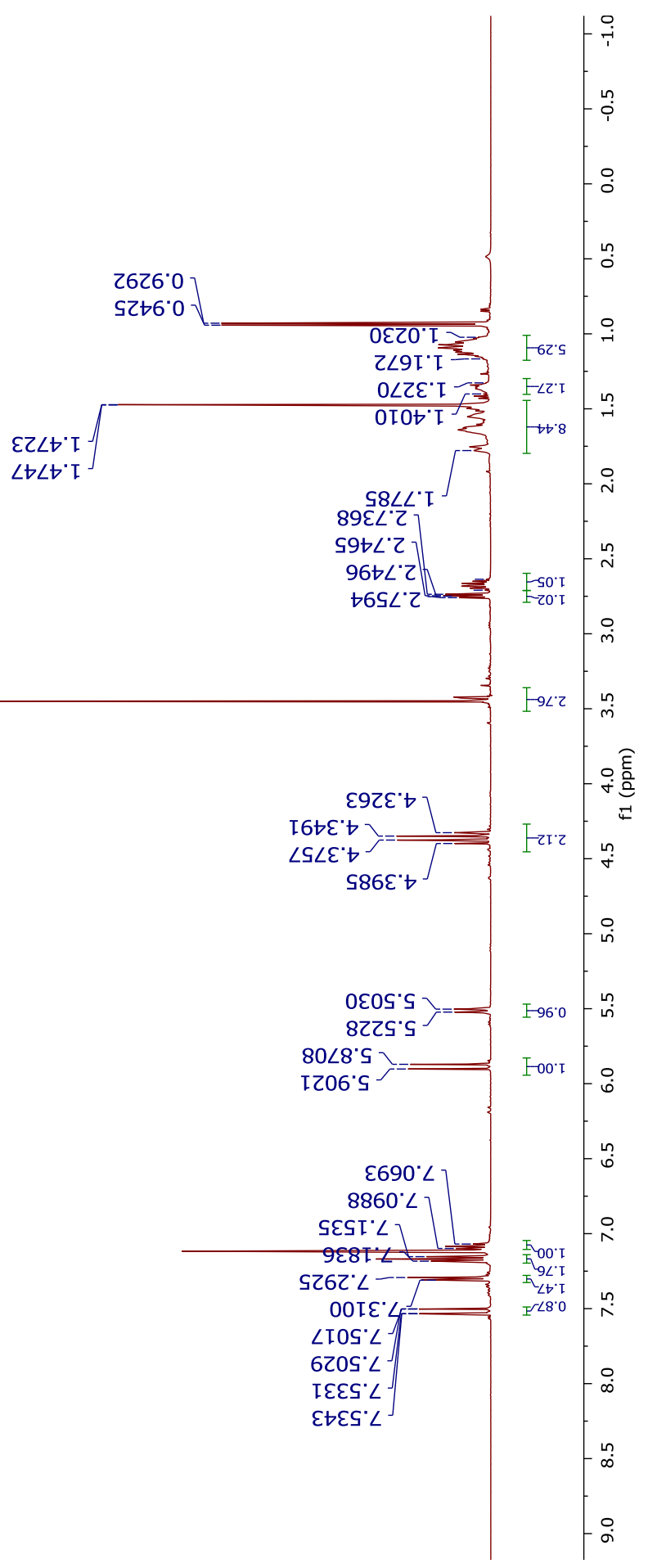




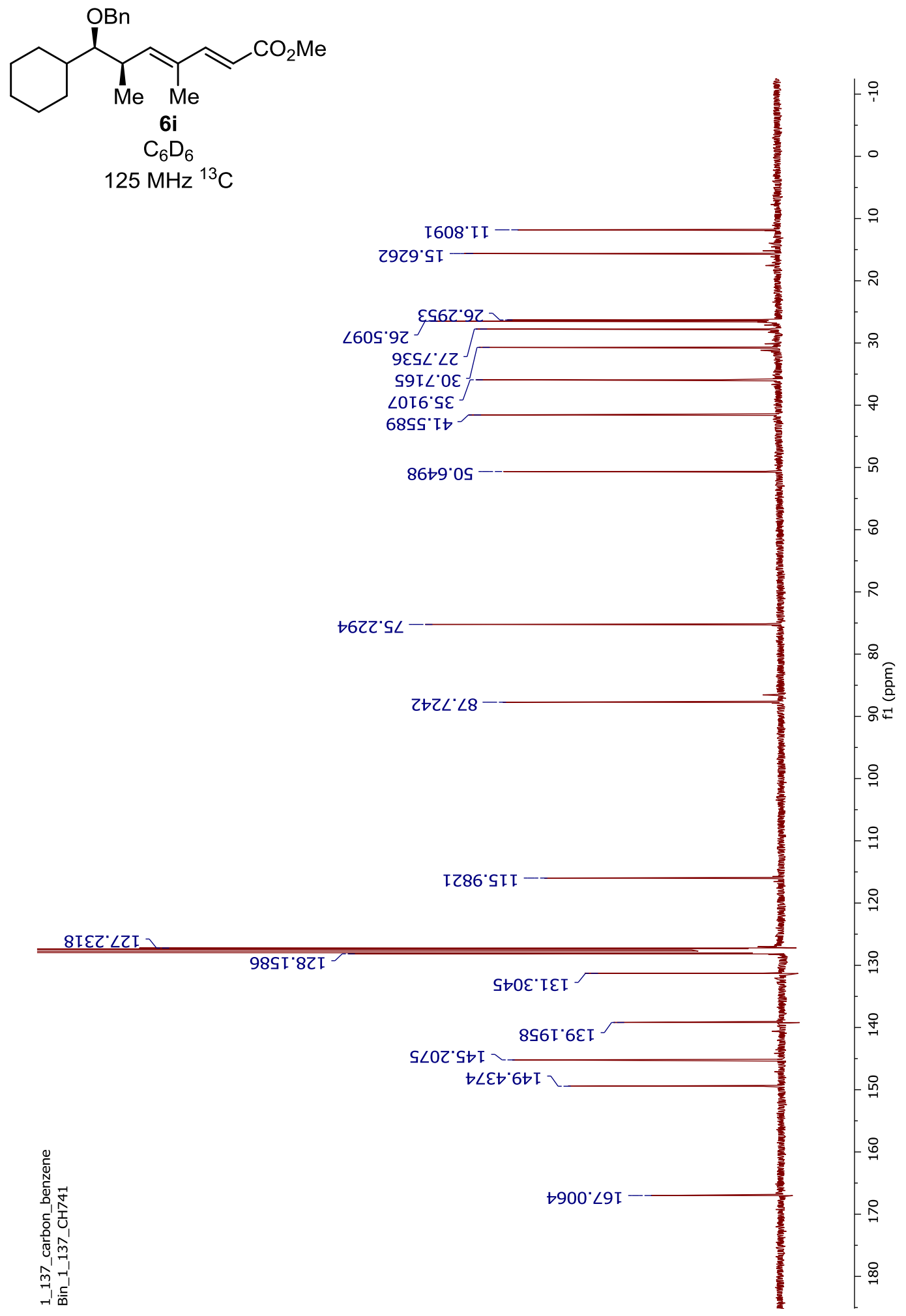



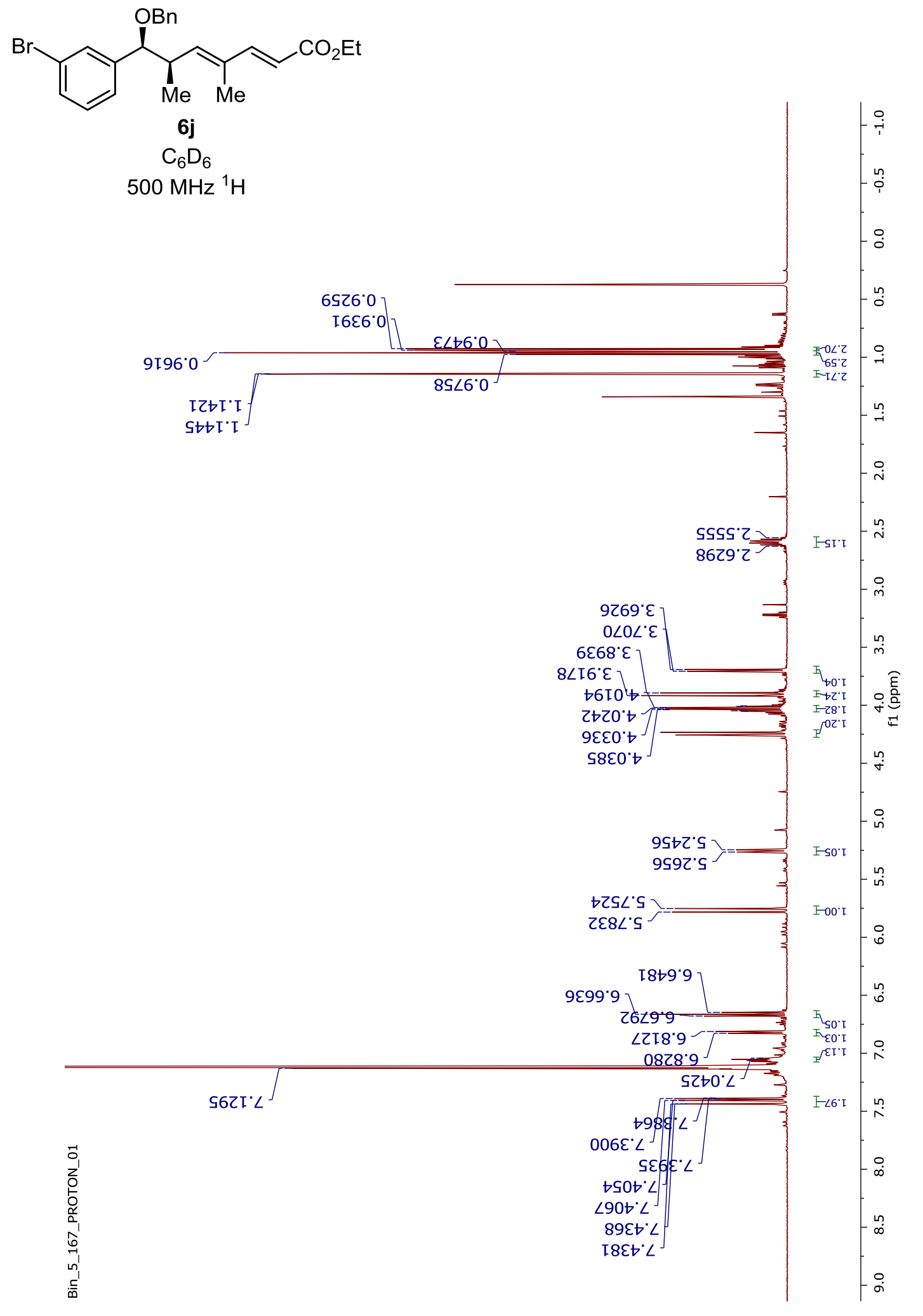

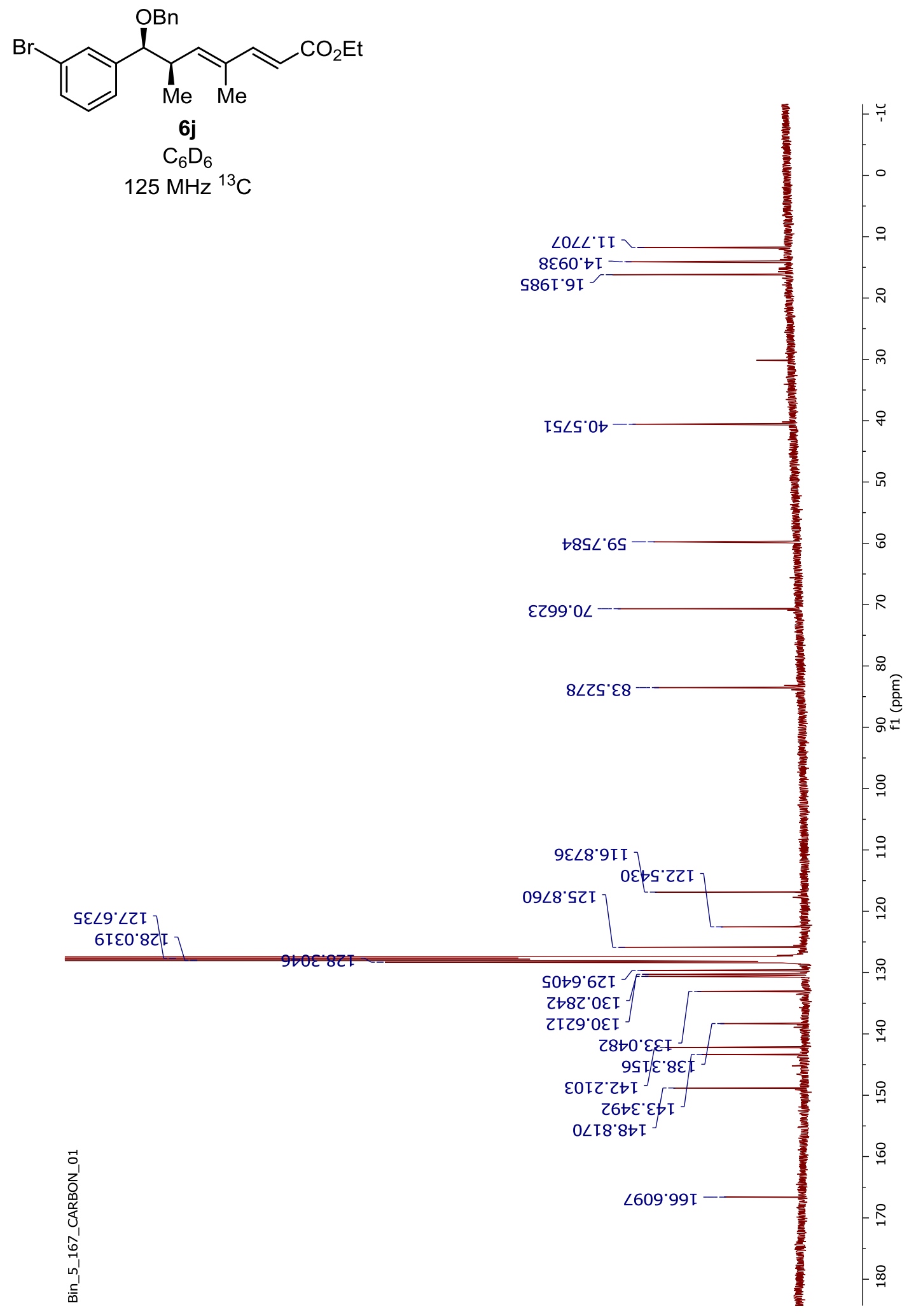

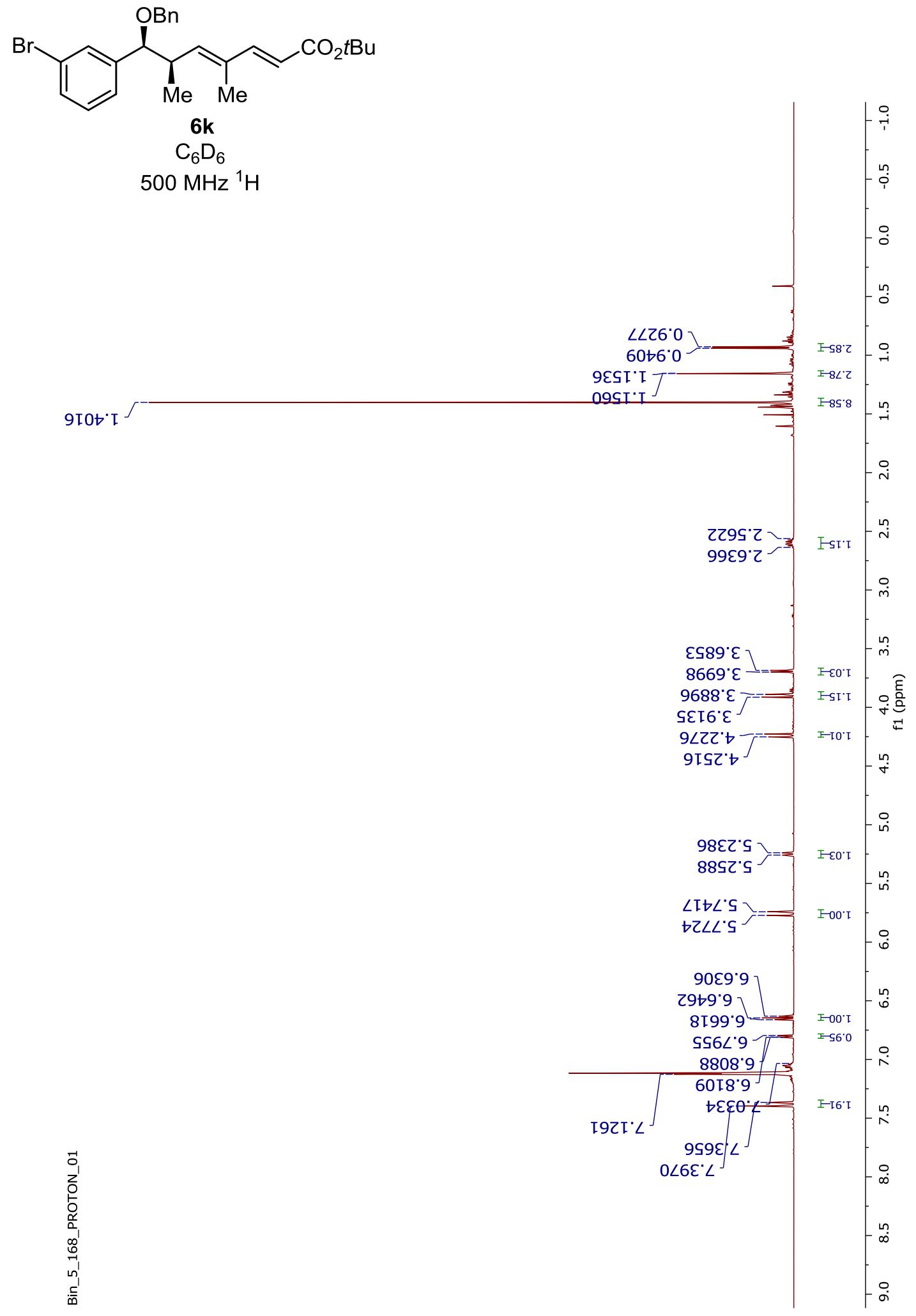

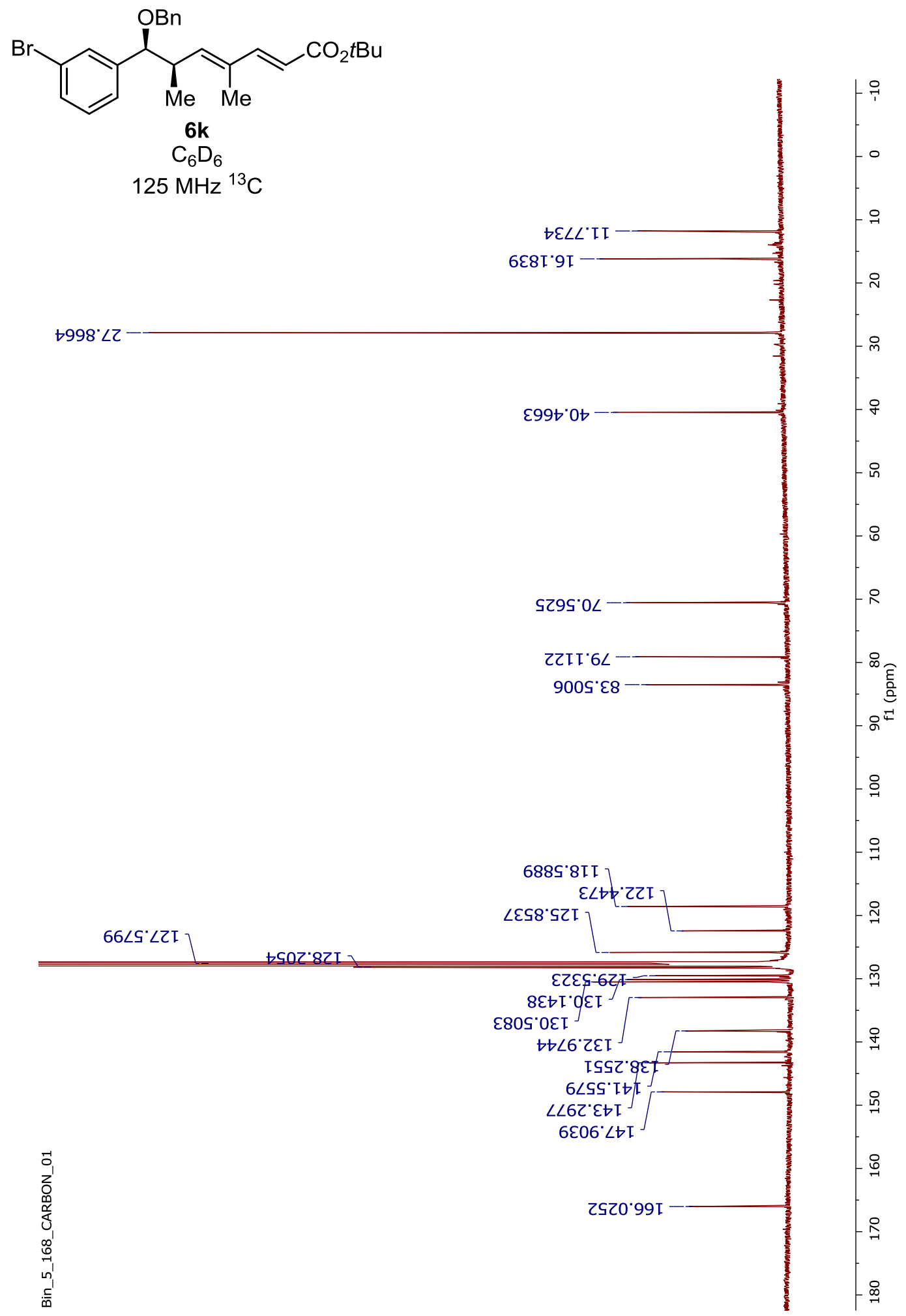


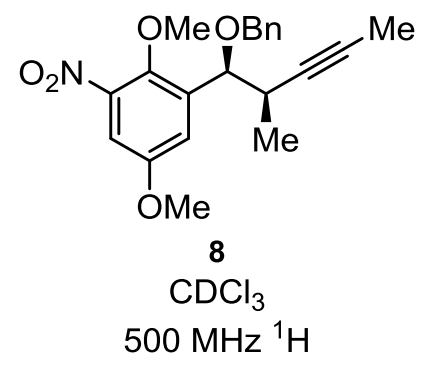

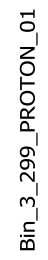

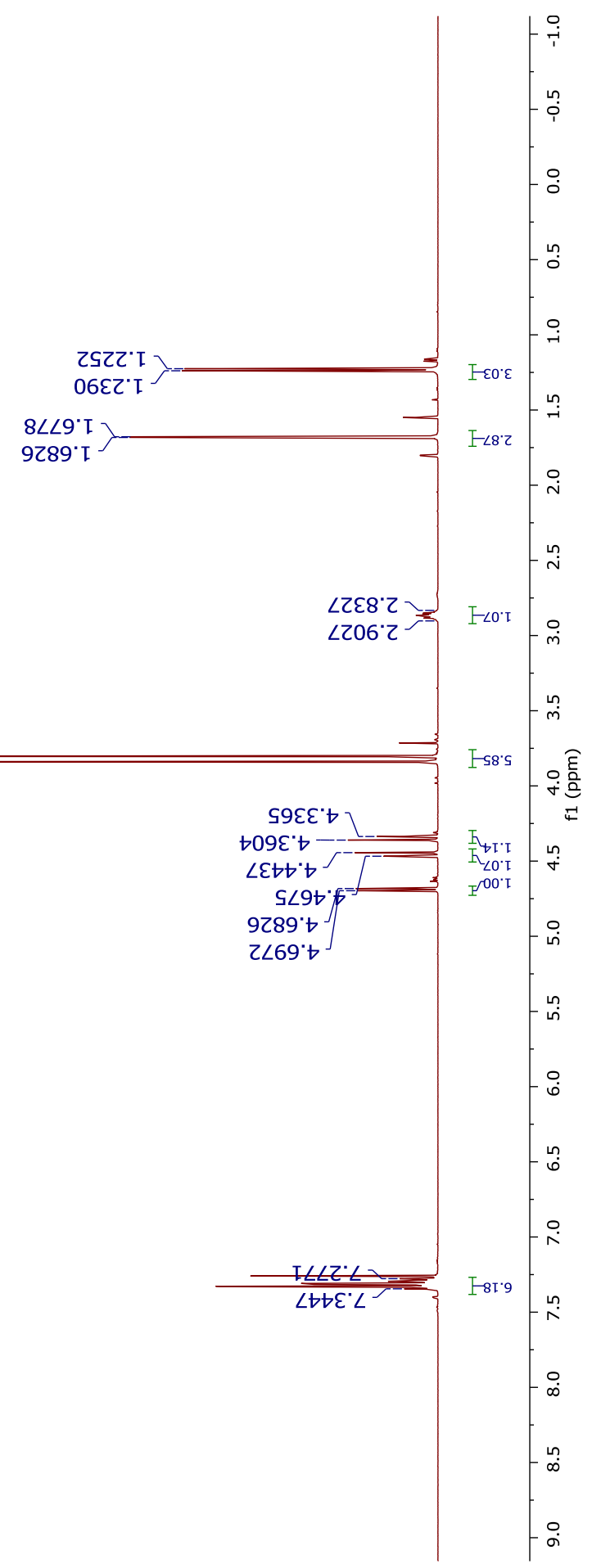



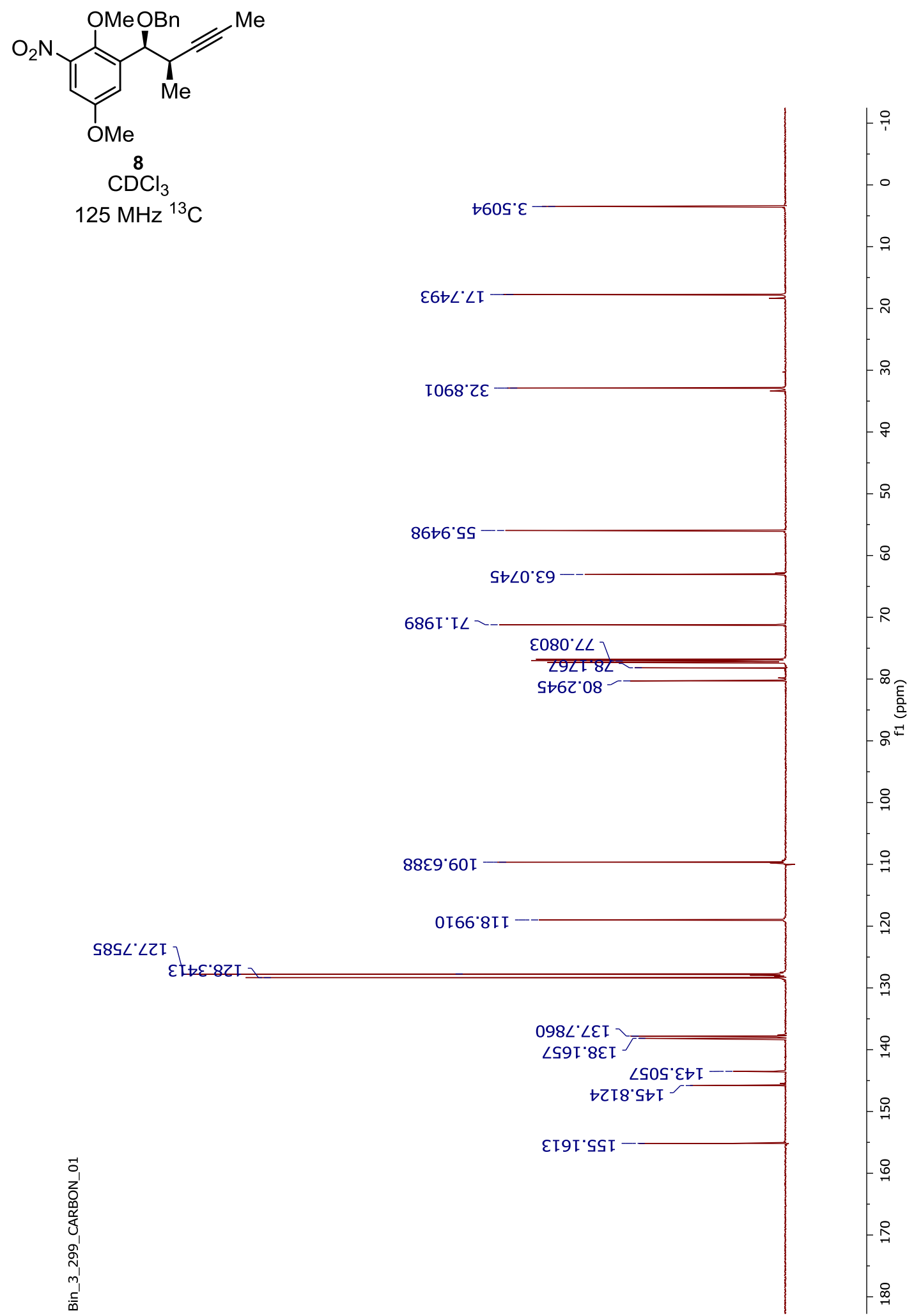

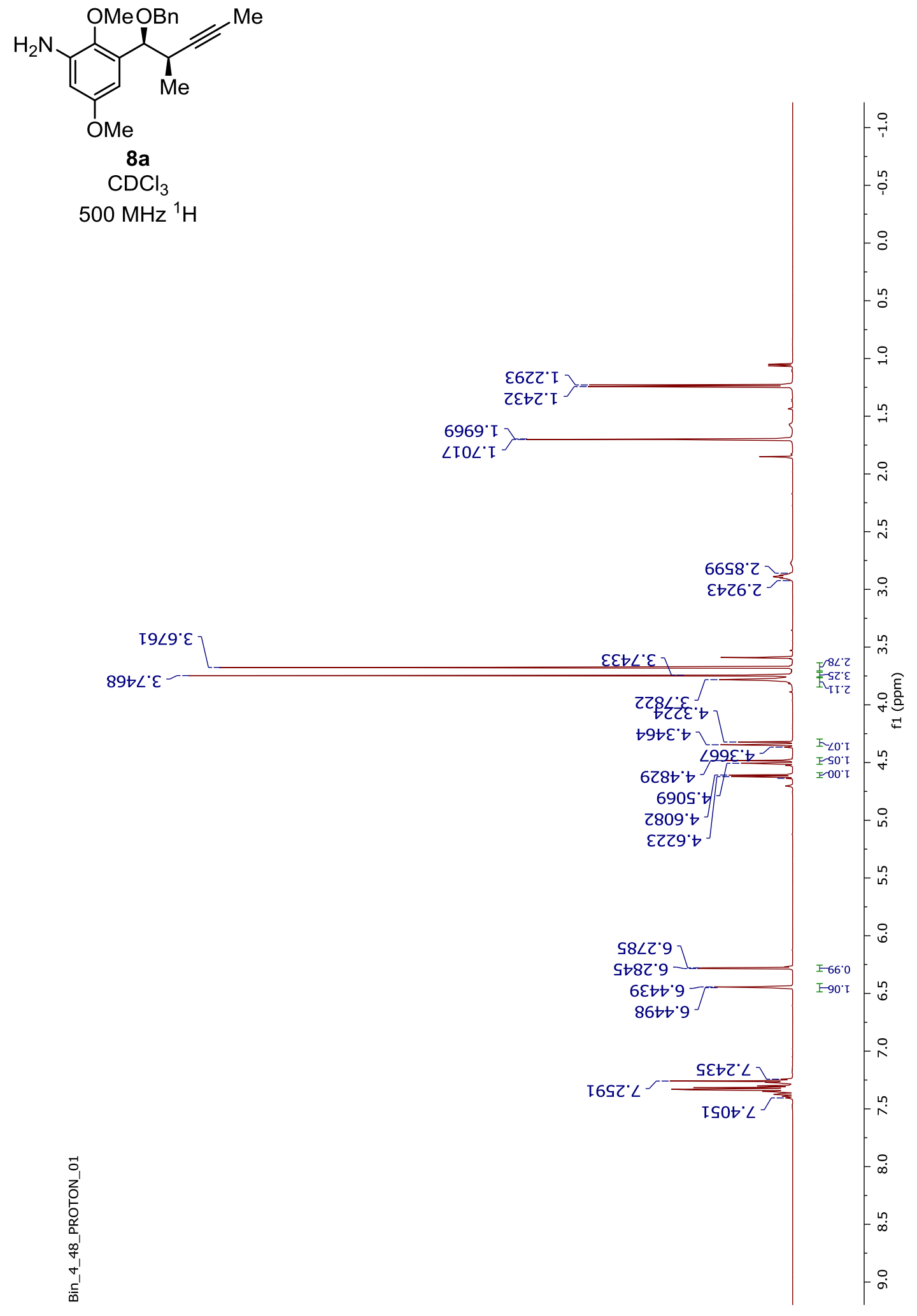

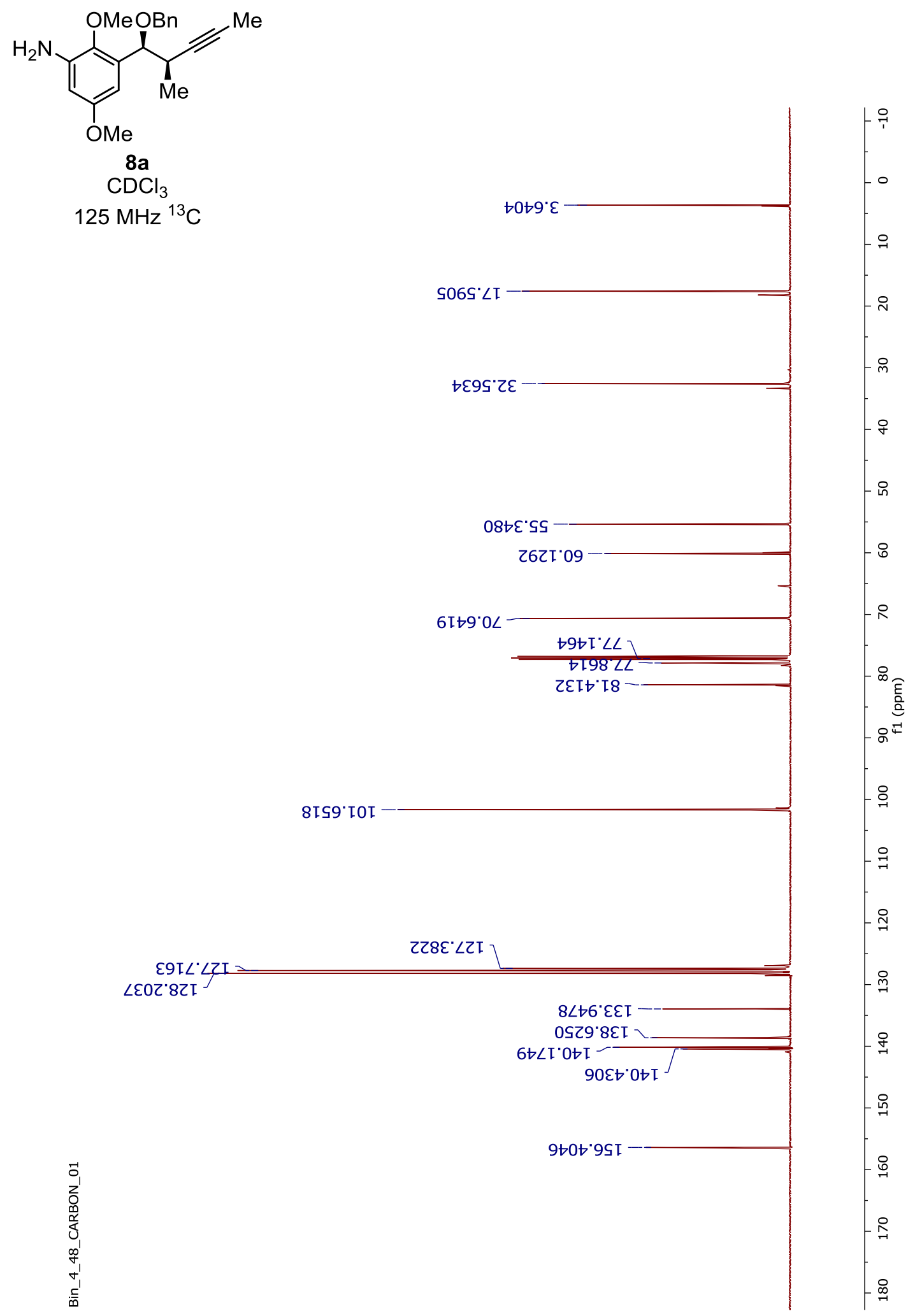


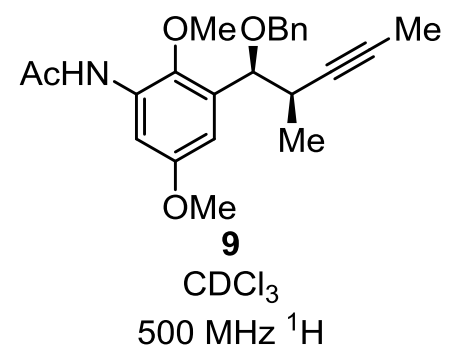

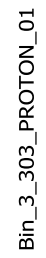

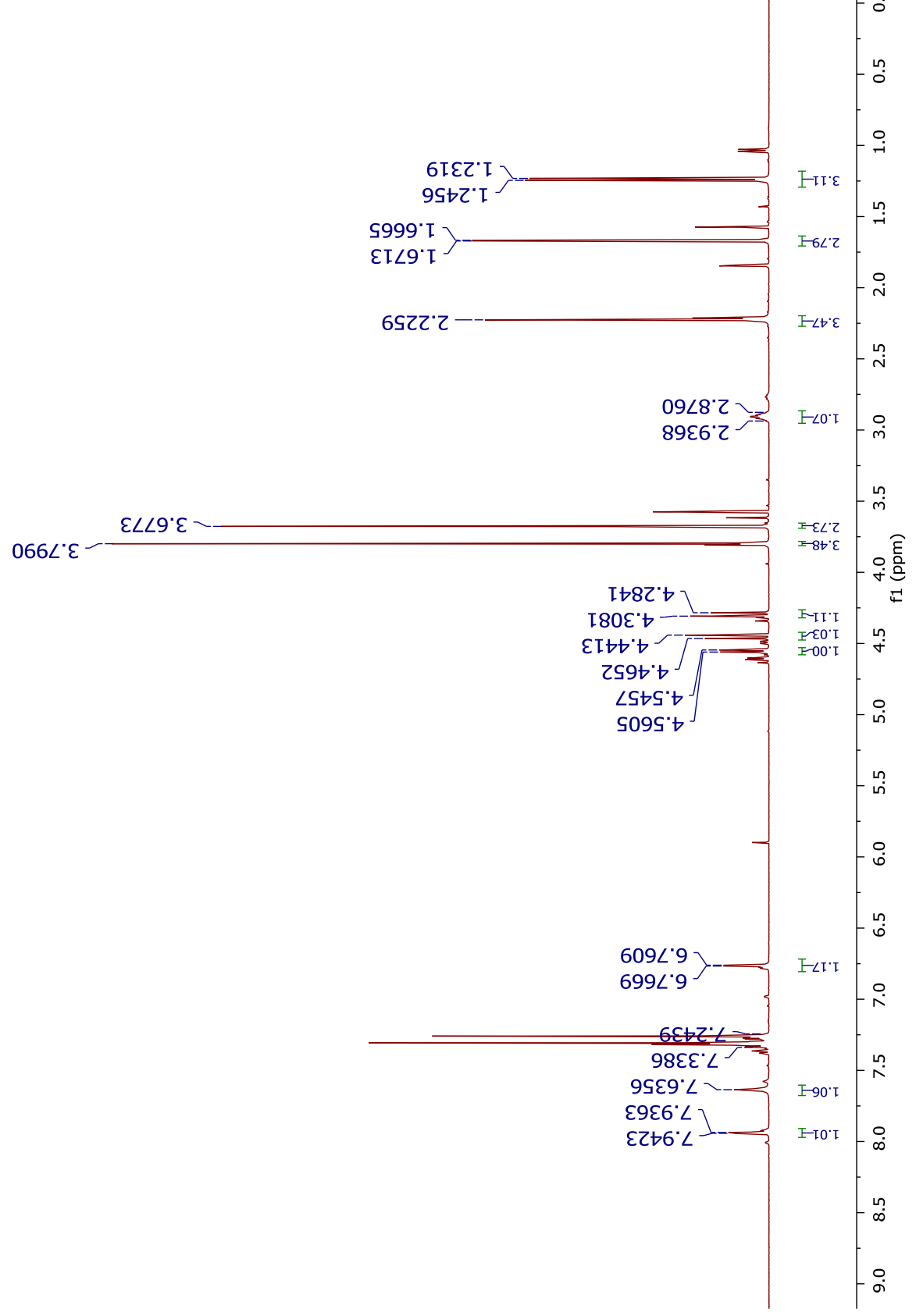




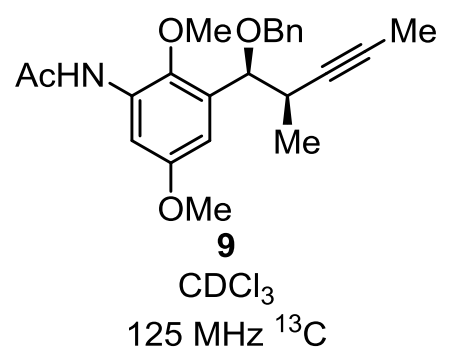

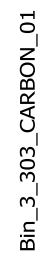

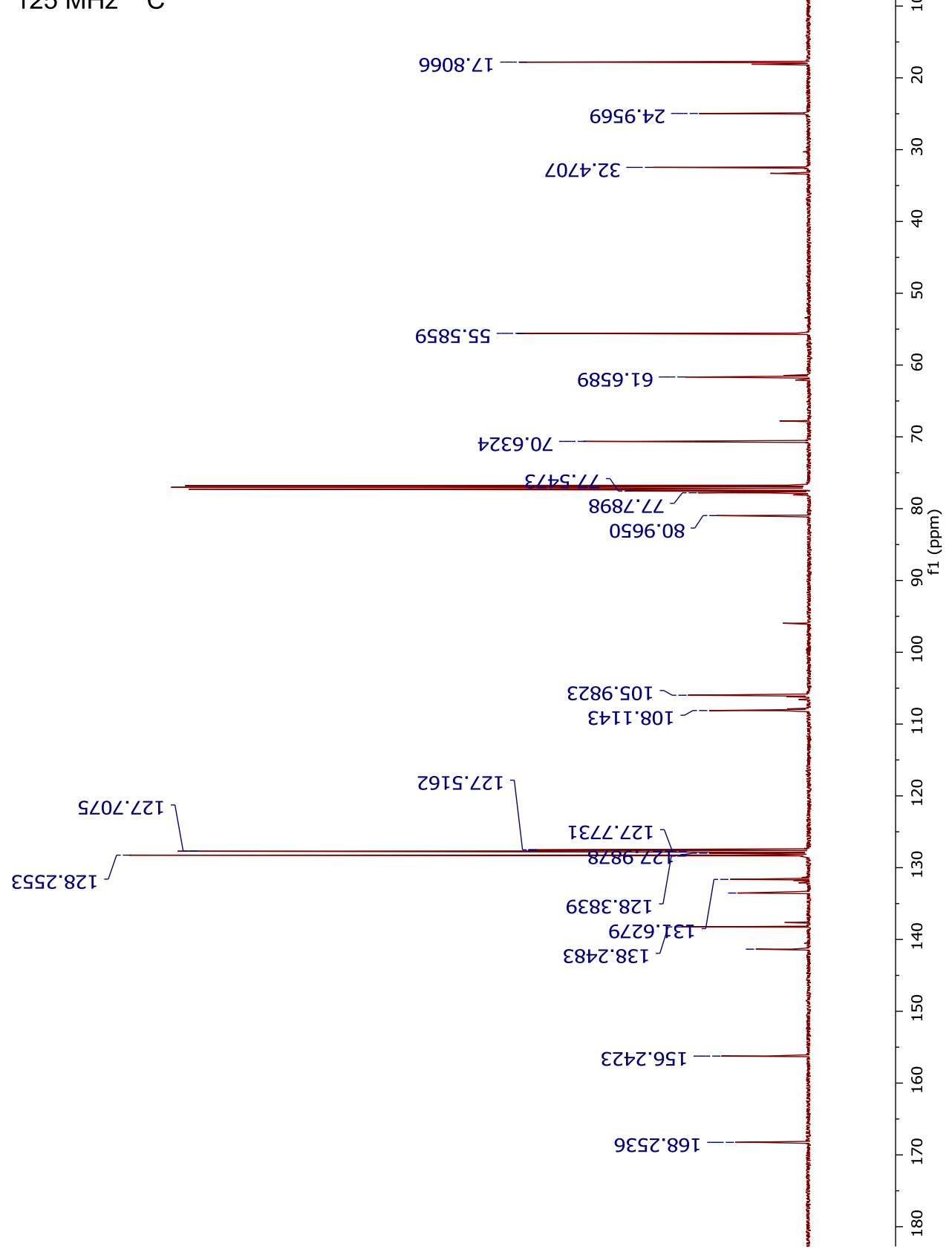




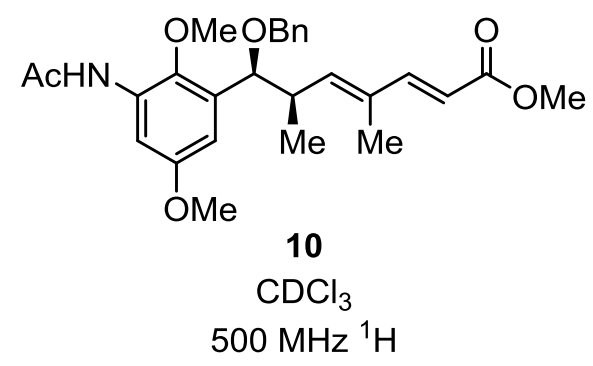

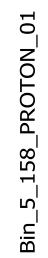

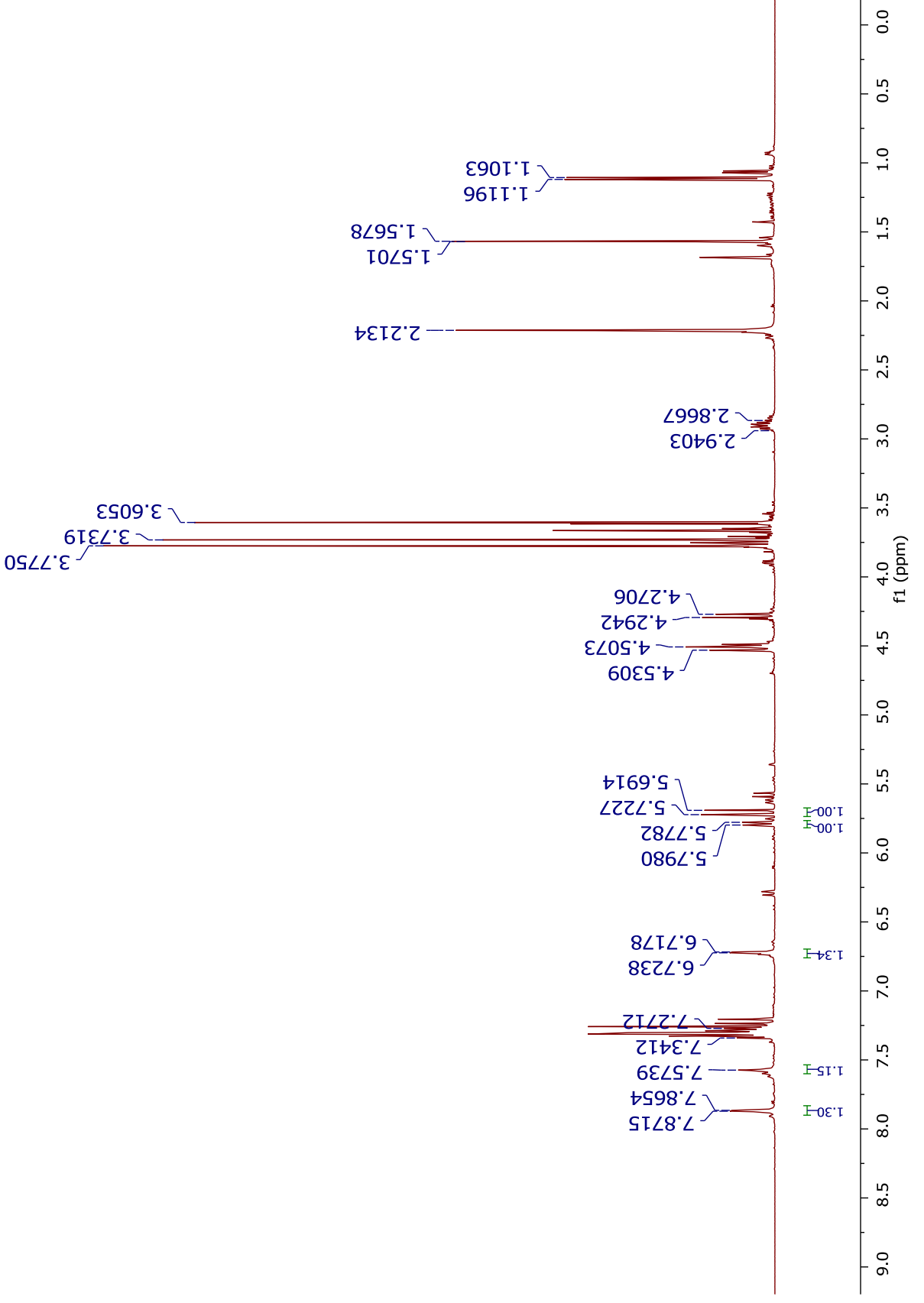



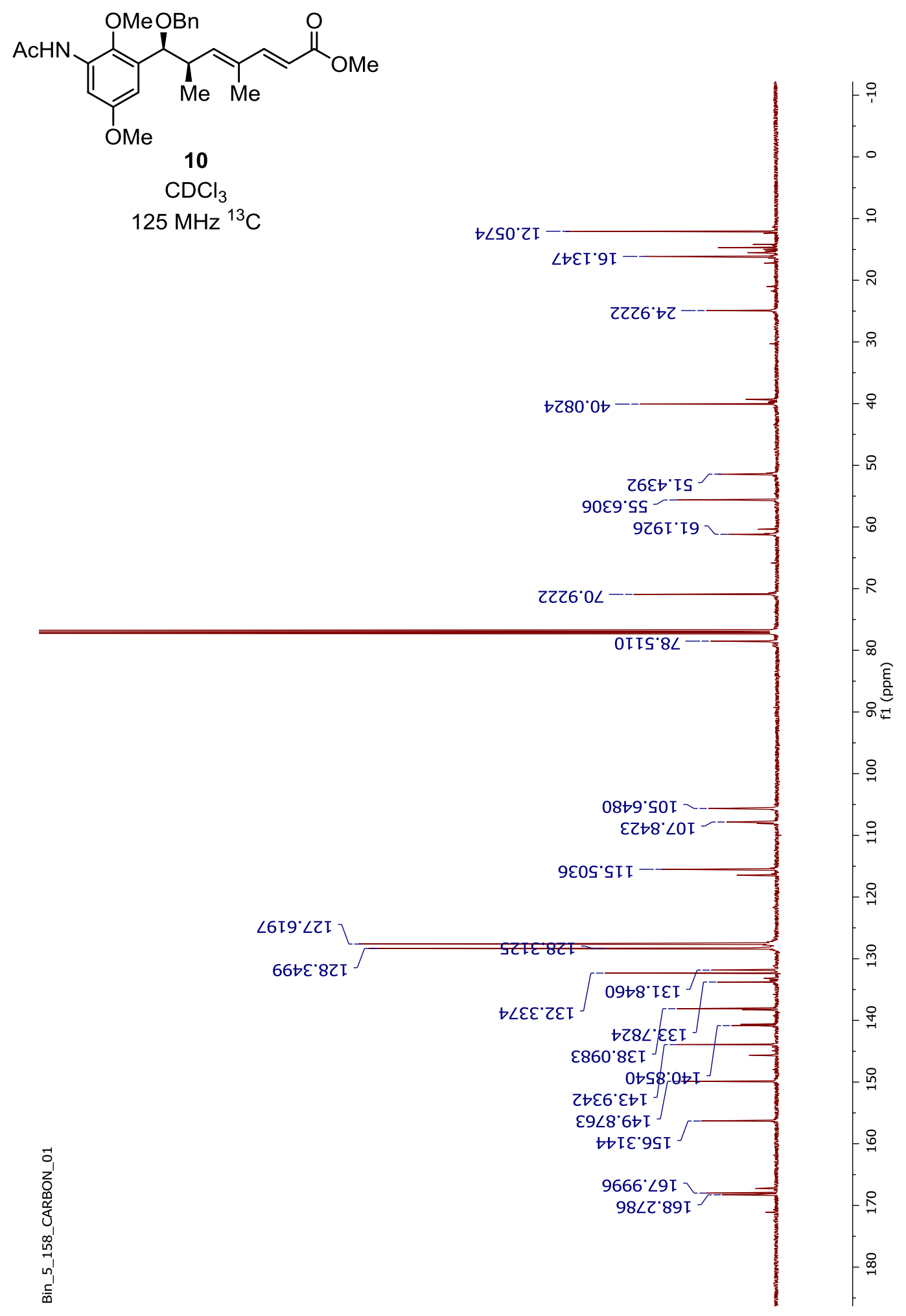


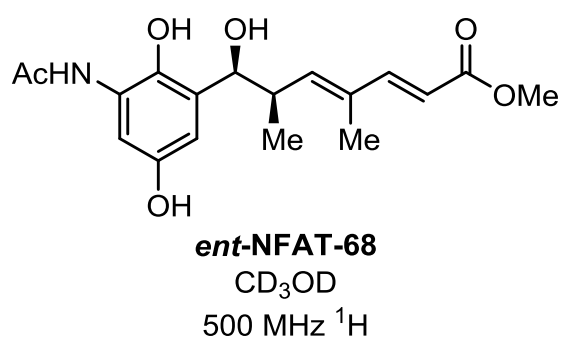

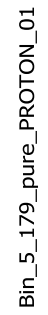

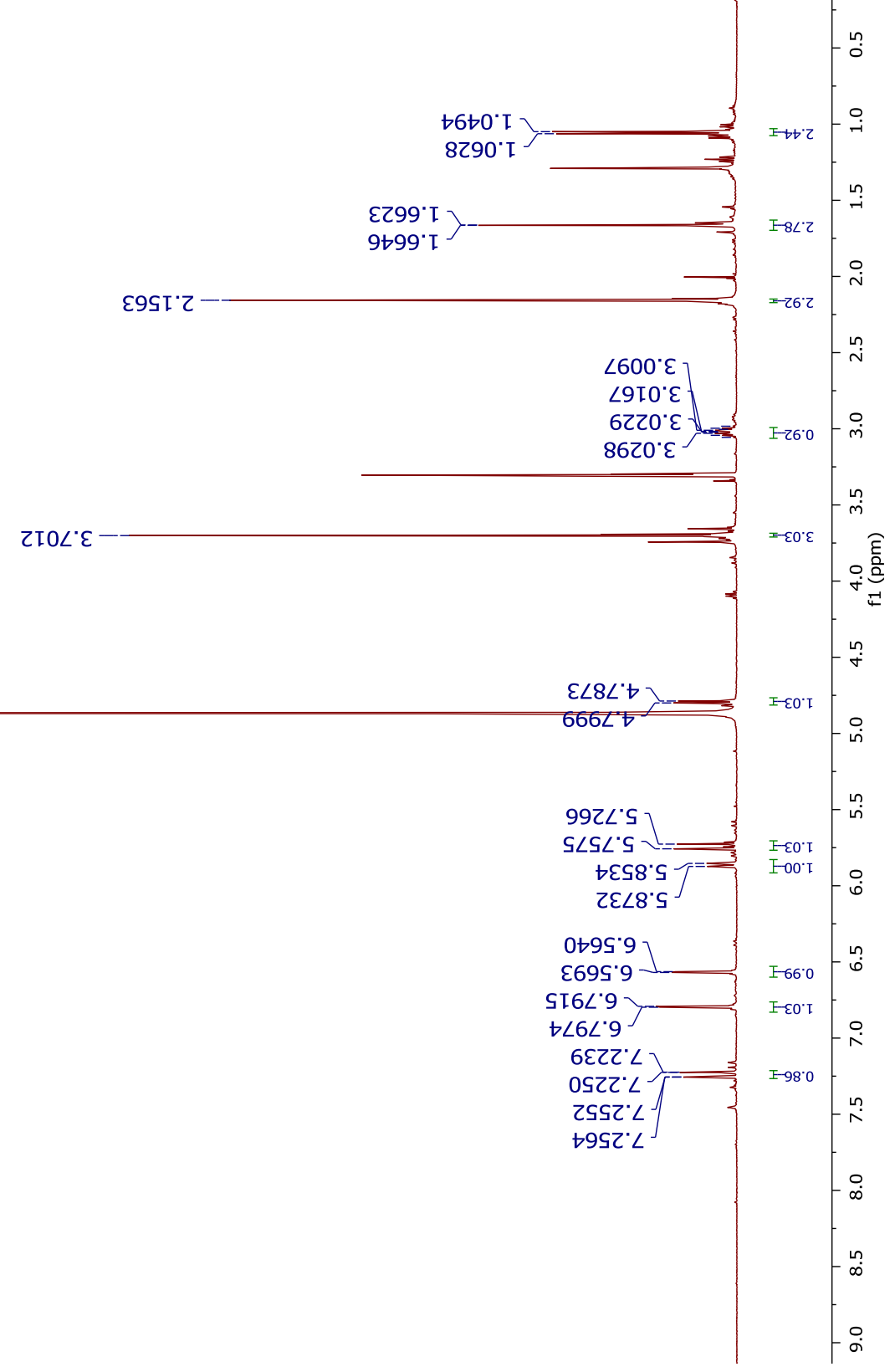




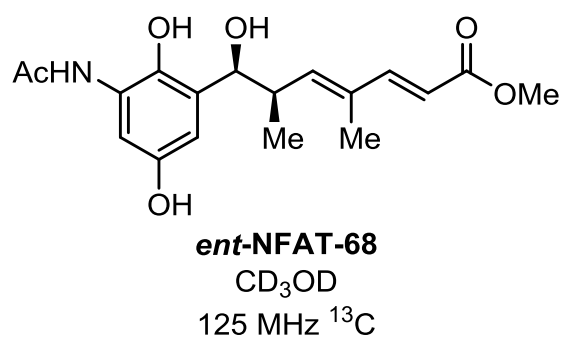

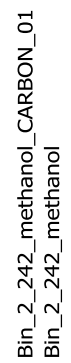

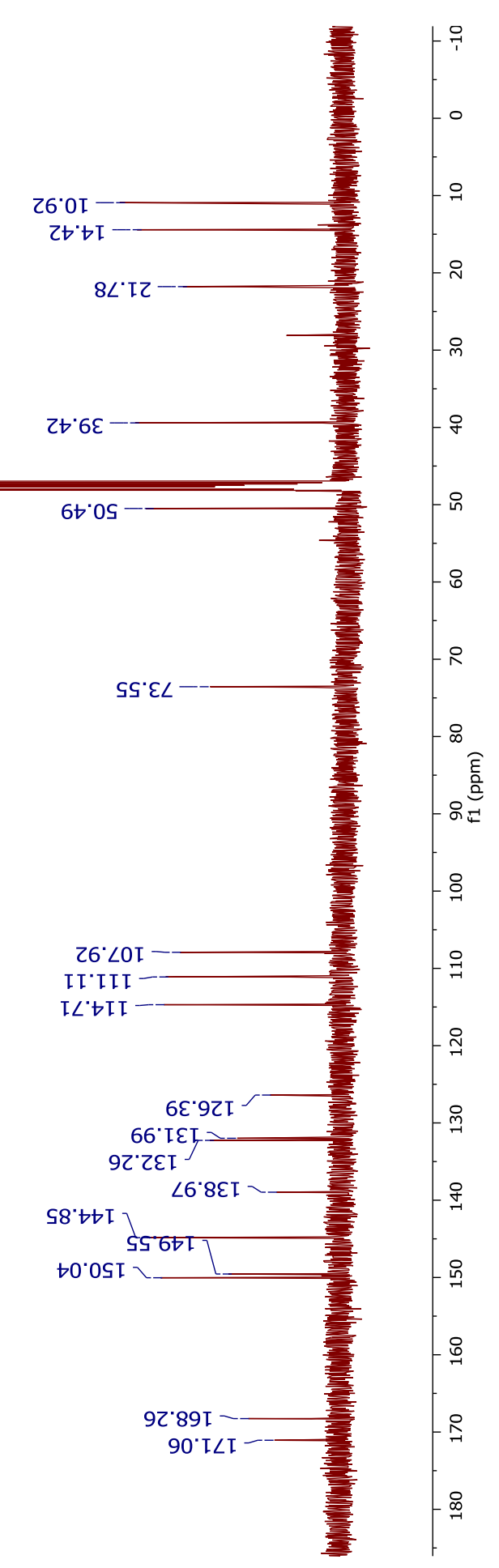




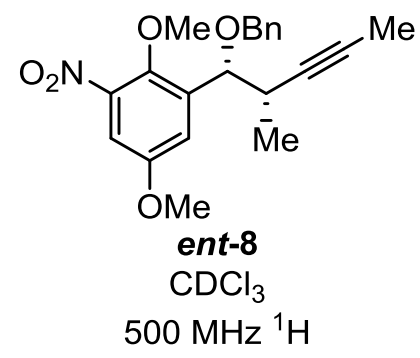

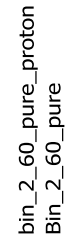

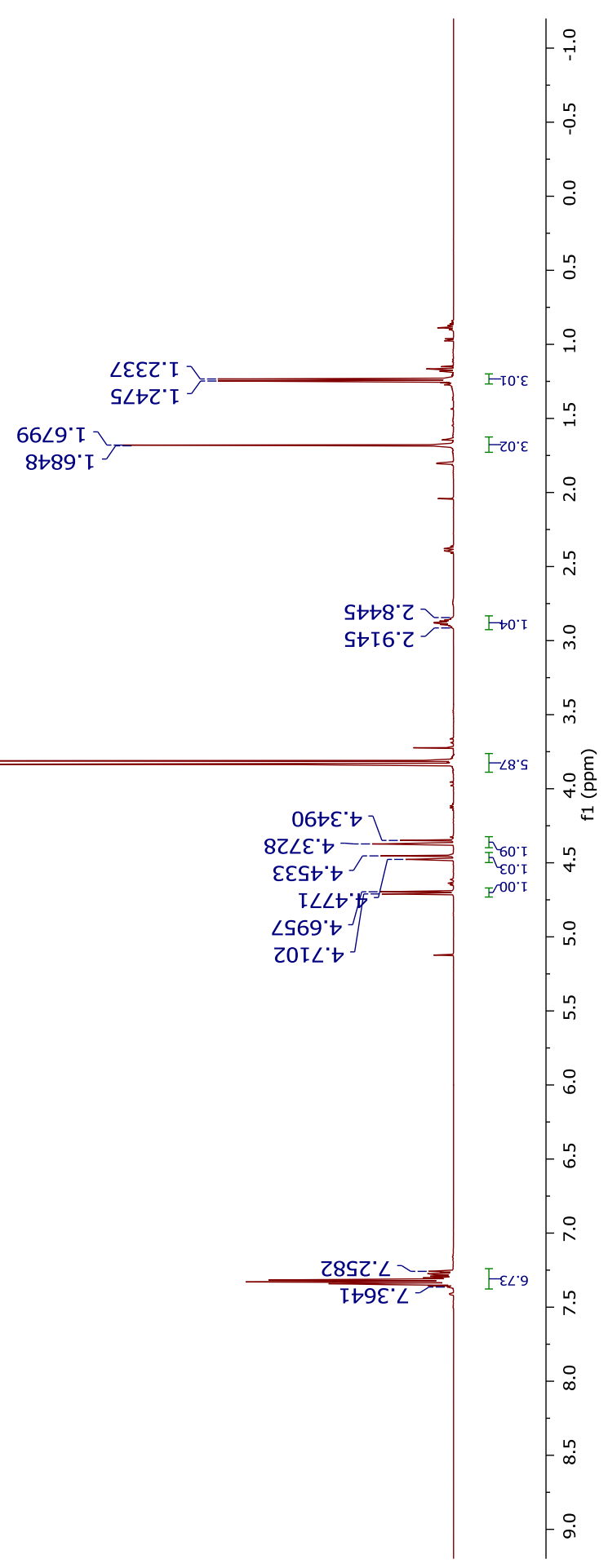



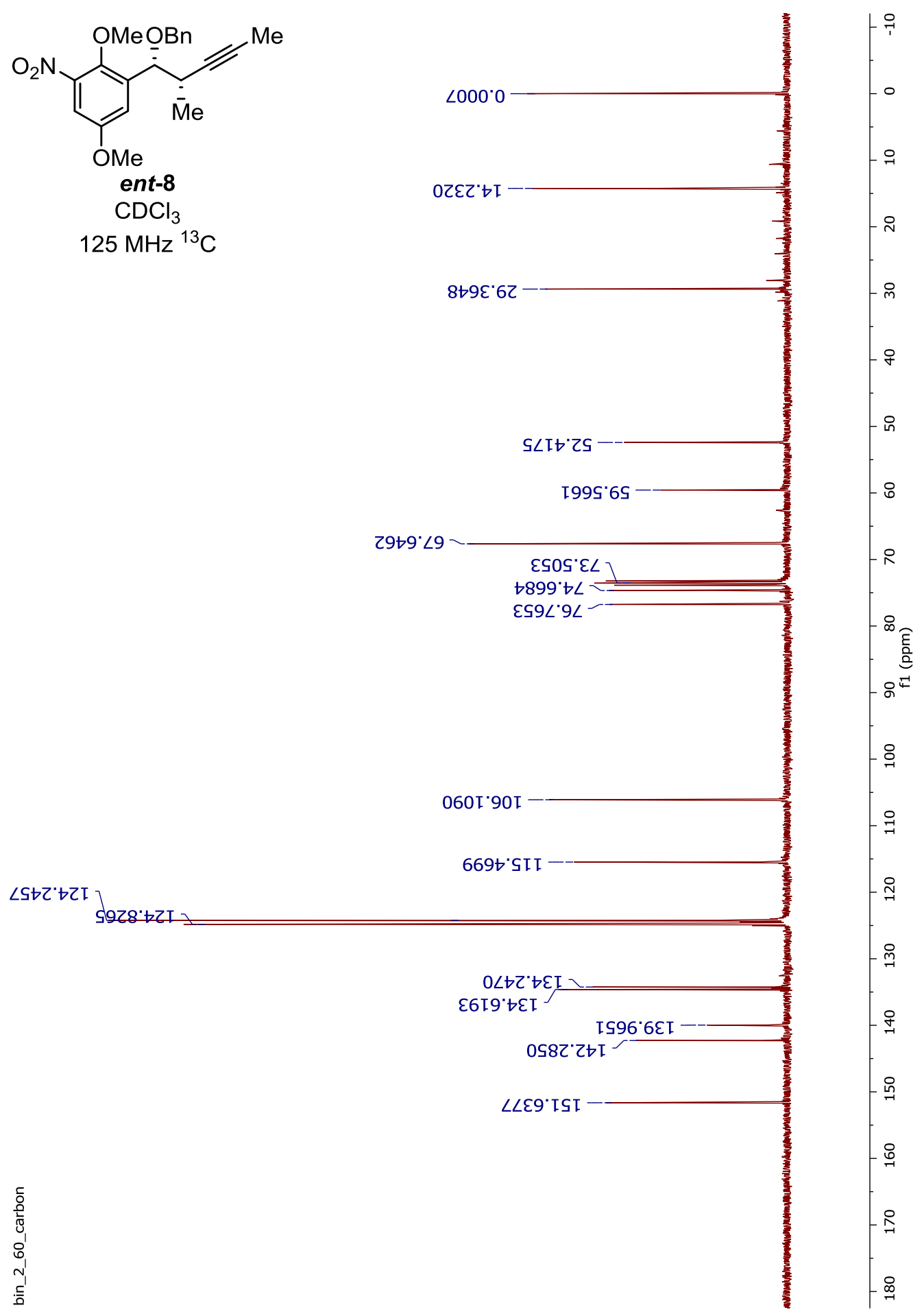


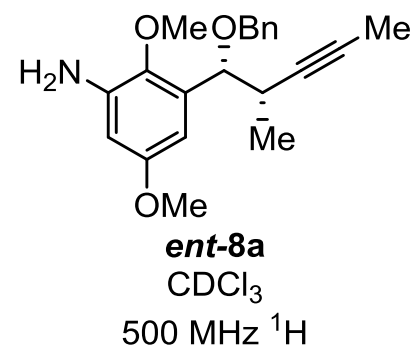

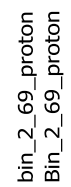

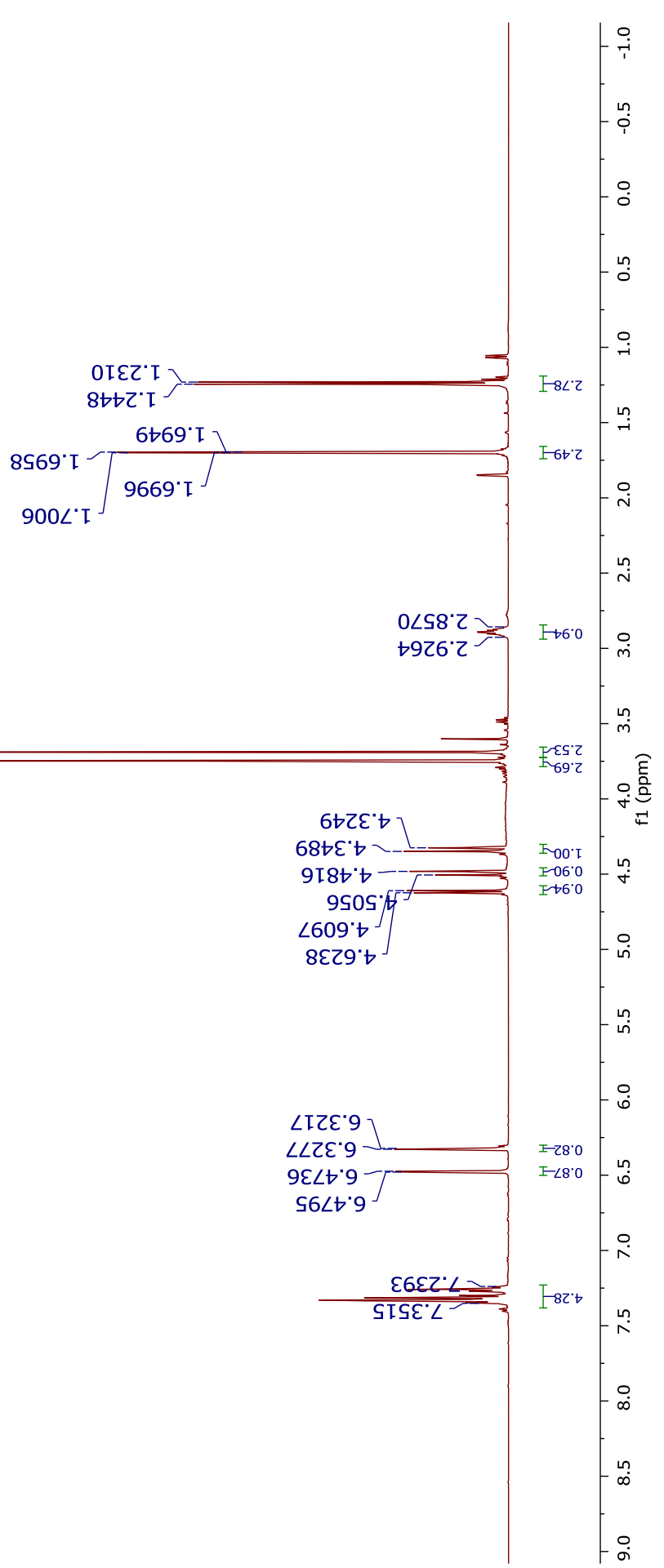



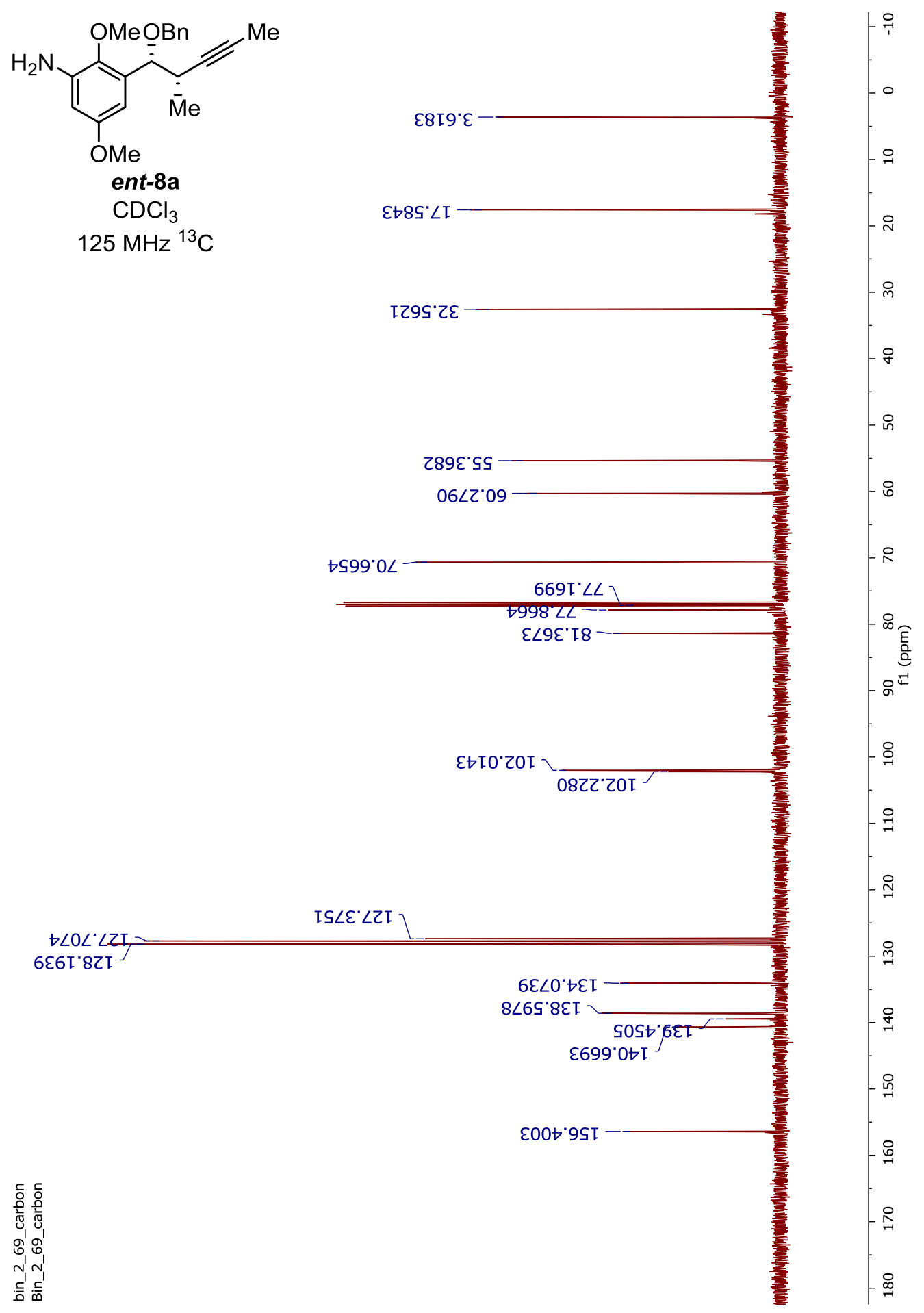


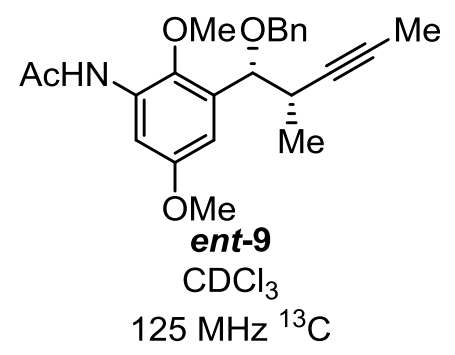

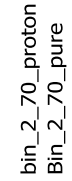

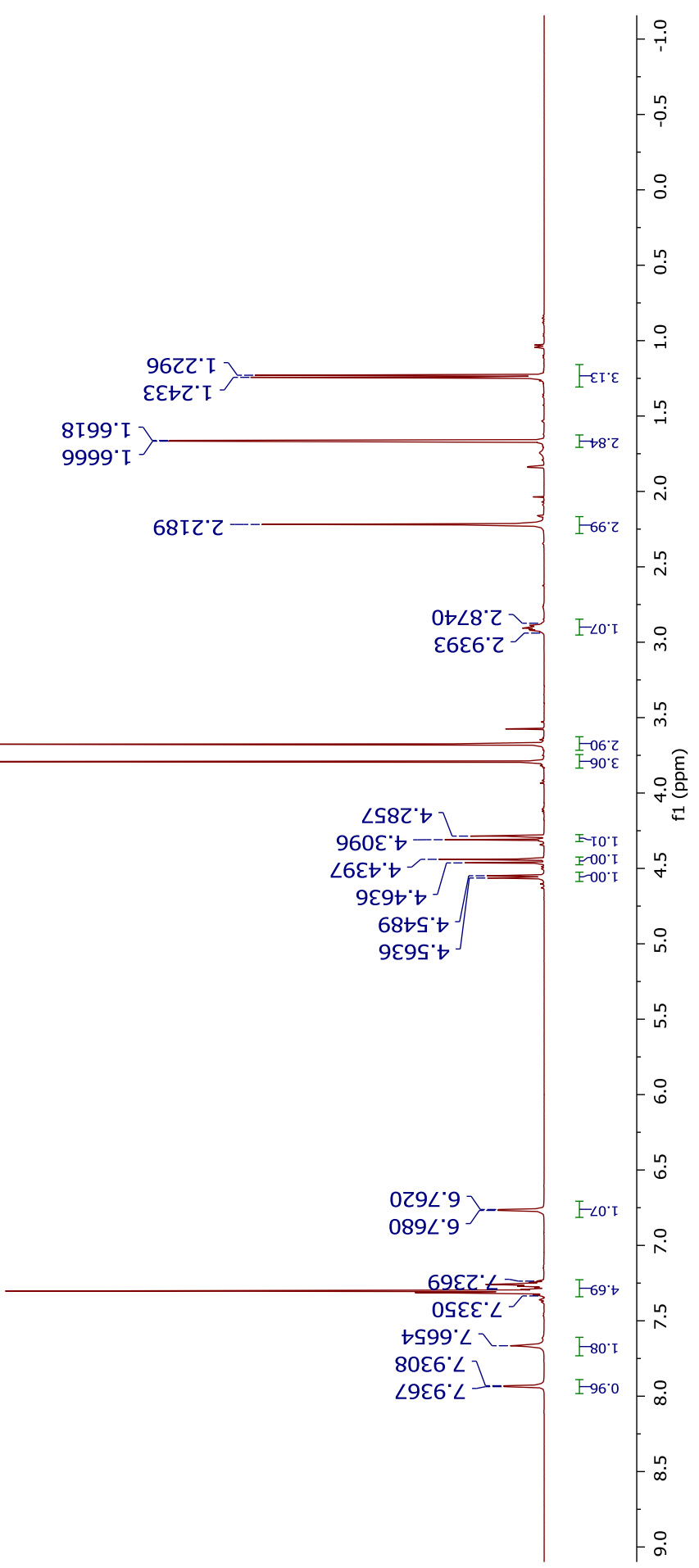



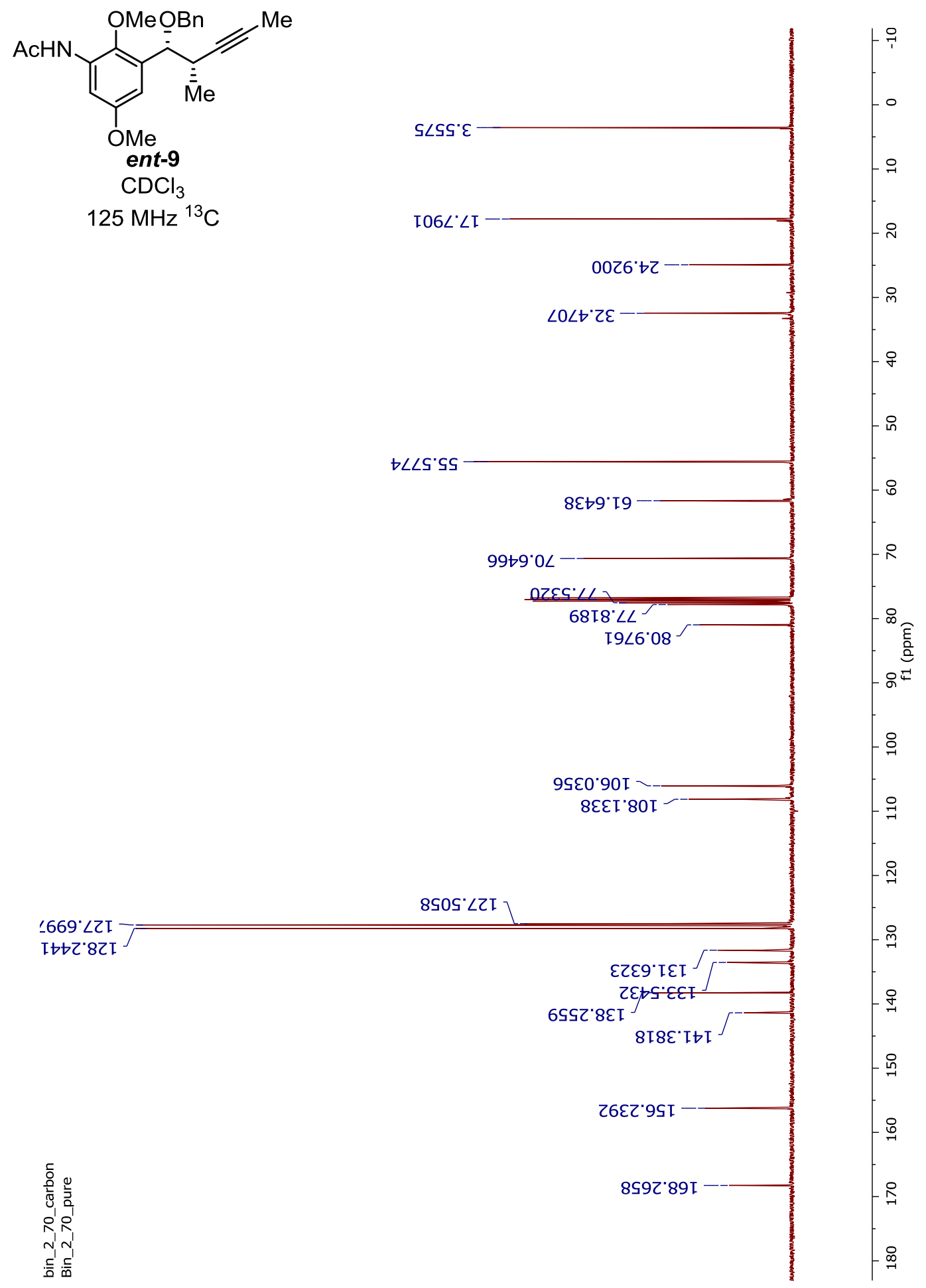

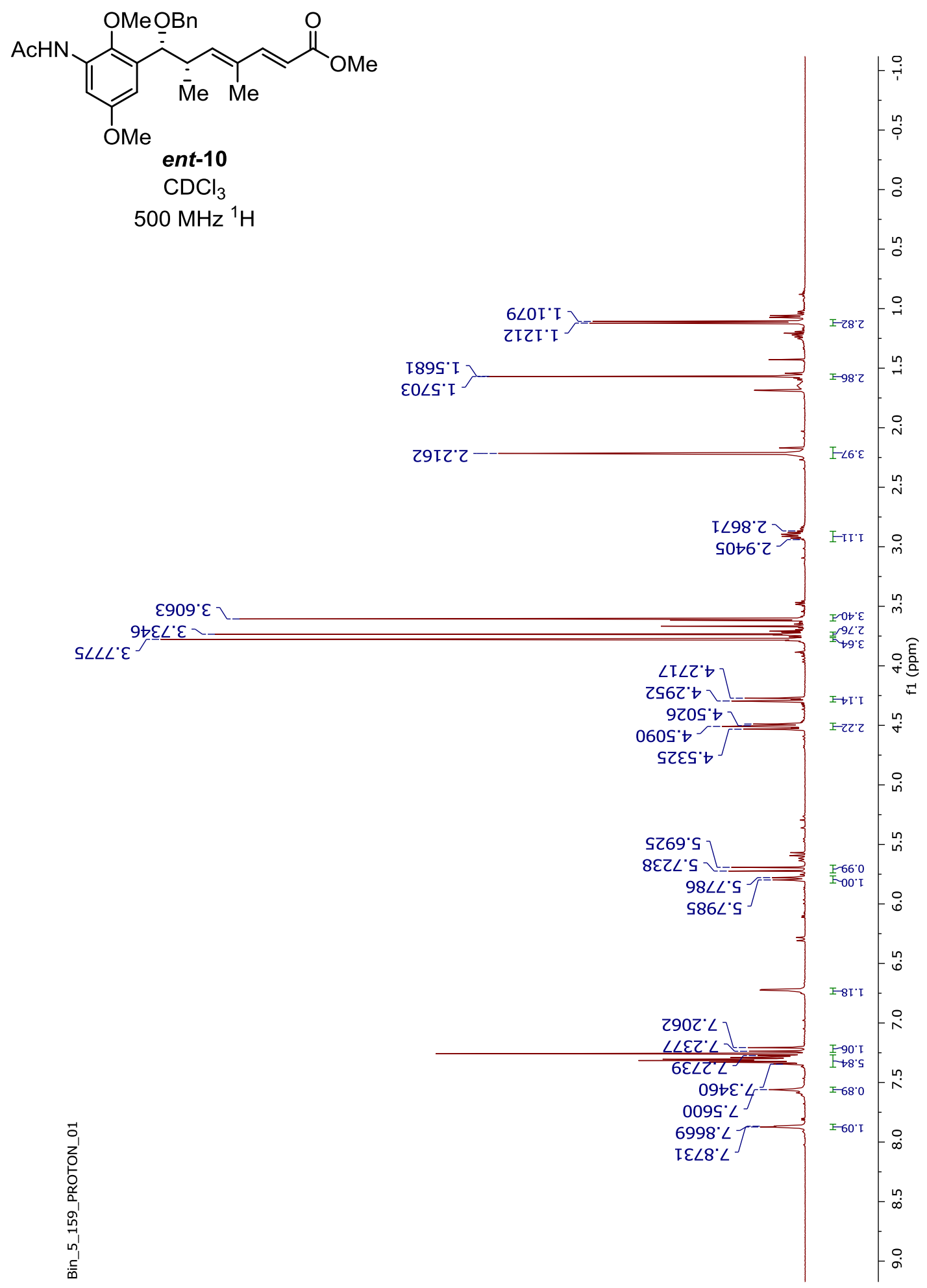

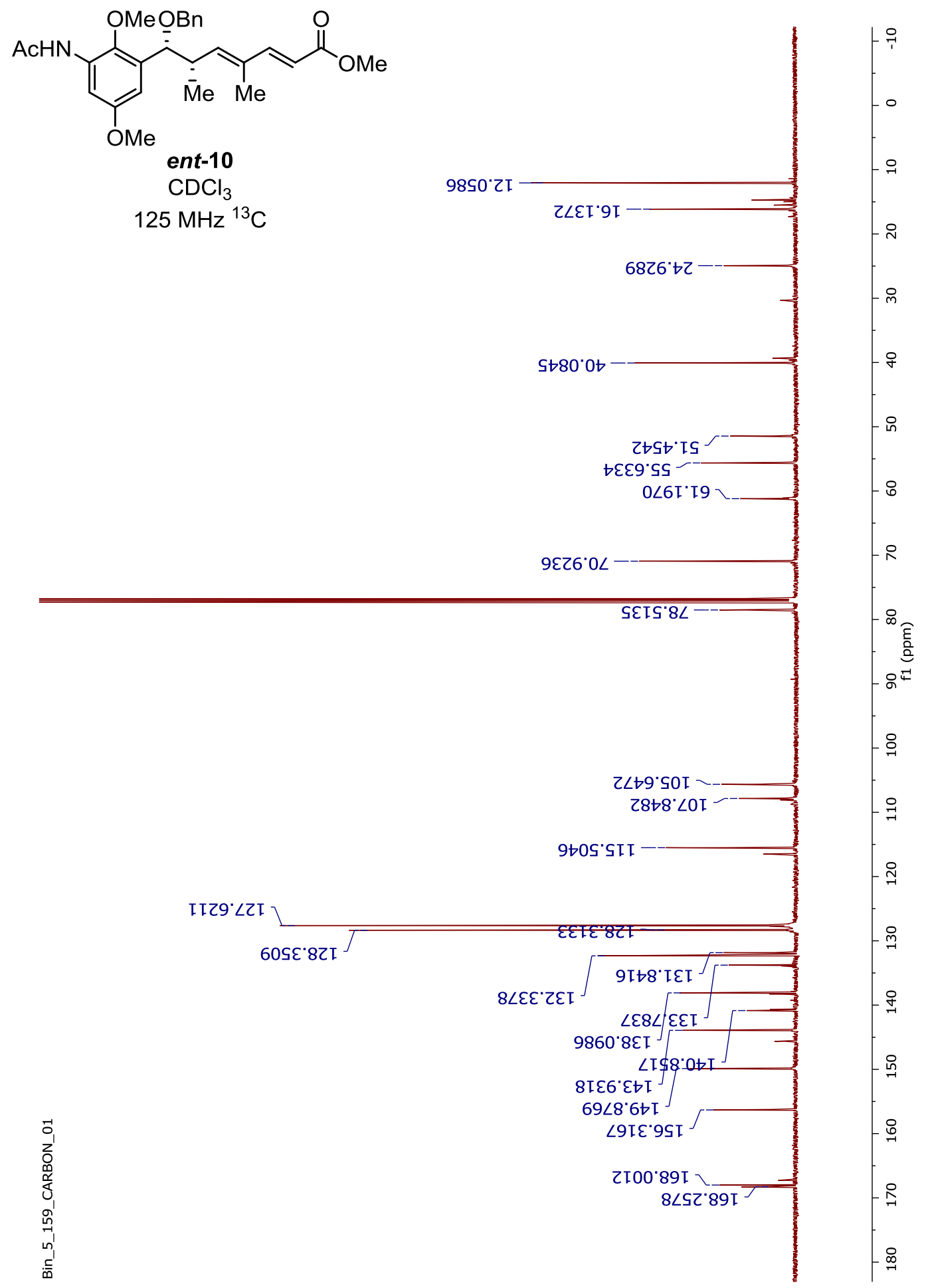

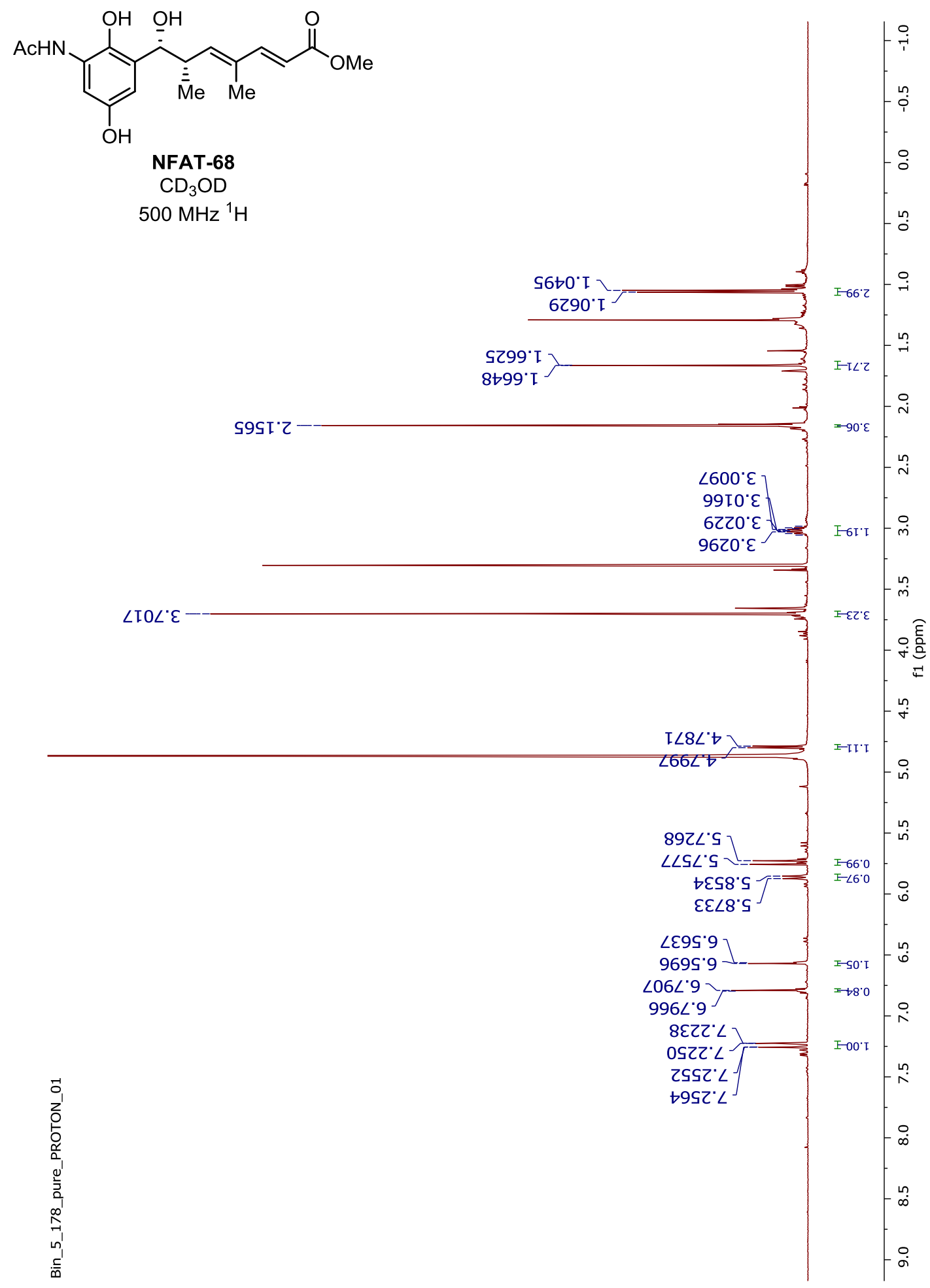

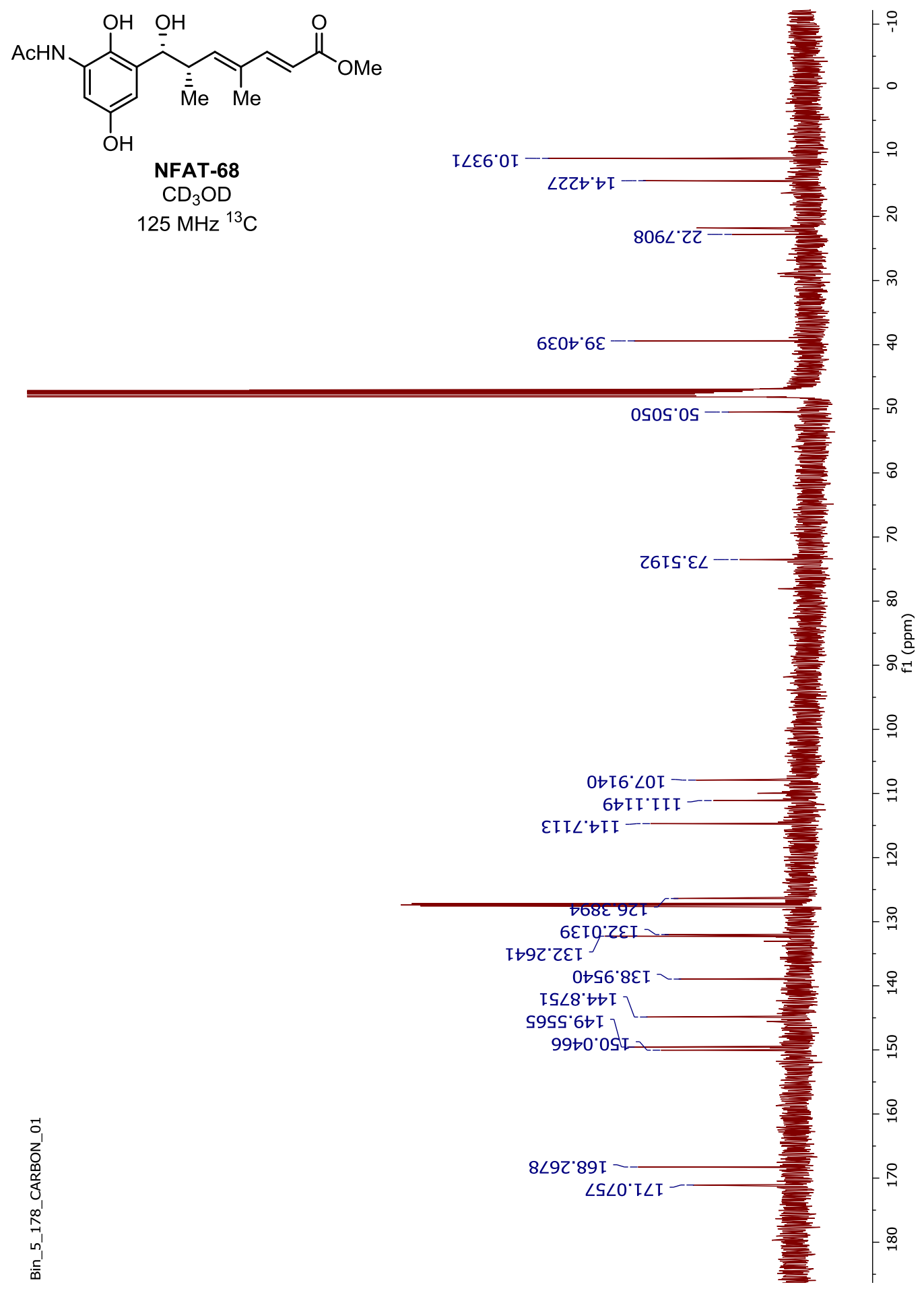


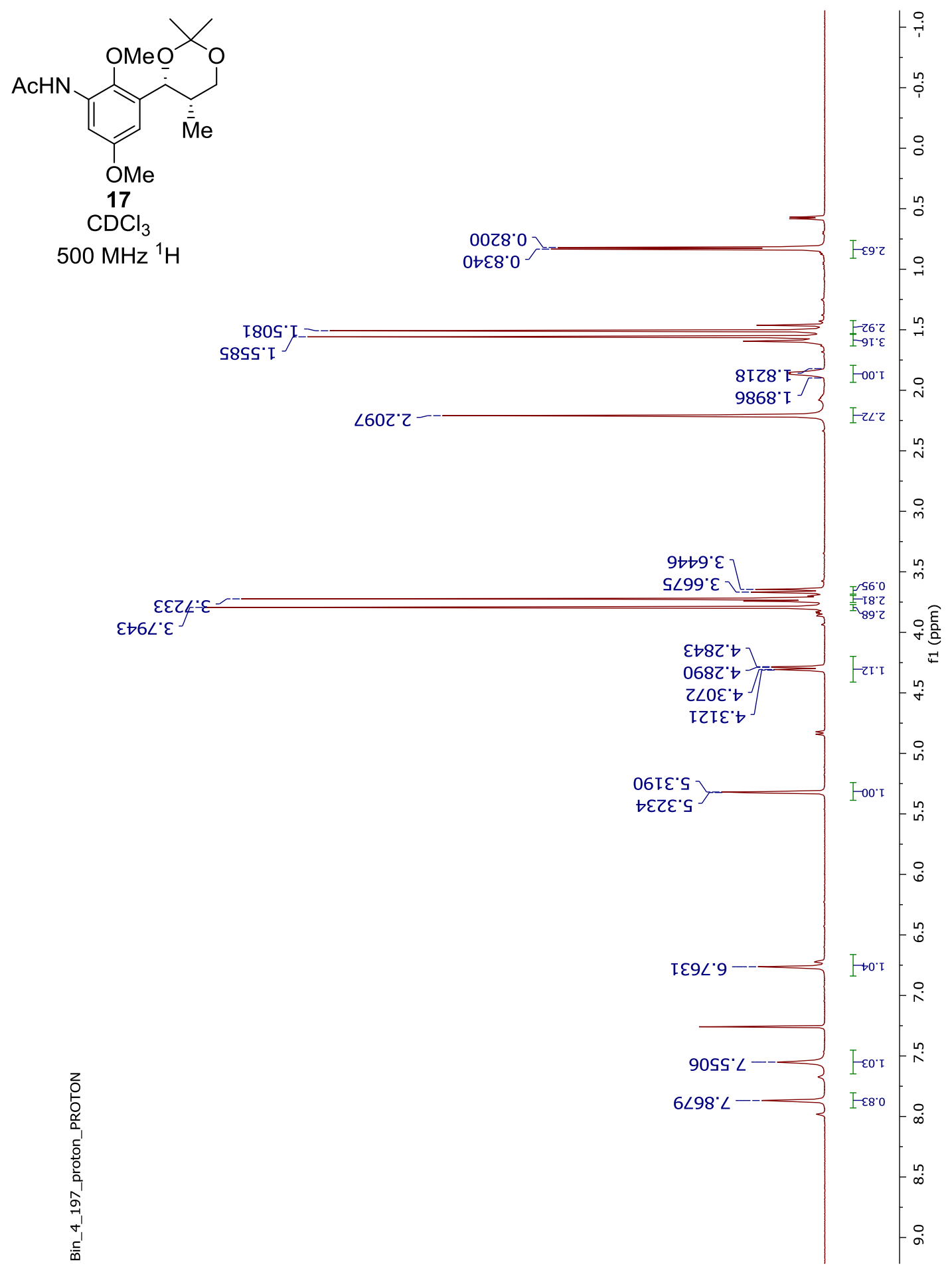




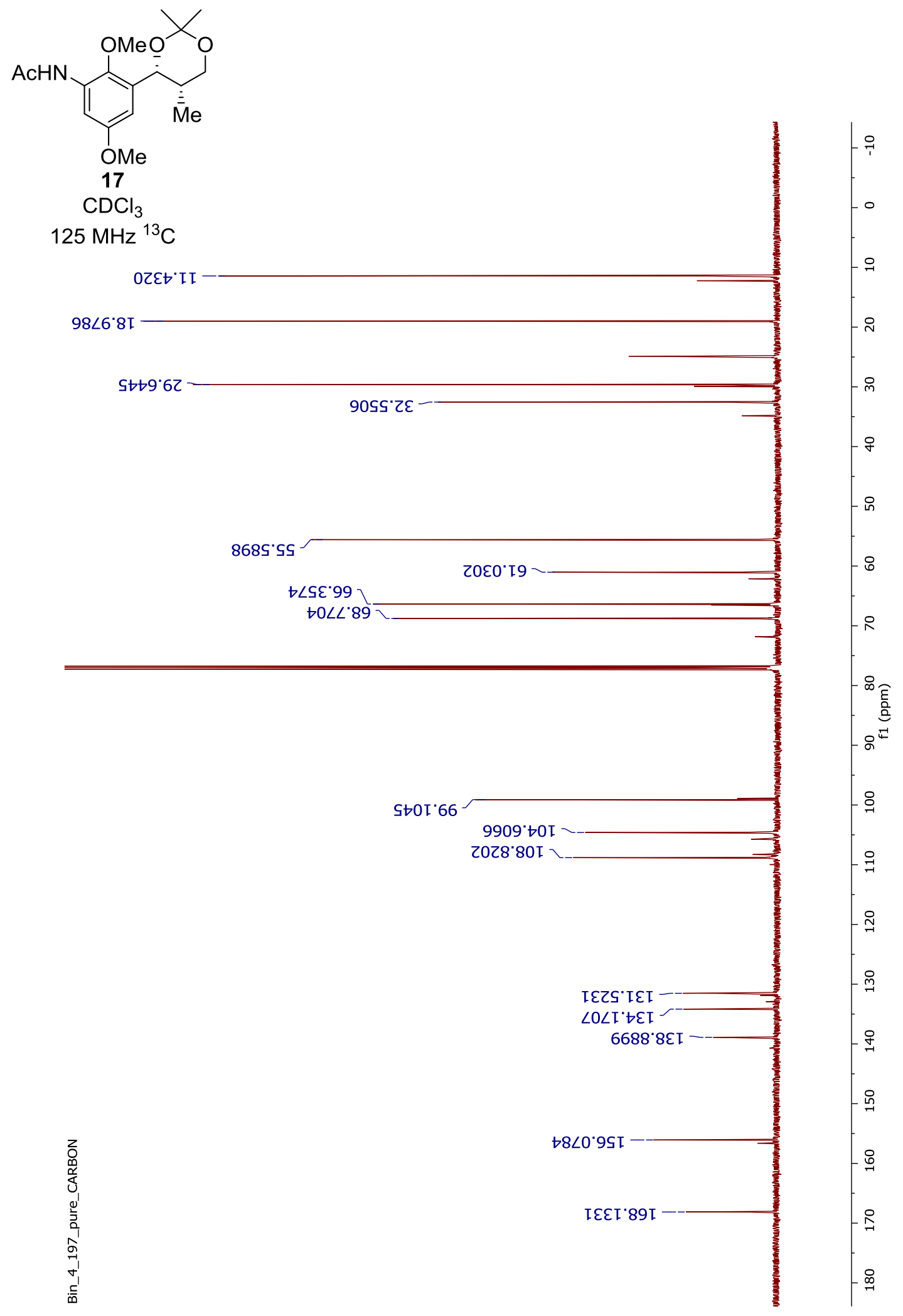




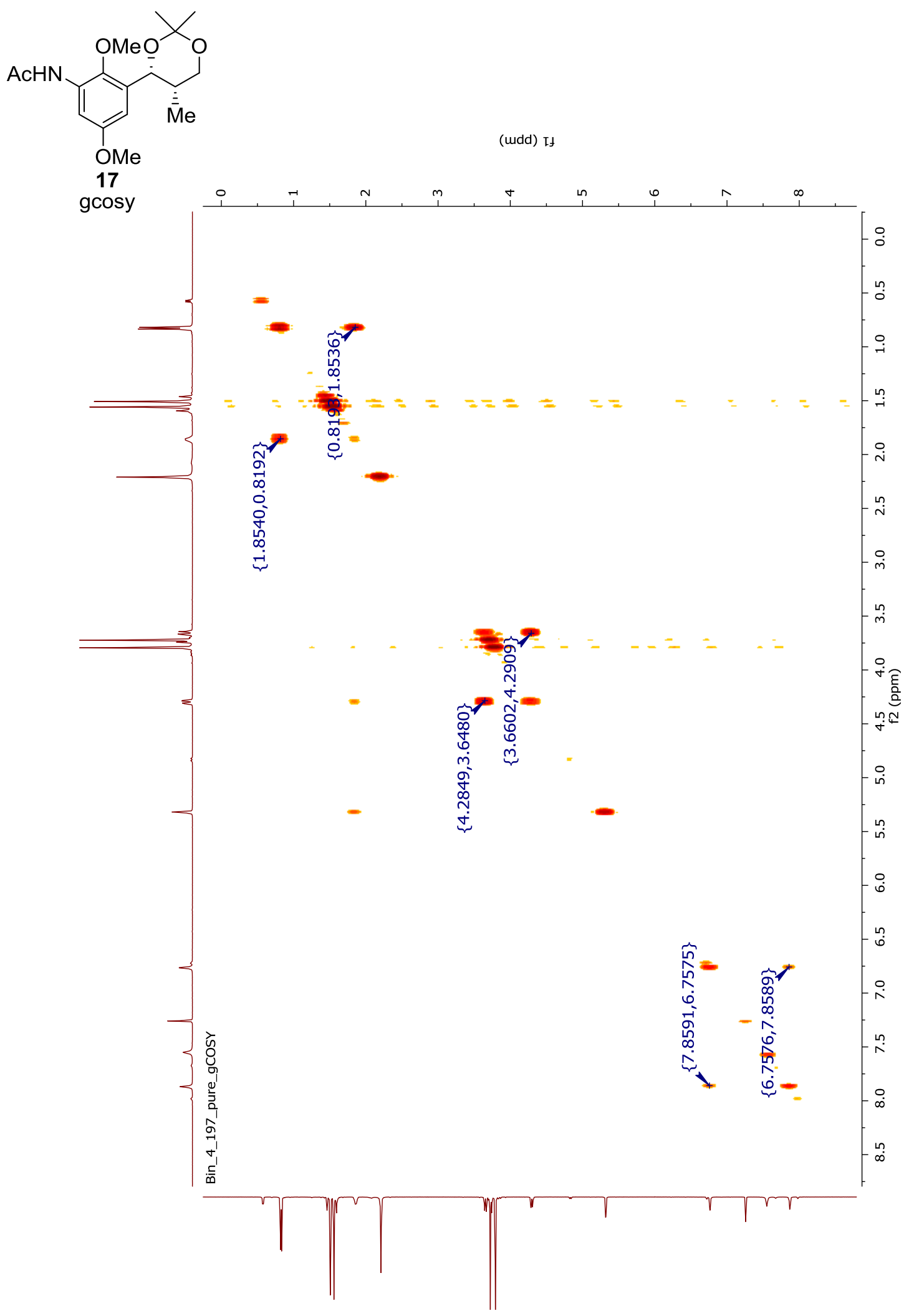

SI-100 


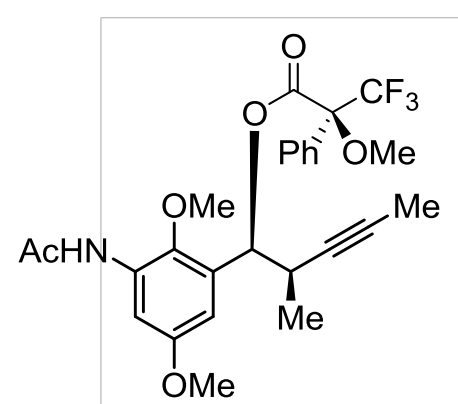

(S)-19

$\mathrm{CDCl}_{3}$ $500 \mathrm{MHz}^{1} \mathrm{H}$

$6 乙 L ' \varepsilon$ ${ }^{\circ} 8^{\circ} \varepsilon$

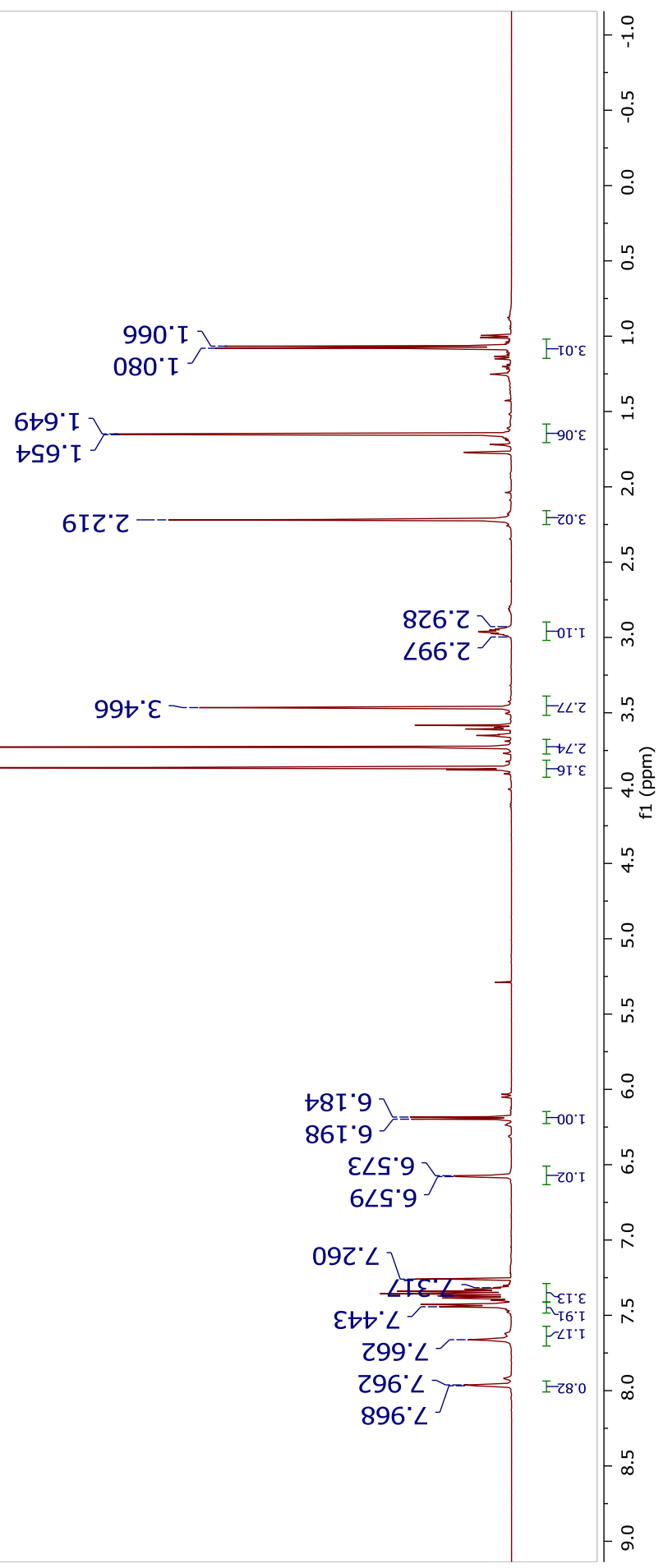

SI-101 

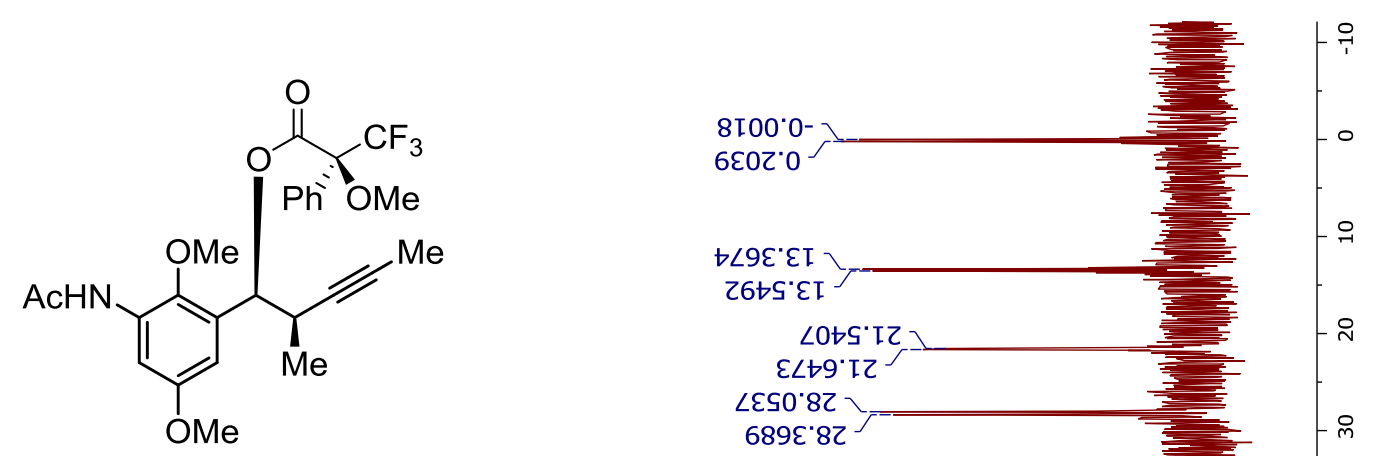

(S)-19

$\mathrm{CDCl}_{3}$

$100 \mathrm{MHz}{ }^{13} \mathrm{C}$

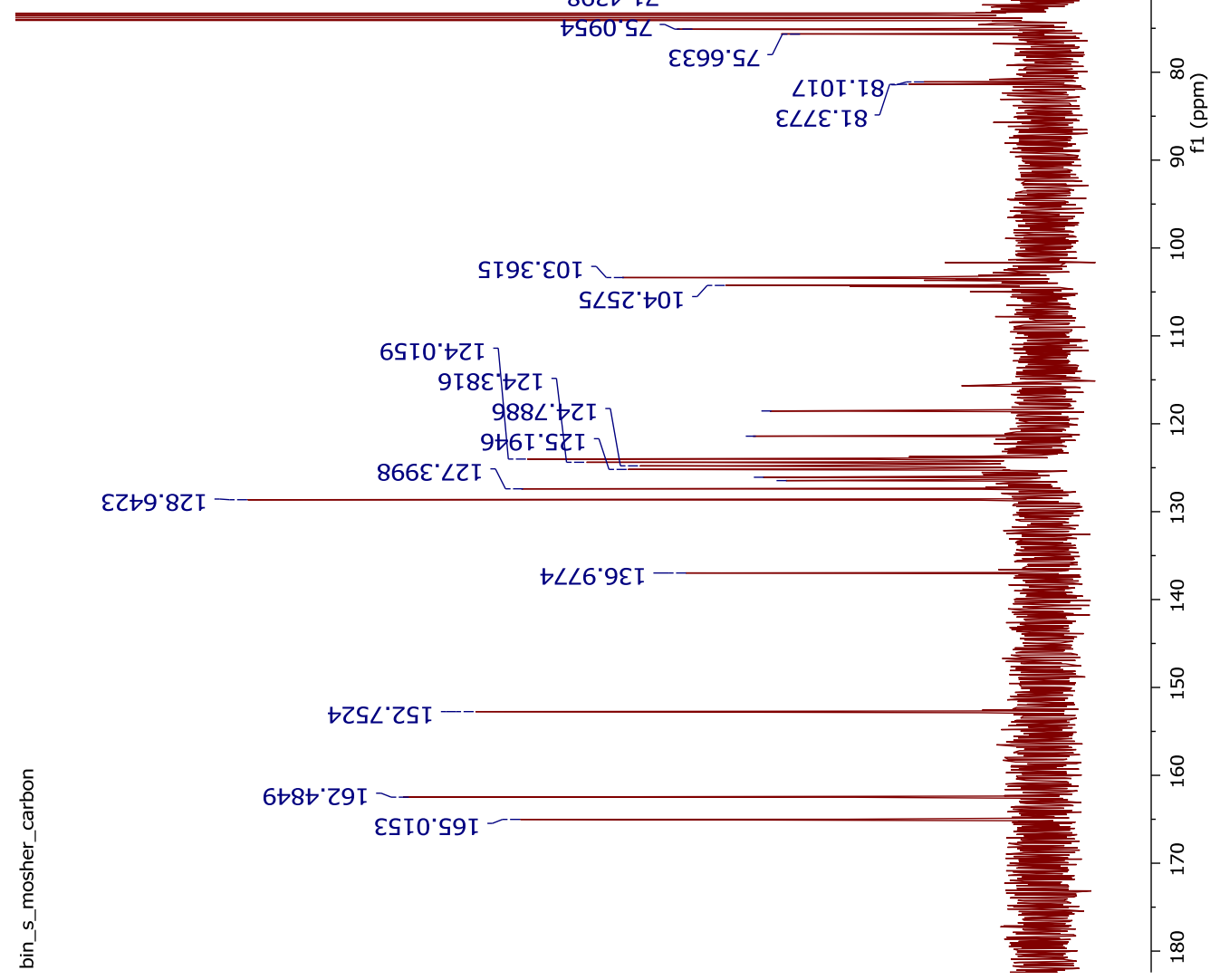

SI-102 

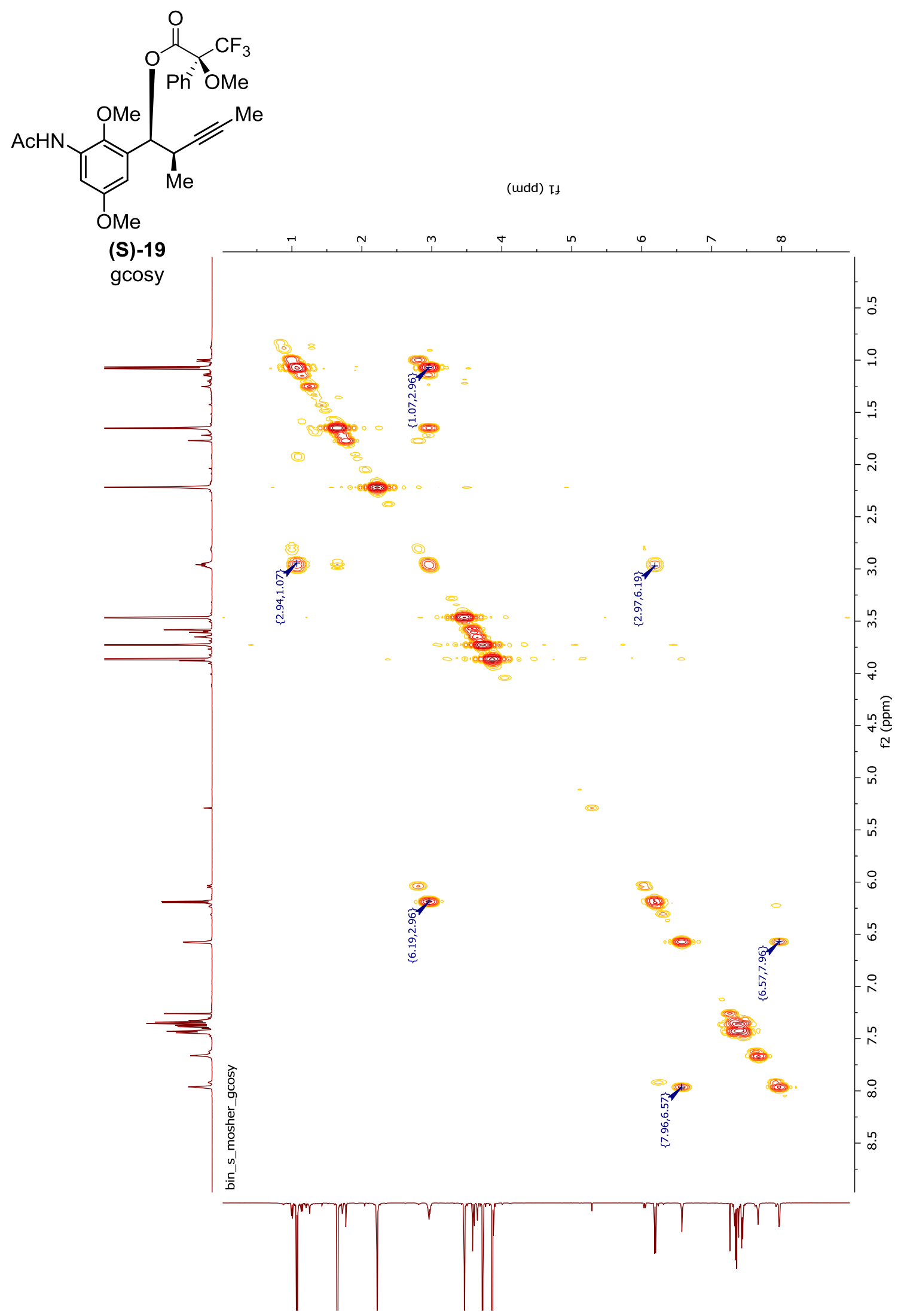

SI-103 

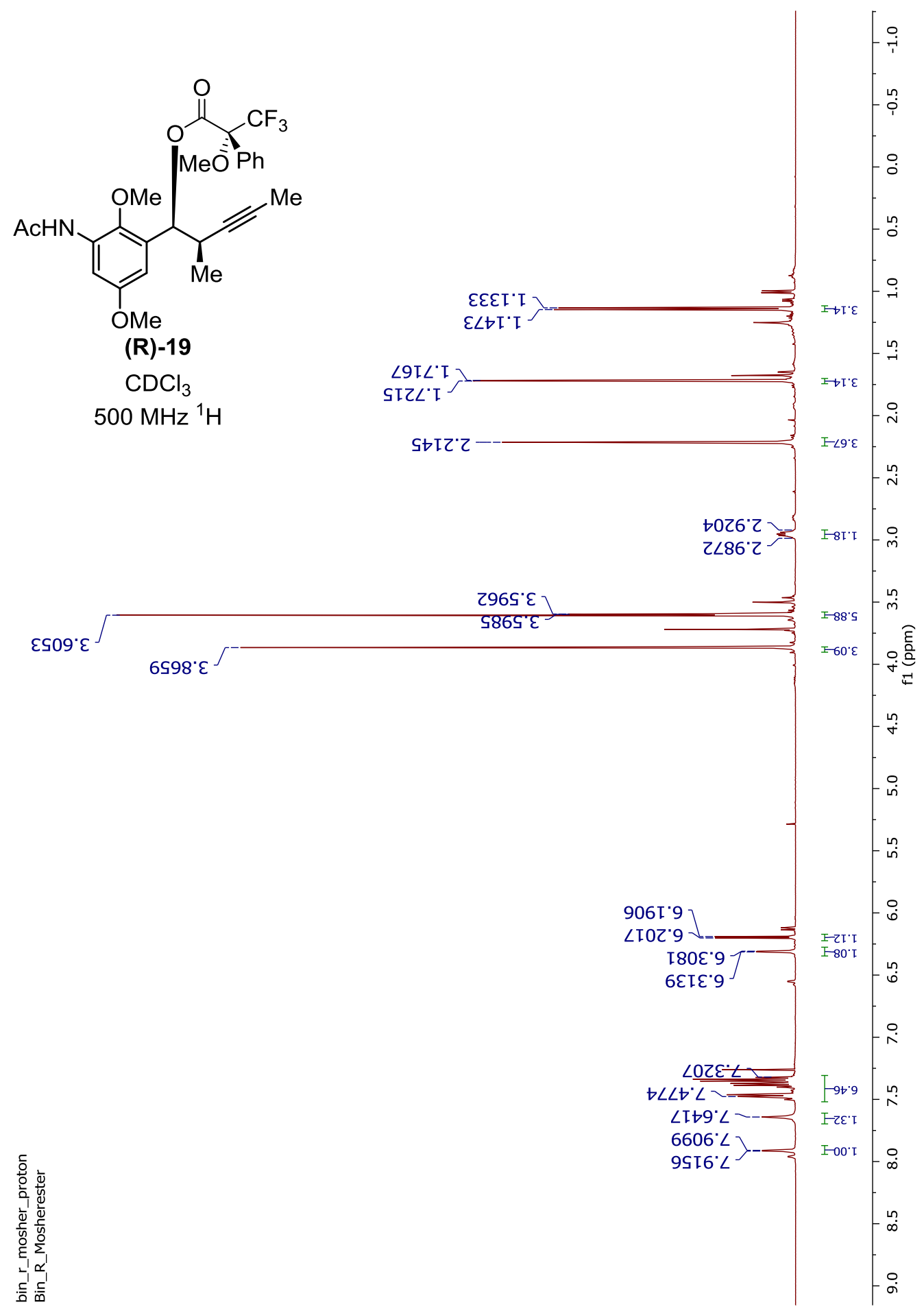

SI-104 


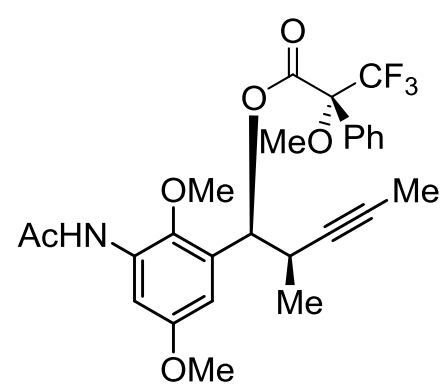

(R)-19

$\mathrm{CDCl}_{3}$ $125 \mathrm{MHz}{ }^{13} \mathrm{C}$

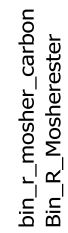

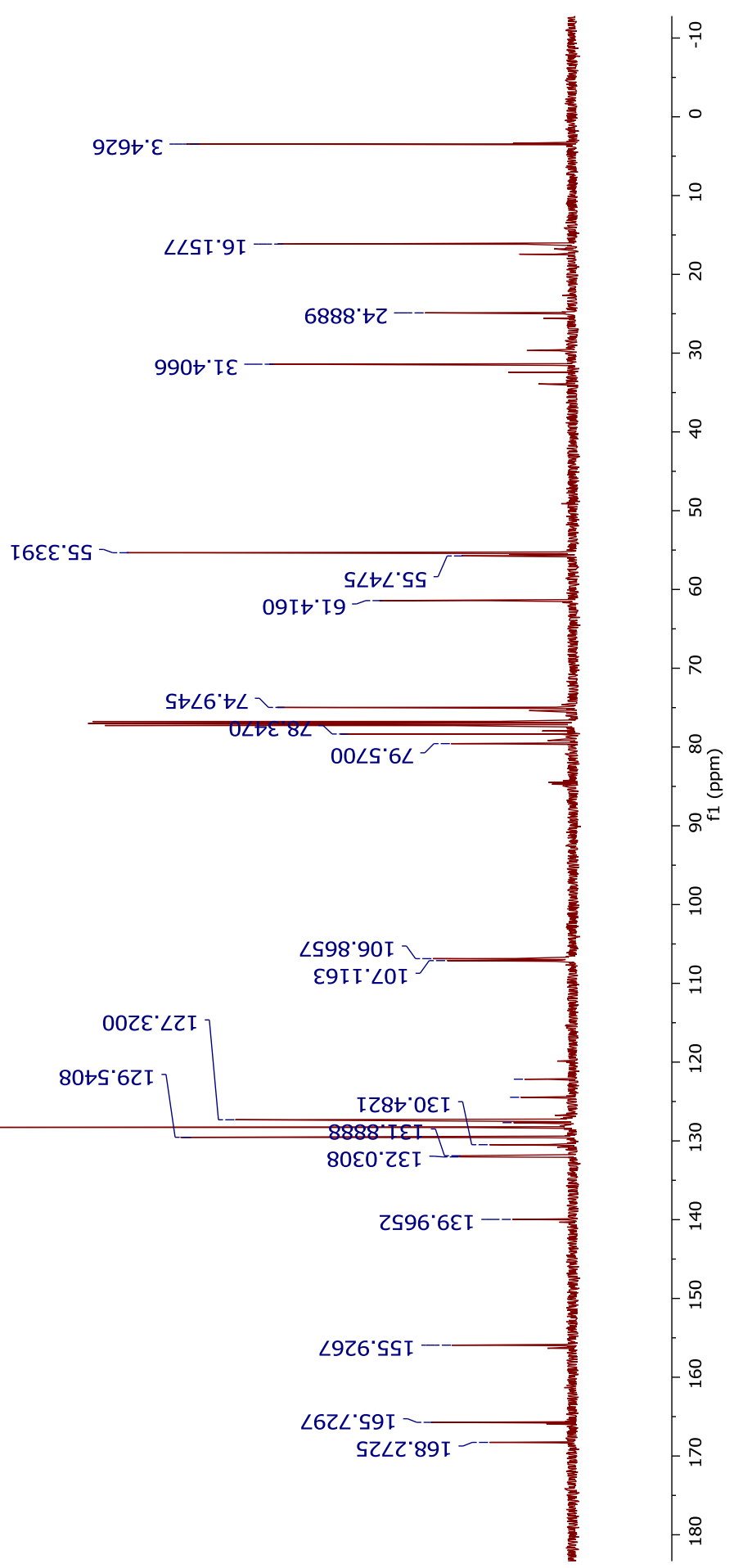

SI-105 


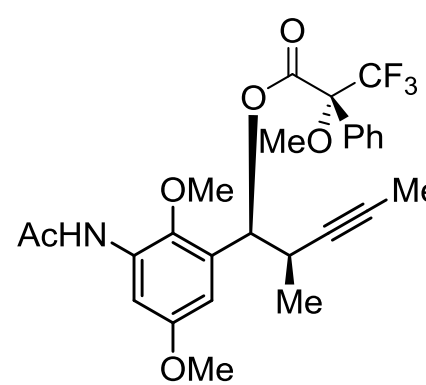

(R)-19 gcosy

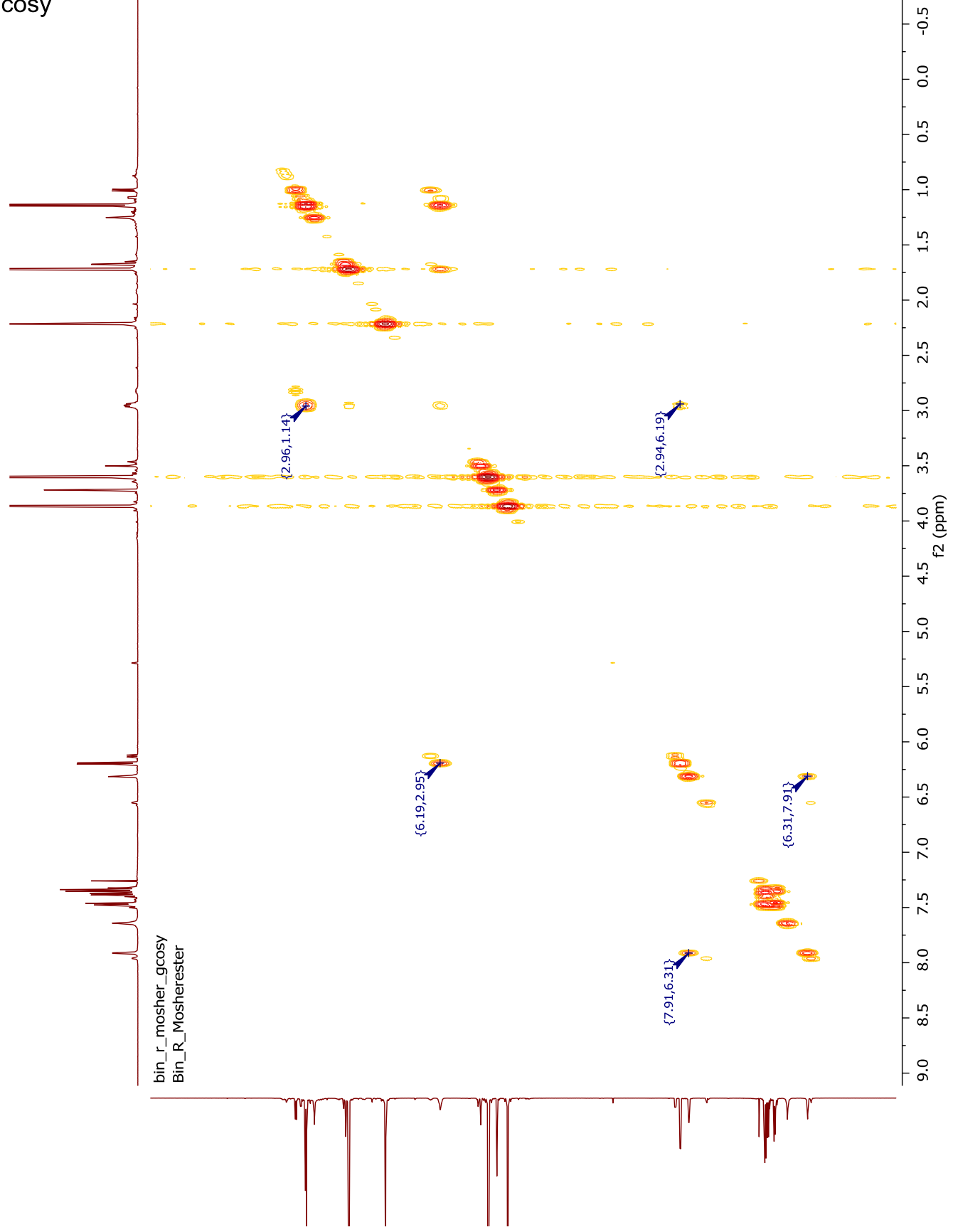




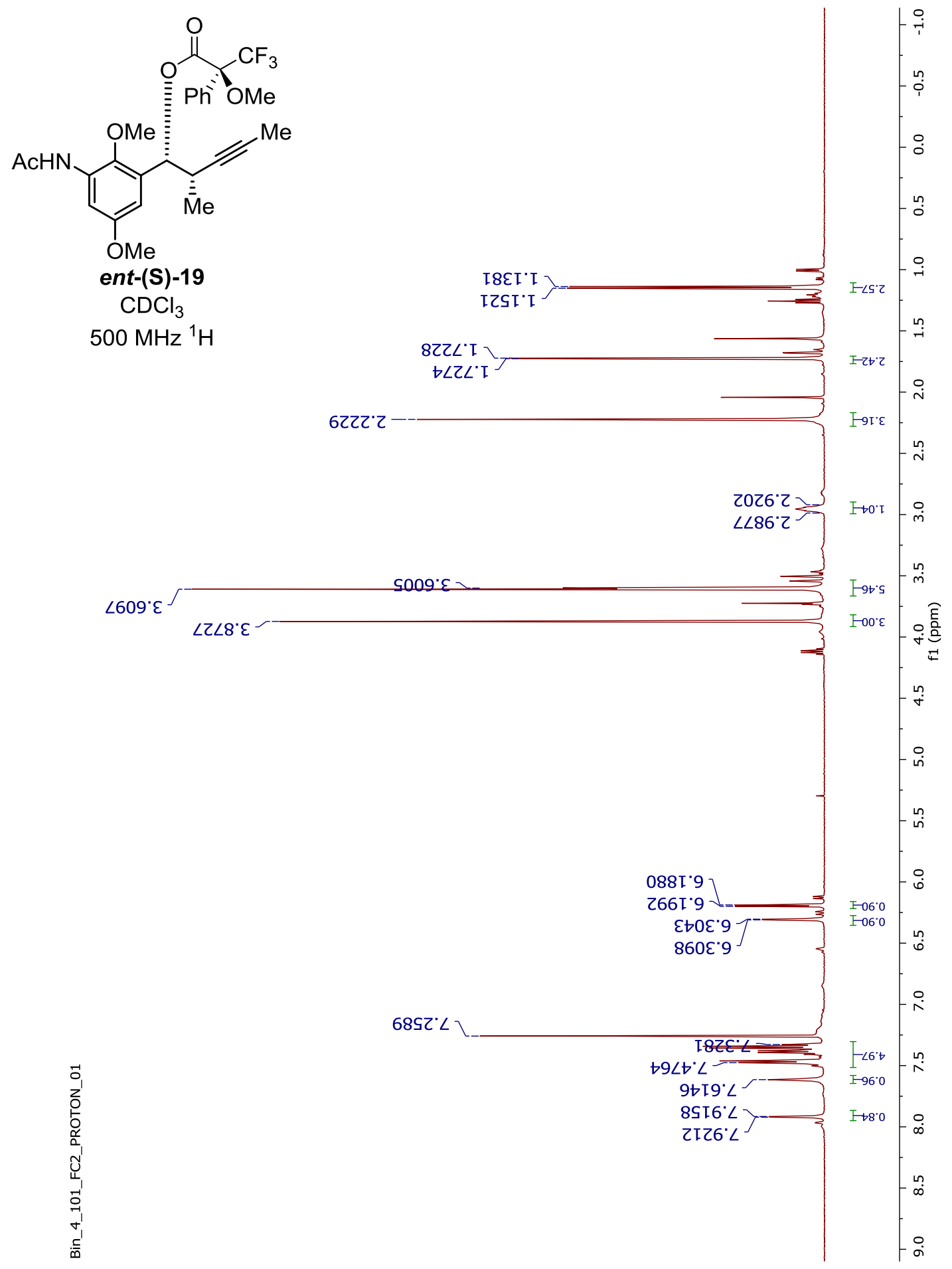



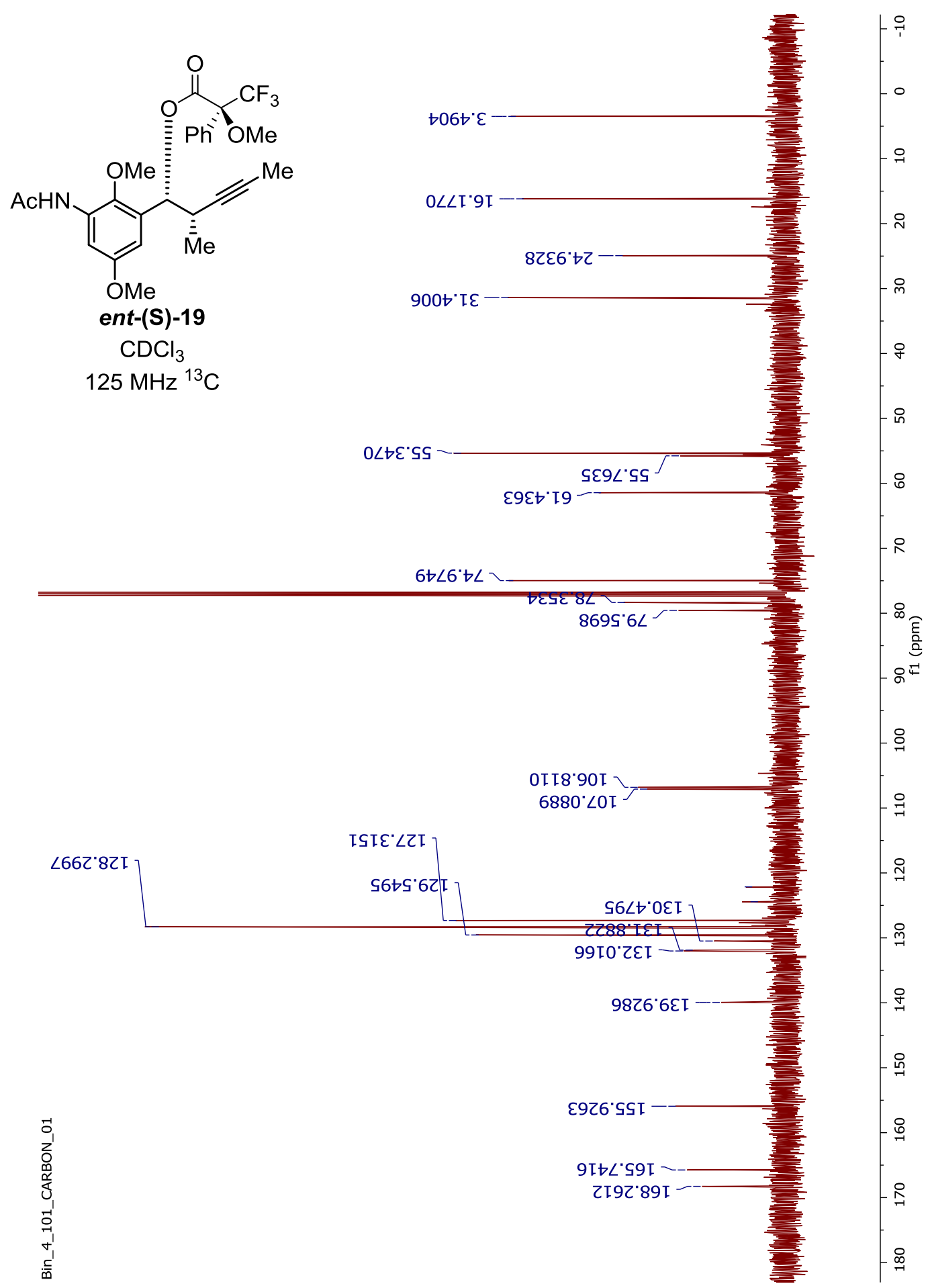

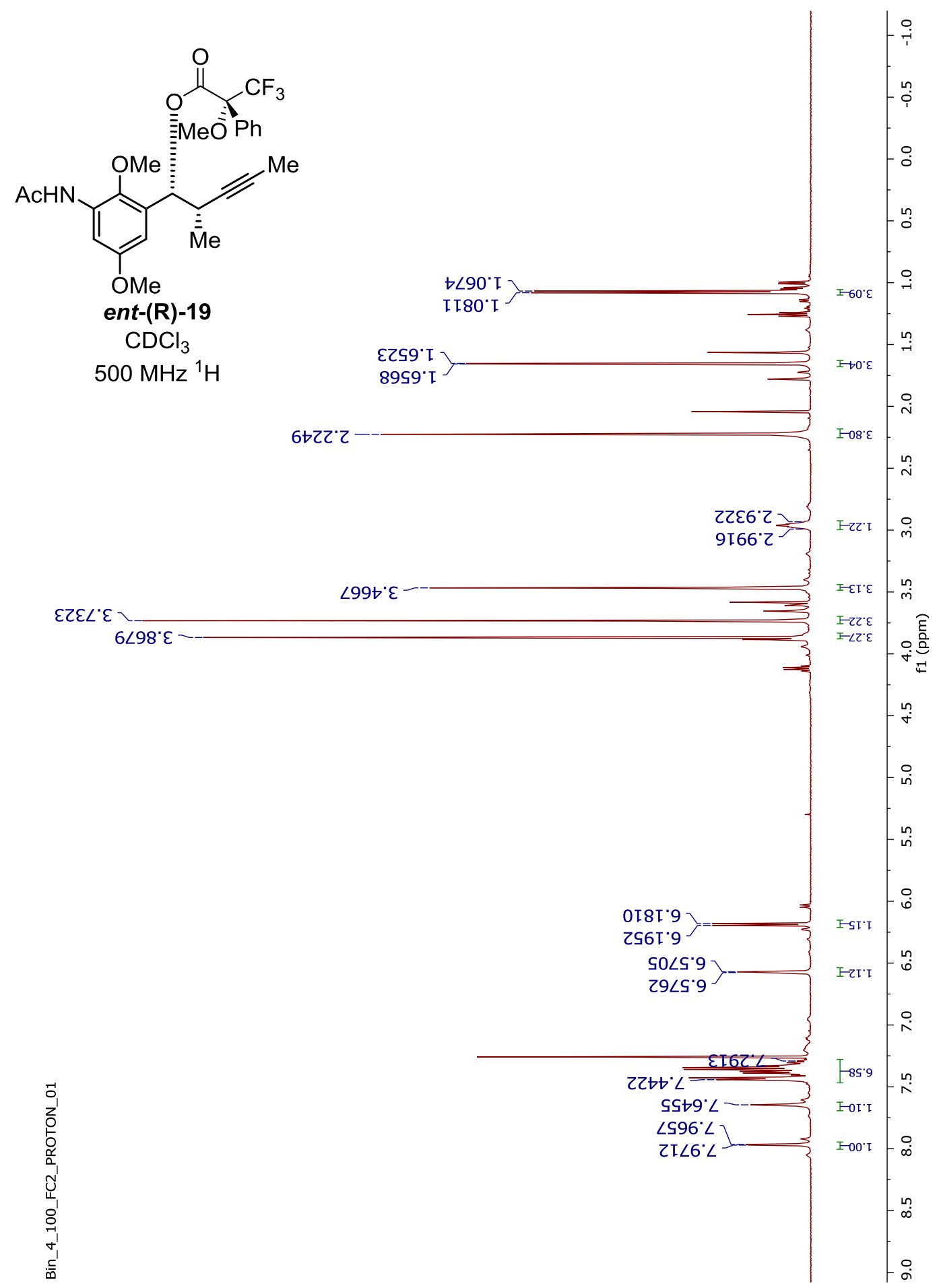

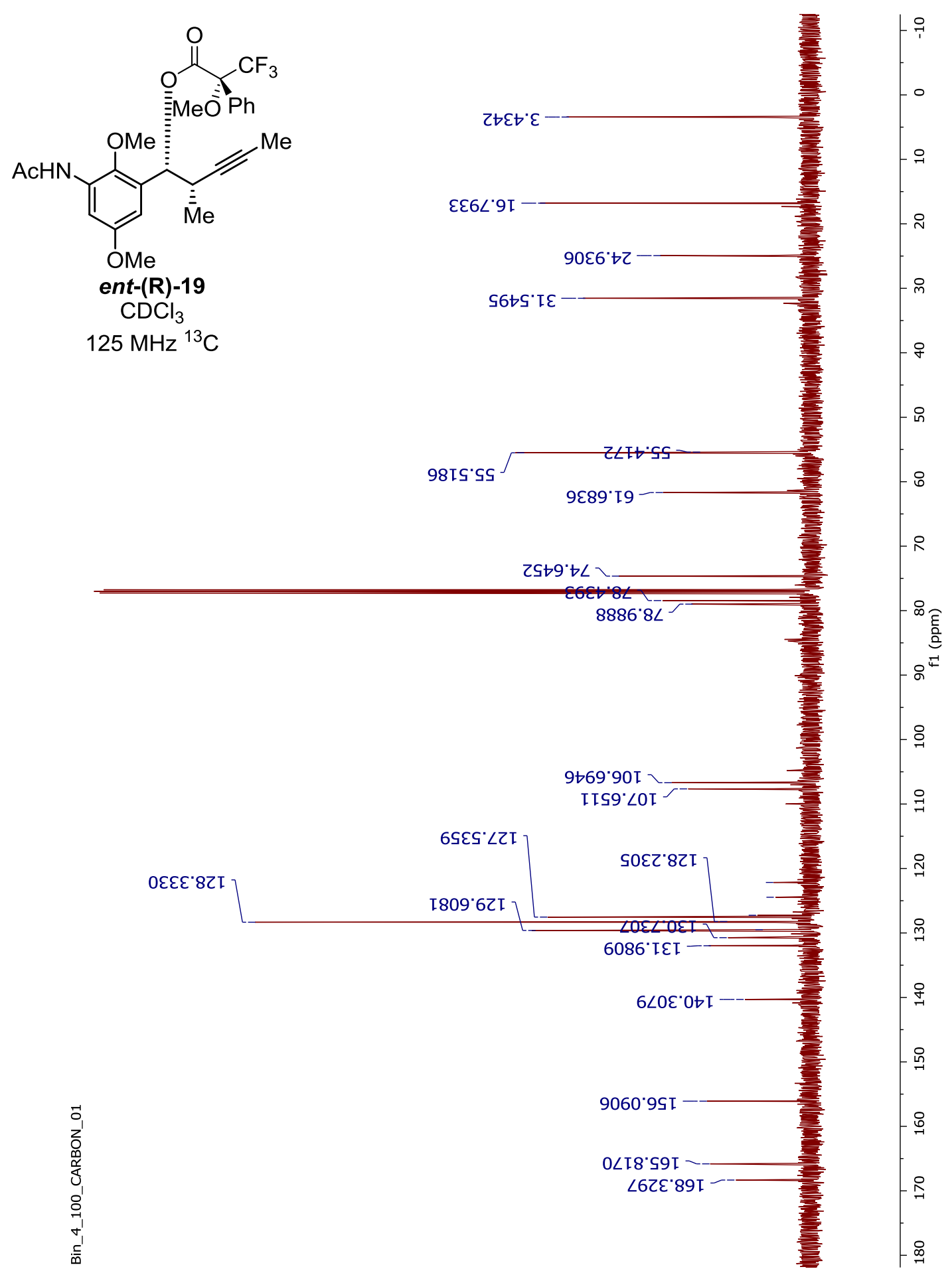

SI-110 